University of Rhode Island

\title{
DigitalCommons@URI
}

Open Access Dissertations

2017

\section{Direct Substrate Recognition by the ATP- Dependent Chaperone Protease CLPXP and Roles in Proteostasis}

Marissa Grace Viola

University of Rhode Island, marissa_viola@my.uri.edu

Follow this and additional works at: http://digitalcommons.uri.edu/oa_diss

Terms of Use

All rights reserved under copyright.

\section{Recommended Citation}

Viola, Marissa Grace, "Direct Substrate Recognition by the ATP-Dependent Chaperone Protease CLPXP and Roles in Proteostasis" (2017). Open Access Dissertations. Paper 663.

http://digitalcommons.uri.edu/oa_diss/663

This Dissertation is brought to you for free and open access by DigitalCommons@URI. It has been accepted for inclusion in Open Access Dissertations by an authorized administrator of DigitalCommons@URI. For more information, please contact digitalcommons@etal.uri.edu. 


\title{
DIRECT SUBSTRATE RECOGNITION BY THE ATP-DEPENDENT CHAPERONE PROTEASE CLPXP AND ROLES IN PROTEOSTASIS
}

\author{
BY
}

MARISSA GRACE VIOLA

\begin{abstract}
A DISSERTATION SUBMITTED IN PARTIAL FULFILLMENT OF THE
REQUIREMENTS FOR THE DEGREE OF

DOCTOR OF PHILOSOPHY

IN

CELL AND MOLECULAR BIOLOGY
\end{abstract}

UNIVERSITY OF RHODE ISLAND

2017 
DOCTOR OF PHILOSOPHY DISSERTATION

$\mathrm{OF}$

MARISSA GRACE VIOLA

APPROVED:

Dissertation Committee:

Major Professor Jodi L. Camberg

David R. Nelson

Alison Roberts

Nasser H. Zawia

DEAN OF THE GRADUATE SCHOOL

UNIVERSITY OF RHODE ISLAND

2017 


\begin{abstract}
The AAA+ (ATPases associated with a variety of cellular activities) protein superfamily includes approximately 30,000 molecular machines powered by adenosine triphosphate (ATP) binding and hydrolysis events, which are important for many cellular processes, including cell division, DNA replication, intracellular transport, and protein quality control. The bacterial AAA+ protein ClpXP is a two component ATP-dependent chaperone-protease that recognizes protein substrates bearing specific recognition signals, subsequently unfolding and degrading them to eliminate unnecessary or misfolded proteins. Since degradation is irreversible, highly specific recognition motifs are needed to ensure intentional engagement. A common strategy to facilitate recognition of substrates by AAA+ ATPases is to display multivalent recognition motifs, usually as a result of oligomerization or polymerization. During cell division in Escherichia coli, it has been reported that ClpXP degrades the essential cell division protein FtsZ (the prokaryotic tubulin homolog) in both the monomer and polymer form, however the degrons important for $\mathrm{ClpX}$ recognition of FtsZ, the mechanism by which $\mathrm{ClpX}$ recognizes and degrades FtsZ, and the physiological relevance for this regulated proteolytic event were previously unknown. We review more about substrate discrimination, multivalent recognition, and processive unfolding of FtsZ by ClpXP in Manuscript I.
\end{abstract}

In Manuscript II, we identified regions important for ClpXP targeting the native substrate FtsZ. First, we mutagenized FtsZ mutant proteins and degraded these proteins in vitro to deduce the regions (known as degrons) important for degradation by ClpXP. Then, we examined relevant Gfp-tagged FtsZ mutant proteins in dividing E. coli cells in 
vivo to further understand the importance of impairing regulated proteolysis on FtsZ assembly and cell division.

In Manuscript III, we propose the mechanism for ClpX recognition of FtsZ. We performed a traditional degradation assay with custom protein substrates in vitro to deduce the requirements for each degron for $\mathrm{ClpX}$ recognition of a monomer or polymer of FtsZ. Taken together, Manuscripts II and III describe a differential, dual-targeting role for a AAA+ substrate that will provide mechanistic insight in the field for degradation strategies in the crowded, cellular milieu.

ClpXP degrades approximately 15\% of total FtsZ per cell cycle, and therefore regulated proteolysis is the proposed role of ClpXP in cell division, however, ClpXP is not essential for this process. In Manuscript IV, we examined the physiological relevance of ClpXP proteolysis during cell division in E. coli by performing photobleaching and recovery assays on Gfp-tagged FtsZ structures and measured the half-time recovery in clp-deficient strains or wildtype cells containing Gfp-tagged FtsZ mutant proteins used for Manuscript II. For the first time, we established a phenotype for cell division when regulated proteolysis was impaired using these approaches and implicated the recognition region of FtsZ by ClpX, which is shared by other modulatory cell division proteins, in the importance of septation.

Finally, using our understanding from the work described for Manuscript II, we demonstrated in Manuscript $\mathrm{V}$ that $\mathrm{ClpXP}$ recognizes and degrades aggregated FtsZ in vitro and requires the known degrons for degradation in the aggregated state. We show that recognizing FtsZ aggregates in vivo is important since there are higher FtsZ levels in clp-deficient cells compared to wild type after heat shock. Furthermore, ClpXP 
recognizes and degrades the engineered Gfp-ssrA substrate when aggregated, and that the chaperone ClpX alone promotes the reactivation of aggregated Gfp-ssrA. In conclusion, we describe a novel role for ClpXP under stressful cellular conditions and ClpX alone in disaggregation for substrates in E. coli, which broadens our understanding of the role of ClpXP in proteostasis. 


\section{ACKNOWLEDGEMENTS}

I would like to thank my advisor, Dr. Jodi L. Camberg, for providing the foundation for a lot of this dissertation, providing me with the tools to perform this work, funding me with a research assistantship for most of my time in graduate school, and supporting my travels to conferences. I would like to thank Dr. Jodi L. Camberg, Dr. David R. Nelson, Dr. Alison Roberts, and Dr. Roberta S. King, the members of my PhD committee, for their guidance with the comprehensive exam and feedback at annual graduate seminars, and finally, with this dissertation. The feedback I received at seminars from the rest of the CMB faculty and students helped shape how I communicated my research at annual conferences, and finally the manuscripts that were published and those in progress.

Finally, I thank my family and friends for their support while I was in graduate school. My parents, Anthony J. Viola, Jr. and Dr. Filippa S. Viola, consistently helped clear my mind and helped support me financially countless times. As for my boyfriend and lab mate, Christopher J. LaBreck, I could not imagine going through this process without you. 


\section{PREFACE}

This dissertation has been prepared in the Manuscript Format according to the guidelines of the Graduate School of the University of Rhode Island. Manuscript I, "Substrate discrimination, multivalent recognition, processive unfolding and degradation of the essential cell division protein FtsZ by the AAA+ chaperone-protease ClpXP," is formatted as a review article for Biological Chemistry. Manuscript II, "Location of Dual Sites in E. coli FtsZ Important for Degradation by ClpXP; One at the C-Terminus and One in the Disordered Linker," was published in PLoS One in 2014. Manuscript III, "Tandem tags drive conformation-specific substrate processing of the cell division protein FtsZ by ClpXP in Escherichia coli," was formatted for and will be submitted to Protein Science for publication. Manuscript IV, "Proteolysis-Dependent Remodeling of the Tubulin Homolog FtsZ at the Division Septum in Escherichia coli," was published in PLoS One in 2017. Manuscript V, "The Protein Chaperone ClpX Targets Native and Non-native Aggregated Substrates for Remodeling, Disassembly, and Degradation with ClpP," was published in Frontiers in Molecular Biosciences in 2017. 
TABLE OF CONTENTS

CONTENT

PAGE

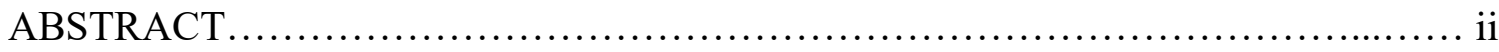

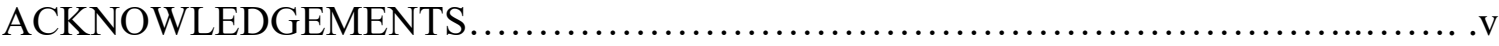

PREFACE.............................................................................

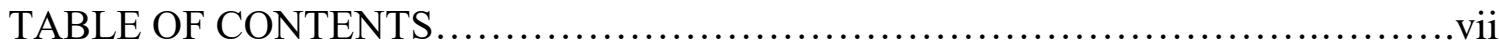

LIST OF TABLES .................................................................ii

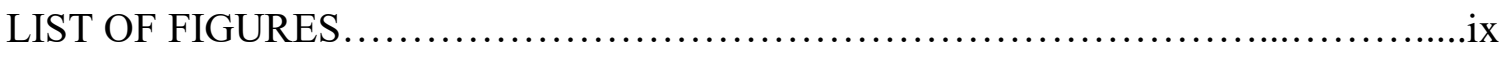

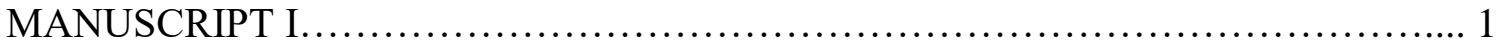

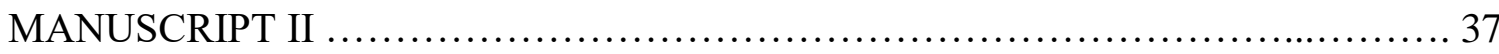

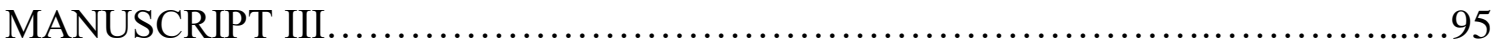

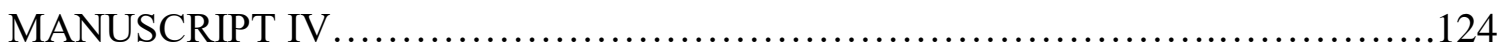

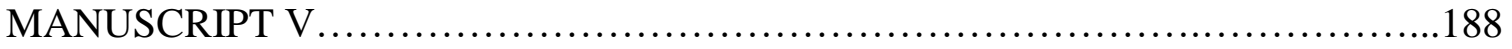




\section{LIST OF TABLES}

TABLES

PAGE

\section{MANUSCRIPT II}

Table S1. E. coli strains and plasmids used in functional assays in vivo...............83 MANUSCRIPT IV

Table 1. E. coli strains and plasmids......................................... 158

Table S1. Cell lengths and fluorescence recovery times.......................... 160 MANUSCRIPT V

Table 1. E. coli strains and plasmids used in this study...........................223 


\section{LIST OF FIGURES}

FIGURES

PAGE

MANUSCRIPT I

Figure 1. ClpXP structure from E. coli. .27

Figure 2. Z-ring assembly and FtsZ structure................................29

Figure 3. Recognition of the FtsZ C-terminus by cell division regulators...............31

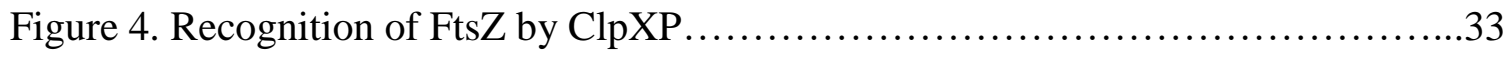

Figure 5. Impact of ClpXP degradation of FtsZ dynamics during cell division............35 MANUSCRIPT II

Figure 1. The FtsZ C-terminus is important for ClpXP degradation...

Figure 2. Residues in the linker region of FtsZ are important for degradation by ClpXP.71 Figure 3. FtsZ mutant proteins with C-terminal mutations hydrolyze GTP and assemble into polymers .73

Figure 4. A peptide corresponding to the $\mathrm{C}$-terminus of the $\mathrm{SspB}$ adaptor inhibits FtsZ

degradation. .75

Figure 5. Association of FtsZ wild type and mutant polymers with ClpX .............77

Figure 6. MinC competes with ClpXP for FtsZ in vitro...........................79

Figure 7. Mutations in the FtsZ C-terminal domain impair FtsZ function in vivo........81

Figure S1. Substitution of residues near the FtsZ C-terminus modulates the rate of

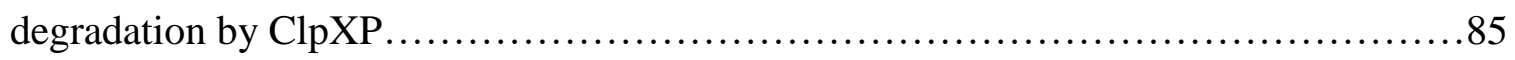

Figure S2. FtsZ mutant proteins with C-terminal mutations assemble into filament.....87

Figure S3. MinC does not inhibit degradation of GFPssrA by ClpXP................89

Figure S4. Mutations near the FtsZ C-terminal domain impair FtsZ function in vivo......91 
Figure S5. Expression of GFP-tagged FtsZ mutant proteins causes Z-ring defects ...

\section{MANUSCRIPT III}

Figure 1. Degradation of a synthetic, monomeric FtsZ substrate by ClpXP

Figure 2. ClpX engagement of monomeric and polymeric substrates....

Figure 3. Relative degron position and distance affects FtsZ degradation by

ClpXP.

Figure 4. Multimerization is important for recruitment of the FtsZ C-terminus in

vivo

Figure 5. Model for recognition of FtsZ population by ClpXP....

\section{MANUSCRIPT IV}

Figure 1. Degradation and localization of Gfp-tagged FtsZ chimeras

.161

Figure 2. Photobleaching and recovery of the Z-ring in wild type strain...............164

Figure 3. Z-ring assembly and dynamics in $c l p$ deficient strains ................... 166

Figure 4. Mutation of ClpX interaction site impairs substrate degradation in vitro and

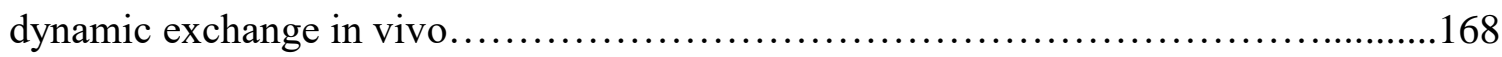

Figure 5. ZipA-Gfp ring assembly and dynamics are unaffected by deletion of $\operatorname{clpX\ldots ..170}$

Figure 6. ClpXP degradation of FtsZ polymers.............................. 172

Figure 7. Slow Z-ring fluorescence recovery half-times in strains deleted for other cell

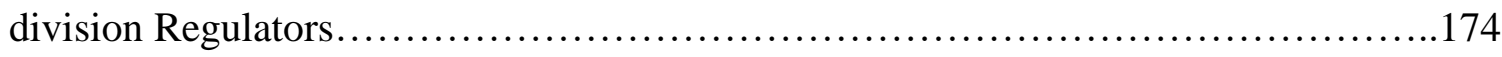

Figure S1. ClpXP degradation of Gfp and ClpX unfolding of FtsZ chimeras in vitro....176 Figure S2. Fluorescence microscopy of Z-rings and replicate recovery curves in clp

deficient strains....................................................... 178 
Figure S3. Expression of Gfp-FtsZ at various arabinose concentrations and impact on Zring Dynamics 180

Figure S4. Z-ring localization and fluorescence recovery in cells expressing Gfp-

FtsZ(R379E) or Gfp-FtsZ(G105S).

Figure S5. Degradation of FtsZ(3527A) by ClpXP in vitro and fluorescence recovery of Gfp-FtsZ(352 $7 \mathrm{~A})$ in vivo. 184

Figure S6. Fluorescence microscopy of Z-rings in cells deleted for other cell division

proteins. .186

\section{MANUSCRIPT V}

Figure 1. Disaggregation and degradation of aggregated Gfp-ssrA by ClpXP.........224

Figure 2. Aggregation and disaggregation of native ClpXP substrate FtsZ ...........227

Figure 3. Reactivation of aggregated Gfp-ssrA in the presence of ClpX..............230

Figure 4. Aggregation and disaggregation of ClpXP substrates with and without

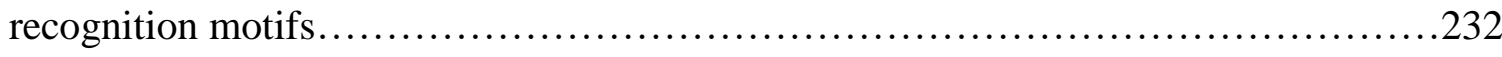

Figure 5. Disaggregation and reactivation of ClpX substrates in the presence of

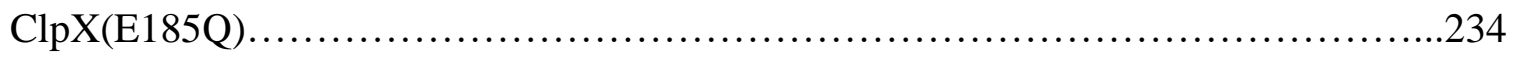

Figure 6. FtsZ aggregation in deletion strains after heat shock $\ldots \ldots \ldots \ldots \ldots \ldots \ldots \ldots . .236$

Figure 7. Model of aggregate disassembly..................................238

Figure S1. Heat-aggregation of Gfp-ssrA.................................240

Figure S2. Unfolding and degradation of aggregated Gfp-ssrA by ClpXP...........242

Figure S3. Degradation of FtsZ and FtsZ( $\Delta$ C67) by ClpXP $\ldots \ldots \ldots \ldots \ldots \ldots \ldots \ldots \ldots . \ldots \ldots \ldots$

Figure S4. Insoluble FtsZ in deletion strains after heat-treatment...................246 


\section{Manuscript I}

Publication status: formatted as a review article for Biological Chemistry

Title: Substrate discrimination, multivalent recognition, processive unfolding and degradation of the essential cell division protein FtsZ by the AAA+ chaperone-protease

$$
\text { ClpXP }
$$

Authors: Marissa G. Viola, Jodi L. Camberg ${ }^{\#}$

\section{Author Affiliations:}

Department of Cell and Molecular Biology, The University of Rhode Island, Kingston, Rhode Island, United States of America

\#Corresponding author: Jodi L. Camberg, 120 Flagg Road, Kingston, RI, 02881; Tel: (401)874-4961; Email: cambergj@uri.edu 


\section{Abstract}

All living organisms contain proteolytic enzymes that adapt to dynamic environments in the cellular milieu to catalyze post-translational, non-processive or processive degradation. $\mathrm{ClpX}$ is an ATP-dependent chaperone and well-conserved molecular machine from bacteria to humans that promotes proteostasis in both nonprocessive and processive approaches: by unfolding/remodeling and degradation of protein substrates in complex with the serine protease, ClpP. ClpX is a AAA+ ATPase and an asymmetric, homohexamer, which docks to a barrel-shaped ClpP tetradecamer. Docking opens the pore in ClpP to allow entry of unfolded polypeptides for degradation. ClpX utilizes different strategies to recognize native proteins as oligomeric complexes or polymers and targets these substrates for degradation in a regulated manner. This review summarizes the contributions of ClpXP to the essential cell division pathway in Escherichia coli in vivo, and the mechanistic insights known to date about recognition of the multivalent, polymeric substrate FtsZ by ClpX.

\section{Introduction}

In contrast to transcriptional control, proteolysis occurs post-translationally and is less energetically efficient, but allows for a rapid change in protein levels and/or dynamics as many cellular processes occur. In E. coli, there are five notable AAA+ (ATPases associated with a variety of cellular activities) which hydrolyze ATP and couple hydrolysis to unfolding and proteolysis: ClpXP, ClpAP, HslUV, Lon, and FtsH (Baker \& Sauer 2006; Sauer \& Baker 2011; Nyquist \& Martin 2014). All AAA+ proteases have a similar architecture: an oligomeric ring-like structure with a central pore, through which unfolded polypeptides are translocated, and a protease domain for degradation. The ClpX 
structure comprises a hexameric, ring-like assembly with a central pore, and docks to the tetrameric ClpP barrel (Figure 1). Degradation products are usually small peptides, ranging from 5 to 25 amino acids in length (Baker \& Sauer 2006; Sauer \& Baker 2011; Bittner et al. 2017; Siddiqui et al. 2004). ClpX is also a member of the Clp/Hsp100 family, and was recently demonstrated to disaggregate aggregated substrates (Schirmer et al. 1996; LaBreck et al. 2017). Distinct from other AAA+ ATPases, ClpX has stringent substrate specificity and can recognize substrates via specific recognition sequences or “degrons" (Sauer \& Baker 2011).

ClpX contains an N-domain, also known as the zinc-binding domain (ZBD), which dimerizes independently and is generally important for recognizing adaptor proteins and some native substrates (Figure 1) (Gottesman 2003; Wojtyra et al. 2003). Ndomain swapping studies between ClpX from E. coli and ClpX from Caulobacter crescentus showed that $\mathrm{N}$-domains from different species are interchangeable for function; however, sometimes cooperation between the $\mathrm{N}$-domain and the $\mathrm{AAA}+$ domain was impaired (Vass et al. 2017). Although the N-domain does not directly affect the interaction between the $\mathrm{AAA}+$ domain of $\mathrm{ClpX}$ and $\mathrm{ClpP}, \mathrm{ClpX}$ engagement promotes ClpP activity, and a lack of cooperation between a chimeric N-domain with a AAA+ domain may have resulted in impaired degradation of species-specific substrates (Singh et al. 2001; Vass et al. 2017). The ClpX N-domain does promote stability of the ClpX hexamer (likely due to dimerization of the $\mathrm{N}$-domains), but $\mathrm{ClpX}^{\Delta \mathrm{N}}$ was reported to bind ClpP with the same affinity as ClpX and degrade some substrates in complex with ClpP as well as ClpXP (Wojtyra et al. 2003; Singh et al. 2001; Martin et al. 2005). The Ndomain of $\mathrm{ClpX}$ has been crystallized independently from the AAA+ domain of $\mathrm{ClpX}$, 
but not in complex with substrate (Glynn et al. 2009; Donaldson et al. 2003; Kim \& Kim 2003).

$\mathrm{AAA}+$ domains have a large and a small subdomain. In ClpX, nucleotide binding occurs in the cleft between the two subdomains (large and small) of a single protomer, where the conserved Walker A, Walker B, and arginine finger sequence motifs from the large subdomain meet the sensor-II arginine sequence, a hallmark of the AAA+ protein superfamily (Neuwald et al. 1999; Erzberger \& Berger 2006). The structure of a ClpX hexamer reveals four nucleotide-bound subunits at a time (with both unbound subunits across from one another, oriented in an open conformation) (Hersch et al. 2005; Glynn et al. 2009) (Figure 1). As nucleotide is unbound, an 80-degree shift from the axial pore occurs; these conformational changes between these subdomains cause the ClpX axial pore to expand and contract, likely affecting the engagement and initial processing of substrate (Glynn et al. 2009).

Three sets of pore loops located in the upper, middle, and lower region of the ClpX channel affect polypeptide unfolding and translocation. The central loops ("pore-1" loops, GYVG sequence) are well-conserved, whereas the upper, positively-charged RKH loops enhance binding of most C-terminal motifs described for ClpX substrates (Neuwald et al. 1999; Flynn et al. 2003). The pore-2 loops, positioned at the bottom of the channel, are much larger (11 amino acids in length), and affect the overall processing of ssrAtagged substrates in combination with the RKH loops, despite the short 11-amino acid length (maximally about $20 \AA$ in length) of the ssrA tag relative to the $30 \AA$ long ClpX axial channel (Siddiqui et al. 2004; Martin et al. 2008; Martin et al. 2007). The ssrA-tag is a peptide that is appended to aborted translation products to target them for degradation 
by ClpXP. Recognition is enhanced by the adaptor protein SspB. This tagging system allows for universal recognition and clearance of these proteins (Gottesman et al. 1998; Levchenko et al. 2000). Studies demonstrating that ssrA-tagged substrates can cross-link with pore loops support a model in which RKH loops serve as specificity filters, and engagement with pore-2 loops ensures processivity of a specific substrate, perhaps due to the additional engagement of the conserved pore-1 loops which are characteristic across AAA+ proteins (Neuwald et al. 1999; Martin et al. 2008). Mutations in the speciesspecific RKH and/or pore-2 loops weaken interactions with ssrA-tagged substrates, but enhance binding of others, which suggests the structure of ClpX represents an evolutionary compromise for the recognition of a diverse class of substrates (Martin et al. 2008; Farrell et al. 2007). The conserved, hydrophobic IGF loops of ClpX dock the Cterminal surface of the ClpX hexamer to either side of the ClpP peptidase (Kim et al. 2001; Baker \& Sauer 2012).

The $c l p X$ gene is downstream of the $c l p P$ gene and in the same operon in E. coli, but can be expressed independently of ClpP due to an intercistronic promoter for $\operatorname{clpX}$ alone that may be relatively weak (Yoo et al. 1994). Cellular levels of ClpXP are higher during logarithmic growth phase in E. coli, but $c l p P$ alone is induced during stationary phase (Yoo et al. 1994). ClpXP degrades RpoS $\left(\sigma^{\mathrm{S}}\right)$, the RNA polymerase subunit that is upregulated during cell stress to activate gene expression and present during stationary phase (Schweder et al. 1996). During exponential growth, RpoS is efficiently degraded by ClpXP with the assistance of the RssB adaptor protein (Becker et al. 1999; Zhou et al. 2001). ClpX is not essential in E. coli, but neither are the other AAA+ ATPases with the exception of FtsH (Bittner et al. 2017). Regardless, ClpXP performs specific, recognition- 
mediated proteolysis of substrates for several important cellular processes under certain conditions, such as intracellular transport, DNA replication, protein quality control, and cell division (Snider et al. 2008). ClpXP degrades the essential cell division protein FtsZ, although ClpXP is not necessary for cell viability. However, modest ClpXP overexpression causes cell filamentation and perturbed cell division, suggesting that regulated proteolysis is important for this process (Camberg et al. 2009).

\section{FtsZ assembly and regulation during cell division by a network of regulators, including ClpXP}

During division, cell division proteins are spatially and temporally regulated to ensure proper placement of the Z-ring at midcell and subsequent septation (Hirota et al. 1968) (Figure 2A). The Z-ring contains bundled filaments of a highly conserved protein across bacterial species known as "FtsZ" (named for "Filamenting temperature-sensitive Z-mutant," since temperature-sensitive filamentation was the phenotype in a screen for cell division proteins) (Hirota et al. 1968) (Figure 2A). In vivo studies in dividing cells using various high-resolution microscopy techniques have shown that Z-rings in rodshaped E. coli and B. subtilis, spherical Staphylococcus aureus and Streptococcus pneumoniae, and curved/rod-shaped C. crescentus are discontinuous structures that contain a network of overlapping bundles of FtsZ polymers (Fu et al. 2010; Szwedziak et al. 2014; Strauss et al. 2012; Jacq et al. 2015; Li et al. 2007; Holden et al. 2014) (Figure $2 \mathrm{~A})$. Although only approximately $30 \%$ of the total cellular FtsZ is present in the Z-ring at any given time, the Z-ring is a highly dynamic structure that rapidly exchanges FtsZ subunits from a cytoplasmic pool and is also frequently observed as a loose, helical structure rather than a closed ring (Stricker et al. 2002; Anderson et al. 2004). 
Photobleaching and fluorescence recovery assays of cells expressing green fluorescent protein (Gfp) fused to FtsZ show that Z-ring fluorescence recovers with a half-time of approximately $9 \mathrm{sec}$ and $8 \mathrm{sec}$ in E. coli and B. subtilis respectively (Anderson et al. 2004). Given the similarity in subunit exchange rates for a gram-negative and grampositive organism, Z-ring dynamics are likely conserved across bacterial species (Stricker et al. 2002; Anderson et al. 2004).

The structural model of FtsZ shows a large, globular polymerization (GTPase) domain (residues 1-316), a flexible, unstructured linker region (residues 317-369), and a helical C-terminus as shown in co-crystal structures with other division proteins (residues 370-383) (Nogales et al. 1998; Oliva et al. 2004; Mosyak et al. 2000; Szwedziak et al. 2012) (Figure 2B). FtsZ is structurally homologous to the eukaryotic protein tubulin, but has poor sequence similarity. All FtsZ subunits are the same, but tubulin has alpha and beta subunits; however, both assemble in a head-to-tail arrangement and have been reported to exhibit treadmilling activity in vivo (Figure 2B) (Bisson-Filho et al. 2017; Wagstaff et al. 2017). Both FtsZ and tubulin assemble into linear filaments in vitro in the presence of GTP and catalyze GTP hydrolysis (Figure 2B) (Erickson et al. 1996; Erickson \& Stoffler 1996; Nogales et al. 1998). Like tubulin, FtsZ polymerizes in the presence of GTP, and upon nucleotide binding, conformational changes at the surface of the polymerization domains allow monomers to self-associate and polymerize at the inner face of the cytoplasmic membrane at midcell (Figure 2A and 2B). ClpXP degrades both non-polymerized FtsZ (which exists as monomers and dimers) and polymers in vitro (Mukherjee \& Lutkenhaus 1994; Erickson et al. 1996; Di Lallo et al. 1999; J L Camberg et al. 2009). 
During division, FtsZ is restricted to midcell by two mechanisms: direct inhibition of polymerization by the Min system, which oscillates to create a concentration gradient higher at cell poles, and nucleoid occlusion by the protein SImA, which simultaneously binds FtsZ and the nucleoid to prevent constriction over the nucleoid regions; cells deleted for $\min C$ and $\operatorname{sim} A$ are synthetic lethal (Cho et al. 2011; Tonthat et al. 2011; Bernhardt \& De Boer 2005). Once FtsZ polymers are restricted to midcell, the membrane tethering proteins ZipA and FtsA anchor FtsZ polymers to the inner face of the cytoplasmic membrane (Pichoff \& Lutkenhaus 2002). Stabilizing proteins laterally and longitudinally bundle FtsZ filaments to counterbalance destabilizing proteins before constriction, likely to promote loose, accessible FtsZ subunits to coordinate constriction during late septal phase (Huang et al. 2013). ClpXP degrades 15\% of total FtsZ per cell cycle and is the only cell division regulator among approximately 20 proteins that degrades FtsZ subunits processively to destabilize FtsZ polymers (Camberg et al. 2009). The destabilizing proteins $\mathrm{ClpXP}$, MinC, and SlmA bind FtsZ via the C-terminus at distinct regions, but substrate hand-off may occur to regulate Z-ring assembly and disassembly since ClpXP competes with MinC for FtsZ in vitro (Camberg et al. 2014) (Figure 3). The tethering proteins ZipA and FtsA also interact with FtsZ via the Cterminus at adjacent regions (Figure 3).

\section{ClpXP recognizes and degrades FtsZ via two distinct regions during division}

FtsZ is an N-domain dependent substrate of ClpXP and is recognized and degraded via two distinct recognition motifs at the $\mathrm{C}$-terminus that resemble the $\mathrm{C}$-motif 2 degron characterized for ClpX C-terminal substrates (Figure 4A) (Flynn et al. 2003; Camberg et al. 2014). The FtsZ recognition sequences, or "degrons," are structurally 
distinct (one is in the center of an intrinsically disordered region, and one at the extreme C-terminal alpha helix), but resemble the same C-motif 2 consensus sequence as described for C-terminal degrons recognized by ClpX (Figure 4A) (Gardner et al. 2013; Flynn et al. 2003). ClpX recognition of the C-terminal degron is consistent with engagement of other ClpX substrates (which is generally N-or-C-terminal) and other cell division regulators, which modulate FtsZ via the extreme C-terminus in overlapping regions (Figure 3).

The linker degron is uncharacteristic not only due to the lack of structure in this region, but also because no other proteins are known to interact with FtsZ in this region (Gardner et al. 2013). Unlike the polymerization domain and C-terminal regions, the linker region is the least conserved across bacterial species and can range in length from 9 to over 300 amino acids. The long linker in FtsZ from C. crescentus has been implicated in additional functions for peptidoglycan remodeling during late septal phases of division (Sundararajan et al. 2015). In Bacillus subtilis, as long as the sequence was intrinsically disordered, the specific amino acids did not matter, but the original length was required for proper division in vivo and FtsZ assembly in vitro (Buske \& Levin 2012). The linker region is proposed to act as a flexible tether for the $\mathrm{C}$-terminus to promote engagement of FtsZ regulatory proteins, possibly due to the species-specific differences in FtsZ polymerization properties and dynamics. Similarly, the extreme Cterminus of FtsZ is hypervariable, and likely allows species-specific cell division regulators to recognize FtsZ (Buske \& Levin 2013).

The rate of degradation is $2-3$ fold faster for FtsZ polymers than for FtsZ monomers (Camberg et al. 2009). Since an FtsZ monomer contains two degrons, FtsZ 
monomers alone are multivalent substrates, and ClpX may utilize presentation of the two degrons to differentiate between monomer and polymer formation, which could explain the differences in degradation rates. ClpXP also degrades another native oligomeric substrate, Dps, which is a dodecameric protein complex that protects the nucleoid from DNA damage during stationary phase (Stephani et al. 2003). Like FtsZ, ClpXP degrades the phage protein MuA as a tetramer more efficiently than individual subunits, leading to destabilization of the MuA transposition complex (Abdelhakim et al. 2010; Abdelhakim et al. 2008; Ling et al. 2015). In the model for FtsZ degradation, ClpXP promotes destabilization of FtsZ polymers in two ways: (1) monomers are degraded, which shifts the dynamic equilibrium of FtsZ polymers towards disassembly and (2) ClpXP degrades FtsZ polymers directly, leading to polymer severing (Figure 4B and 4C).

\section{Physiological relevance for ClpXP degradation of FtsZ}

Of all known FtsZ regulators, ClpX is one of the most highly conserved across all bacterial species. ClpX has been shown to prevent and modulate FtsZ assembly in E. coli, B. subtilis, and M. tuberculosis in vitro, but only proteolysis by ClpXP is important for FtsZ turnover in E. coli and C. crescentus (Camberg et al. 2009; Weart et al. 2005; Haeusser et al. 2009; Sugimoto et al. 2010; Dziedzic et al. 2010; Williams et al. 2014). A proteomic screen in S. aureus identified FtsZ as a substrate for ClpP degradation (Feng et al. 2013).

In E. coli, deletion of $c l p X$ or $c l p P$ does not cause cell filamentation or Z-ring misplacement (Camberg et al. 2009; Viola et al. 2017). Since FtsZ is an essential protein for an essential process, why is regulating turnover not required for cell division in vivo? Deletion of $\min C$ causes a visible cell division phenotype consisting of minicells, 
multiple, misplaced Z-rings and cells up to 3-times longer than wild type cells (Yu \& Margolin 1999; Teather et al. 1974). Gfp-FtsZ subunit turnover in fluorescent Z-rings of dividing cells was measured in photobleaching and recovery experiments in cells deleted for $\operatorname{clpX}$, $\operatorname{clp} P$, and cells containing the proteolysis mutant protein $\operatorname{clp} P(S 97 A)$ in place of $c l p P$ on the chromosome revealed a $70 \%$ longer half-time recovery than for FtsZ subunits in wild type Z-rings (Viola et al. 2017). These results implicated ClpXP as physiologically relevant for FtsZ subunit turnover in a proteolysis-dependent manner. Comparably, cells deleted for $\operatorname{sim} A$ had an $80 \%$ slower recovery rate than wild type, and cells deleted for $\min C$ were $70 \%$ slower, and emphasized the global role of destabilizing FtsZ and the impact on Z-ring dynamics in vivo (Viola et al. 2017). Furthermore, impairing a conserved residue in FtsZ (R379), which is important for both ClpX and FtsA recognition (Figure 3), dramatically perturbed Z-ring subunit turnover by 2-fold (Viola et al. 2017; Szwedziak et al. 2012). Therefore, ClpXP promotes the dynamic exchange of FtsZ subunits in the Z-ring in live, dividing cells by proteolysis.

\section{ClpXP degrades FtsZ aggregates}

ClpX is a member of the Hsp100/Clp family of proteins and was recently shown to be capable of disassembling aggregated substrates, including Gfp-ssrA and FtsZ (Schirmer et al. 1996; LaBreck et al. 2017). ClpX associates with cellular aggregates in E. coli, and both $\mathrm{ClpX}$ and $\mathrm{ClpP}$ colocalized with cellular inclusion bodies in B. subtilis under heat stress (Kain et al. 2008; Simmons et al. 2008; Vera et al. 2005; Winkler et al. 2010; Kirstein et al. 2008; Maisonneuve et al. 2008; Krüger et al. 2014). In vitro, ClpX functions as a traditional chaperone to protect the lambda $\mathrm{O}$ phage protein from aggregation and promote its refolding (Wawrzynow et al. 1995). ClpX was recently 
shown to degrade Gfp-ssrA and FtsZ aggregates in vitro, and degrade FtsZ aggregates in vivo (LaBreck et al. 2017).

ClpX expression does not significantly increase when cells experience heat shock, but FtsZ aggregates accumulate significantly in cells deleted for $\operatorname{clp} X$ or $c l p P$, but not other chaperones, suggesting that ClpXP may remove aggregates with specific degrons in vivo (LaBreck et al. 2017). In vitro, neither untagged Gfp aggregates nor FtsZ aggregates lacking the $\mathrm{C}$-terminus (FtsZ( $\Delta \mathrm{C} 67)$ aggregates) are degraded by $\mathrm{ClpXP}$, which suggests that degrons are required for aggregate recognition and removal (LaBreck et al. 2017). Although ClpX alone binds to and promotes refolding of Gfp-ssrA, in E. coli, ClpX likely exists in complex with ClpP and may function in aggregate clearance in vivo (Li et al. 2000; LaBreck et al. 2017).

\section{Concluding remarks}

ClpXP degrades approximately $15 \%$ of total FtsZ in actively dividing cells and promotes the dynamic exchange of subunits in the Z-ring in E. coli (Camberg et al. 2009; Viola et al. 2017). The multivalent targeting strategy ClpX utilizes contributes to robust discrimination of substrates in the crowded cellular milieu, and protects against irreversible degradation of other cellular proteins (Baker \& Sauer 2006). The increased affinity of ClpXP for subunits within a polymer, which leads to polymer breakage, would favor accessibility of polymer ends and may promote faster polymer remodeling, and therefore the dynamic exchange of subunits (Figure 5A). This is consistent with the idea that the Z-ring comprises a loose network of FtsZ polymers based on high-resolution microscopy of Z-rings in several bacterial species, and places ClpXP in a role that favors this cytokinetic structure (Figure 5B). Other cell division regulators, such as SlmA and 
ZipA, also utilize a multivalent recognition strategy for FtsZ and have a stronger affinity for polymerized FtsZ in the cytokinetic septal ring (Du et al. 2015). Cell division proteins MinD and FtsA both contain ClpX degrons and were identified as potential substrates for ClpXP under cell stress conditions; there is evidence that MinD, FtsA, and FtsZ polymerize (copolymers of MinD with MinC, and FtsA in complex with FtsZ along lipid membranes) (Neher et al. 2006; Conti et al. 2015; Szwedziak et al. 2012; Krupka et al. 2014; Lara et al. 2005). Furthermore, cells deleted for $\min C$ and $c l p X$ have a synthetic filamentous phenotype and are longer than cells deleted for $\min C$ alone (Camberg et al. 2011). Therefore, the physiological role of ClpXP in cell division may be more diverse than previously thought.

Since ClpXP degrades Gfp-ssrA aggregates in vitro and FtsZ aggregates in vitro and potentially in vivo, the role for ClpXP in protein quality control with respect to universal substrates and native substrates may also be broader than previously determined (LaBreck et al. 2017). Prior studies on ClpX disaggregase activity focused on non-native substrates or association with inclusion bodies, and did not directly implicate ClpXP in this physiological role in vivo for known substrates in E. coli until now. The ability for ClpXP to recognize and degrade aggregates in vivo would relieve the other heat shock proteins, since ClpXP recognizes over 100 diverse cellular substrates, and would promote proteostasis (Flynn et al. 2003; Neher et al. 2006). Polymers, by definition, are ordered aggregates, and therefore $\mathrm{ClpX}$ substrates that aggregate would be targeted for $\mathrm{ClpX}$ recognition under stressful cellular conditions. Since the capacity for ClpX disaggregation has been demonstrated, further proteomic studies are necessary to determine the full contribution of ClpXP aggregate clearance to survival under stress 
conditions.

\section{References}

Abdelhakim, A.H. et al., 2008. Unique Contacts Direct High-Priority Recognition of the Tetrameric $\mathrm{Mu}$ Transposase-DNA Complex by the AAA+ Unfoldase ClpX. Molecular Cell, 30(1), pp.39-50.

Abdelhakim, A.H., Sauer, R.T. \& Baker, T. a, 2010. The AAA+ ClpX machine unfolds a keystone subunit to remodel the $\mathrm{Mu}$ transpososome. Proceedings of the National Academy of Sciences of the United States of America, 107(6), pp.2437-2442.

Anderson, D.E., Gueiros-Filho, F.J. \& Erickson, H.P., 2004. Assembly dynamics of FtsZ rings in Bacillus subtilis and Escherichia coli and effects of FtsZ-regulating proteins. Journal of Bacteriology, 186(17), pp.5775-5781.

Baker, T.A. \& Sauer, R.T., 2012. ClpXP, an ATP-powered unfolding and proteindegradation machine. Biochimica et Biophysica Acta - Molecular Cell Research, 1823(1), pp.15-28. Available at: http://dx.doi.org/10.1016/j.bbamcr.2011.06.007.

Baker, T. a. \& Sauer, R.T., 2006. ATP-dependent proteases of bacteria: recognition logic and operating principles. Trends in Biochemical Sciences, 31(12), pp.647-653. Available at: http://linkinghub.elsevier.com/retrieve/pii/S0968000406002969.

Becker, G., Klauck, E. \& Regine, H.-A., 1999. Regulation of RpoS proteolysis in Escherichia coli: The response regulator $\mathrm{RssB}$ is a recognition factor that interacts with the turnover element in RpoS. , 96(May), pp.6439-6444.

Bernhardt, T.G. \& De Boer, P. a J., 2005. SlmA, a nucleoid-associated, FtsZ binding protein required for blocking septal ring assembly over chromosomes in E. coli. 
Molecular Cell, 18(5), pp.555-564.

Bisson-Filho, A.W. et al., 2017. Treadmilling by FtsZ filaments drives peptidoglycan synthesis and bacterial cell division. Science, 355(6326), pp.739-743. Available at: http://www.sciencemag.org/lookup/doi/10.1126/science.aak9973.

Bittner, L.M., Arends, J. \& Narberhaus, F., 2017. When, how and why? Regulated proteolysis by the essential FtsH protease in Escherichia coli. Biological Chemistry, 398(5-6), pp.625-635.

Buske, P.J. \& Levin, P.A., 2012. Extreme C terminus of bacterial cytoskeletal protein FtsZ plays fundamental role in assembly independent of modulatory proteins. Journal of Biological Chemistry, 287(14), pp.10945-10957.

Buske, P.J. \& Levin, P. a, 2013. The C Terminus of FtsZ Regulates FtsZ Assembly Dynamics and Is Required for Bacillus Subtilis Cell Division.

Camberg, J.L. et al., 2014. Location of dual sites in E. coli FtsZ important for degradation by ClpXP; one at the C-terminus and one in the disordered linker. PLoS ONE, 9(4).

Camberg, J.L., Hoskins, J.R. \& Wickner, S., 2009. ClpXP protease degrades the cytoskeletal protein, FtsZ, and modulates FtsZ polymer dynamics. Proc Natl Acad Sci $U \quad S \quad A, \quad$ 106(26), pp.10614-10619. Available at: http://www.ncbi.nlm.nih.gov/pubmed/19541655.

Camberg, J.L., Hoskins, J.R. \& Wickner, S., 2009. ClpXP protease degrades the cytoskeletal protein, FtsZ, and modulates FtsZ polymer dynamics. Proceedings of 
the National Academy of Sciences of the United States of America, 106(26), pp.10614-10619.

Camberg, J.L., Hoskins, J.R. \& Wickner, S., 2011. The Interplay of ClpXP with the cell division machinery in Escherichia coli. Journal of Bacteriology, 193(8), pp.19111918.

Cho, H. et al., 2011. Nucleoid occlusion factor SlmA is a DNA-activated FtsZ polymerization antagonist. Proceedings of the National Academy of Sciences, 108(9), pp.3773-3778. Available at: http://www.pnas.org/cgi/doi/10.1073/pnas.1018674108.

Conti, J., Viola, M.G. \& Camberg, J.L., 2015. The bacterial cell division regulators MinD and MinC form polymers in the presence of nucleotide. FEBS Letters, 589(2), pp.201-206. Available

at: http://inkinghub.elsevier.com/retrieve/pii/S0014579314008631.

Donaldson, L.W., Wojtyra, U. \& Houry, W.A., 2003. Solution structure of the dimeric zinc binding domain of the chaperone ClpX. The Journal of biological chemistry, 278(49), pp.48991-48996.

Du, S., Park, K.-T. \& Lutkenhaus, J., 2015. Oligomerization of FtsZ converts the FtsZ tail motif (conserved carboxy-terminal peptide) into a multivalent ligand with high avidity for partners ZipA and SlmA. Molecular Microbiology, 95(2), pp.173-188. Available at: http://doi.wiley.com/10.1111/mmi.12854.

Dziedzic, R. et al., 2010. Mycobacterium tuberculosis ClpX interacts with FtsZ and interferes with FtsZ assembly. PloS one, 5(7), p.e11058. Available at: 
http://www.pubmedcentral.nih.gov/articlerender.fcgi?artid=2897852\&tool=pmcen trez\&rendertype $=$ abstract.

Erickson, H.P. et al., 1996. Bacterial cell division protein FtsZ assembles into protofilament sheets and minirings, structural homologs of tubulin polymers. Proceedings of the National Academy of Sciences of the United States of America, 93(1), pp.519-523.

Erickson, H.P. \& Stoffler, D., 1996. Protofilaments and rings, two conformations of the tubulin family conserved from bacterial FtsZ to alpha/beta and gamma tubulin. The Journal of cell biology, 135(1), pp.5-8.

Erzberger, J.P. \& Berger, J.M., 2006. EVOLUTIONARY RELATIONSHIPS AND STRUCTURAL MECHANISMS OF AAA+ PROTEINS. Annual Review of Biophysics and Biomolecular Structure, 35(1), pp.93-114. Available at: https://doi.org/10.1146/annurev.biophys.35.040405.101933.

Farrell, C.M., Baker, T.A. \& Sauer, R.T., 2007. Altered Specificity of a AAA+ Protease. Molecular Cell, 25(1), pp.161-166.

Feng, J. et al., 2013. Trapping and proteomic identification of cellular substrates of the ClpP protease in staphylococcus aureus. Journal of Proteome Research, 12, pp.547-558.

Flynn, J.M. et al., 2003. Proteomic discovery of cellular substrates of the ClpXP protease reveals five classes of ClpX-recognition signals. Molecular Cell, 11(3), pp.671683. 
Fu, G. et al., 2010. In Vivo structure of the E. coli FtsZ-ring revealed by photoactivated localization microscopy (PALM). PLoS ONE, 5(9), pp.1-16.

Gardner, K. a J.A., Moore, D. a. \& Erickson, H.P., 2013. The C-terminal linker of Escherichia coliFtsZ functions as an intrinsically disordered peptide. Molecular Microbiology, 89(2), pp.264-275.

Glynn, S.E. et al., 2009. Structures of Asymmetric ClpX Hexamers Reveal NucleotideDependent Motions in a AAA+ Protein-Unfolding Machine. Cell, 139(4), pp.744756. Available at: http://dx.doi.org/10.1016/j.cell.2009.09.034.

Gottesman, S., 2003. Proteolysis in Bacterial Regulatory Circuits. Annual Review of Cell and Developmental Biology, 19(1), pp.565-587. Available at: http://www.annualreviews.org/doi/10.1146/annurev.cellbio.19.110701.153228.

Gottesman, S. et al., 1998. The ClpXP and ClpAP proteases degrade proteins with carboxy-terminal peptide tails added by the SsrA-tagging system. Genes and Development, 12(9), pp.1338-1347.

Haeusser, D.P. et al., 2009. ClpX Inhibits FtsZ Assembly in a manner that does not require its ATP hydrolysis-dependent chaperone activity. Journal of Bacteriology, 191(6), pp.1986-1991.

Hersch, G.L. et al., 2005. Asymmetric interactions of ATP with the AAA+ ClpX6 unfoldase: Allosteric control of a protein machine. Cell, 121(7), pp.1017-1027.

Hirota, Y. et al., 1968. On the process of cellular division in Escherichia coli. Journal of Molecular Biology, 35(1), pp.175-192. Available at: 
http://www.sciencedirect.com/science/article/pii/S0022283668800464 [Accessed February 9, 2016].

Holden, S.J. et al., 2014. High throughput 3D super-resolution microscopy reveals Caulobacter crescentus in vivo Z-ring organization. Proceedings of the National Academy of Sciences, 111(12), pp.4566-4571. Available at: http://www.pnas.org/cgi/doi/10.1073/pnas.1313368111.

Huang, K.H., Durand-Heredia, J. \& Janakiraman, A., 2013. FtsZ ring stability: Of bundles, tubules, crosslinks, and curves. Journal of Bacteriology, 195(9), pp.1859-1868.

Jacq, M. et al., 2015. Remodeling of the Z-ring Nanostructure during the Streptococcus pneumoniae Cell Cycle Revealed by Photoactivated Localization Microscopy. mBio, 6(4).

Jenal, U. \& Fuchs, T., 1998. An essential protease involved in bacterial cell-cycle control. , 17(19), pp.5658-5669.

Kain, J., He, G.G. \& Losick, R., 2008. Polar localization and compartmentalization of ClpP proteases during growth and sporulation in Bacillus subtilis. Journal of Bacteriology, 190(20), pp.6749-6757.

Kim, D.Y. \& Kim, K.K., 2003. Crystal Structure of ClpX Molecular Chaperone from Helicobacter pylori. Journal of Biological Chemistry, 278(50), pp.50664-50670.

Kim, Y.-I. et al., 2001. Molecular determinants of complex formation between Clp/Hsp100 ATPases and the ClpP peptidase. Nat Struct Mol Biol, 8(3), pp.230- 
233. Available at: http://dx.doi.org/10.1038/84967.

Kirstein, J. et al., 2008. Localization of general and regulatory proteolysis in Bacillus subtilis cells. Molecular Microbiology, 70(3), pp.682-694.

Krüger, E. et al., 2014. The Clp Proteases of Bacillus subtilis Are Directly Involved in Degradation of Misfolded Proteins The Clp Proteases of Bacillus subtilis Are Directly Involved in Degradation of Misfolded Proteins. , 182(11), pp.3259-3265.

Krupka, M. et al., 2014. Role of the FtsA C terminus as a switch for polymerization and membrane association. mBio, 5(6), pp.1-9.

LaBreck, C.J. et al., 2017. The Protein Chaperone ClpX Targets Native and Non-native Aggregated Substrates for Remodeling, Disassembly, and Degradation with ClpP. Frontiers in Molecular Biosciences, 4(May), pp.1-13. Available at: http://journal.frontiersin.org/article/10.3389/fmolb.2017.00026/full.

Di Lallo, G. et al., 1999. FtsZ dimerization in vivo. Molecular microbiology, 32(2), pp.265-74. Available at: http://www.ncbi.nlm.nih.gov/pubmed/10231484.

Lara, B. et al., 2005. Cell division in cocci: Localization and properties of the Streptococcus pneumoniae FtsA protein. Molecular Microbiology, 55(3), pp.699711.

Levchenko, I. et al., 2000. A Specificity-Enhancing Factor for the ClpXP Degradation Machine. Science, 289(5488), p.2354 LP-2356. Available at: http://science.sciencemag.org/content/289/5488/2354.abstract.

Li, C., Yi Ping Tao \& Simon, L.D., 2000. Expression of different-size transcripts from the 
clpP-clpX operon of Escherichia coli during carbon deprivation. Journal of Bacteriology, 182(23), pp.6630-6637.

Li, Z. et al., 2007. The structure of FtsZ filaments in vivo suggests a force-generating role in cell division. The EMBO Journal, 26(22), pp.4694-4708. Available at: http://emboj.embopress.org/cgi/doi/10.1038/sj.emboj.7601895.

Ling, L. et al., 2015. Deciphering the roles of the multi-component recognition signals by the AAA+ unfoldase, ClpX. Journal of Molecular Biology, 427(18), pp.29662982.

Maisonneuve, E. et al., 2008. Existence of abnormal protein aggregates in healthy Escherichia coli cells. Journal of Bacteriology, 190(3), pp.887-893.

Martin, A., Baker, T.A. \& Sauer, R.T., 2007. Distinct Static and Dynamic Interactions Control ATPase-Peptidase Communication in a AAA+ Protease. Molecular Cell, 27(1), pp.41-52.

Martin, A., Baker, T.A. \& Sauer, R.T., 2008. Pore loops of the AAA+ ClpX machine grip substrates to drive translocation and unfolding. Nat Struct Mol Biol, 15(11), pp.1147-1151. Available at: http://dx.doi.org/10.1038/nsmb.1503.

Martin, A., Baker, T.A. \& Sauer, R.T., 2005. Rebuilt AAA + motors reveal operating principles for ATP-fuelled machines. Nature, 437(7062), pp.1115-1120. Available at: http://dx.doi.org/10.1038/nature04031.

Mosyak, L. et al., 2000. The bacterial cell-division protein ZipA and its interaction with an FtsZ fragment revealed by X-ray crystallography. The EMBO journal, 19(13), 
pp.3179-3191.

Mukherjee, a. \& Lutkenhaus, J., 1994. Guanine nucleotide-dependent assembly of FtsZ into filaments. Journal of Bacteriology, 176(9), pp.2754-2758.

Neher, S.B. et al., 2006. Proteomic Profiling of ClpXP Substrates after DNA Damage Reveals Extensive Instability within SOS Regulon. Molecular Cell, 22(2), pp.193-204.

Neuwald, A.F. et al., 1999. AAA + : A Class of Chaperone-Like ATPases Associated with the Assembly, Operation, and Disassembly of Protein Complexes. , (516), pp.2743. Available at: http://genome.cshlp.org/content/9/1/27.full.pdf.

Nogales, E. et al., 1998. Tubulin and FtsZ form a distinct family of GTPases. Nature Structural Biology, 5(6), pp.451-458.

Nyquist, K. \& Martin, A., 2014. Marching to the beat of the ring: polypeptide translocation by AAA+ proteases. Trends in Biochemical Sciences, 39(2), pp.5360. Available at: http://www.sciencedirect.com/science/article/pii/S0968000413001850.

Oliva, M.A., Cordell, S.C. \& Lowe, J., 2004. Structural insights into FtsZ protofilament formation. Nat Struct Mol Biol, 11(12), pp.1243-1250. Available at: http://dx.doi.org/10.1038/nsmb855.

Pichoff, S. \& Lutkenhaus, J., 2002. Unique and overlapping roles for ZipA and FtsA in septal ring assembly in Escherichia coli. EMBO Journal, 21(4), pp.685-693.

Sauer, R.T. \& Baker, T. a, 2011. AAA+ proteases: ATP-fueled machines of protein 
destruction. Annual review of biochemistry, 80, pp.587-612.

Schirmer, E.C. et al., 1996. HSP100/Clp proteins: a common mechanism explains diverse functions. Trends in Biochemical Sciences, 21(8), pp.289-296. Available at: http://www.sciencedirect.com/science/article/pii/S0968000496100384.

Schweder, T. et al., 1996. Regulation of lemph $\{$ Escherichia coli\} starvation sigma factor (Sigma S) by ClpZP protease. Journal of Bacteriology, 178(2), pp.470-476.

Siddiqui, S.M., Sauer, R.T. \& Baker, T. a, 2004. Role of the processing pore of the ClpX AAA + ATPase in the recognition and engagement of specific protein substrates. Genes \& Development, pp.369-374.

Simmons, L. a., Grossman, A.D. \& Walker, G.C., 2008. Clp and lon proteases occupy distinct subcellular positions in Bacillus subtilis. Journal of Bacteriology, 190(20), pp.6758-6768.

Singh, S.K. et al., 2001. Functional Domains of the ClpA and ClpX Molecular Chaperones Identified by Limited Proteolysis and Deletion Analysis. Journal of Biological Chemistry, 276(31), pp.29420-29429.

Snider, J., Thibault, G. \& Houry, W. a, 2008. The AAA+ superfamily of functionally diverse proteins. Genome biology, 9(4), p.216.

Stephani, K., Weichart, D. \& Hengge, R., 2003. Dynamic control of Dps protein levels by ClpXP and ClpAP proteases in Escherichia coli. Molecular Microbiology, 49(6), pp.1605-1614.

Strauss, M.P. et al., 2012. 3D-SIM Super Resolution Microscopy Reveals a Bead-Like 
Arrangement for FtsZ and the Division Machinery: Implications for Triggering Cytokinesis. PLoS Biology, 10(9), p.e1001389. Available at: http://dx.plos.org/10.1371/journal.pbio.1001389.

Stricker, J. et al., 2002. Rapid assembly dynamics of the Escherichia coli FtsZ-ring demonstrated by fluorescence recovery after photobleaching. Proceedings of the National Academy of Sciences of the United States of America, 99(5), pp.31713175 .

Sugimoto, S. et al., 2010. AAA+ chaperone ClpX regulates dynamics of prokaryotic cytoskeletal protein FtsZ. Journal of Biological Chemistry, 285(9), pp.66486657.

Sundararajan, K. et al., 2015. The bacterial tubulin FtsZ requires its intrinsically disordered linker to direct robust cell wall construction. Nature communications, 6, p.7281. Available at: http://www.ncbi.nlm.nih.gov/pubmed/26099469.

Szwedziak, P. et al., 2014. Architecture of the ring formed by the tubulin homologue FtsZ in bacterial cell division. eLife, 3, pp.1-22. Available at: http://elifesciences.org/lookup/doi/10.7554/eLife.04601.

Szwedziak, P. et al., 2012. FtsA forms actin-like protofilaments. The EMBO journal, $31(10)$ pp.2249-2260. Available at: http://eutils.ncbi.nlm.nih.gov/entrez/eutils/elink.fcgi?dbfrom=pubmed\&id=22473 211\&retmode=ref\&cmd=prlinks\%5Cnpapers3://publication/doi/10.1038/emboj.2 012.76 .

Teather, R.M., Collins, J.F. \& Donachie, W.D., 1974. Quantal behavior of a diffusible 
factor which initiates septum formation at potential division sites in Escherichia coli. Journal of Bacteriology, 118(2), pp.407-413.

Tonthat, N.K. et al., 2011. Molecular mechanism by which the nucleoid occlusion factor, SlmA, keeps cytokinesis in check. The EMBO journal, 30(1), pp.154-164. Available at: http://dx.doi.org/10.1038/emboj.2010.288.

Vass, R.H., Nascembeni, J. \& Chien, P., 2017. The Essential Role of ClpXP in Caulobacter crescentus Requires Species Constrained Substrate Specificity. Frontiers in Molecular Biosciences, 4(May), pp.1-9. Available at: http://journal.frontiersin.org/article/10.3389/fmolb.2017.00028/full.

Vera, A. et al., 2005. Lon and ClpP proteases participate in the physiological disintegration of bacterial inclusion bodies. Journal of Biotechnology, 119(2), pp.163-171.

Viola, M.G. et al., 2017. Proteolysis-dependent remodeling of the tubulin homolog FtsZ at the division septum in Escherichia coli. PLoS ONE, 12(1), pp.1-23.

Wagstaff, J.M. et al., 2017. crossm A Polymerization-Associated Structural Switch in FtsZ That Enables Treadmilling of Model Filaments. , 8(3), pp.1-16.

Wawrzynow, A. et al., 1995. The ClpX heat-shock protein of Escherichia coli, the ATPdependent substrate specificity component of the ClpP-ClpX protease, is a novel molecular chaperone. The EMBO Journal, 14(9), pp.1867-1877.

Weart, R.B. et al., 2005. The ClpX chaperone modulates assembly of the tubulin-like protein FtsZ. Molecular Microbiology, 57(1), pp.238-249. 
Williams, B. et al., 2014. ClpXP and ClpAP proteolytic activity on divisome substrates is differentially regulated following the Caulobacter asymmetric cell division. Molecular Microbiology, 93(August), pp.853-866.

Winkler, J. et al., 2010. Quantitative and spatio-temporal features of protein aggregation in Escherichia coli and consequences on protein quality control and cellular ageing. The EMBO journal, 29(5), pp.910-923.

Wojtyra, U.A. et al., 2003. The N-terminal zinc binding domain of ClpX is a dimerization domain that modulates the chaperone function. The Journal of biological chemistry, 278(49), pp.48981-48990.

Yoo, S.J. et al., 1994. clpX Encoding an Alternative ATP-Binding Subunit of Protease Ti (Clp) Can Be Expressed Independently from clpP in Escherichia coli. Biochemical and Biophysical Research Communications, 203(2), pp.798-804. Available at: http://www.sciencedirect.com/science/article/pii/S0006291X84722534.

Yu, X.C. \& Margolin, W., 1999. FtsZ ring clusters in min and partition mutants: role of both the Min system and the nucleoid in regulating FtsZ ring localization. Molecular microbiology, 32(2), pp.315-326.

Zhou, Y. et al., 2001. The RssB response regulator directly targets $\sigma \mathrm{S}$ for degradation by ClpXP. Genes and Development, 15(5), pp.627-637. 
Figure 1: ClpXP structure from E. coli. A ribbon model for hexameric ClpX from E. coli (PDB 3HWS) is depicted in blue above a ribbon model for tetradecameric ClpP protease from E. coli (PDB 1TYF) shown in green (Glynn et al. 2009, Wang et al. 1997). The side view of ClpX and ClpP is shown left, and the top view of ClpX docking ClpP (which opens the ClpP channel) is shown right, and the central channel is clearly visible. The ClpX N-domain is depicted to the right with blue cartoon ovals, demonstrating how the $\mathrm{N}$-domain dimerizes independently and is situated above the AAA+ ClpX domains. 


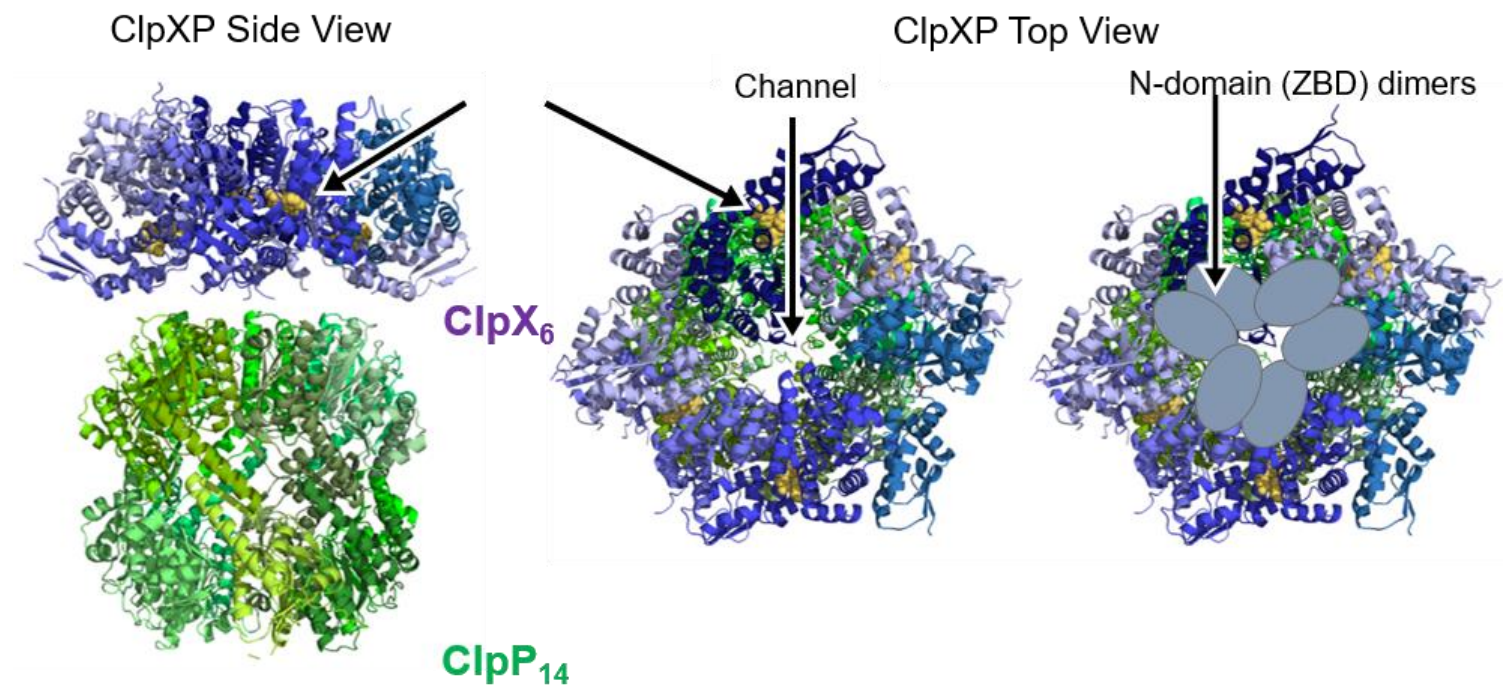

PDB 3HWS, Glynn et al., Cell 2009;

PDB 1TYF, Wang et al., Cell 1997 
Figure 2: Z-ring assembly and FtsZ structure.

(A) In a dividing E. coli cell, a loose network of FtsZ polymers are tethered to the inner face of the cytoplasmic membrane as determined from high-resolution microscopy of Zrings in several bacterial species. (B) FtsZ contains a globular, N-terminal polymerization domain (residues 1-316, shown as a dimer in the purple ribbon model, PDB 1W5A) that binds GTP depicted in yellow, a flexible/unstructured linker region (317-369, dotted line), and a structured C-terminus (370-383, grey cartoon) (Oliva et al. 2004). 
A

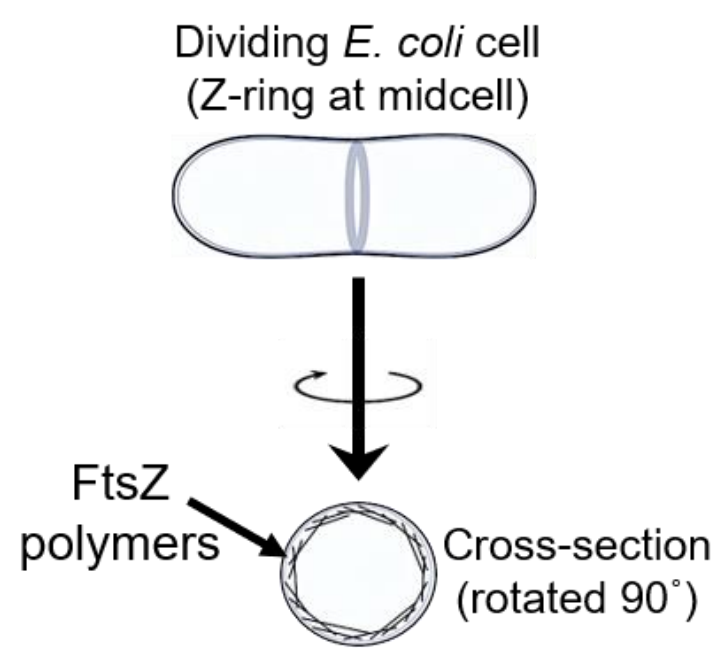

Z-ring Architecture Model

(from high resolution microscopy)

B
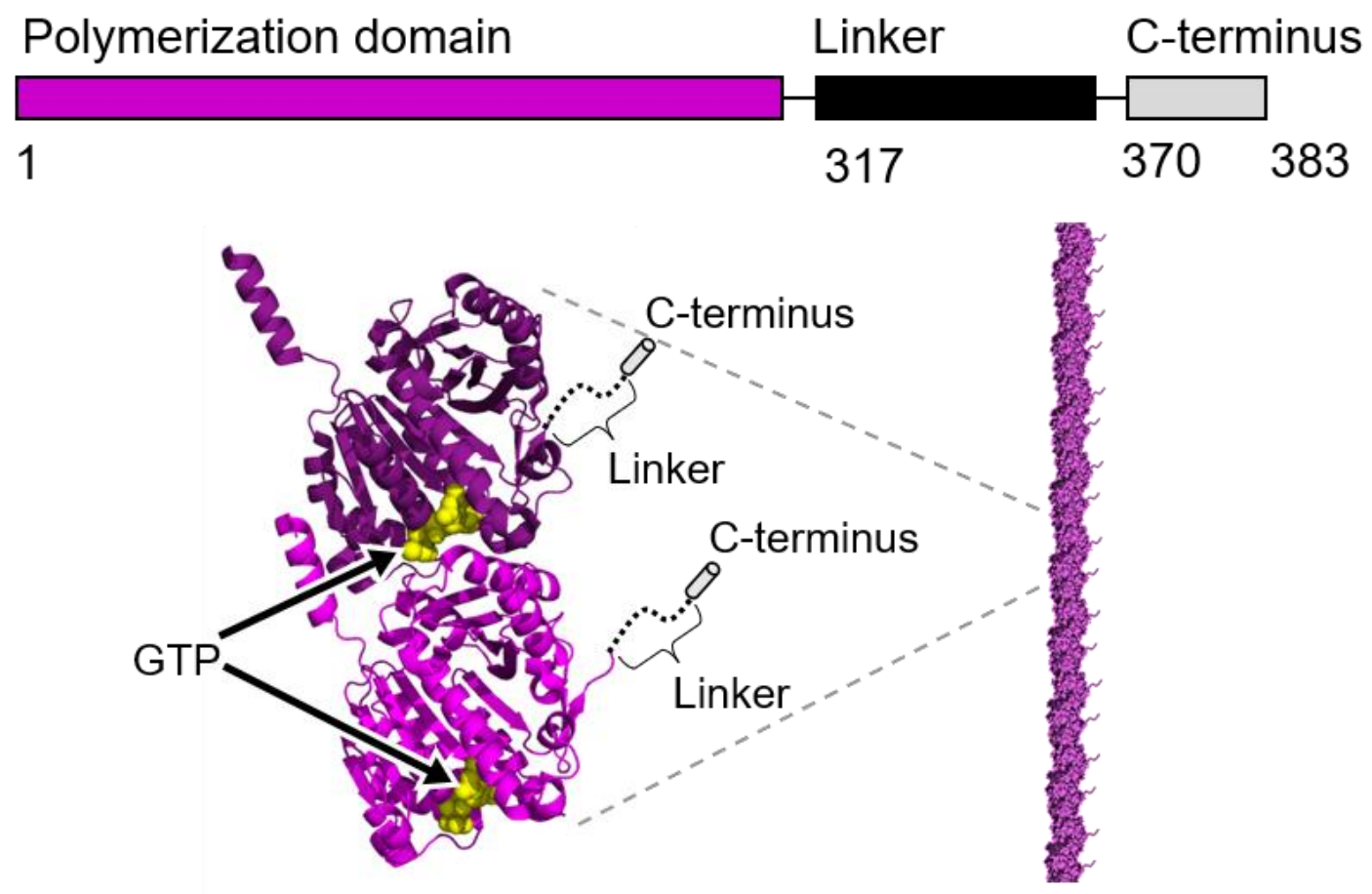

FtsZ Dimer Model

Based on PDB 1W5A, Oliva et al., NSMB 2004 
Figure 3: Recognition of the FtsZ C-terminus by cell division regulators.

Polymer-stabilizing proteins are depicted with light blue ovals, and polymer-disassembly proteins are depicted with red rectangles. ZipA is a polymer-stabilizing protein, ClpXP and MinC disassemble FtsZ polymers or prevent FtsZ polymer assembly, while FtsA has been reported to do both under different conditions (Ma \& Margolin 1999, Pichoff \& Lutkenhaus 2002, Mosyak et al. 2000, Haney et al. 2001, Shen \& Lutkenhaus 2010, Camberg et al. 2014, Viola et al. 2017, Du \& Lutkenhaus 2014, Szwedziak et al. 2012). The conserved region of the FtsZ-Cterminus is underlined, and regulatory proteins are aligned with the C-terminal residues they have been reported to interact with. 


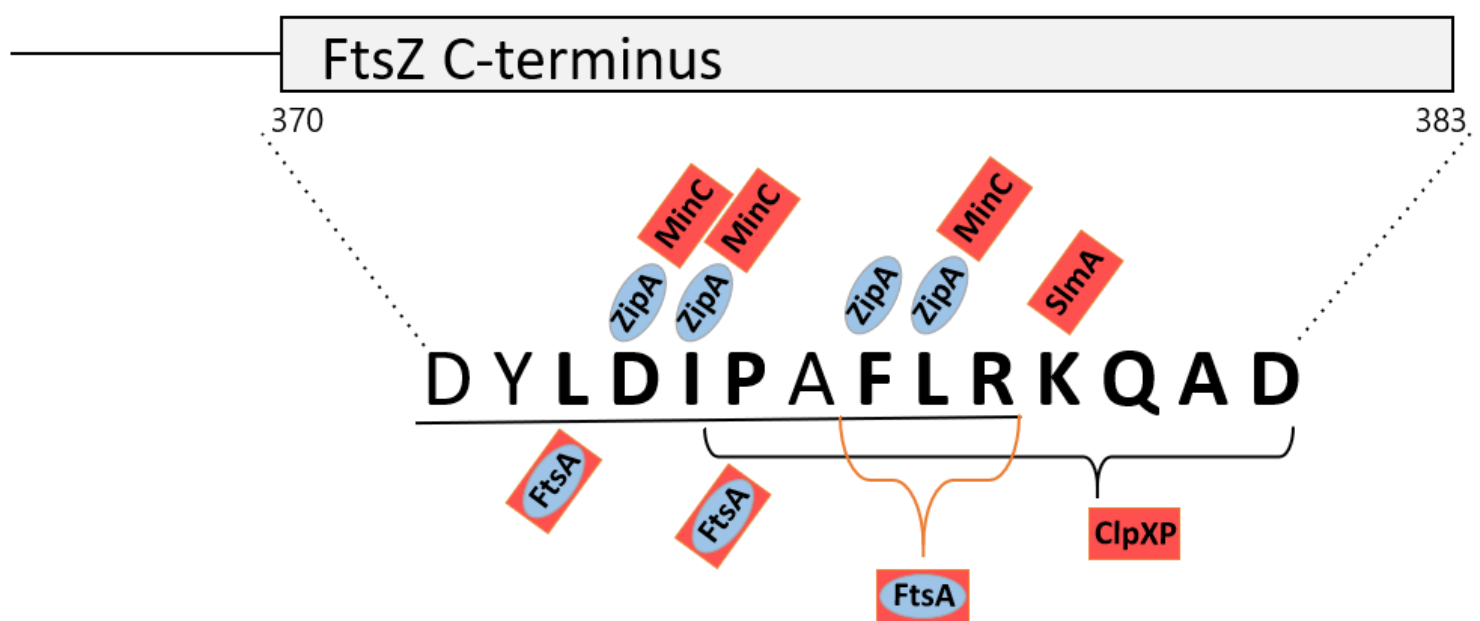


Figure 4: Recognition of FtsZ by ClpXP.

(A) The schematic for the structure of FtsZ shows the globular, N-terminal polymerization domain (residues 1-316, shown in purple), the flexible/unstructured linker region (317-369), and the structured C-terminus (370-383) (Oliva et al. 2004). The regions where $\mathrm{ClpX}$ recognizes FtsZ are shown in teal in the linker region (degron 2, residues 352-358) and yellow at the C-terminal region (degron 1, residues 374-383) (Camberg et al. 2014). A model for degradation of monomers/dimers by ClpXP (B) and for degradation of polymers by $\mathrm{ClpXP}(\mathrm{C})$, where a cross-section of ClpXP is depicted throughout (ClpX in blue, ClpP in green, and FtsZ subunits in purple). ClpX binds and stabilizes an interaction with an FtsZ subunit, then binds and hydrolyzes ATP to power substrate unfolding, which destabilizes the protein substrate $(B, C)$. Unfolding substrates are translocated to the ClpP peptidase, resulting in degradation into small peptides $(\mathrm{B}, \mathrm{C})$. Degradation of monomer/dimer subunits decreases the availability of subunits in the cytosol (B), and degradation within a polymer leads to polymer breakage (C). 
A FtsZ:

Polymerization domain Linker C-terminus

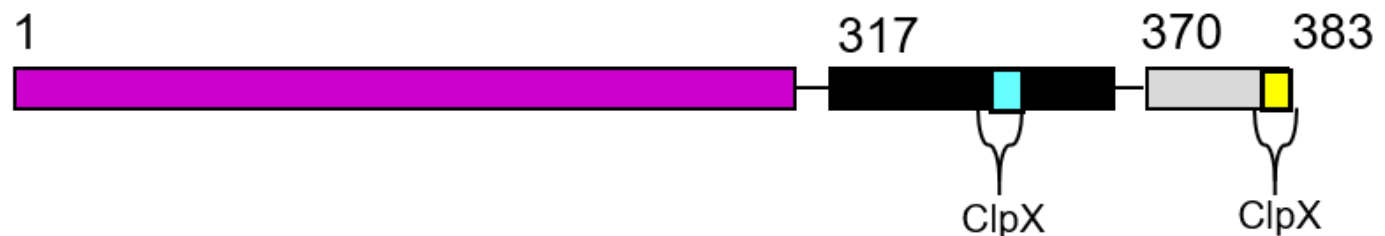

Degron 2 Degron 1

B

Monomer/Dimer:

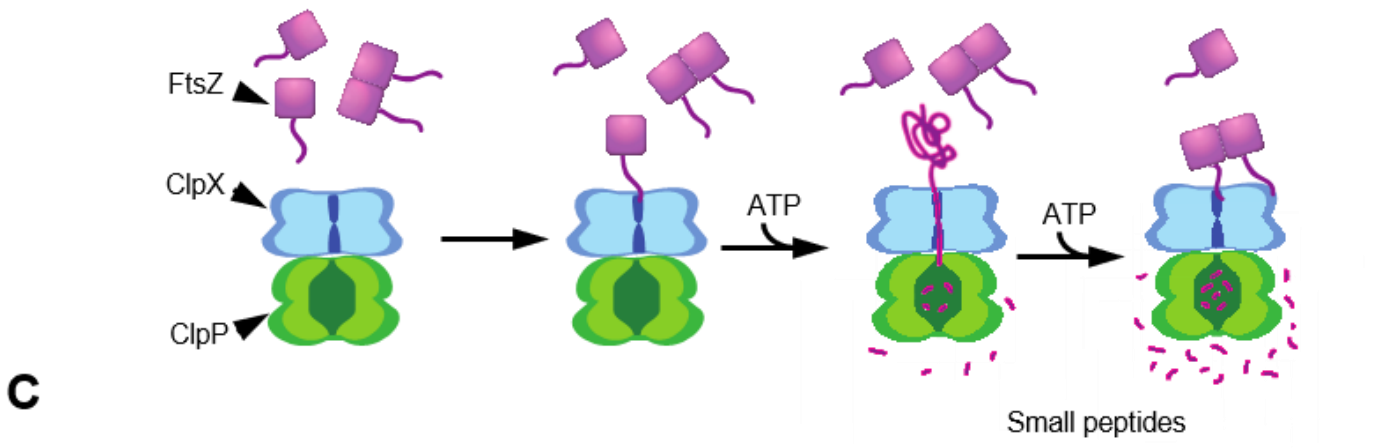

Polymer:
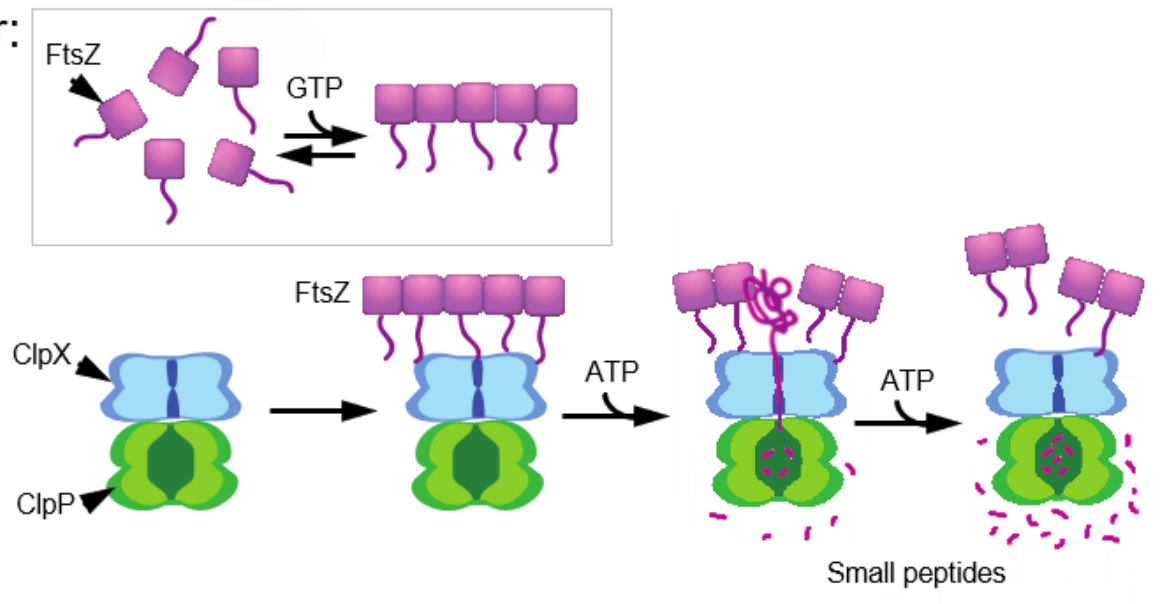
Figure 5: Impact of ClpXP degradation on FtsZ dynamics during cell division.

(A) ClpXP degradation shifts the population of FtsZ towards disassembly (1) or polymer breakage may promote faster polymer remodeling (2), which is consistent with reports for the ClpXP substrate MuA (Abdelhakim et al. 2008; 2010). (B) ClpXP degradation of a small population of FtsZ subunits would support the loose, Z-ring structure which has been reported for various bacterial species based on high-resolution microscopy of Zrings. 
A
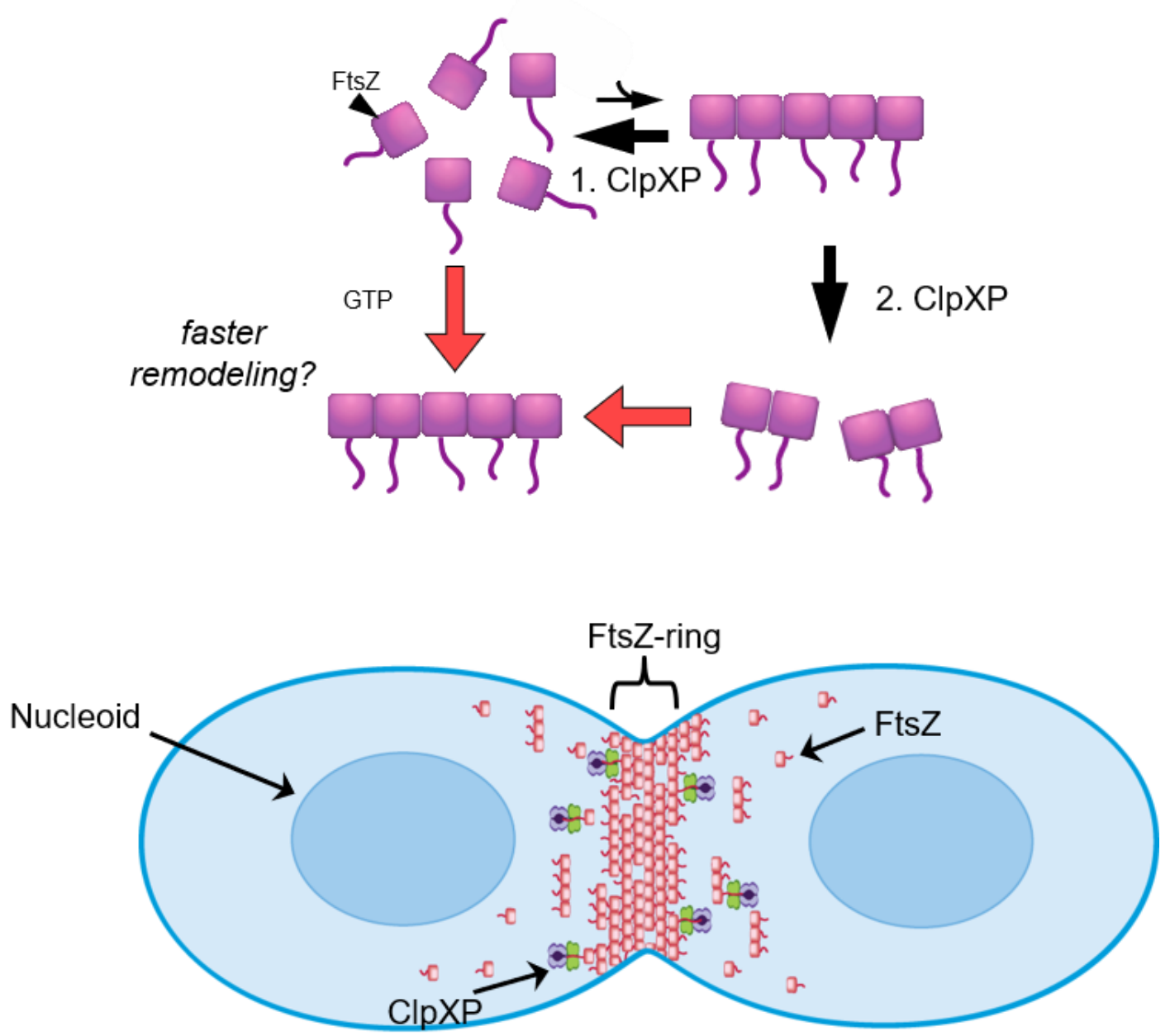


\section{Manuscript II}

Publication status: Published in PLoS One, 2014

Title: Location of dual sites in E. coli FtsZ important for degradation by ClpXP; one at the C-terminus and one in the disordered linker

Authors: *Jodi L. Camberg ${ }^{\mathrm{a}, \mathrm{b}}$, Marissa G. Viola ${ }^{\mathrm{b}}$, Leslie Rea ${ }^{\mathrm{a}}$, Joel R. Hoskins ${ }^{\mathrm{a}}$ and Sue Wickner $^{\mathrm{a}}$

\section{Author Affiliations:}

${ }^{a}$ Laboratory of Molecular Biology, National Cancer Institute, National Institutes of Health, Bethesda, MD, USA

${ }^{b}$ Department of Cell and Molecular Biology, College of Environment and Life Sciences, University of Rhode Island, Kingston, RI, USA

*Corresponding author: Jodi L. Camberg, 120 Flagg Road, Kingston, RI, 02881; Tel: (401)874-4961; Email: cambergj@mail.uri.edu

or

Sue Wickner, 37 Convent Drive, Bethesda, MD, 20892; Tel: (301)496-2629; Email: wickners@mail.nih.gov 


\section{ABSTRACT}

ClpXP is a two-component ATP-dependent protease that unfolds and degrades proteins bearing specific recognition signals. One substrate degraded by Escherichia coli ClpXP is FtsZ, an essential cell division protein. FtsZ forms polymers that assemble into a large ring-like structure, termed the Z-ring, during cell division at the site of constriction. The FtsZ monomer is composed of an N-terminal polymerization domain, an unstructured linker region and a $\mathrm{C}$-terminal conserved region. To better understand substrate selection by ClpXP, we engineered FtsZ mutant proteins containing amino acid substitutions or deletions near the FtsZ C-terminus. We identified two discrete regions of FtsZ important for degradation of both FtsZ monomers and polymers by ClpXP in vitro. One region is located 30 residues away from the C-terminus in the unstructured linker region that connects the polymerization domain to the $\mathrm{C}$-terminal region. The other region is near the FtsZ C-terminus and partially overlaps the recognition sites for several other FtsZinteracting proteins, including MinC, ZipA and FtsA. Mutation of either region caused the protein to be more stable and mutation of both caused an additive effect, suggesting that both regions are important. We also observed that in vitro MinC inhibits degradation of FtsZ by ClpXP, suggesting that some of the same residues in the C-terminal site that are important for degradation by ClpXP are important for binding MinC. 


\section{INTRODUCTION}

AAA + ATPases (ATPases associated with various cellular activities) represent a superfamily of ATPases that are present across kingdoms and encompass a variety of cellular functions, including intracellular trafficking, DNA replication, cytokinesis, protein folding and degradation. In Escherichia coli, the AAA+ ATPase ClpX partners with ClpP, a serine protease, to form a two-component ATP-dependent proteolytic machine. The substrate recognition component of the ClpXP protease is ClpX, which is present as a hexameric ring. Hexameric $\mathrm{ClpX}$ associates with $\mathrm{ClpP}$, a barrel-shaped structure composed of two seven-membered rings with an internal proteolytic chamber. Using the energy from ATP hydrolysis, ClpX unfolds polypeptides and threads the polypeptide chain through the central channel of $\mathrm{ClpX}$ and into the central proteolytic chamber of ClpP where degradation occurs [1]. The N-domain of ClpX interacts with several substrates directly, including E. coli UmuD and bacteriophage proteins MuA and lambda O protein [2-4]. Some degradation substrates require an adaptor protein for efficient recognition. Adaptor proteins bind specifically to a substrate and to $\mathrm{ClpX}$ to promote substrate engagement and initiation of unfolding by ClpX [5]. For example, the SspB protein enhances degradation of ssrA-tagged substrates by promoting an interaction between ClpX and the ssrA-tag [6].

We previously demonstrated that ClpXP degrades polymerized and nonpolymerized FtsZ in vitro, and the rate of degradation for polymerized FtsZ is faster than non-polymerized FtsZ [7]. FtsZ is recognized by ClpX directly and does not require an 
adaptor protein, however the $\mathrm{N}$-domain of $\mathrm{ClpX}$ is important for degradation of FtsZ [7]. FtsZ, a homolog of the eukaryotic protein tubulin, is essential in E. coli and forms a large structure with ring-like architecture at the nascent division site; this structure is referred to as the Z-ring [8]. Formation of the Z-ring precedes constriction at the septum and cell separation. Evidence suggests that the Z-ring may be comprised of dynamic FtsZ protein filaments that are bundled and tethered to the inner face of the cytoplasmic membrane through direct interactions with membrane-associated proteins FtsA and ZipA [8].

FtsZ is a GTPase and assembles into dynamic polymers in the presence of GTP in vitro [9]. Several proteins in E. coli bind to FtsZ and influence the dynamic assembly of FtsZ fibers [8]. These include ZipA, ZapA and ZapC, which stabilize FtsZ polymers from disassembly and promote lateral bundling $[8,10,11]$. Conversely, E. coli proteins MinC, SlmA and ClpXP destabilize FtsZ fibers and promote disassembly [7,12-14]. Of these modulators of FtsZ assembly, several, including FtsA, ZipA, ClpXP and MinC, have been demonstrated to interact with a region of FtsZ near the C-terminus that contains a highly conserved sequence, referred to as the conserved core $[7,8,15,16]$. MinC has been suggested to have a second interaction with FtsZ near the GTP-binding site at the interface between adjacent protomers [17]. MinC functions to prevent lateral bundling of FtsZ fibers and longitudinal polymer assembly [18].

Although it degrades FtsZ in vivo and in vitro, ClpXP is not an essential protein in E. coli for cell division or other cellular functions [19]. However, it modulates cell division by lowering the concentration of FtsZ, thereby shifting the dynamic equilibrium 
away from the polymerized form of FtsZ [7]. Several other modulators of FtsZ assembly are also not essential, including the FtsZ-associated proteins ZapA, ZapB, ZapC and ZapD [20]. Unlike the other modulators of FtsZ assembly, ClpXP is not a dedicated cell division protein and degrades many diverse protein substrates [21].

In the present study we report the identification of two regions near the FtsZ Cterminus that are important for degradation of FtsZ by ClpXP. One region is located 30 amino acids away from the $\mathrm{C}$-terminus in an unstructured linker and the other region includes residues near the $\mathrm{C}$-terminus. Our results suggest that $\mathrm{ClpX}$ recognizes FtsZ through dual contacts and residues in both regions are important for degradation by ClpXP. Although FtsZ degradation does not require an adaptor, degradation is inhibited when the SspB adaptor binding site on the ClpX N-domain is occupied by a peptide containing $10 \mathrm{C}$-terminal amino acid residues of SspB. We also demonstrate in vitro that FtsZ degradation is reduced in the presence of excess MinC, which is consistent with both MinC and ClpXP interacting with an overlapping region of FtsZ near the Cterminus.

\section{EXPERIMENTAL PROCEDURES}

\section{Bacterial strains and plasmids}

E. coli strains and plasmids used in this study are listed in Supporting Information (Table

S1). Bacteria were grown in Lennox (LB) liquid broth at $30^{\circ} \mathrm{C}$ in the presence of 
ampicillin and arabinose, where indicated. The chromosomal araE promoter was replaced in MG1655 by $\mathrm{P} 1$ transduction with the constitutive promoter $\mathrm{P}_{\mathrm{CP} 18}$ to regulate expression of the high-capacity transporter ( $\mathrm{araE}$ ) [22,23]. Plasmids encoding FtsZ mutant proteins were constructed by site-directed mutagenesis of pBAD-FtsZ, pGfp-FtsZ and pEt-FtsZ using the Quik-Site II Mutagenesis kit (Agilent) [7,24,25].

\section{Proteins and Peptides}

E. coli FtsZ [7], ClpX [26], ClpP [27] and GFP-ssrA [28] proteins were expressed and purified as described previously. $\mathrm{ClpX}(\mathrm{E} 185 \mathrm{Q})$ was purified like wild type $\mathrm{ClpX}$ [26]. The C-terminal SspB peptide, XB, with the sequence NH2-RGGRPALRVVK$\mathrm{COOH}$ was purchased from Life Technologies. MinC was cloned into vector pET-24b (EMD-Millipore). Expression was induced in BL21( $\lambda \mathrm{DE} 3)$ cells (EMD-Millipore USA) (Table S1) at $30^{\circ} \mathrm{C}$ by adding $0.5 \mathrm{mM} \beta$-D-isopropyl-thiogalactoside after cells reached an O.D. 600 of 1.2. After $3 \mathrm{~h}$ of induction, cells were harvested by centrifugation at $6,000 \mathrm{x}$ $g$ for $20 \mathrm{~min}$, resuspended in $25 \mathrm{mM}$ Tris- $\mathrm{HCl}, \mathrm{pH} 8.0,50 \mathrm{mM} \mathrm{KCl}, 10 \%$ glycerol, $1 \mathrm{mM}$ EDTA and $1 \mathrm{mM}$ TCEP [tris(2-carboxyethyl)phosphine], and then lysed by French press. The cell lysate was centrifuged at $35,000 \mathrm{x} g$ for $30 \mathrm{~min}$ at $4^{\circ} \mathrm{C}$. MinC was purified from the soluble cell extract by chromatography on a Q sepharose column. Bound proteins were eluted with a $\mathrm{KCl}$ gradient $(50-600 \mathrm{mM})$. Fractions containing $\mathrm{MinC}$ were fractionated on a sephacryl S-100 column equilibrated with $25 \mathrm{mM}$ Tris-HCl, $\mathrm{pH}$ 7.5, 100 $\mathrm{mM} \mathrm{KCl}, 10 \%$ glycerol and $1 \mathrm{mM}$ TCEP. Protein concentrations are reported for ClpX hexamers, $\mathrm{ClpP}$ tetradecamers, MinC dimers and FtsZ monomers. 
FtsZ mutant proteins were expressed in E. coli BL21 ( $\lambda$ DE3) and purified like wild type FtsZ as described [7]. To incorporate fluorescent labels into active FtsZ wild type or mutant proteins, stable polymers were formed by adding GTP in the presence of $\mathrm{CaCl}_{2}$, then labeled with Alexa fluor 350, 488 or 670 succinimidyl ester (Life Technologies) to a degree of labeling ranging from $0.5-5.0 \mathrm{~mol} / \mathrm{mol}$ as described [29]. Fluorescent FtsZ wild type and mutant proteins were depolymerized as described to obtain active labeled protein [30].

\section{Degradation Reactions}

Fluorescent wild type or mutant FtsZ $(10 \mu \mathrm{M})$ was incubated in assembly buffer [50 mM MES (morpholino-ethane-sulfonic acid), $\mathrm{pH} 6.5,50 \mathrm{mM} \mathrm{KCl}$ and $10 \mathrm{mM}$ $\left.\mathrm{MgCl}_{2}\right]$ with $25 \mu \mathrm{g} / \mathrm{ml}$, acetate kinase and $15 \mathrm{mM}$ acetyl phosphate. Where indicated, 2 $\mathrm{mM}$ GTP was added and reactions were incubated for $3 \mathrm{~min}$ at room temperature to promote FtsZ polymer formation. After polymerization, ClpX, ClpP and $4 \mathrm{mM}$ ATP were added, where indicated, at the start of the degradation reaction. After incubation for 30 min, degradation reactions were stopped by the addition of $25 \mathrm{mM}$ EDTA. Reactions were filtered on Nanosep ultrafiltration membranes (Pall Life Sciences) (MWCO 10 $\mathrm{kDa}$ ), pre-washed with $100 \mathrm{mM} \mathrm{NaCl}$ containing $0.01 \%$ Triton $\mathrm{X}-100$, by centrifugation at $16,000 \times \mathrm{g}$ for $20 \mathrm{~min}$. Total fluorescence of peptides in the eluent was measured using

a Cary fluorometer. Background correction was made by subtracting the fluorescence of the eluting volume from reactions containing fluorescent FtsZ wild type or mutant 
protein, but without ClpXP.

Degradation reactions containing FtsZ in the presence of MinC were performed as above, except the final concentration of FtsZ present in the reaction was $5 \mu \mathrm{M}$. MinC was included in the FtsZ degradation reaction at final concentrations of 2, 5, 10 and $20 \mu \mathrm{M}$.

GFP-ssrA degradation was monitored by measuring the loss of fluorescence with time as described in reactions containing ClpX, ClpP, ATP and MinC, where indicated [28].

\section{Functional assays of FtsZ mutant proteins in vivo}

E. coli strain MCZ84, containing the chromosomal ftsZ84 gene, was transformed with arabinose inducible pBAD expression plasmids listed in Table S1 encoding FtsZ wild type and mutant proteins. Strains were grown overnight at $30^{\circ} \mathrm{C}$ in Lennox Broth containing ampicillin $\left(100 \mu \mathrm{g} \mathrm{ml}^{-1}\right)$. Stationary phase cultures were diluted 1:100 in LB broth containing $0.05 \% \mathrm{NaCl}, 0.02 \%$ arabinose and ampicillin, and grown at $42{ }^{\circ} \mathrm{C}$ for 4 h. Colony forming units (CFU) were determined by dilution plating onto LB agar containing ampicillin, then growing the colonies overnight at the permissive temperature.

\section{Assembly Characterization of FtsZ mutant proteins}

FtsZ wild type and mutant proteins were assayed for GTP hydrolysis using the 
phosphate detection reagent Biomol Green (Enzo Life Sciences). Reactions containing 5 $\mu \mathrm{M}$ FtsZ and $1 \mathrm{mM}$ GTP in assembly buffer were incubated at $30^{\circ} \mathrm{C}$ for $15 \mathrm{~min}$. The amount of free phosphate was measured in reactions at 0 and 15 min by comparison to a phosphate standard curve. Rates were calculated by measuring the amount of free phosphate released during the incubation period.

To measure polymerization by light scattering, wild type and mutant FtsZ polymers were assembled in reactions ( $80 \mu \mathrm{l})$ containing assembly buffer and $8 \mu \mathrm{M}$ FtsZ. Polymerization was monitored with time after the addition of $1 \mathrm{mM}$ GTP by light scattering using a Cary Eclipse fluorescence spectrophotometer with excitation and emission wavelengths set to $450 \mathrm{~nm}$ and $5 \mathrm{~nm}$ slit widths. Baseline readings were collected for two min, GTP was added and light scattering was measured for 30 min. FtsZ wild type and mutant polymers were visualized by negative staining with uranyl acetate and electron microscopy as described [48].

\section{Polymerization Assays with MinC}

Fluorescent FtsZ polymers were assembled by mixing $5 \mu \mathrm{M}$ fluorescence-labeled FtsZ in assembly buffer in the presence of $25 \mu \mathrm{g} / \mathrm{ml}$ acetate kinase and $15 \mathrm{mM}$ acetyl phosphate. GTP $(2 \mathrm{mM})$ was added and reactions were incubated for $3 \mathrm{~min}$. Where indicated MinC, ClpX, ClpP and $4 \mathrm{mM}$ ATP were added, and reactions were incubated for $10 \mathrm{~min}$. FtsZ polymers were collected by centrifugation for $30 \mathrm{~min}$ at $129,000 \mathrm{x} g$ at 
$23{ }^{\circ} \mathrm{C}$. Supernatants and pellets were resuspended in assembly buffer containing $0.1 \mathrm{M}$ $\mathrm{NaCl}$ and $0.005 \%$ Triton $\mathrm{X}-100$. Total fluorescence was measured in supernatants and pellets.

\section{Co-sedimentation Assays}

FtsZ polymers were assembled by adding $2 \mathrm{mM}$ GTP to reactions containing assembly buffer, $25 \mu \mathrm{g} / \mathrm{ml}$ acetate kinase and $15 \mathrm{mM}$ acetyl phosphate with $250 \mathrm{pmol}$ FtsZ wild type or mutant protein. After incubating the reaction for 3 min to allow for GTP-dependent polymerization, $1.5,5.0$ or $12.5 \mathrm{pmol} \mathrm{ClpX}(\mathrm{E} 185 \mathrm{Q})$ and $4 \mathrm{mM}$ ATP were added to the reaction. The final reaction volume was $25 \mu 1$. After incubating for $10 \mathrm{~min}$, reactions were centrifuged at $129,000 \times \mathrm{g}$ for $30 \mathrm{~min}$. Supernatants and pellets, resuspended in an equivalent volume of assembly buffer, were analyzed by SDS-PAGE and Coomassie staining. The relative amounts of $\mathrm{ClpX}(\mathrm{E} 185 \mathrm{Q})$ in supernatant and pellet fractions were quantified by densitometry, and pmol of $\mathrm{ClpX}(\mathrm{E} 185 \mathrm{Q})$ in the pellet fraction was calculated. Values were background-corrected by subtracting the total pmol of $\mathrm{ClpX}(\mathrm{E} 185 \mathrm{Q})$ present in the pellet under identical conditions but omitting GTP and normalized to the amount of polymerized wild type or mutant FtsZ detected, which ranged from 99 to 174 pmol.

\section{RESULTS}




\section{The C-terminal region of $\mathrm{FtsZ}$ is important for recognition and degradation by ClpXP}

The region of FtsZ referred to as the "conserved C-terminal core", which includes residues 370 through 379 [16], is located near the C-terminus (Fig. 1A). Residues 380 through 383 of FtsZ are less conserved and referred to as the C-terminal variable region [31]. The conserved core region contains residues that interact with several cell division proteins, including FtsA, ZipA and MinC [7,8,15,16]. In addition residues within 18 amino acids of the C-terminus, 366 to 383, are important for degradation by ClpXP [7]. To elucidate the residues within this region of FtsZ that are important for ClpX recognition we constructed FtsZ deletion and substitution mutants (Fig. 1A). FtsZ( $\Delta_{380-}$ 383) is a deletion mutant that lacks the C-terminal four amino acid variable region and $\operatorname{FtsZ}\left(\Delta_{375-383}\right)$ is a deletion mutant lacking the C-terminal nine amino acids. We also engineered substitution mutations at two positively charged residues near the $\mathrm{C}$-terminus, R379E and K380A. We determined the rates of degradation of fluorescent wild type and mutant FtsZ by ClpXP by monitoring the appearance of degradation products using an ultrafiltration assay. As we previously observed, FtsZ was degraded at an approximately 2-fold faster rate in the presence of GTP, the condition that promotes FtsZ polymerization, than in the absence of GTP $\left(0.20 \mathrm{~min}^{-1}\right.$ and $0.11 \mathrm{~min}^{-1}$ in the presence and absence of GTP, respectively) (Fig. 1B). However, FtsZ mutant proteins lacking either four or nine residues from the FtsZ C-terminus were degraded at $\sim 75 \%$ reduced rates compared to wild type FtsZ in the presence of GTP and $\sim 65 \%$ reduced rates compared to wild type FtsZ in the absence of GTP. The reduction in the degradation rates 
of $\operatorname{FtsZ}\left(\Delta_{380-383}\right)$ and $\operatorname{FtsZ}\left(\Delta_{375-383}\right)$ were similar to the reduction in the degradation rate previously observed for $\operatorname{FtsZ}\left(\Delta_{366-383}\right)$ (Fig. 1B) [7]. We also observed that FtsZ substitution mutants FtsZ(R379E) and FtsZ(K380A) were degraded by ClpXP at $\sim 75 \%$ and $\sim 55 \%$ reduced rates, respectively, compared to wild type FtsZ in both the presence and absence of GTP (Fig. 1B). These results indicate that the positively charged residues R379 and K380 of FtsZ are important for degradation of FtsZ by ClpXP. Taken together, our results suggest that $\mathrm{ClpX}$ recognizes FtsZ through a C-terminal recognition motif and further implicates amino acid residues R379 and K380 as important for degradation by ClpXP.

Structural models of an E. coli FtsZ C-terminal peptide containing residues 367 through 383, which cocrystallized with the FtsZ-binding domain of ZipA, show a nine amino acid linear alpha helix at the FtsZ C-terminus (Fig. 1C) [32]. Amino acid residues R379 and K380 are located within the C-terminal helix, and the side chains extend outward. Cocrystallization of this region with FtsA from Thermatoga maritima showed an alternative configuration, with the helix containing a 90 degree bend, which could suggest that the FtsZ tail may be capable of adopting different conformations [33]. In the absence of structural information concerning the configuration of the E. coli FtsZ Cterminal tail with ClpX, this region has been illustrated based on the E. coli model with ZipA (Fig. 1C) [32].

Our results indicate that the FtsZ C-terminal region, containing positively charged amino acid residues R379 and K380, is important for degradation of FtsZ by ClpXP. 
ClpX is known to recognize the C-termini of substrates that contain one of two distinct recognition motifs; one motif, referred to as $\mathrm{C}$ motif-1, resembles the C-terminal LAA residues of the ssrA tag sequence, and a second motif, referred to as $\mathrm{C}$ motif-2, resembles the C-terminal sequence of the degradation substrate from bacteriophage $\mathrm{Mu}, \mathrm{MuA}$, a DNA transposase [21]. An alignment of the FtsZ C-terminus with the C-terminal 10 amino acids from substrates that contain recognition tags bearing similarity to the $\mathrm{C}$ motif-2 consensus motif (R/H-x-K/R-K- $\Phi$ with $\mathrm{x}$ representing any amino acid and $\Phi$ representing a hydrophobic amino acid residue) is shown in Fig. 1D [21]. The alignment shows similarities between the C-terminus of FtsZ and other ClpXP degradation substrates, including K380 and the nearby hydrophobic amino acid A382. The reduced rate of degradation observed for the mutant protein $\operatorname{FtsZ}\left(\Delta_{380-383}\right)$, which is missing both $\mathrm{K} 380$ and $\mathrm{A} 382$, compared to wild type FtsZ is consistent with the suggestion that these residues are important for degradation by ClpXP.

Additional substitution mutations in the conserved alpha-helical region near the FtsZ C-terminus were constructed and tested for degradation by ClpXP (Fig. S1). We observed $\sim 50 \%$ reduced rates of degradation of two mutant proteins FtsZ(F377A) and FtsZ(P375G) in the presence of GTP, compared to wild type FtsZ; however, we did not observe similar reductions when GTP was omitted (Fig. S1). One mutant protein, FtsZ(L378A), was degraded at a 1.9-fold faster rate than wild type FtsZ in the absence of GTP, but not in the presence of GTP. Together, these results suggest that amino acid residues in the vicinity of the recognition motif may modulate the rate of degradation in the presence or absence of GTP. 


\section{The linker region of FtsZ also contains residues important for degradation by ClpXP}

Our observation that deletion of 4, 9, or 18 residues from the FtsZ C-terminus reduced degradation by ClpXP but did not abolish it (Fig. 1B), suggests that additional residues in FtsZ may also participate in a ClpX interaction. Therefore we examined the linker region of FtsZ, residues 317 through 369, between the polymerization domain and the conserved C-terminal domain for an additional site of interaction for ClpX (Fig. 2A). The linker region overall is poorly conserved in bacteria, and structure prediction algorithms suggest that the linker has little secondary structure. Erickson and colleagues recently showed that the linker region is an intrinsically disordered peptide and because it could be replaced with almost any sequence of similar length, it likely functions as a bridge, linking the FtsZ polymerization domain to the $\mathrm{C}$-terminal protein interaction sites via a flexible tether [34]. By sequence examination, we identified a 10-residue motif in the linker region (349 QEQKPVAKVV 358) that contains 60\% homology to the ClpXP recognition signal in $\mathrm{Mu}$ repressor, however the region did not strictly adhere to the $\mathrm{C}$ motif-2 consensus motif (R/H-x-K/R-K- $\Phi)$ (Fig. 2A). To probe this region further and test if residues in this potential site are also important for the interaction with ClpX, we constructed several triple alanine substitutions in the region, shown in Fig. 2B. We compared rates of degradation of the FtsZ linker mutant proteins to wild type FtsZ in the presence and absence of GTP (Fig. 2C). We observed that two of the mutant proteins we constructed, FtsZ(352AAA) and FtsZ(356AAA), were degraded at $60-75 \%$ slower rates 
than wild type FtsZ in both the presence and absence of GTP. However, a nearby triple alanine substitution mutant, 349AAA, was degraded at a rate similar as wild type FtsZ (Fig. 2C).

To test if the two regions we identified are both important for degradation, we

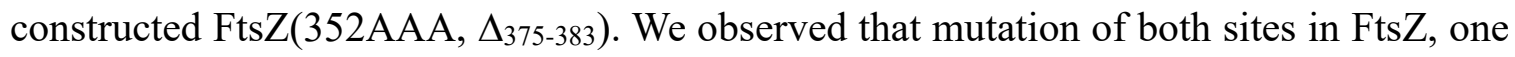
in the linker and the other near the C-terminus, abolished degradation by ClpXP in the presence or absence of GTP (Fig. 2C). Taken together, our data demonstrate that two regions of FtsZ separated by approximately 20 residues promote the recognition and degradation of FtsZ. Disruption of both regions in FtsZ prevents degradation by ClpXP.

\section{FtsZ mutant proteins hydrolyze GTP and exhibit GTP-dependent polymerization}

To test if FtsZ mutant proteins are functional for GTP-dependent assembly and GTP hydrolysis, we performed a characterization of FtsZ mutant proteins in vitro. As expected, all of the mutant proteins hydrolyze GTP. Many of the FtsZ mutant proteins exhibit rates of GTP hydrolysis similar to wild type FtsZ $\left(4.9 \pm 0.6 \mathrm{~min}^{-1}\right)$, however we observed a $\sim 40 \%$ slower rate of GTP hydrolysis by FtsZ(349AAA) and FtsZ(352AAA, $\Delta_{375-383)}$ compared to wild type FtsZ (Fig. 3A). In addition all of the mutant proteins showed an increase in light scatter when GTP was added indicating that they polymerize in a GTP-dependent manner (Fig. 3B). However, the increase in light scatter upon addition of GTP was $\sim 30-70 \%$ less with FtsZ(R379E), FtsZ(K380A), FtsZ $\left(\Delta_{380-383}\right)$,

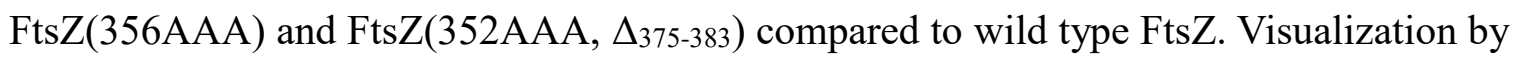


electron microscopy and negative staining showed that all of the FtsZ mutant proteins tested formed single-stranded filaments in the presence of GTP (Fig. S2), including FtsZ(352AAA, $\Delta_{375-383)}$ which showed reduced GTPase activity and GTP-stimulated light scatter. We also observed fiber bundles in addition to single stranded filaments by electron microscopy for mutant proteins FtsZ(R379E), FtsZ(352AAA) and FtsZ(352AAA, $\Delta_{375-383)}$.

\section{A region in the ClpX N-domain overlapping the SspB binding site is important for FtsZ recognition and degradation by ClpXP}

We next wanted to investigate the region of $\mathrm{ClpX} \mathrm{N-domain} \mathrm{that} \mathrm{interacts} \mathrm{with}$ FtsZ. The N-domain of ClpX, comprised of residues 1 through 61, is important for recognition and degradation of FtsZ [7]. The ClpX N-domain is also essential for recognition and degradation of lambda $\mathrm{O}$ protein and adaptor-mediated substrate recognition and degradation, including ssrA-tagged proteins in the presence of adaptor

protein $\mathrm{SspB}[3,6,35]$. A peptide corresponding to the $10 \mathrm{C}$-terminal amino acids of $\mathrm{SspB}$, referred to as the $\mathrm{XB}$ peptide, binds directly to the $\mathrm{ClpX} \mathrm{N}$-domain and inhibits SspB-stimulated degradation of ssrA tagged substrates [36,37]. The XB peptide also inhibits degradation of lambda $\mathrm{O}$ protein, and structural studies suggest that the lambda $\mathrm{O}$ binding site on ClpX overlaps with the SspB binding site [3,35]. Since the ClpX Ndomain is essential for recognition and degradation of FtsZ by ClpXP [7], we tested if degradation is susceptible to inhibition by the $\mathrm{XB}$ peptide. We monitored degradation of 
FtsZ in the presence of increasing amounts of XB peptide with and without GTP. We observed that addition of XB peptide inhibited degradation of FtsZ in the presence and absence of GTP in a concentration-dependent manner (Fig. 4A). The XB peptide is approximately two-fold more inhibitory against FtsZ degradation in the presence of GTP than in the absence of GTP. These results show that the region of SspB that interacts with the N-domain of ClpX is an inhibitor of FtsZ degradation. They suggest that the FtsZ binding site on $\mathrm{ClpX}$ overlaps with the $\mathrm{SspB}$ adaptor-binding site. These results also demonstrate that FtsZ polymers are more susceptible to inhibition by the peptide than FtsZ monomers.

Next we tested if both regions of FtsZ that promote ClpXP degradation, the linker and the C-terminal region, are susceptible to peptide inhibition by monitoring degradation of FtsZ(352AAA) and FtsZ( $\left.\Delta_{375-383}\right)$ in the presence and absence of the XB peptide. Our results show that degradation of FtsZ(352AAA) and FtsZ( $\left.\Delta_{375-383}\right)$ is inhibited by the XB peptide to a similar extent as wild type FtsZ (Fig. 4B), suggesting that the SspB binding region of the ClpX N-domain is important for interaction with both of the FtsZ degradation signals, the one in the linker and the one near the C-terminus.

\section{Association of CIpX with wild type and mutant FtsZ polymers}

Having shown that FtsZ degradation by ClpXP involves two regions of FtsZ (Fig. $1 \mathrm{~B}$ and $2 \mathrm{C}$ ), we wanted to determine if the FtsZ mutant proteins that are poorly degraded by ClpXP are defective in an interaction with ClpX. To test this, we performed a co- 
pelleting assay to monitor the association of polymerized FtsZ wild type or mutant protein with an ATP hydrolysis defective mutant of ClpX, ClpX(E185Q), which can form stable interactions with substrates in the presence of ATP [38]. We observed that ClpX(E185Q) co-pelleted with wild type FtsZ polymers in a concentration-dependent manner (Fig. 5). ClpX(E185Q) also associated with FtsZ(356AAA) polymers. However,

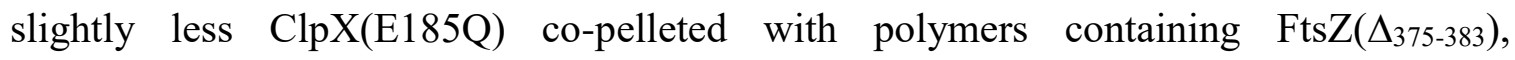
FtsZ(352AAA) or FtsZ(352AAA, $\Delta_{375-383)}$ than wild type FtsZ, suggesting that these mutants are slightly defective for the association with ClpX(E185Q).

\section{ClpXP and MinC compete for FtsZ in vitro}

MinC interacts with FtsZ in the C-terminal domain and isoleucine 374 has been shown to be important for this interaction [15]. Therefore if ClpX associates with FtsZ near the $\mathrm{C}$-terminus, an interaction with MinC could potentially mask residues important for recognition by $\mathrm{ClpX}$ and prevent degradation in vitro. To test if MinC competes with ClpXP for FtsZ in vitro, we monitored degradation of fluorescent FtsZ by ClpXP in the presence of increasing amounts of MinC. We observed that MinC inhibited FtsZ degradation with $80 \%$ inhibition resulting from a four-fold excess of MinC dimer over FtsZ monomer (Fig. 6A). In a control experiment, we observed that degradation of GFPssrA by ClpXP was not inhibited by MinC (Fig. S3). One interpretation of our results is that there is competition between $\mathrm{ClpXP}$ and MinC in vitro for binding the C-terminal domain of FtsZ. 
Both MinC and ClpXP have independently been shown to destabilize FtsZ polymers in vitro leading to polymer disassembly $[7,18]$. In our in vitro competition experiment, we observed inhibition of FtsZ degradation when MinC was in excess over FtsZ. However, in vivo FtsZ is in large excess over MinC, based on estimates of 10,000 and 400 molecules of FtsZ and MinC, respectively, per cell $[39,40]$. Consequently, multiple modulators of FtsZ assembly are likely acting independently and at the same time on the large amount of FtsZ inside the cell. Therefore, to test if MinC and ClpXP act concurrently to disrupt FtsZ polymers in vitro when MinC and ClpXP are under limiting conditions, we monitored FtsZ polymer abundance after incubation with ClpXP and MinC. MinC was added first to preassembled FtsZ polymers, then ClpXP and ATP were added to the reaction. After a short incubation, the remaining FtsZ polymers were collected by centrifugation and quantified. The supernatant fractions, which were not quantified, contained mixtures of FtsZ monomers and dimers, as well as products of the degradation reaction when ClpXP and ATP were included. In control experiments, the addition of either ClpXP or MinC to FtsZ polymers caused a reduction in FtsZ polymer abundance by $33 \%$ for ClpXP and 25\% for MinC (Fig. 6B). When ClpXP and MinC were both included in the reaction, there was a $65 \%$ reduction of FtsZ polymers. The reduction was similar to the additive value of the individual contributions of ClpXP and MinC, or approximately $60 \%$, and dependent on the presence of ATP. These results suggest that under our conditions, MinC and ClpXP perform independent and concurrent disruption activities on FtsZ polymers in vitro. 


\section{FtsZ C-terminal mutants are defective for function in vivo}

In E. coli, $\operatorname{clp} X$ and $\operatorname{clpP}$ are dispensable for growth and genetic deletion of $\operatorname{clp} X$ or $\operatorname{clpP}$ is not associated with division defects [7,19]. In addition ClpXP-dependent phenotypes have not been described. Since the C-terminal region of FtsZ is essential for interactions with known cell division proteins as well as ClpXP, it is likely that any growth defects exhibited by our FtsZ mutant proteins are caused by failure of FtsZ to interact with essential cell division proteins including MinC, FtsA or ZipA. However, we tested FtsZ mutant proteins in vivo to determine if mutations in regions of FtsZ important for ClpXP degradation are also associated with defects in FtsZ function. We expressed each mutant FtsZ protein from a plasmid in an ftsZ84 temperature sensitive strain [41] and measured the number of CFUs after incubation at $42^{\circ} \mathrm{C}$. In control experiments, expression of wild type FtsZ from a plasmid supported growth of $f$ ts $Z 84$ cells at $42^{\circ} \mathrm{C}$, while cultures of cells carrying the control vector were unable to grow (Fig. 7). Expression of FtsZ( $\Delta_{375-383)}$ and FtsZ(R379E) did not support growth and cells expressing $\operatorname{FtsZ}\left(\Delta_{380-383}\right)$ were partially functional, as expected since residues in this region are important for interactions with both ZipA and FtsA. Cells expressing FtsZ(K380A) grew slightly better than those expressing wild type FtsZ, while substitution mutations in residues 375 through 378 impaired FtsZ function in vivo (Fig. S4A), as previously reported [16]. Expression of the FtsZ linker mutant proteins FtsZ(349AAA), FtsZ(352AAA) and FtsZ(356AAA) support growth of ftsZ84 cells at $42^{\circ} \mathrm{C}$, similar to cells expressing wild type FtsZ (Fig. 7). However, cells expressing the double mutant FtsZ(352AAA, $\Delta_{375-383)}$ were unable to grow. The cell growth defects 
detected in this assay likely reflect changes in interactions with essential cell division proteins such as MinC, FtsA or ZipA. In addition, no defects were detected in our assay in cells expressing FtsZ(352AAA) or FtsZ(356AAA), which are defective for degradation by ClpXP in vitro.

Next, we tested whether the FtsZ mutant proteins localize to Z-rings in wild type cells by expressing each FtsZ mutant protein as a green fluorescent protein (GFP) fusion from a plasmid. All GFP-FtsZ mutant proteins tested assembled into ring-like structures in dividing cells (Fig. S5). Expression of GFP-FtsZ proteins containing triple alanine substitutions in the linker region showed normal Z-rings and no obvious cell division defects. In contrast, many of the C-terminal mutations, including R379E and $\Delta_{375-383 \text {, }}$ were associated with mild cell filamentation and multiple Z-rings in some cells (Fig. S5 and Fig. S4B). Filamentation and Z-ring localization was observed in cells that expressed GFP-FtsZ proteins containing mutations at residues 375, 376, 377 and 378 (Fig. S4B) [16]. Cells expressing GFP-FtsZ( $\left.\Delta_{380-383}\right)$ or GFP-FtsZ(K380A) exhibited normal Z-ring formation and were not filamentous.

\section{DISCUSSION}

In this study, we have identified two regions near the FtsZ C-terminus that are important for proteolysis by ClpXP. The C-terminal end of FtsZ harbors a motif that resembles the C motif-2 family of ClpX recognition motifs proposed in Flynn, et al [21]. Like the ClpXP substrate MuA, the C-terminus of FtsZ contains positively charged 
residues, R379 and K380, which are important for degradation by ClpXP [4]. Our results further show that there is a second site important for degradation by ClpXP that is located 30 residues from the C-terminus of FtsZ in a highly flexible and unstructured linker region [34]. Another ClpXP substrate, MuA, also utilizes additional contacts to stabilize the ClpX-interaction, and the presence of multiple ClpX recognition sites in FtsZ may serve a similar function [4]. In FtsZ, both sites can function independently since both FtsZ(352AAA) and FtsZ( $\left.\Delta_{375-383}\right)$ are partially defective and inhibited by the SspB Cterminal peptide (Fig. 1B, 2C and 4B). A change in the rate of degradation could result from impaired recognition, unfolding, translocation into $\mathrm{ClpP}$, or proteolytic cleavage. Co-pelleting assays between FtsZ mutants and an ATP hydrolysis-defective mutant of ClpX (Fig. 5) are consistent with the suggestion that the reduced rates of degradation may be due to defective recognition. However in our assay FtsZ(352AAA, $\Delta_{375-383)}$ was only partially defective for an interaction with $\mathrm{ClpX}(\mathrm{E} 185 \mathrm{Q})$, which could suggest that substrate engagement is impaired.

GTP-dependent polymerization of FtsZ shown here, and previously with the GTP-analog GMPCPP [7], enhances the rate of degradation of FtsZ (Fig. 1B), suggesting that the GTP-bound or polymer form of FtsZ may be recognized more efficiently. This could be due to exposure of additional contacts upon GTP-binding or polymerization, or due to enhanced association supported by multivalent interactions with polymerized FtsZ. Interestingly, C-terminal substitution mutants of FtsZ at residues P375, A376, or F377 are not stimulated for degradation in the presence of GTP compared to the absence of GTP (Fig. S1), suggesting that these residues could be important for stabilizing interactions 
with ClpX; however, GTP enhances the rate of degradation of FtsZ $\left(\Delta_{375-383}\right)$, which does not contain residues 375 through 377 , by $\sim 40 \%$ (Fig. 1). Multimerization may be important for enhancing degradation of low affinity substrates by ClpXP by increasing the local concentration of recognition sites and therefore promoting an interaction with ClpX. In our studies with FtsZ, we observed that the rate of degradation is maximal when both the $\mathrm{C}$-terminal and linker regions are present and FtsZ is incubated under conditions that promote polymerization (Figs. $1 \mathrm{~B}$ and $2 \mathrm{C}$ ). Moreover, additional interactions between FtsZ and ClpX, which contains six N-domains per hexamer, may stabilize the interaction or function as a tether to promote additional recognition events.

We observed that FtsZ(R379E), which is poorly degraded by ClpXP in vitro, causes a severe growth defect (Fig. 7 and S5). Since there are no cell growth phenotypes associated with deletion of $c l p X$ or $c l p P$ in wild type $E$. coli, this result suggests that this residue is important for interactions with cell division proteins, such as MinC or FtsA, in addition to ClpX. Other substitution and deletion mutations introduced in the C-terminal conserved region of FtsZ also caused severe functional defects when expressed in ftsZ84 cells and when expressed as GFP fusion proteins (Fig. 7, S4 and S5). In contrast, expression of FtsZ linker mutants FtsZ(352AAA) and FtsZ(356AAA), which are partially defective for degradation by ClpXP in vitro, in ftsZ84 cells grown at the restrictive temperature did not result in obvious functional defects (Fig. 7). A recent study also reported no defects associated with the replacement of residues in the linker region [34].

The C-terminal variable region of FtsZ, which contains the last six residues of 
FtsZ, from B. subtilis has been shown to promote the lateral bundling of FtsZ polymers [31]. In contrast to the results with $B$. subtilis FtsZ, neither the variable region nor the unstructured linker region of E. coli FtsZ has been shown to promote bundling of filaments [31,34]. The FtsZ C-terminal mutant proteins examined in our study form single-stranded polymers, however we observed several pairs or bundles of filaments by electron microscopy (Fig. S2), but they were not the dominant species observed.

Several proteins in the cell, both essential and non-essential, influence the dynamic assembly and disassembly of FtsZ. The major inhibitor of FtsZ polymerization in the bacterial cell is MinC. Our data demonstrate that like MinC, ClpXP is also capable of promoting disruption of FtsZ polymers (Fig. 6B). MinC binds to FtsZ and prevents assembly at the subunit interface and lateral interactions between FtsZ polymers $[17,18,42]$. Although it has been reported that ClpX can inhibit FtsZ polymerization in vitro under certain conditions, our previously published results indicate that FtsZ polymer disassembly activity requires ATP-dependent degradation [7,43]. When MinC and ClpXP are in limiting concentrations compared to FtsZ and not in competition, then they function simultaneously to promote polymer disassembly (Fig. 6B). However, we observed that degradation of FtsZ by ClpXP is reduced by $80 \%$ in the presence of excess MinC, suggesting that $\mathrm{MinC}$ and $\mathrm{ClpX}$ are in competition and recognize overlapping regions of the FtsZ C-terminus. Recently, it was also reported that ZipA can similarly protect FtsZ from degradation by ClpXP, likely through preventing access to the FtsZ Cterminus [44]. Degradation of FtsZ by ClpXP occurs more efficiently under conditions that promote FtsZ polymerization (Fig. 1B) [7]. Therefore disassembly of FtsZ polymers 
by MinC could also contribute to the reduced FtsZ degradation observed in vitro in the presence of MinC.

FtsZ was initially identified as a ClpXP substrate in E. coli in a proteomics study to isolate kinetically-trapped ClpP complexes from cell lysates [21]. Recently, a similar proteomics study in Staphylococcus aureus, also identified FtsZ as a substrate for ClpPmediated degradation [45]. Direct interactions between ClpX and FtsZ have also been reported in Bacillus subtilis and Mycobacterium tuberculosis, although FtsZ does not appear to be degraded by ClpXP in these organisms [46,47]. In E. coli, the physiological effect of inhibiting FtsZ degradation in a cell where division is partially defective, such as the $f$ tsZ84 strain, is beneficial, resulting in partial suppression of the phenotype when $c l p X$ or $c l p P$ is deleted [25]. The role of FtsZ degradation during the division process in a wild type cell is less clear, however modest overexpression of ClpXP enhances FtsZ degradation and causes cellular filamentation associated with defective division [7]. Our results demonstrate that the specific degradation of FtsZ by ClpXP occurs through a complex recognition mechanism that is modulated by FtsZ conformation and may be impacted by the presence of other cell division proteins, including MinC and ZipA, and thus provides a mechanism for the cell to modulate division through proteolysis of FtsZ.

\section{ACKNOWLEDGEMENTS}

We thank Shannon Doyle, Danielle Johnston and Olivier Genest for helpful discussions. We thank the reviewers for their insightful comments and helpful 
suggestions. We also thank Sherri Rennoll and Gabriella Johnson for laboratory assistance and Carol Ayala at Rhode Island Hospital for expert technical assistance with the electron microscope. Fluorescence and DIC microscopy was performed using the Rhode Island Genomics and Sequencing Center, which is supported in part by the National Science Foundation under EPSCoR Grants Nos. 0554548 \& EPS-1004057. 


\section{REFERENCES}

1. Sauer RT, Baker TA (2011) AAA+ proteases: ATP-fueled machines of protein destruction. Annu. Rev. Biochem. 80: 587-612.

2. Neher SB, Sauer RT, Baker TA (2003) Distinct peptide signals in the UmuD and UmuD' subunits of $\mathrm{UmuD} / \mathrm{D}^{\prime}$ mediate tethering and substrate processing by the $\mathrm{ClpXP}$ protease. Proc. Natl. Acad. Sci. U S A 100: 13219-13224.

3. Thibault G, Houry WA (2012) Role of the N-terminal domain of the chaperone ClpX in the recognition and degradation of lambda phage protein O. J. Phys. Chem. B. 116: 67176724.

4. Abdelhakim AH, Oakes EC, Sauer RT, Baker TA (2008) Unique contacts direct highpriority recognition of the tetrameric $\mathrm{Mu}$ transposase-DNA complex by the AAA+ unfoldase ClpX. Mol. Cell 30: 39-50.

5. Kirstein J, Moliere N, Dougan DA, Turgay K (2009) Adapting the machine: adaptor proteins for Hsp100/Clp and AAA+ proteases. Nat. Rev. Microbiol. 7: 589-599.

6. Dougan DA, Weber-Ban E, Bukau B (2003) Targeted delivery of an ssrA-tagged substrate by the adaptor protein $\mathrm{SspB}$ to its cognate $\mathrm{AAA}+$ protein $\mathrm{ClpX}$. Mol. Cell 12: 373-380.

7. Camberg JL, Hoskins JR, Wickner S (2009) ClpXP protease degrades the cytoskeletal protein, FtsZ, and modulates FtsZ polymer dynamics. Proc. Natl. Acad. Sci. U S A 106: 10614-10619.

8. Adams DW, Errington J (2009) Bacterial cell division: assembly, maintenance and disassembly of the Z ring. Nat. Rev. Microbiol. 7: 642-653.

9. Michie KA, Lowe J (2006) Dynamic filaments of the bacterial cytoskeleton. Annu. 
Rev. Biochem. 75: 467-492.

10. Durand-Heredia JM, Yu HH, De Carlo S, Lesser CF, Janakiraman A (2011) Identification and characterization of ZapC, a stabilizer of the FtsZ ring in Escherichia coli. J. Bacteriol. 193: 1405-1413.

11. Hale CA, Shiomi D, Liu B, Bernhardt TG, Margolin W, et al. (2011) Identification of Escherichia coli ZapC (YcbW) as a component of the division apparatus that binds and bundles FtsZ polymers. J. Bacteriol. 193: 1393-1404.

12. Lutkenhaus J (2007) Assembly dynamics of the bacterial MinCDE system and spatial regulation of the $\mathrm{Z}$ ring. Annu. Rev. Biochem. 76: 539-562.

13. Cho H, McManus HR, Dove SL, Bernhardt TG (2011) Nucleoid occlusion factor SlmA is a DNA-activated FtsZ polymerization antagonist. Proc. Natl. Acad. Sci. U S A 108: 3773-3778.

14. Tonthat NK, Arold ST, Pickering BF, Van Dyke MW, Liang S, et al. (2011) Molecular mechanism by which the nucleoid occlusion factor, SlmA, keeps cytokinesis in check. EMBO J. 30: 154-164.

15. Shen B, Lutkenhaus J (2009) The conserved C-terminal tail of FtsZ is required for the septal localization and division inhibitory activity of $\operatorname{MinC}(\mathrm{C}) / \mathrm{MinD}$. Mol. Microbiol. 72: $410-424$.

16. Ma X, Margolin W (1999) Genetic and functional analyses of the conserved Cterminal core domain of Escherichia coli FtsZ. J. Bacteriol. 181: 7531-7544.

17. Shen B, Lutkenhaus J (2010) Examination of the interaction between FtsZ and MinCN in E. coli suggests how MinC disrupts Z rings. Mol. Microbiol. 75: 1285-1298.

18. Dajkovic A, Lan G, Sun SX, Wirtz D, Lutkenhaus J (2008) MinC spatially controls 
bacterial cytokinesis by antagonizing the scaffolding function of FtsZ. Curr. Biol. 18: $235-244$.

19. Gottesman S, Clark WP, de Crecy-Lagard V, Maurizi MR (1993) ClpX, an alternative subunit for the ATP-dependent Clp protease of Escherichia coli. Sequence and in vivo activities. J. Biol. Chem. 268: 22618-22626.

20. Egan AJ, Vollmer W (2013) The physiology of bacterial cell division. Annals N. Y. Acad. Sci. 1277: 8-28.

21. Flynn JM, Neher SB, Kim YI, Sauer RT, Baker TA (2003) Proteomic discovery of cellular substrates of the ClpXP protease reveals five classes of ClpX-recognition signals. Mol. Cell 11: 671-683.

22. Khlebnikov A, Datsenko KA, Skaug T, Wanner BL, Keasling JD (2001) Homogeneous expression of the $\mathrm{P}(\mathrm{BAD})$ promoter in Escherichia coli by constitutive expression of the low-affinity high-capacity AraE transporter. Microbiology 147: 32413247.

23. Blattner FR, Plunkett G, 3rd, Bloch CA, Perna NT, Burland V, et al. (1997) The complete genome sequence of Escherichia coli K-12. Science 277: 1453-1462.

24. Guzman LM, Belin D, Carson MJ, Beckwith J (1995) Tight regulation, modulation, and high-level expression by vectors containing the arabinose PBAD promoter. J. Bacteriol. 177: 4121-4130.

25. Camberg JL, Hoskins JR, Wickner S (2011) The interplay of ClpXP with the cell division machinery in Escherichia coli. J. Bacteriol. 193: 1911-1918.

26. Grimaud R, Kessel M, Beuron F, Steven AC, Maurizi MR (1998) Enzymatic and structural similarities between the Escherichia coli ATP- dependent proteases, ClpXP and 
ClpAP. J. Biol. Chem. 273: 12476-12481.

27. Maurizi MR, Thompson MW, Singh SK, Kim SH (1994) Endopeptidase Clp: ATPdependent Clp protease from Escherichia coli. Methods Enzymol. 244: 314-331.

28. Singh SK, Grimaud R, Hoskins JR, Wickner S, Maurizi MR (2000) Unfolding and internalization of proteins by the ATP-dependent proteases ClpXP and ClpAP. Proc. Natl. Acad. Sci. U S A 97: 8898-8903.

29. Gonzalez JM, Jimenez M, Velez M, Mingorance J, Andreu JM, et al. (2003) Essential cell division protein FtsZ assembles into one monomer-thick ribbons under conditions resembling the crowded intracellular environment. J. Biol. Chem. 278: 37664-37671.

30. Yu XC, Margolin W (1997) Ca2+-mediated GTP-dependent dynamic assembly of bacterial cell division protein FtsZ into asters and polymer networks in vitro. EMBO J. 16: $5455-5463$.

31. Buske PJ, Levin PA (2012) Extreme C terminus of bacterial cytoskeletal protein FtsZ plays fundamental role in assembly independent of modulatory proteins. J. Biol. Chem. 287: 10945-10957.

32. Mosyak L, Zhang Y, Glasfeld E, Haney S, Stahl M, et al. (2000) The bacterial celldivision protein ZipA and its interaction with an FtsZ fragment revealed by X-ray crystallography. EMBO J. 19: 3179-3191.

33. Szwedziak P, Wang Q, Freund SM, Lowe J (2012) FtsA forms actin-like protofilaments. The EMBO J. 31: 2249-2260.

34. Gardner KA, Moore DA, Erickson HP (2013) The C-terminal linker of Escherichia coli FtsZ functions as an intrinsically disordered peptide. Mol. Microbiol. 89: 264-275. 35. Thibault G, Yudin J, Wong P, Tsitrin V, Sprangers R, et al. (2006) Specificity in 
substrate and cofactor recognition by the $\mathrm{N}$-terminal domain of the chaperone $\mathrm{ClpX}$. Proc. Natl. Acad. Sci. U S A 103: 17724-17729.

36. Park EY, Lee BG, Hong SB, Kim HW, Jeon H, et al. (2007) Structural basis of SspBtail recognition by the zinc binding domain of ClpX. J. Mol. Biol. 367: 514-526.

37. Wah DA, Levchenko I, Rieckhof GE, Bolon DN, Baker TA, et al. (2003) Flexible linkers leash the substrate binding domain of $\mathrm{SspB}$ to a peptide module that stabilizes delivery complexes with the AAA+ClpXP protease. Mol. Cell 12: 355-363.

38. Hersch GL, Burton RE, Bolon DN, Baker TA, Sauer RT (2005) Asymmetric interactions of ATP with the AAA + ClpX6 unfoldase: allosteric control of a protein machine. Cell 121: 1017-1027.

39. Szeto TH, Rowland SL, King GF (2001) The dimerization function of MinC resides in a structurally autonomous C-terminal domain. J. Bacteriol. 183: 6684-6687.

40. Erickson HP, Anderson DE, Osawa M (2010) FtsZ in bacterial cytokinesis: cytoskeleton and force generator all in one. Microbiol. Mol. Biol. Rev. 74: 504-528.

41. Dai K, Xu Y, Lutkenhaus J (1993) Cloning and characterization of $f t s N$, an essential cell division gene in Escherichia coli isolated as a multicopy suppressor of ftsA12(Ts). J. Bacteriol. 175: 3790-3797.

42. Hu Z, Mukherjee A, Pichoff S, Lutkenhaus J (1999) The MinC component of the division site selection system in Escherichia coli interacts with FtsZ to prevent polymerization. Proc. Natl. Acad. Sci. U S A 96: 14819-14824.

43. Sugimoto S, Yamanaka K, Nishikori S, Miyagi A, Ando T, et al. (2010) AAA+ chaperone ClpX regulates dynamics of prokaryotic cytoskeletal protein FtsZ. J. Biol. Chem. 285: 6648-6657. 
44. Pazos M, Natale P, Vicente M (2012) A specific role for the ZipA protein in cell division: stabilization of the FtsZ protein. J. Biol. Chem. 288: 3219-3226.

45. Feng J, Michalik S, Varming AN, Andersen JH, Albrecht D, et al. (2013) Trapping and Proteomic Identification of Cellular Substrates of the ClpP Protease in Staphylococcus aureus. J. Proteome. Res. 12: 547-558.

46. Haeusser DP, Lee AH, Weart RB, Levin PA (2009) ClpX inhibits FtsZ assembly in a manner that does not require its ATP hydrolysis-dependent chaperone activity. J. Bacteriol. 191: 1986-1991.

47. Dziedzic R, Kiran M, Plocinski P, Ziolkiewicz M, Brzostek A, et al. (2010) Mycobacterium tuberculosis ClpX Interacts with FtsZ and Interferes with FtsZ Assembly. PloS one 5: e11058.

48. Mukherjee A, Lutkenhaus J (1998) Dynamic assembly of FtsZ regulated by GTP hydrolysis. EMBO J. 17: 462-469. 
FIGURE 1. The FtsZ C-terminus is important for ClpXP degradation. A. Linear schematic diagram of FtsZ protein separated into three regions: the polymerization domain (amino acids 1 through 316), the unstructured linker (amino acids 317 through 369) and the C-terminal domain or conserved core region (amino acids 370 through 383 ). The C-terminal FtsZ deletions and substitution mutations used here are presented. B. Comparison of rates of degradation of FtsZ wild type and mutant proteins in the presence and absence of GTP from in vitro degradation reactions containing $10 \mu \mathrm{M}$ wild type or mutant fluorescent FtsZ and $1 \mu \mathrm{M}$ ClpXP. C. Structural model of the C-terminal alphahelical region of FtsZ, residues 367 through 383, that cocrystallized with ZipA (PDB entry 1F47) [32]. Side chains are shown for R379 (red) and K380 (blue). D. Alignment of the C-terminal amino acid sequences of several proteins recognized by ClpX. C-terminal sequences shown belong to the consensus $\mathrm{C}$ motif-2 family of $\mathrm{ClpX}$ recognition tags (R/H-x-K/R-K- $\Phi$ with $\mathrm{x}$ representing any amino acid and $\Phi$ representing a hydrophobic amino acid residue) [21]. In $\mathrm{B}$, data from 3 replicates are presented as mean $\pm \mathrm{SEM}$. 
A
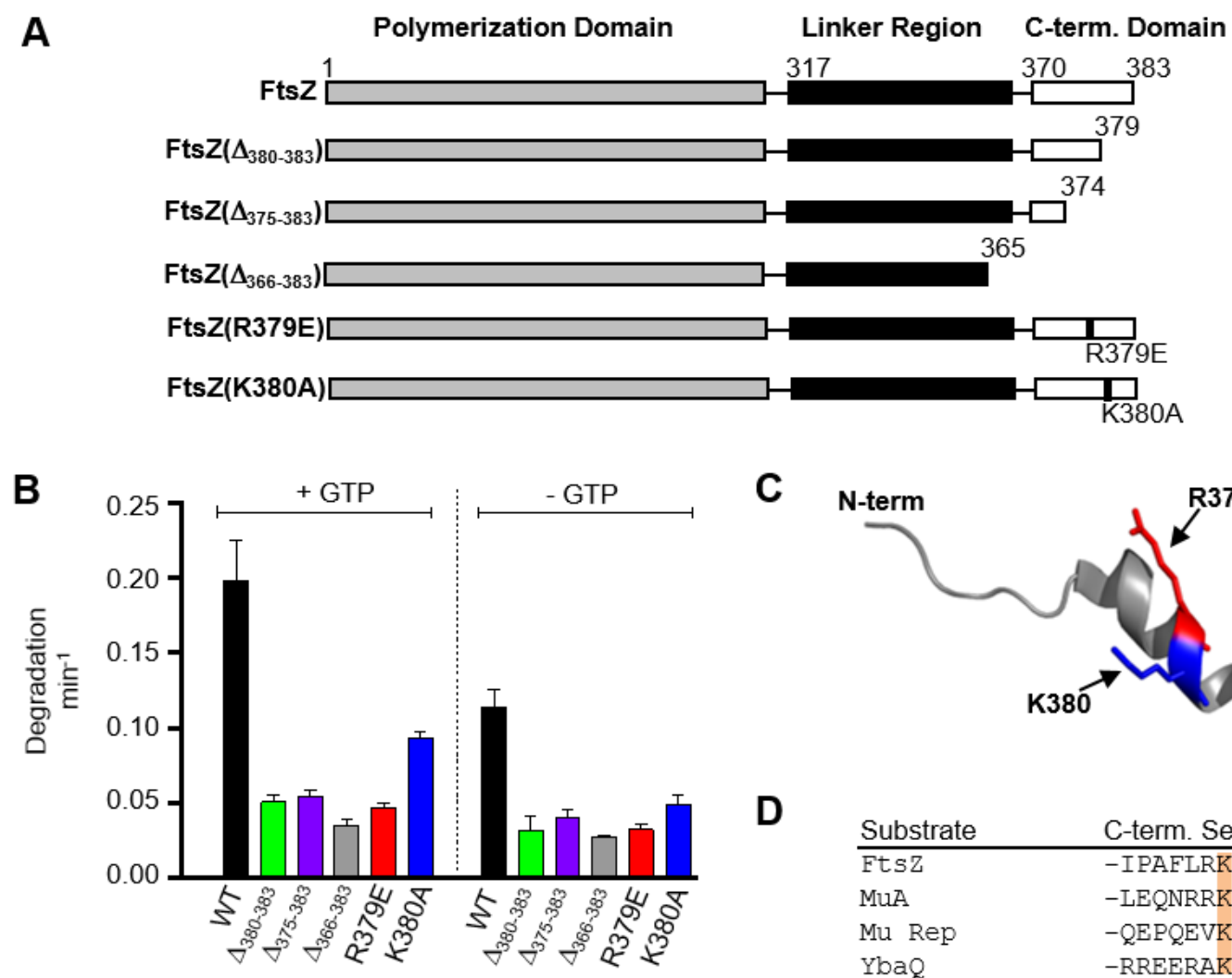

C

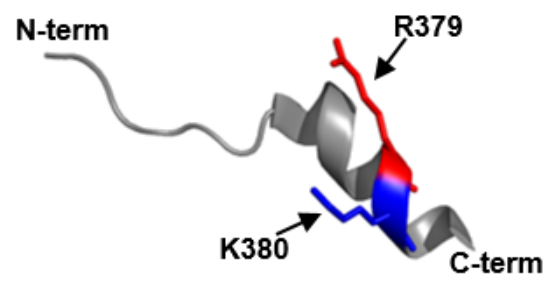

D

\begin{tabular}{ll} 
Substrate & C-term. Sequence \\
\hline FtsZ & -IPAFLRKQAD \\
MuA & -LEQNRRKKAI \\
Mu Rep & -QEPQEVKKAV \\
YbaQ & -RREERAKKVA
\end{tabular}


FIGURE 2. Residues in the linker region of FtsZ are important for degradation by CIpXP. A. Alignment of FtsZ residues 349 through 358 from the linker domain with the C-terminal $\mathrm{ClpX}$ recognition region of $\mathrm{Mu}$ repressor protein from phage $\mathrm{Mu}$. B. Linear schematic diagram of FtsZ showing regions of the linker that were tested by triple alanine scanning mutagenesis of wild type FtsZ and truncated FtsZ( $\left.\Delta_{375-383}\right)$. C. Comparison of rates of degradation of wild type and mutant FtsZ, proteins in the presence and absence of GTP from in vitro degradation reactions containing $10 \mu \mathrm{M}$ wild type or mutant fluorescent FtsZ and $1 \mu \mathrm{M}$ ClpXP. Data from 3 replicates are presented as mean $\pm \mathrm{SEM}$. 


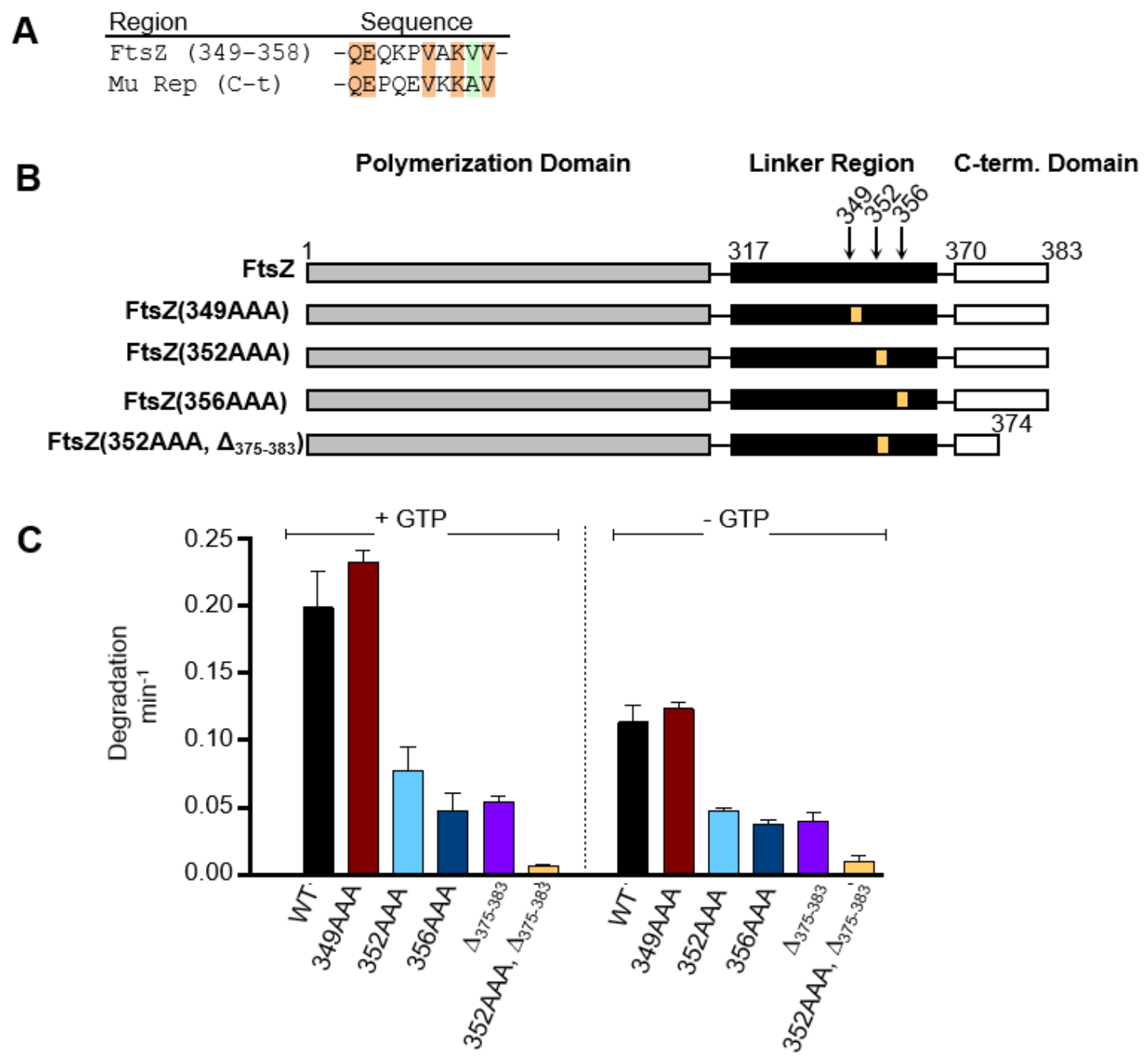


FIGURE 3. FtsZ mutant proteins with C-terminal mutations hydrolyze GTP and assemble into polymers. (A) Rates of GTP hydrolysis were measured in reactions containing FtsZ wild type or mutant $(5 \mu \mathrm{M})$ and GTP $(1 \mathrm{mM})$ as described in Experimental Procedures. Data from 3 replicates are presented as mean \pm SEM. (B) GTP-dependent assembly of FtsZ wild type and mutant proteins $(8 \mu \mathrm{M})$ was monitored by $90^{\circ}$ light scattering as described in Experimental Procedures. A baseline was collected for $2 \mathrm{~min}$, then GTP was added when indicated, to stimulate polymerization. Light scattering was measured for $25 \mathrm{~min}$. Data shown is representative of 3 replicates. 


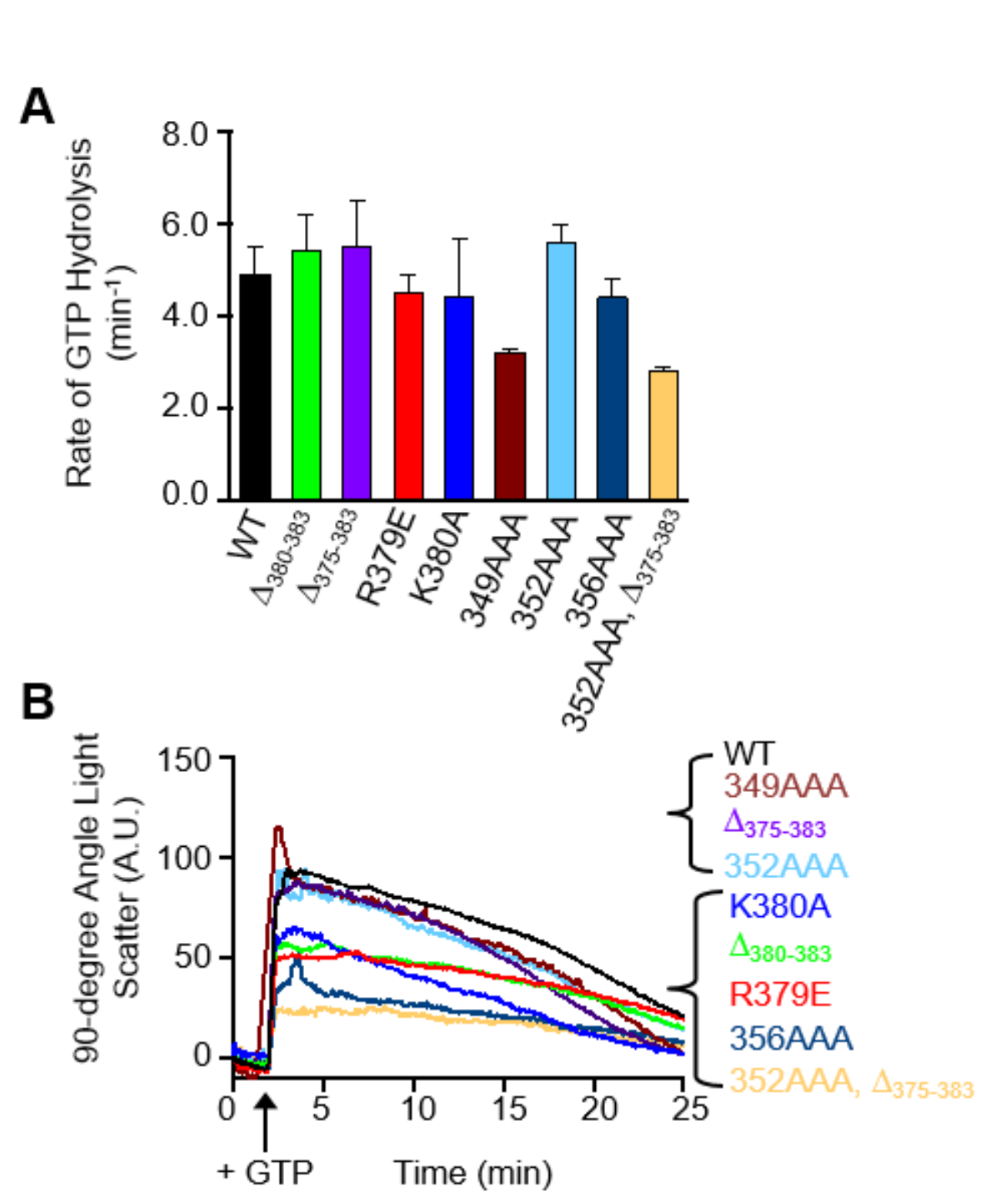


FIGURE 4. A peptide corresponding to the C-terminus of the SspB adaptor inhibits FtsZ degradation. A. Relative rate of FtsZ degradation by ClpXP in the presence and absence of GTP with increasing concentration of SspB peptide $(10,20,40$ or $80 \mu \mathrm{M})$. Relative rate of FtsZ degradation was defined by $\mathrm{V} / \mathrm{V}_{\mathrm{o}}$, where $\mathrm{V}$ is equal to the rate in the presence of $\mathrm{SspB}$ peptide and $\mathrm{V}_{0}$ is equal the rate in the absence of $\mathrm{SspB}$ peptide. Degradation reactions contained $15 \mu \mathrm{M}$ fluorescent FtsZ, $0.75 \mu \mathrm{M}$ ClpXP, ATP and, where indicated, GTP. Data were fit to a nonlinear dose-response inhibitor curve. B. Comparison of relative rates of degradation of wild type FtsZ, FtsZ( $\left.\Delta_{375-383}\right)$ and FtsZ(352AAA) in the presence of 0,20 or $50 \mu \mathrm{M}$ SspB peptide. Reactions contained 10 $\mu \mathrm{M}$ FtsZ wild type or mutant protein, $0.75 \mu \mathrm{M}$ ClpXP with GTP and ATP. In A and B data from 3 replicates are presented as mean \pm SEM. 


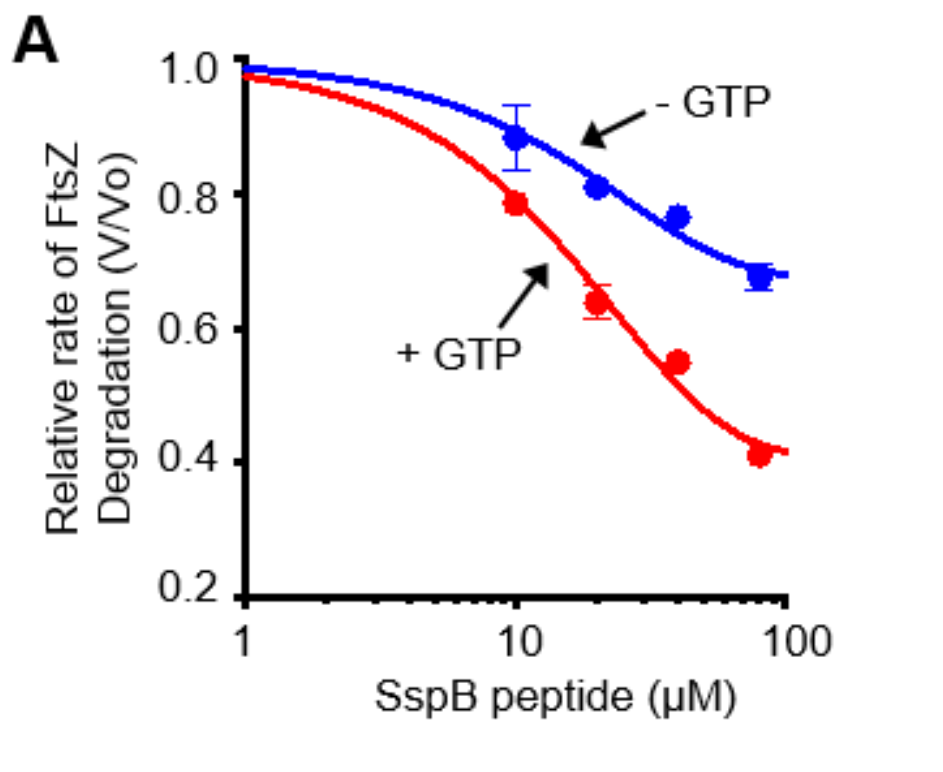

B

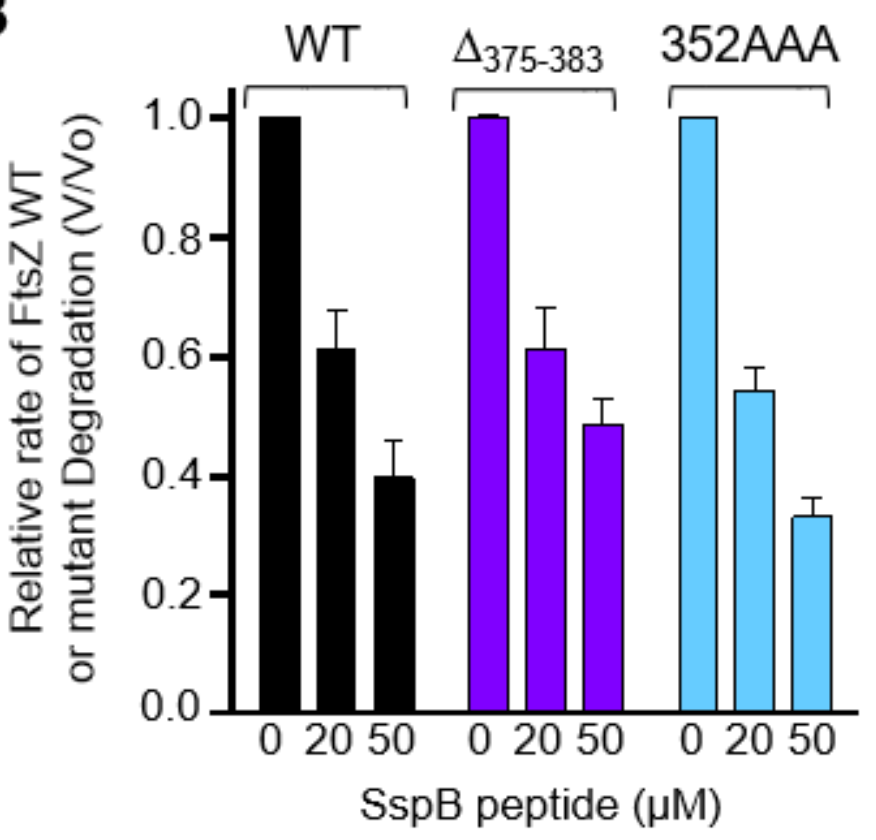


FIGURE 5. Association of FtsZ wild type and mutant polymers with ClpX. The interaction between $\mathrm{ClpX}$ and FtsZ was measured by monitoring the fraction of ClpX

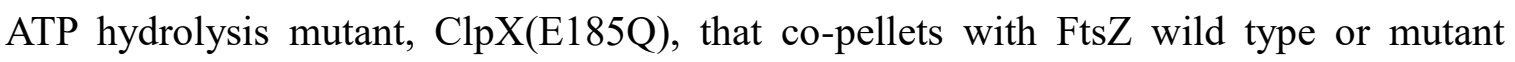
protein in the presence of GTP. Pelleted ClpX(E185Q) and FtsZ was quantified by Coomassie staining of SDS-PAGE gels and densitometry. Data from at least 3 replicates are presented as mean $\pm \mathrm{SEM}$. 


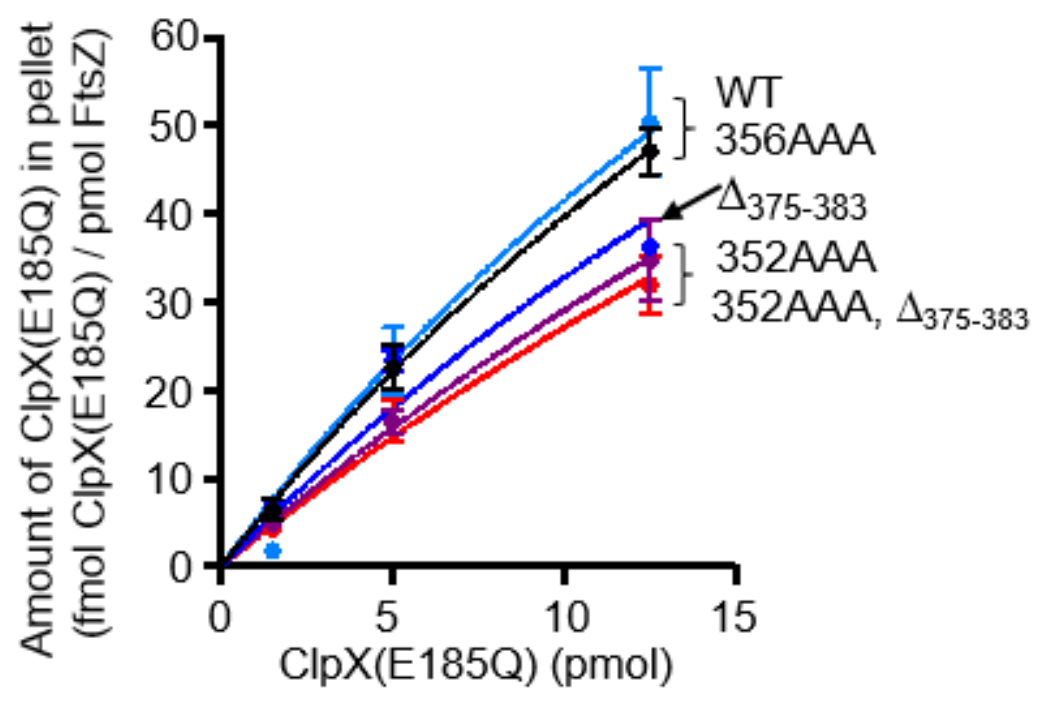


FIGURE 6. MinC competes with CIpXP for FtsZ in vitro. A. MinC was included in degradation reactions containing FtsZ with GTP and ClpXP $(0.5 \mu \mathrm{M})$. B. FtsZ $(5 \mu \mathrm{M}$; $125 \mathrm{pmol} /$ reaction) was first preincubated with GTP $(2 \mathrm{mM})$ and then incubated with MinC $(2 \mu \mathrm{M})$, ClpXP $(0.5 \mu \mathrm{M})$ and ATP, as indicated. FtsZ polymer disruption was monitored by measuring the amount of fluorescent FtsZ present in high-speed centrifugation pellets. In A and B data from 3 replicates are presented as mean $\pm \mathrm{SEM}$. 
A

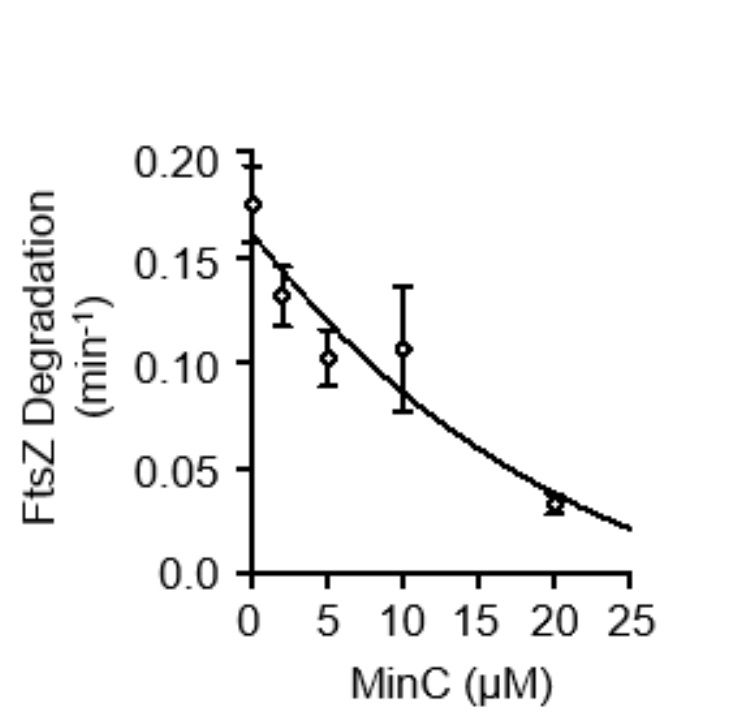

B

$\operatorname{MinC}(\mu \mathrm{M})$

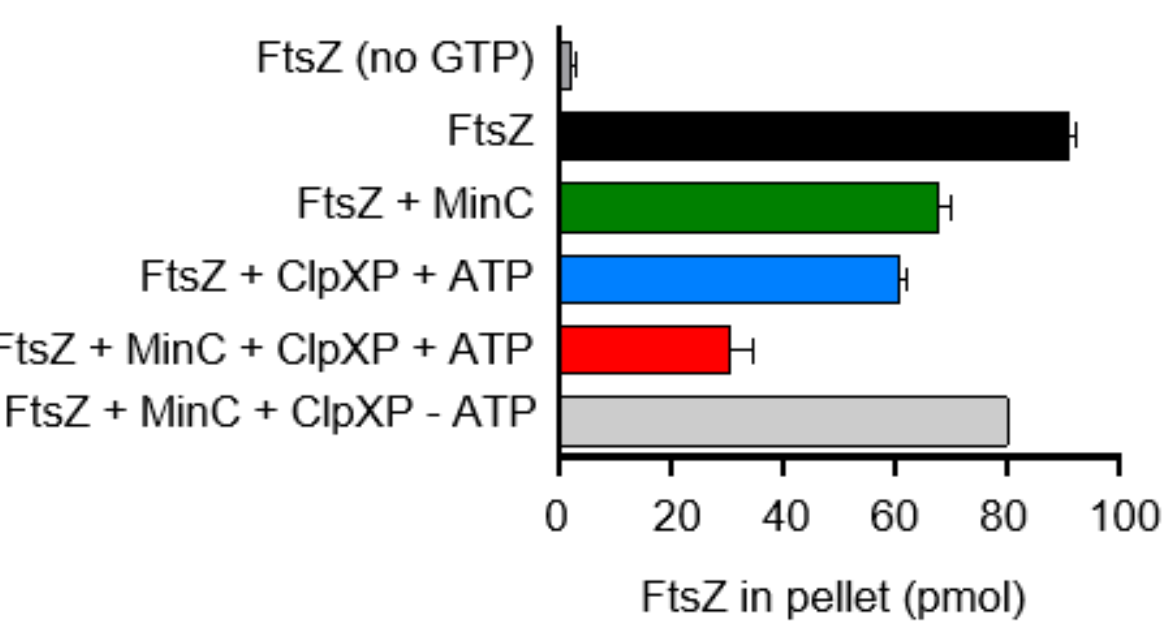


FIGURE 7. Mutations in the FtsZ C-terminal domain impair FtsZ function in vivo.

FtsZ mutant proteins were tested for function in vivo by comparing CFUs of ftsZ84 cells expressing FtsZ mutant proteins after incubation in liquid culture at the restrictive temperature $\left(42^{\circ} \mathrm{C}\right)$ for 4 hours as described in Experimental Procedures. Data from 3 replicates are presented as mean $\pm \mathrm{SEM}$. 


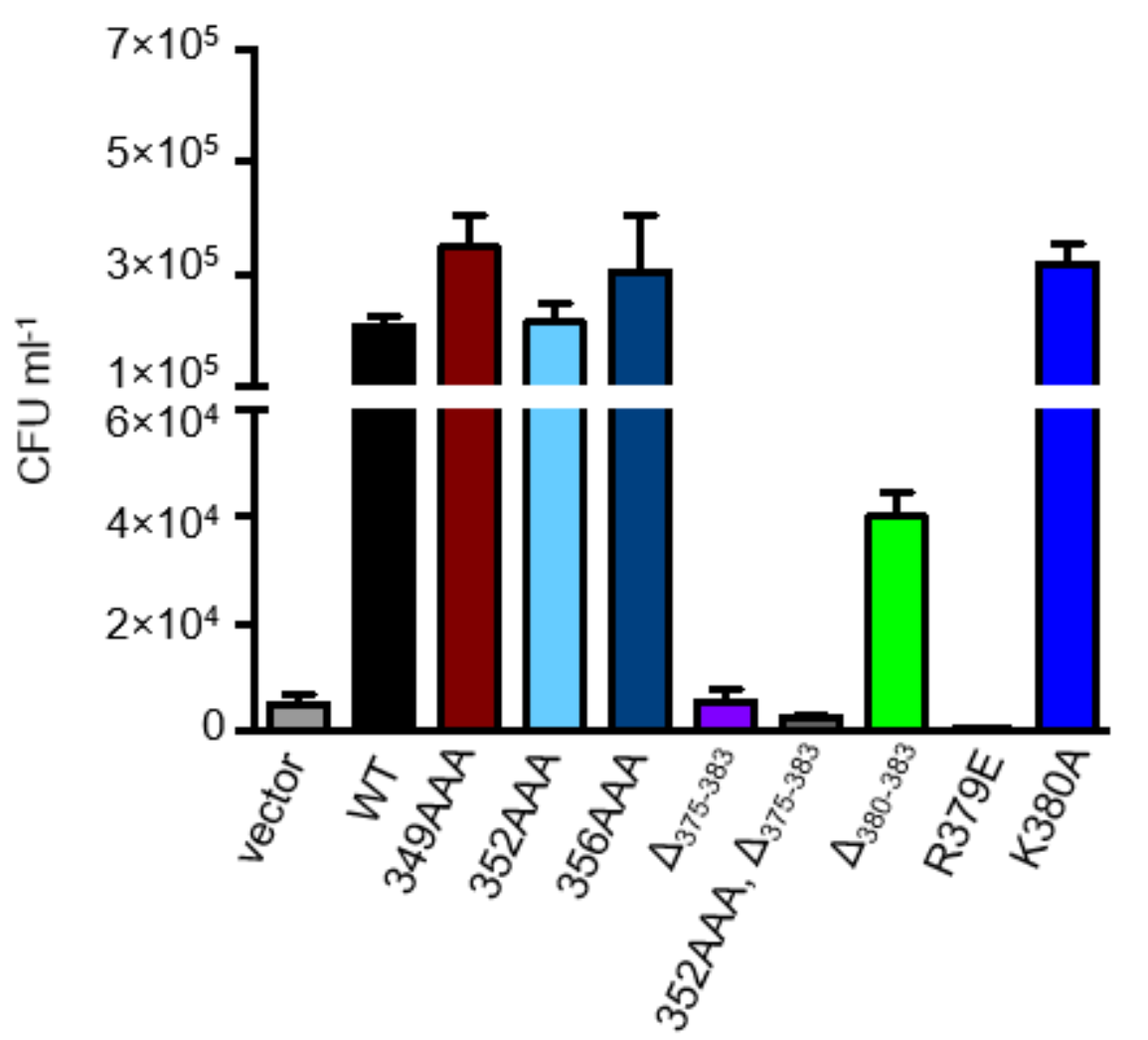


TABLE S1. E. coli strains and plasmids used in functional assays in vivo

\begin{tabular}{|c|c|c|}
\hline Strain or Plasmid & Genotype & $\begin{array}{l}\text { Source, reference } \\
\text { or Construction }^{\text {a }}\end{array}$ \\
\hline \multicolumn{3}{|l|}{$\underline{\text { Strains }}$} \\
\hline MG1655 & $L A M-r p h-1$ & {$[21]$} \\
\hline \multirow[t]{2}{*}{ BW27750 } & BW25113 DE(araFGH) & {$[20]$} \\
\hline & ( $\triangle a r a E p$ kan PCP18-araE) & $\mathrm{P} 1(\mathrm{BW} 27750) \mathrm{x}$ \\
\hline JC0390 & MG1655 (LaraEp kan PCP18-araE) & MG1655 \\
\hline MCZ84 & $\begin{array}{l}\text { ftsZ84(ts), leu-260::Tn10, [araD139], } \\
\text { A(argF-lac)169, LAM-, e14-, flhD5301, } \\
\text { 4fruK-yeiR)725(fruA), relA1, rpsL150(strR), } \\
\text { rbsR22, } \Delta(\text { fimB-fimE)632(::IS1), deoC1 }\end{array}$ & {$[34]$} \\
\hline \multicolumn{3}{|l|}{$\underline{\text { Plasmids }}$} \\
\hline pBAD24 & amp (expression vector) & {$[22]$} \\
\hline pBAD-FtsZ & amp $\mathrm{P}_{\text {ara }}:: f t s Z$ & {$[23]$} \\
\hline pBAD-FtsZ(P375G) & amp $\mathrm{P}_{\text {ara }}:: f t s Z(P 375 G)$ & This study \\
\hline pBAD-FtsZ(A376V) & amp $\mathrm{P}_{\text {ara }}:: f t s Z(A 376 V)$ & This study \\
\hline pBAD-FtsZ(F377A) & amp $\mathrm{P}_{\text {ara }}:: f t s Z(F 377 A)$ & This study \\
\hline pBAD-FtsZ(L378A) & amp $\mathrm{P}_{\text {ara }}:: f t s Z(L 378 A)$ & This study \\
\hline pBAD-FtsZ(R379E) & amp $\mathrm{P}_{\text {ara }}:: f t s Z(R 379 E)$ & This study \\
\hline pBAD-FtsZ(K380A) & amp $\mathrm{P}_{\text {ara }}:: f t s Z(K 380 A)$ & This study \\
\hline pBAD-FtsZ $\left(\Delta_{380-383}\right)$ & amp $\mathrm{P}_{\text {ara }}:: f t s Z\left(\Delta_{380-383}\right)$ & This study \\
\hline $\operatorname{pBAD}-\operatorname{FtsZ}\left(\Delta_{375-383}\right)$ & amp $\mathrm{P}_{\text {ara }}:: f t s Z\left(\Delta_{375-383}\right)$ & This study \\
\hline pBAD-FtsZ(349AAA) & amp $\mathrm{P}_{\text {ara }}:: f t s Z(349 A A A)$ & This study \\
\hline pBAD-FtsZ(352AAA) & amp $\mathrm{P}_{\text {ara }}:: f t s Z(352 A A A)$ & This study \\
\hline pBAD-FtsZ(356AAA) & amp $\mathrm{P}_{\text {ara }}:: f t s Z(356 A A A)$ & This study \\
\hline pBAD-FtsZ(352AAA, $\Delta_{375-383)}$ & amp $\mathrm{P}_{\text {ara }}:: f t s Z\left(352 A A A, \Delta_{375-383}\right)$ & This study \\
\hline pGfp-FtsZ & amp $\mathrm{P}_{a r a}:: g f p-f t s Z$ & {$[23]$} \\
\hline pGfp-FtsZ(P375G) & amp $\mathrm{P}_{\text {ara }}:: g f p-f t s Z(P 375 G)$ & This study \\
\hline pGfp-FtsZ(A376V) & amp $\mathrm{P}_{\text {ara }}:: g f p-f t s Z(A 376 V)$ & This study \\
\hline pGfp-FtsZ(F377A) & amp $\mathrm{P}_{\text {ara }}:: g f p-f t s Z(F 377 A)$ & This study \\
\hline pGfp-FtsZ(L378A) & amp $\mathrm{P}_{\text {ara }}:: g f p-f t s Z(L 378 A)$ & This study \\
\hline pGfp-FtsZ(R379E) & amp $\mathrm{P}_{\text {ara }}: g f p-f t s Z(R 379 E)$ & This study \\
\hline pGfp-FtsZ(K380A) & amp $\mathrm{P}_{\text {ara }}:: g f p-f t s Z(K 380 A)$ & This study \\
\hline pGfp-FtsZ $\left(\Delta_{380-383}\right)$ & amp $\mathrm{P}_{\text {ara }}:: g f p-f t s Z\left(\Delta_{380-383}\right)$ & This study \\
\hline pGfp-FtsZ $\left(\Delta_{375-383}\right)$ & amp $\mathrm{P}_{\text {ara }}:: g f p-f t s Z\left(\Delta_{375-383)}\right.$ & This study \\
\hline pGfp-FtsZ(349AAA) & amp $\mathrm{P}_{\text {ara }}:: g f p-f t s Z(349 A A A)$ & This study \\
\hline pGfp-FtsZ(352AAA) & amp $\mathrm{P}_{\text {ara }}:: g f p-f t s Z(352 A A A)$ & This study \\
\hline pGfp-FtsZ(356AAA) & amp $\mathrm{P}_{a r a}:: g f p-f t s Z(356 A A A)$ & This study \\
\hline
\end{tabular}


${ }^{a}$ Strain constructions by P1 transduction are described as the following: P1(donor) x recipient. 
FIGURE S1. Substitution of residues near the FtsZ C-terminus modulates the rate of degradation by ClpXP. Comparison of rates of degradation of FtsZ, FtsZ(L378A), FtsZ(F377A), FtsZ(A376V) and FtsZ(P375G) in the presence and absence of GTP from in vitro degradation reactions containing $10 \mu \mathrm{M}$ wild type or mutant fluorescent FtsZ and $1 \mu \mathrm{M}$ ClpXP. Data from 3 replicates are presented as mean $\pm \mathrm{SEM}$. 


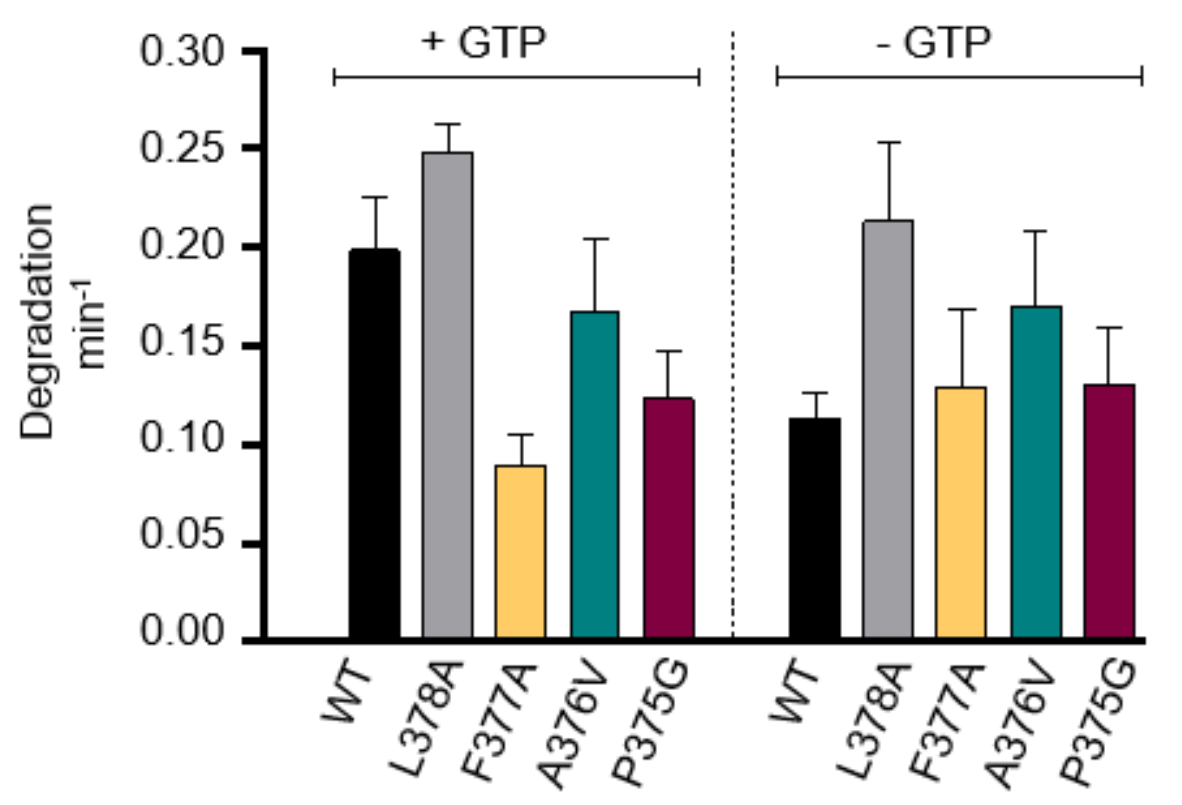


FIGURE S2. FtsZ mutant proteins with C-terminal mutations assemble into filaments. FtsZ mutant proteins $(5 \mu \mathrm{M})$ were incubated GTP, then visualized by negative staining and electron microscopy as described in Experimental Procedures (SI). Arrows point to the appearance of filament pairs or bundles in micrographs showing

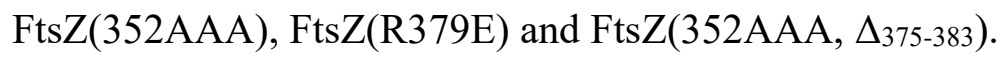



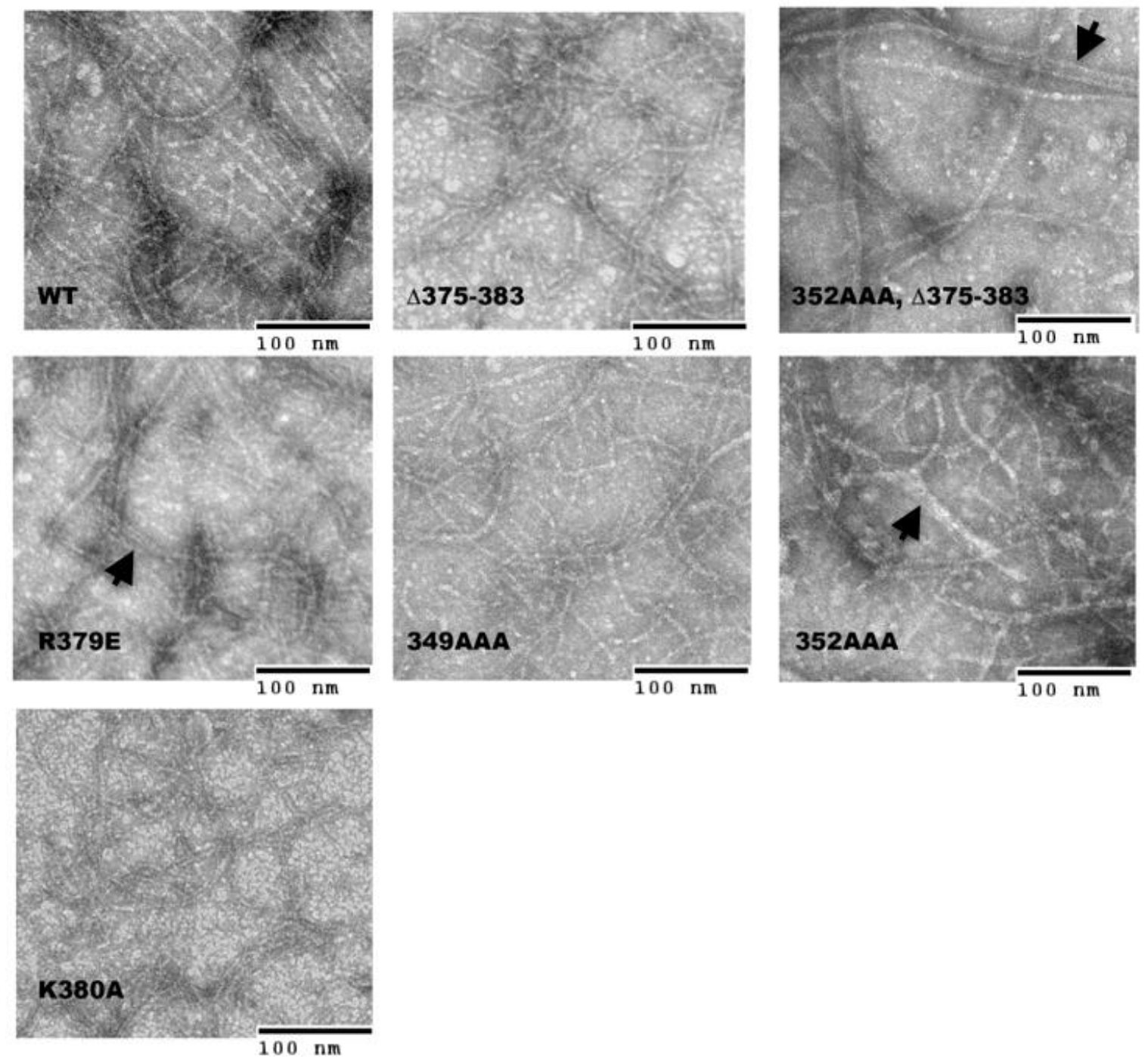
FIGURE S3. MinC does not inhibit degradation of GFP-ssrA by ClpXP. Degradation of GFP-ssrA $(0.5 \mu \mathrm{M})$ by ClpXP $(0.4 \mu \mathrm{M})$ was monitored as described in Experimental Procedures in the presence and absence of MinC $(5 \mu \mathrm{M})$ by measuring the decrease of fluorescence over time. 


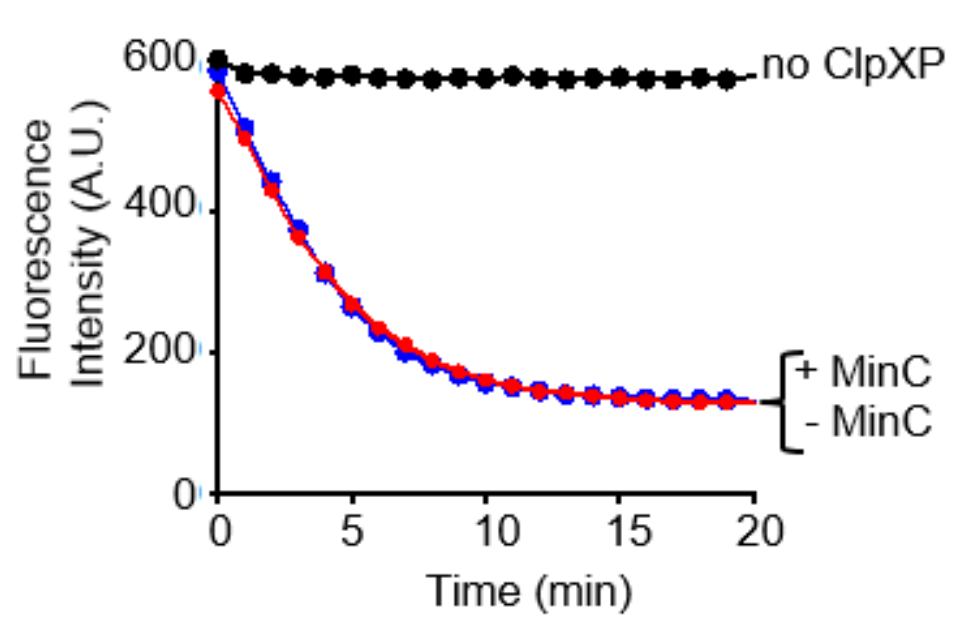


FIGURE S4. Mutations near the FtsZ C-terminal domain impair FtsZ function in vivo. A. FtsZ mutant proteins were tested for function in vivo by monitoring high temperature growth of ftsZ84 cells expressing FtsZ mutant proteins in a dilution spot plate assay under permissive $\left(30{ }^{\circ} \mathrm{C}\right)$ and restrictive $\left(42{ }^{\circ} \mathrm{C}\right)$ conditions. B. Z-ring localization of GFP-FtsZ mutant proteins was visualized by fluorescence microscopy in live cells (strain JC0390) undergoing division. Expression of GFP-FtsZ mutants proteins was induced by arabinose as described in Experimental Procedures (SI). Images are representative of at least 3 data sets. 

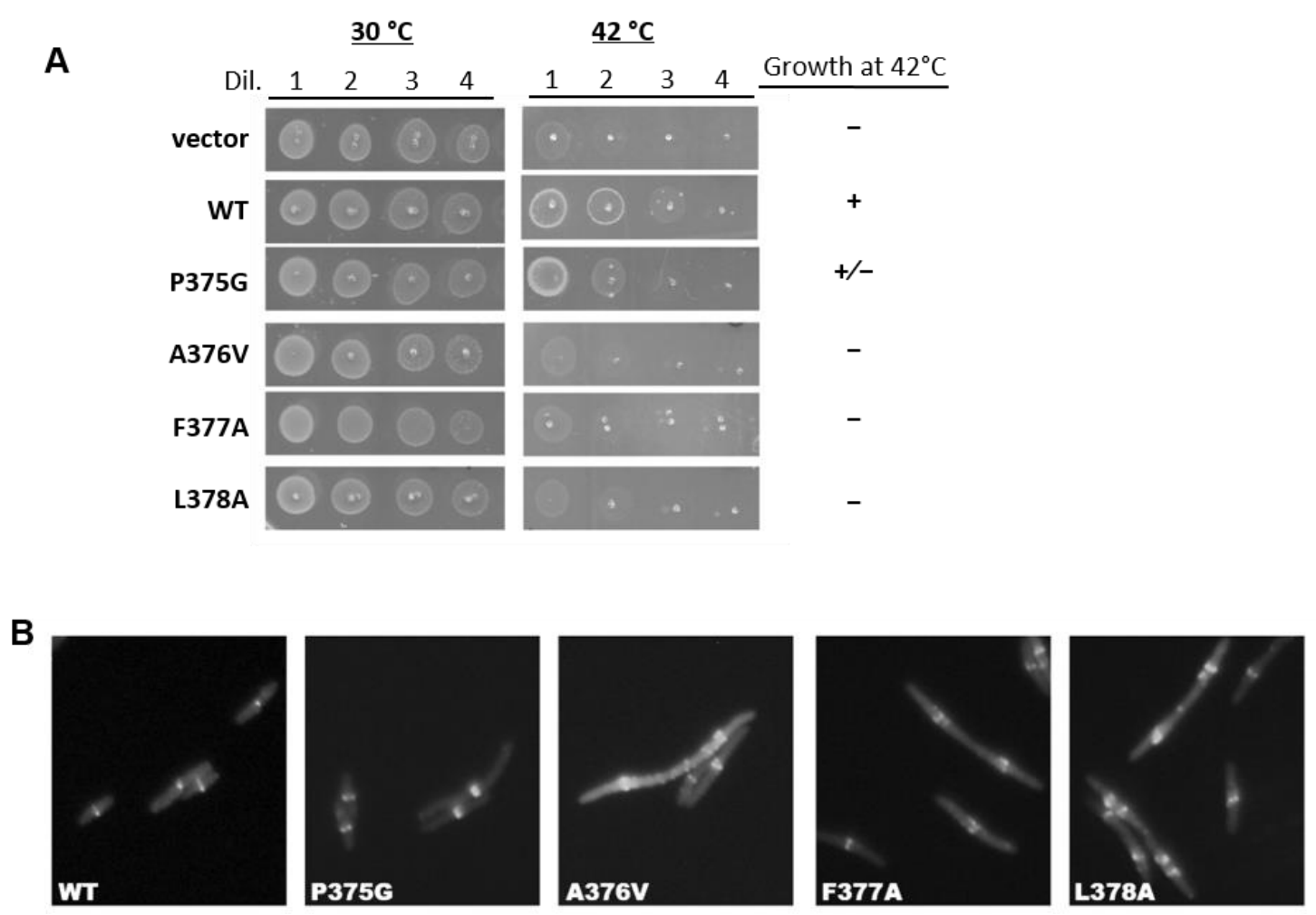
FIGURE S5. Expression of GFP-tagged FtsZ mutant proteins causes Z-ring defects. Z-ring localization of GFP-FtsZ wild type (WT) and mutant proteins was visualized by fluorescence microscopy (top panel) and DIC microscopy (bottom panel) in live cells (strain JC0390) undergoing division. Expression of GFP-FtsZ mutant proteins was induced by arabinose and cells were imaged as described in Experimental Procedures $(S I)$. 

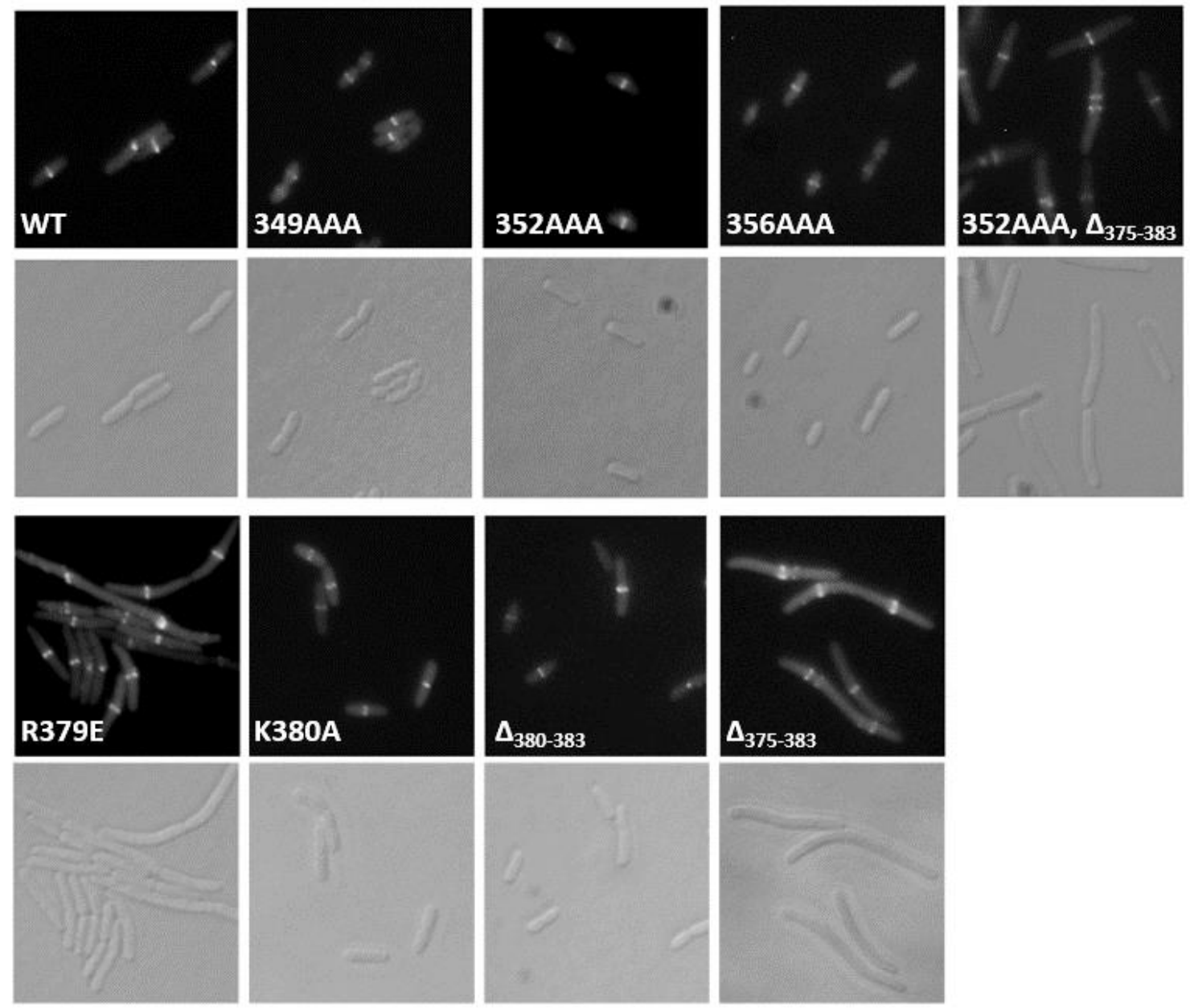


\section{Manuscript III}

\section{Publication status: Formatted for Protein Science}

Title: Tandem tags drive conformation-specific substrate processing of the cell division protein FtsZ by ClpXP in Escherichia coli

Authors: Marissa G. Viola ${ }^{\text {, }}{ }^{*}$ Jodi L. Camberg ${ }^{\mathrm{a}}$

\section{Author Affiliations:}

${ }^{a}$ Department of Cell and Molecular Biology, College of Environment and Life Sciences, University of Rhode Island, Kingston, RI, USA

*Corresponding author: Jodi L. Camberg, 120 Flagg Road, Kingston, RI, 02881; Tel: (401)874-4961; Email: cambergj@uri.edu 


\section{Abstract}

The ATP-dependent chaperone ClpX is a Clp/Hsp100 protein and member of the AAA+ (ATPases associated with diverse cellular activities) superfamily. In complex with ClpP, ClpXP recognizes and degrades over 100 diverse cellular substrates in Escherichia coli to regulate cellular processes. ClpXP comprises a hexameric $\mathrm{ClpX}$ ring that recognizes, unfolds, and translocates substrates to ClpP, a compartmentalized, barrelshaped tetradecamer and serine protease, for degradation. $\mathrm{ClpX}$ recognizes many substrates via adaptors for degradation, but also recognizes some proteins directly at the ClpX N-domain via specific recognition motifs, known as degrons. For several substrates that oligomerize, degradation by ClpXP is enhanced becasuse of an increase the local concentration of degrons. ClpXP degrades both unassembled and polymerized populations of the native Escherichia coli substrate and tubulin homolog FtsZ during cell division. FtsZ contains a globular polymerization domain (residues 1-316), a poorly conserved, disordered linker (residues 317-369), and a structured C-terminal region (residues 370-383). ClpX recognizes FtsZ at the disordered linker (residues 352-358, known as degron 2) and the extreme C-terminus (residues 375-383, known as degron 1). Purified FtsZ is a mixture of monomers and dimers, which complicates experiments designed to study recognition of monomeric FtsZ. To determine if both recognition sites (degrons 1 and 2) are utilized to degrade monomers, we engineered a chimeric protein containing Green fluorescent protein (Gfp) fused to the $67 \mathrm{C}$-terminal residues of FtsZ (Gfp- $\mathrm{Z}_{\mathrm{LC}}$ ), containing the linker and C-terminus (but not the polymerization domain), and monitored degradation by loss of fluorescence. ClpXP degrades Gfp- $Z_{L C}$ and Gfp- $Z_{\triangle \mathrm{DEG} 2}$, 
which contains alanine substitution mutations in place of the linker degron 2, but not Gfp- $Z_{\triangle D E G 1}$, which lacks the $C$-terminal degron 1 . These results suggest that only degron 1 is required to recognize an FtsZ monomer. However, degron 2 is important for degradation of FtsZ polymers. To determine if the ClpX N-domain is critical for the enhanced recognition of FtsZ polymers, we performed a peptide competition assay with a previously reported inhibitory peptide (XB peptide) containing the last 10 residues of the SspB adaptor, which binds to the ClpX N-domain. The SspB peptide contains sequence similarity with degron 1 and inhibits degradation of FtsZ. In the peptide competition assay, we determined that the $\mathrm{XB}$ peptide inhibits $\mathrm{Gfp}-\mathrm{Z}_{\mathrm{LC}}$ degradation twice as efficiently as polymers containing Gfp-FtsZ, suggesting that ClpX uses distinct modes of recognition for monomeric and polymeric FtsZ. These data suggest that ClpXP uses two sites for enhanced recognition of FtsZ polymers, whereas only one is used for recognition of an FtsZ monomer.

\section{Introduction}

ClpX from E. coli is a member of the AAA+ protein family. This family includes molecular machines that are powered by ATP hydrolysis and important for many cellular processes such as cell division, DNA replication, intracellular transport, and protein quality control ${ }^{1-3}$. Several of these AAA + family members are molecular chaperones that associate with proteases to catalyze coupled unfolding and degradation reactions in the cellular milieu. ClpX also associates with the ClpP protease to degrade proteins: characteristic pore loops at the ClpX C-terminus (known as "IGF" loops) make contacts 
with the ClpP tetradecamer surface and expand the pore in order to accept unfolded proteins for degradation ${ }^{16,17}$. Protein quality control is important for a variety of reasons: damaged or misfolded proteins can be removed from the crowded cellular environment to prevent aggregation that might damage or disrupt signaling pathways; misfolded proteins may also be remodeled into an alternate state or restored to the original molecular state for protein quality control ${ }^{1-3}$. Since degradation is irreversible, specific recognition regions with a high affinity for $\mathrm{ClpX}$, called degrons, are needed to ensure high fidelity recognition. Degrons may be present at either the N- or C-terminus of a native substrate ${ }^{4}$. Some substrates display multiple recognition motifs ("multivalent" recognition) to facilitate recognition by AAA + ATPases ${ }^{2}$. The bacterial chaperone-protease ClpXP can operate using a multivalent recognition strategy that enhances degradation of polymeric or oligomeric native substrates, including $\mathrm{MuA}$ and FtsZ, relative to the monomer conformations ${ }^{4-13}$. A single protomer of FtsZ contains two tandem degrons and FtsZ purifies as a mixture of monomers and dimers ${ }^{14}$. Since FtsZ polymers are degraded more efficiently than mixtures of FtsZ monomers and dimers, we hypothesize that ClpXP may use both degrons to recognize dimers or polymers, but may only require a single degron to degrade monomeric FtsZ ${ }^{6,7}$.

ClpX can operate independently as an unfoldase in vitro, but $\operatorname{clpX}$ is usually coexpressed with $c l p P$ in the same operon ${ }^{6,15}$. ClpX subunits contain two globular domains: a small $\mathrm{N}$-domain $(7 \mathrm{kDa})$ capable of dimerization also known as the zincbinding domain (ZBD), and the conserved $\mathrm{AAA}+$ domain which contains one large and one small subdomain ${ }^{17-19}$. Substrates are bound directly at the N-domain of ClpX or by pore loops in the $\mathrm{ClpX}$ axial pore where unfolding occurs and are then translocated into 
the ClpP chamber for degradation ${ }^{16,17,20-22}$. Nucleotides bind in the cleft between the large and small $\mathrm{AAA}+$ subdomains, and conformational changes occurring between the subdomains as a result of binding, unbinding, and nucleotide hydrolysis promote translocation of substrates into the ClpX axial channel ${ }^{17}$.

Adaptor proteins promote the stable binding and engagement of some ClpXP substrates and interact directly with the ClpX N-domain. SspB is a well-characterized adaptor protein that recognizes an 11-amino acid sequence known as an "ssrA-tag" 23,24. This 11-amino acid sequence is appended by transfer-messenger RNA (tmRNA) when the ribosome stalls during protein synthesis (known as trans-translation), to target the misfolded or truncated polypeptide for degradation and to release the ribosome from the mRNA. ClpX binds FtsZ directly without the requirement of an adaptor protein through two recognition motifs near the FtsZ C-terminus ${ }^{7}$. FtsZ is structurally homologous to the eukaryotic protein tubulin; both FtsZ and tubulin are essential for cell division ${ }^{25-27}$. Upon nucleotide binding, conformational changes at the surface of the polymerization domains of FtsZ allow monomers to self-associate and polymerize ${ }^{28}$. In vivo, ClpXP degrades approximately $15 \%$ of FtsZ subunits per cell cycle, and ClpXP utilizes a mechanism of divalent substrate recognition for a single FtsZ protomer and multivalent substrate recognition for an FtsZ polymer to regulate FtsZ degradation in vivo ${ }^{6,7}$.

In this study, we used model, chimeric proteins to serve as monomeric and polymeric FtsZ substrates. To investigate the contributions of each degron in recognition of monomeric or polymeric substrates, we mutagenized the regions in the C-terminus important for $\mathrm{ClpX}$ recognition and monitored degradation of purified proteins in vitro. We investigated how ClpX may engage polymers versus monomers using a peptide 
competition assay, as the peptide is known to compete with several native substrates for binding to the ClpX N-domain. We engineered model FtsZ substrates with alternative architecture, including shortening the region between the recognition motifs and swapping the positions of each degron to further understand how ClpX engages FtsZ. Our results suggest that although each degron contains a similar C-terminal motif, the relative position of each is important for discrimination between polymerized and monomeric substrates.

\section{Results}

ClpX requires the C-terminal degron to degrade $G f p-Z_{L C}$

Purified FtsZ exists as a mixture of monomers and dimers ${ }^{14}$. Unassembled FtsZ contains a mixture of substrate types - monomers contain two degrons, while dimers contain four degrons, and therefore may behave more similarly to polymerized FtsZ. To determine how ClpX targets FtsZ monomers distinctly from polymers via the linker and C-terminal degrons, we engineered a model monomeric substrate: Gfp fused to the linker and $\mathrm{C}$-terminal regions of FtsZ (the $\mathrm{C}$-terminal 67 amino acids), Gfp- $\mathrm{Z}_{\mathrm{LC}}$, to serve as a proxy for FtsZ monomers [Fig. (1A)]. The Gfp- $Z_{L C}$ chimera does not include the polymerization domain ${ }^{28}$. In the presence of ClpXP (but not ClpX alone), we observe

Gfp- $Z_{\mathrm{LC}}$ and Gfp-FtsZ degradation by loss of fluorescence ${ }^{14}$. To determine the relative contributions of each degron for monomer recognition by ClpXP, we mutagenized residues in the linker and $\mathrm{C}$-terminal degrons of Gfp- $\mathrm{Z}_{\mathrm{LC}}[\mathrm{Fig}$. (1A)] and monitored degradation of the mutagenized, monomeric substrates. ClpXP degrades the linkerimpaired mutant protein $G f p-Z_{\triangle \mathrm{DEG} 2}$ similarly to $\mathrm{Gfp}-\mathrm{Z}_{\mathrm{LC}}$, but not the C-terminal mutant 
protein Gfp- $Z_{\triangle D E G 1}$ [Fig. (1B)]. These results suggest that the C-terminal region is required for recognition of an FtsZ monomer, but the linker is dispensable.

Next, we aimed to determine if the monomeric FtsZ substrate is an N-domain dependent substrate. The XB peptide, which contains residues important for docking the $\mathrm{SspB}$ adaptor protein to $\mathrm{ClpX}$, inhibits degradation of ssrA-tagged substrates, unassembled FtsZ, polymerized FtsZ, and other known native substrates; the XB peptide also has sequence similarity to the C-terminus of FtsZ ${ }^{7,29,30}$. ClpXP degradation of Gfp$Z_{\mathrm{LC}}$ was significantly impaired in the presence of the XB peptide, by approximately $80 \%$ compared to without peptide, and degradation leveled off after 60 minutes [Fig. (1C)]. Since degron 1 is required for degradation of Gfp- $Z_{\mathrm{LC}}$, we incubated Gfp- $Z_{\triangle \mathrm{DEG} 2}$ with ClpXP in the presence of the XB peptide and monitored degradation by loss of fluorescence. Gfp- $Z_{\mathrm{LC}}$ and $\mathrm{Gfp}-\mathrm{Z}_{\triangle \mathrm{DEG} 2}$ are degraded equally well by ClpXP and are equally impaired for degradation in the presence of the XB peptide [Fig. (1C)].

To directly test if degradation of the monomeric Gfp- $Z_{\mathrm{LC}}$ requires $\mathrm{N}$-domain recognition by $\mathrm{ClpX}$, we incubated $\mathrm{Gfp}-\mathrm{Z}_{\mathrm{LC}}$ and $\mathrm{Gfp}-\mathrm{Z}_{\triangle \mathrm{DEG} 2}$ with $\mathrm{ClpX}(\Delta 61)$, a $\mathrm{ClpX}$ mutant protein lacking the first 61 amino acids (which define the ClpX N-domain) and ClpP, and monitored degradation by monitoring loss of fluorescence. Neither Gfp- $Z_{\mathrm{LC}}$ nor Gfp- $Z_{\triangle \mathrm{DEG} 2}$ were degraded by $\mathrm{ClpX}(\Delta \mathrm{N} 61) \mathrm{P}$ [Fig. (1D)]. Next, we incubated known Ndomain-independent substrate Gfp-ssrA with $\mathrm{ClpXP}$ and $\mathrm{ClpX}(\Delta \mathrm{N} 61) \mathrm{P}$. As expected, Gfp-ssrA was rapidly and thoroughly degraded by both ClpXP and ClpX( $\Delta$ N61)P [Fig. (1D)]. These results demonstrate that Gfp- $Z_{\mathrm{LC}}$ is an $\mathrm{N}$-domain dependent substrate and that loss of degron 2 does not further impair degradation. Since the C-terminal degron 1 is required for degradation of Gfp- $Z_{\mathrm{LC}}$ and is intact in Gfp- $Z_{\triangle \mathrm{DEG}}$, these results suggest 
that the C-terminal region of FtsZ may dock to the N-domain of ClpX.

\section{ClpX engages Fts $Z$ polymers via the linker degron 2}

Impairing both degrons 1 and 2 abolishes degradation of unassembled and polymerized FtsZ, while impairing either degron significantly reduces the degradation of FtsZ ${ }^{7,14}$. To determine if ClpX utilizes degron 2 for polymer recognition, we monitored degradation of either Gfp- $Z_{\mathrm{LC}}$ or polymers containing Gfp-FtsZ in the presence of increasing concentrations of the XB peptide. The XB peptide inhibits degradation of Gfp$\mathrm{Z}_{\mathrm{LC}}$ 2-fold more efficiently than polymers containing Gfp-FtsZ (Fig. 2). Since the XB peptide more efficiently impairs degradation of the Gfp- $Z_{\mathrm{LC}}$ monomer than polymers containing Gfp-FtsZ, the presence of degron 2 may affect the dynamic recognition of a polymerized FtsZ substrate by ClpX.

\section{Relative degron position and distance affect FtsZ polymer degradation}

Both FtsZ degrons 1 and 2 resemble the C-motif 2 sequence described for ClpX substrates ${ }^{4}$. To determine if the relative position of each degron is important for recognition by $\mathrm{ClpX}$, we constructed mutant proteins with swapped degron sequences [Fig. (3A)]. In the presence of GTP, both Gfp-FtsZ and Gfp-FtsZ $Z_{\text {SWAP1 }}$ were degraded equally well by ClpXP [Fig. (3B)], but in the absence of GTP, unassembled GfpFtsZ $_{\text {SWAP1 }}$ was degraded significantly more than unassembled Gfp-FtsZ [Fig. (3C)]. These results suggest that although the degron sequences contain similarities, the residues flanking the degrons may also affect recognition.

Next, we constructed a mutant protein without the 16-amino acid sequence 
between the swapped degrons [Gfp-FtsZ $Z_{\mathrm{SWAP} 2}$, Fig. (3A)], and monitored the effect of degradation in the presence and absence of GTP by ClpXP. In the presence of GTP, degradation of Gfp-FtsZswAP2 polymers by ClpXP was significantly reduced [Fig. (3D)]. Since swapping the degrons did not impair degradation of an FtsZ substrate, but subsequently removing the space between them did, this result suggests that the relative positioning of each may be important for docking the N-domain of ClpX. In the absence of GTP, degradation of unassembled Gfp-FtsZ $Z_{\text {SWAP2 }}$ by ClpXP was also significantly reduced [Fig. (3E)]. Since both unassembled and polymeric Gfp-FtsZ $Z_{\text {SwAP1 }}$ are degraded better than unassembled and polymeric Gfp-FtsZ $Z_{\text {SWAP2, }}$, the distance between the degrons may be important.

Multimerization is important for interactions with the FtsZ C-terminus in vivo

Although ClpXP degrades polymerized FtsZ more efficiently, ClpXP recognizes and degrades unassembled FtsZ protomers and does not require polymerization for engagement ${ }^{7,31}$. Other cell division regulators recognize FtsZ via the C-terminus at single amino acid residues, but unlike $\mathrm{ClpX}$, some proteins interact with the polymerization domain of FtsZ in addition to the C-terminal region ${ }^{32-34}$.

In the early septal phase of cell division in E. coli, FtsZ forms a network of overlapping polymers ${ }^{34,35}$. When expressed in vivo, Gfp-FtsZ localizes at midcell during $\log$ phase ${ }^{36}$. To determine if multimerization is required for recruitment of Gfp- $Z_{\mathrm{LC}}$ to the septal ring, we overexpressed Gfp- $Z_{\mathrm{LC}}$ with an arabinose-inducible promoter at increasingly high concentrations in vivo and examined cell physiology during log phase. Gfp- $Z_{\mathrm{LC}}$ did not localize at midcell at arabinose concentrations 20 -fold higher than the 
minimum concentration required to visualize Gfp-FtsZ rings in wild type cells (Fig. 4). These results suggest that recruitment of FtsZ via the C-terminus requires multimerization via the FtsZ polymerization domain during septal ring formation in vivo.

\section{Discussion}

In our model for FtsZ substrate recognition by $\mathrm{ClpX}$, ClpX requires degron 1 to degrade the monomeric substrate Gfp- $Z_{\mathrm{LC}}$, but degron 2 is dispensable [Fig. (5A, 5B)]. The first step in the degradation pathway of a target substrate is initial substrate binding, followed by substrate engagement by the $\mathrm{ClpX}$ pore, and subsequent translocation into the ClpP chamber for degradation. In our study, impaired degradation does not differentiate between impaired binding or unfolding. Further studies could determine if Gfp- $Z_{\triangle D E G 1}$ is defective for direct binding to ClpX; if binding is not impaired, then degron 2 may be engaged in the monomeric substrate, but not sufficient to promote processive degradation. Substrate engagement with pore loops in the upper and lower regions of the ClpX channel are important for processivity, and the detection of binding for a substrate that is not degraded would demonstrate that the substrate is alternatively engaged ${ }^{37}$.

We determined that the XB peptide signficantly impairs degradation of Gfp- $Z_{\mathrm{LC}}$. Therefore, we propose that monomers are $\mathrm{N}$-domain dependent substrates [Fig. (5A, 5B)]. We also determined that the $\mathrm{XB}$ peptide inhibits $\mathrm{Gfp}-\mathrm{Z}_{\mathrm{LC}}$ twice as efficiently as polymers containing Gfp-FtsZ. We further propose that ClpX recognizes degron 2 in an $\mathrm{N}$-domain independent manner, while degron 1 likely docks the N-domain [Fig. (5C)]. We also determined that relative degron position does not enhance degradation of 
polymerized FtsZ by ClpXP, but that the space between degrons does. Our results are consistent with the model for MuA, in which the secondary degron acts as an enhancer to promote multivalent substrate recognition of the MuA tetramer ${ }^{13}$. Further studies could determine the theoretical $\mathrm{K}_{\mathrm{D}}$ of monomeric and polymeric FtsZ substrates using the realtime degradation assays in this study.

Gfp- $Z_{\mathrm{LC}}$ does not localize to midcell under the conditions tested in this study [Fig. (4A)], suggesting that the FtsZ polymerization domain is required for multimerization and potential multivalent substrate recognition in vivo. Impairing degron 2 in Gfp-FtsZ does not perturb Z-ring formation, localization, or significantly affect overall cell length during log phase, but overexpressing Gfp-FtsZ with an impaired degron 1 causes cell filamentation and several Z-rings per cell length ${ }^{7,14}$. These results are not surprising, since many cell division regulators bind FtsZ via the C-terminus to modulate FtsZ polymerization dynamics in vivo, whereas $\mathrm{ClpX}$, which is not essential for cell division in E. coli, is the only known protein to recognize FtsZ in the linker region ${ }^{7,32}$. Since degron 2 is intact in degron 1-impaired Gfp-FtsZ, however, it is interesting that degron 2, which we propose here acts as an enhancer for recognition during multimerization, is not sufficient to promote proper Z-ring localization and cell length during division ${ }^{7}$. This further implicates the importance of the enhancer degron 2 when degron 1 is also intact, and therefore the relative contributions of each degron may be important for directing multivalent recognition in vivo.

\section{Conclusions}

1. Degron 1 is required for degradation of a monomer by ClpXP, and likely docks on the N-domain [Fig. (5A, 5B)]. 
2. The XB peptide inhibits degradation of Gfp- $\mathrm{Z}_{\mathrm{LC}}$ and $\mathrm{ClpX}(\Delta \mathrm{N} 61) \mathrm{P}$ does not degrade Gfp-Z $Z_{\text {LC }}$, suggesting that monomers are N-domain dependent ClpX substrates [Fig. $(5 \mathrm{~A}, 5 \mathrm{~B})]$

3. The XB peptide inhibits degradation of Gfp- $Z_{\mathrm{LC}}$ twice as efficiently as polymerized Gfp-FtsZ:FtsZ (1:1), indicating that ClpX engages each substrate differently; degron 2 may facilitate polymer recognition independent of the N-domain [Fig. (5C)].

4. Relative degron position does not enhance degradation of polymerized FtsZ by ClpXP; however, the distance between degrons is important; degron 2 access may be important in a polymer [Fig. (5C)].

\section{Materials and Methods}

Proteins, plasmids, and peptides

Gfp- $Z_{\mathrm{LC}}$ and Gfp-FtsZ were cloned into plasmids as described in Viola et al. 2017, and all Gfp-Z $Z_{\mathrm{LC}}$, Gfp-FtsZ, and FtsZ mutant proteins were constructed by sitedirected mutagenesis of plasmids using the QuikChange II XL Site-Directed Mutagenesis Kit (Agilent). The pBad-Gfp- $Z_{\mathrm{LC}}$ was constructed by cloning Gfp- $Z_{\mathrm{LC}}$ into the NheI and HindIII sites on the arabinose inducible vector, pBad24 ${ }^{38}$. The pBad-Gfp-FtsZ plasmid was constructed as described ${ }^{39}$. The C-terminal SspB peptide ("XB peptide") sequence NH2-RGGRPALRVVK-COOH (as described in Camberg et al. 2014) was custom produced and purchased from Thermo Fisher Scientific.

Expression and purification of proteins 
All Gfp-Z $Z_{\mathrm{LC}}, \mathrm{Gfp}-\mathrm{FtsZ}$, and FtsZ wild type proteins were expressed in E. coli BL21 [B F ${ }^{-} d c m$ ompT hsdS clpP $\left(\mathrm{r}_{\mathrm{B}}{ }^{-} \mathrm{m}_{\mathrm{B}}{ }^{-}\right)$gal $\left.\lambda(\mathrm{DE} 3)\right]$, which was constructed for this study by deleting the $c l p P$ gene by P1 transduction from BL21 $\lambda(\mathrm{DE} 3)$ (Agilent), and purified as described (Table 1) ${ }^{14}$. Gfp-ssrA was expressed and purified as described ${ }^{40}$. $\mathrm{ClpX}$ and ClpP were expressed and purified as described ${ }^{41,42}$. Protein concentrations are reported as Gfp-Z $Z_{\mathrm{LC}}$ monomers, Gfp-ssrA monomers, Gfp-FtsZ or FtsZ monomers, ClpX hexamers, and ClpP tetradecamers.

\section{Degradation assays}

To monitor degradation by loss of fluorescence, Gfp-FtsZ and Gfp- $Z_{\mathrm{LC}}$ were degraded by ClpXP in buffer containing $20 \mathrm{mM}$ HEPES pH 7.0, $150 \mathrm{mM} \mathrm{KCl}$, and 10 $\mathrm{mM} \mathrm{MgCl} 2$ with $5 \mathrm{mM}$ ATP, $0.005 \%$ Triton X-100, and 2mM GTP, where indicated, and acetate kinase $\left(25 \mu \mathrm{g} \mathrm{ml} \mathrm{m}^{-1}\right)$ and acetyl phosphate $(15 \mathrm{mM})$ (bifunctional ATP/GTP regenerating system), where indicated. Importantly, although Gfp(uv) has been reported to dimerize at high concentrations, we used Gfp-fusion proteins well below the dimerization conditions ${ }^{43}$. Where indicated, Gfp-FtsZ polymers were homogeneous populations or a mixture of 1:1, Gfp-FtsZ:FtsZ. Fluorescence was monitored in a 96-well plate with a Cary Eclipse Spectrophotometer (Agilent) at an excitation wavelength of 395 $\mathrm{nm}$ and emission of $510 \mathrm{~nm}$. The background signal from buffer was subtracted from each data set and normalized to report the percentage of arbitrary fluorescence units lost.

To determine the rate of degradation, Gfp fluorescence was recorded as a fraction of the initial and the arbitrary units of fluorescence degraded per minute (A.U. min $^{-1}$ ) was determined. For the peptide competition assays with the XB peptide and FtsZ substrate, 
the initial rates for degradation in the absence of $\mathrm{XB}$ peptide were averaged, and individual degradation rates in the presence of increasing concentrations of XB peptide were divided by the averaged, initial degradation rate. The curve was fit using actual values, but plotted manually on a log scale using GraphPad Prism (version 6.0b).

\section{Microscopy}

The MG1655-derived strain (JC0390 from Viola et al. 2017) was transformed with $\mathrm{pBad}-\mathrm{Gfp}-\mathrm{Z}_{\mathrm{LC}}$ or $\mathrm{pBad}-\mathrm{FtsZ}$, and overnight cultures were diluted and grown to logarithmic conditions and induced with different concentrations of arabinose as described ${ }^{14}$. Images were collected with a Zeiss LSM 700 confocal fluorescence microscope and images were captured on an AxioCam digital camera with ZEN 2012 software.

\section{Acknowledgements}

We thank Christopher J. LaBreck, Joseph Conti, Eric DiBiasio, Cathy Trebino, and Shannon May for helpful discussions. We would also like to thank Janet Atoyan for sequencing assistance and coordination using the microscope; both sequencing and microscopy were performed at the Rhode Island Genomics and Sequencing Center, which is supported in part by the National Science Foundation under EPSCoR Grants Nos. 0554548 \& EPS-1004057.

\section{References}

1. Sauer, R. T. \& Baker, T. a. AAA+ proteases: ATP-fueled machines of protein destruction. Annu. Rev. Biochem. 80, 587-612 (2011). 
2. Baker, T. a. \& Sauer, R. T. ATP-dependent proteases of bacteria: recognition logic and operating principles. Trends Biochem. Sci. 31, 647-653 (2006).

3. Snider, J., Thibault, G. \& Houry, W. a. The AAA + superfamily of functionally diverse proteins. Genome Biol. 9, 216 (2008).

4. Flynn, J. M., Neher, S. B., Kim, Y. I., Sauer, R. T. \& Baker, T. a. Proteomic discovery of cellular substrates of the ClpXP protease reveals five classes of ClpXrecognition signals. Mol. Cell 11, 671-683 (2003).

5. Neher, S. B. et al. Proteomic Profiling of ClpXP Substrates after DNA Damage Reveals Extensive Instability within SOS Regulon. Mol. Cell 22, 193-204 (2006).

6. Camberg, J. L., Hoskins, J. R. \& Wickner, S. ClpXP protease degrades the cytoskeletal protein, FtsZ, and modulates FtsZ polymer dynamics. Proc. Natl. Acad. Sci. U. S. A. 106, 10614-10619 (2009).

7. Camberg, J. L., Viola, M. G., Rea, L., Hoskins, J. R. \& Wickner, S. Location of dual sites in E. coli FtsZ important for degradation by ClpXP; one at the Cterminus and one in the disordered linker. PLoS One 9, 12-14 (2014).

8. Conti, J., Viola, M. G. \& Camberg, J. L. The bacterial cell division regulators MinD and MinC form polymers in the presence of nucleotide. FEBS Lett. 589, 201-206 (2015).

9. Stephani, K., Weichart, D. \& Hengge, R. Dynamic control of Dps protein levels by ClpXP and ClpAP proteases in Escherichia coli. Mol. Microbiol. 49, 1605-1614 (2003).

10. Abdelhakim, A. H., Oakes, E. C., Sauer, R. T. \& Baker, T. A. Unique Contacts Direct High-Priority Recognition of the Tetrameric $\mathrm{Mu}$ Transposase-DNA 
Complex by the AAA+ Unfoldase ClpX. Mol. Cell 30, 39-50 (2008).

11. Abdelhakim, A. H., Sauer, R. T. \& Baker, T. a. The AAA+ ClpX machine unfolds a keystone subunit to remodel the Mu transpososome. Proc. Natl. Acad. Sci. U. S. A. 107, 2437-2442 (2010).

12. Adams, D. W., Wu, L. J. \& Errington, J. Cell cycle regulation by the bacterial nucleoid. Curr. Opin. Microbiol. 22, 94-101 (2014).

13. Ling, L., Montano, S. P., Sauer, R. T., Rice, P. A. \& Baker, T. A. Deciphering the roles of the multi-component recognition signals by the AAA+ unfoldase, ClpX. $J$. Mol. Biol. 427, 2966-2982 (2015).

14. Viola, M. G., Labreck, C. J., Conti, J. \& Camberg, J. L. Proteolysis-dependent remodeling of the tubulin homolog FtsZ at the division septum in Escherichia coli. PLoS One 12, 1-23 (2017).

15. Yoo, S. J., Seol, J. H., Kang, M. S., Ha, D. B. \& Chung, C. H. clpX Encoding an Alternative ATP-Binding Subunit of Protease Ti (Clp) Can Be Expressed Independently from clpP in Escherichia coli. Biochem. Biophys. Res. Commun. 203, 798-804 (1994).

16. Kim, Y.-I. et al. Molecular determinants of complex formation between Clp/Hsp100 ATPases and the ClpP peptidase. Nat Struct Mol Biol 8, 230-233 (2001).

17. Baker, T. A. \& Sauer, R. T. ClpXP, an ATP-powered unfolding and proteindegradation machine. Biochim. Biophys. Acta - Mol. Cell Res. 1823, 15-28 (2012).

18. Gottesman, S. Proteolysis in Bacterial Regulatory Circuits. Annu. Rev. Cell Dev. Biol. 19, 565-587 (2003). 
19. Wojtyra, U. A., Thibault, G., Tuite, A. \& Houry, W. A. The N-terminal zinc binding domain of $\mathrm{ClpX}$ is a dimerization domain that modulates the chaperone function. J. Biol. Chem. 278, 48981-48990 (2003).

20. Glynn, S. E., Martin, A., Nager, A. R., Baker, T. a. \& Sauer, R. T. Structures of Asymmetric ClpX Hexamers Reveal Nucleotide-Dependent Motions in a AAA+ Protein-Unfolding Machine. Cell 139, 744-756 (2009).

21. Wang, J., Hartling, J. a \& Flanagan, J. M. The Structure of ClpP at $2.3 \AA$ Resolution Suggests a Model for ATP-Dependent Proteolysis. Cell 91, 447-456 (1997).

22. Baker T. and Sauer, R. ClpX, an ATP-powered unfolding and protein-degradation machine. 1823, 15-28 (2013).

23. Gottesman, S., Roche, E., Zhou, Y. N. \& Sauer, R. T. The ClpXP and ClpAP proteases degrade proteins with carboxy-terminal peptide tails added by the SsrAtagging system. Genes Dev. 12, 1338-1347 (1998).

24. Levchenko, I., Seidel, M., Sauer, R. T. \& Baker, T. A. A Specificity-Enhancing Factor for the ClpXP Degradation Machine. Science (80-. ). 289, 2354 LP-2356 (2000).

25. Erickson, H. P. \& Stoffler, D. Protofilaments and rings, two conformations of the tubulin family conserved from bacterial FtsZ to alpha/beta and gamma tubulin. $J$. Cell Biol. 135, 5-8 (1996).

26. Erickson, H. P., Taylor, D. W., Taylor, K. a \& Bramhill, D. Bacterial cell division protein FtsZ assembles into protofilament sheets and minirings, structural homologs of tubulin polymers. Proc. Natl. Acad. Sci. U. S. A. 93, 519-523 (1996). 
27. Nogales, E., Downing, K. H., Amos, L. a., Löwe, J. \& Lowe, J. Tubulin and FtsZ form a distinct family of GTPases. Nat. Struct. Biol. 5, 451-458 (1998).

28. Oliva, M. A., Cordell, S. C. \& Lowe, J. Structural insights into FtsZ protofilament formation. Nat Struct Mol Biol 11, 1243-1250 (2004).

29. Park, E. Y. et al. Structural Basis of SspB-tail Recognition by the Zinc Binding Domain of ClpX. J. Mol. Biol. 367, 514-526 (2007).

30. Wah, D. A. et al. Flexible linkers leash the substrate binding domain of SspB to a peptide module that stabilizes delivery complexes with the AAA+ClpXP protease. Mol. Cell 12, 355-363 (2003).

31. Camberg, J. L., Hoskins, J. R. \& Wickner, S. ClpXP protease degrades the cytoskeletal protein, FtsZ, and modulates FtsZ polymer dynamics. Proc Natl Acad Sci U S A 106, 10614-10619 (2009).

32. Ma, X. \& Margolin, W. Genetic and functional analyses of the conserved Cterminal core domain of Escherichia coli FtsZ. J. Bacteriol. 181, 7531-7544 (1999).

33. Du, S. \& Lutkenhaus, J. SlmA Antagonism of FtsZ Assembly Employs a Twopronged Mechanism like MinCD. PLoS Genet. 10, (2014).

34. Ortiz, C., Natale, P., Cueto, L. \& Vicente, M. The keepers of the ring: Regulators of FtsZ assembly. FEMS Microbiol. Rev. 40, 57-67 (2015).

35. Erickson, H. P., Anderson, D. E. \& Osawa, M. FtsZ in bacterial cytokinesis: cytoskeleton and force generator all in one. Microbiol. Mol. Biol. Rev. 74, 504-528 (2010).

36. Hale, C. A. \& De Boer, P. A. J. Recruitment of ZipA to the septal ring of 
Escherichia coli is dependent on FtsZ and independent of FtsA. J. Bacteriol. 181, $167-176$ (1999).

37. Martin, A., Baker, T. A. \& Sauer, R. T. Pore loops of the AAA + ClpX machine grip substrates to drive translocation and unfolding. Nat Struct Mol Biol 15, 1147-1151 (2008).

38. Guzman, L. M., Belin, D., Carson, M. J. \& Beckwith, J. Tight regulation , modulation, and high-level expression by vectors containing the arabinose These include: Tight Regulation, Modulation, and High-Level Expression by Vectors Containing the Arabinose P BAD Promoter. 177, (1995).

39. Camberg, J. L., Hoskins, J. R. \& Wickner, S. The Interplay of ClpXP with the cell division machinery in Escherichia coli. J. Bacteriol. 193, 1911-1918 (2011).

40. Yakhnin, A. V, Vinokurov, L. M., Surin, A. K. \& Alakhov, Y. B. Green Fluorescent Protein Purification by Organic Extraction. Protein Expr. Purif. 14, 382-386 (1998).

41. Maurizi, M. R., Thompson, M. W., Singh, S. K. \& Kim, S.-H. [23] Endopeptidase Clp: ATP-dependent Clp protease from Escherichia coli. Methods Enzymol. 244, 314-331 (1994).

42. Grimaud, R., Kessel, M., Beuron, F., Steven, A. C. \& Maurizi, M. R. Enzymatic and Structural Similarities between theEscherichia coli ATP-dependent Proteases, ClpXP and ClpAP. J. Biol. Chem. 273, 12476-12481 (1998).

43. Prendergast, F. \& Mann, K. Chemical and physical properties of aequorin and the green fluorescent protein isolated from Aequorea forskålea. Biochemistry 22, $3448-53$ (1978). 
Figure 1. Degradation of a synthetic, monomeric FtsZ substrate by ClpXP. (A) Schematic for synthetic monomer FtsZ substrate, Gfp-Z $Z_{\mathrm{LC}}$ : fluorescent protein Gfp is fused to the C-terminal 67 amino acids of FtsZ comprising the linker region (residues 317-369, first white box) and the extreme C-terminal region (residues 370-383, second white box) of FtsZ. Either the extreme C-terminus is removed (Gfp- $\left.Z_{\triangle \mathrm{DEG1}}\right)$, or the recognized linker region is impaired with alanine mutagenesis $\left(\mathrm{Gfp}-\mathrm{Z}_{\triangle \mathrm{DEG} 2}\right)$. (B) Gfp- $\mathrm{Z}_{\mathrm{LC}}$ (3 $\mu \mathrm{M})$ (white circles), Gfp- $Z_{\Delta \mathrm{DEG} 1}(3 \mu \mathrm{M})$ (red circles), or Gfp- $Z_{\triangle \mathrm{DEG} 2}(3 \mu \mathrm{M})$ (gold circles) degradation was monitored by loss of fluorescence with time in the presence of ClpXP $(1 \mu \mathrm{M})$, ATP $(5 \mathrm{mM})$, and a regenerating system as described. Curves shown are representative of at least three replicates. (C) Gfp-Z $\mathrm{Z}_{\mathrm{LC}}(3 \mu \mathrm{M})$ (white circles) or Gfp$\mathrm{Z}_{\triangle \mathrm{DEG} 2}(3 \mu \mathrm{M})$ (gold diamonds) degradation was monitored by loss of fluorescence with time in the presence of ClpXP $(1 \mu \mathrm{M})$, ATP $(5 \mathrm{mM})$, and a regenerating system as described. Gfp- $Z_{\mathrm{LC}}(3 \mu \mathrm{M})$ (black circles) or Gfp- $Z_{\Delta \mathrm{DEG} 2}(3 \mu \mathrm{M})$ (red circles) degradation was monitored by loss of fluorescence with time in the presence of the XB peptide (160 $\mathrm{mM}), \mathrm{ClpXP}(1 \mu \mathrm{M}), \mathrm{ATP}(5 \mathrm{mM})$, and a regenerating system as described. Reaction buffer was monitored over time for reference (grey circles). Curves shown are representative of at least three replicates. (D) Gfp-ssrA $(1 \mu \mathrm{M})$ degradation was monitored by loss of fluorescence with time in the presence of ClpXP $(1 \mu \mathrm{M})$ (black circles) or $\mathrm{ClpX}(\Delta \mathrm{N} 61) \mathrm{P}(1 \mu \mathrm{M})$ (grey circles), ATP $(5 \mathrm{mM})$, and a regenerating system as described. Gfp- $Z_{\mathrm{LC}}(3 \mu \mathrm{M})$ (white circles) or $\operatorname{Gfp}_{-Z_{\Delta E G 2}}(3 \mu \mathrm{M})$ (red circles) degradation was monitored by loss of fluorescence with time in the presence of $\mathrm{ClpX}(\Delta \mathrm{N} 61) \mathrm{P}(1 \mu \mathrm{M})$, ATP $(5 \mathrm{mM})$, and a regenerating system as described. Curves shown are representative of at least three replicates. 
A

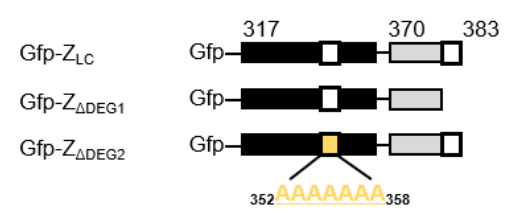

C

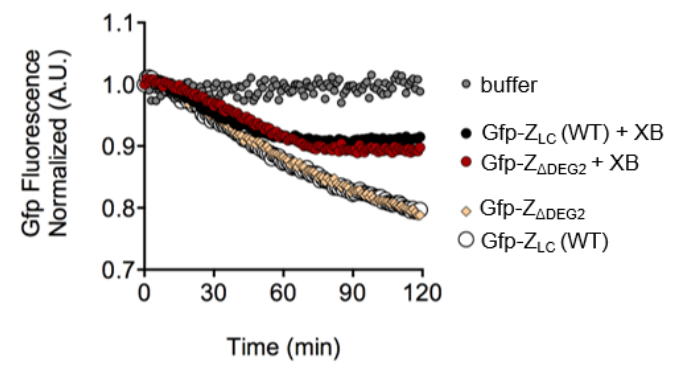

B

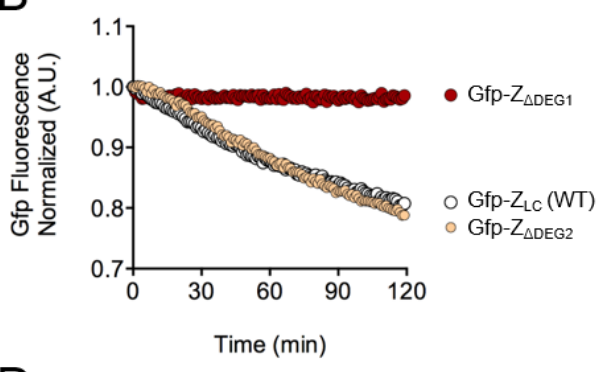

D

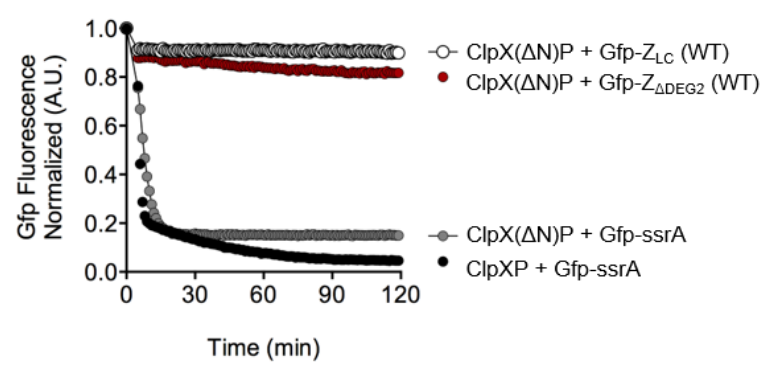


Figure 2. ClpX engagement of monomeric and polymeric substrates. Gfp-FtsZ (3 $\mu \mathrm{M})$ and FtsZ $(3 \mu \mathrm{M})$ were precincubated with GTP (2 mM), and degradation by ClpXP $(1 \mu \mathrm{M})$ with ATP $(5 \mathrm{mM})$ and a regenerating system was monitored with time as described in the presence of increasing concentrations of XB peptide $(0,5,10,20,40,80$ $\mu \mathrm{M})$. Gfp-Z $\mathrm{LC}_{\mathrm{L}}(6 \mu \mathrm{M})$ degradation by $\operatorname{ClpXP}(1 \mu \mathrm{M})$ with $\mathrm{ATP}(5 \mathrm{mM})$ and a regenerating system was monitored with time as described in the presence of increasing concentrations of $\mathrm{XB}$ peptide $(0,5,10,20,40,80 \mu \mathrm{M})$. Degradation rates were determined as described, and plotted as a fraction of the initial rate of degradation in the absence of XB peptide as shown. 


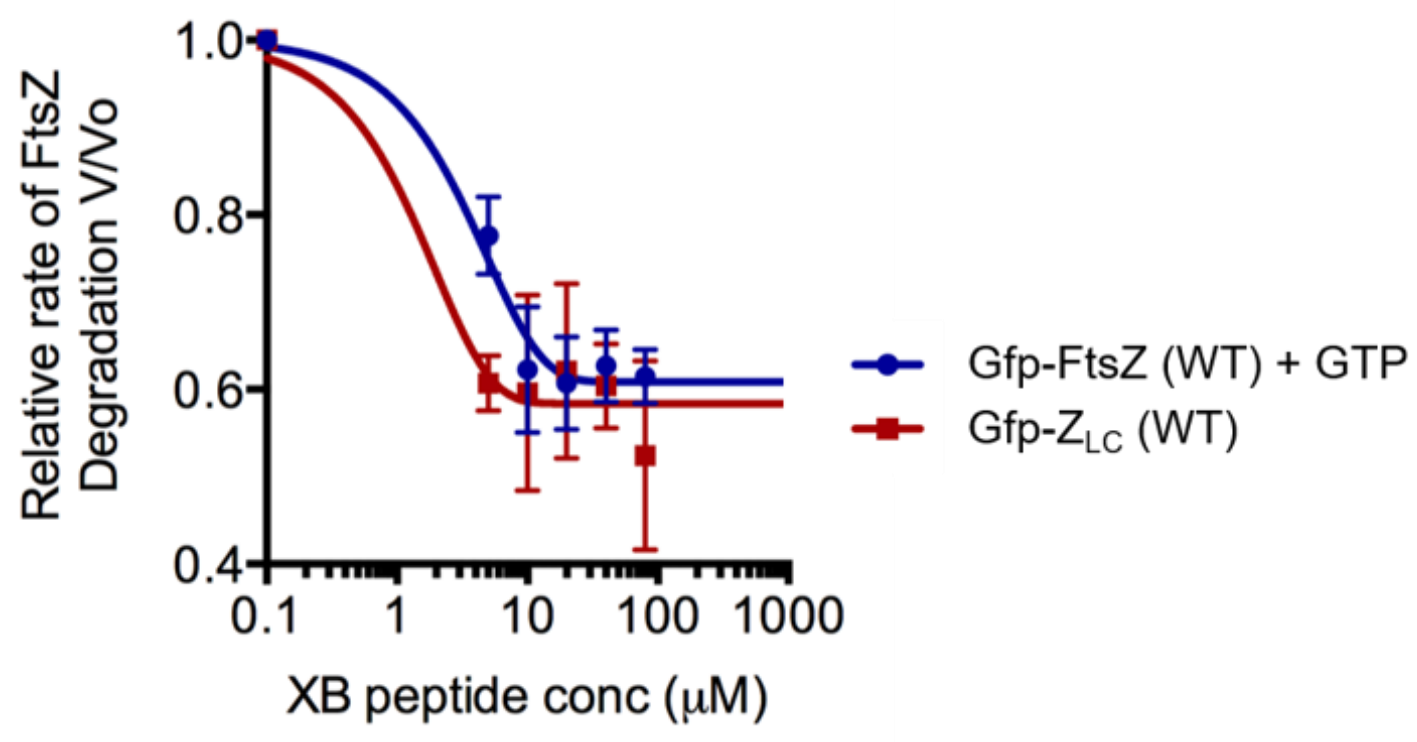




\section{Figure 3. Relative degron position and distance affects FtsZ degradation by ClpXP.}

(A) Schematic for Gfp-FtsZ substrate: fluorescent protein Gfp is fused to the full-length FtsZ protein, including the polymerization domain in purple (residues 1-316); the linker degron is indicated in yellow and the extreme C-terminal degron in cyan. The degrons were swapped (Gfp-FtsZ $Z_{\text {SWAP1 }}$ ), and the space between the swapped degrons was subsequently removed (Gfp-FtsZ $\left.\mathrm{SWAP}_{2}\right)$. (B) Either Gfp-FtsZ $(6 \mu \mathrm{M})$ (white triangles) or Gfp-FtsZ $Z_{\text {SWAP1 }}(6 \mu \mathrm{M})$ (red triangles) was precincubated with GTP $(2 \mathrm{mM})$, and degradation by ClpXP $(1 \mu \mathrm{M})$ with ATP $(5 \mathrm{mM})$ and a regenerating system was monitored with time as described. Reaction buffer was monitored over time for reference (grey triangles). Curves shown are representative of at least three replicates. (C) Either Gfp-FtsZ $(6 \mu \mathrm{M})$ (white triangles) or Gfp-FtsZswAP1 $(6 \mu \mathrm{M})$ (red triangles) were incubated with ClpXP $(1 \mu \mathrm{M})$, ATP $(5 \mathrm{mM})$ and a regenerating system, and degradation was monitored with time as described. Reaction buffer was monitored over time for reference (grey triangles). Curves shown are representative of at least three replicates. (D) Either Gfp-FtsZswAP1 $(6 \mu \mathrm{M})$ (black triangles) or Gfp-FtsZswAP2 $(6 \mu \mathrm{M})$ (red triangles) was precincubated with GTP $(2 \mathrm{mM})$, and degradation by ClpXP $(1 \mu \mathrm{M})$ with ATP $(5$ $\mathrm{mM}$ ) and a regenerating system was monitored with time as described. Reaction buffer was monitored over time for reference (grey triangles). Curves shown are representative of at least three replicates. (E) Either Gfp-FtsZ $\mathrm{SwAP1}_{\mathrm{S}}(6 \mu \mathrm{M})$ (black triangles) or GfpFtsZ $_{\text {SWAP2 }}(6 \mu \mathrm{M})$ (red triangles) were incubated with ClpXP $(1 \mu \mathrm{M})$, ATP $(5 \mathrm{mM})$ and a regenerating system, and degradation was monitored with time as described. Reaction buffer was monitored over time for reference (grey triangles). Curves shown are representative of at least three replicates. 
A

$$
\begin{aligned}
& \text { Gfp-FtsZ } \\
& \text { Gfp-Fts } \\
& \text { SwA-Fts } \\
& \text { GWAP2 }
\end{aligned}
$$

B
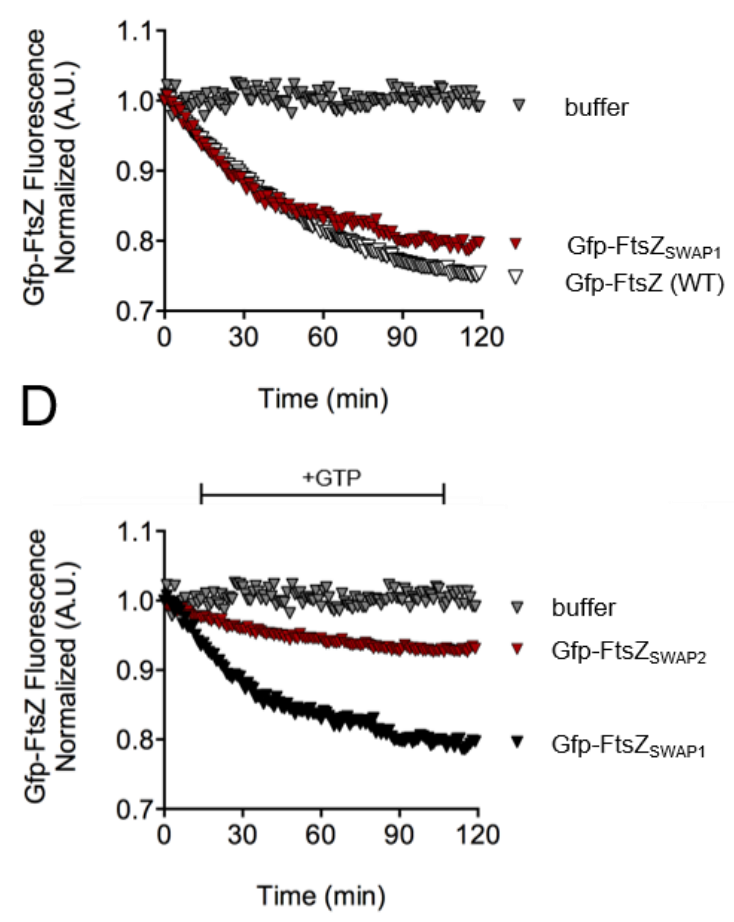

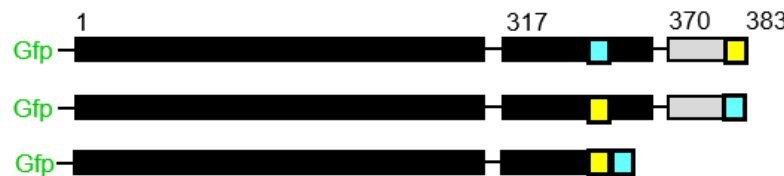

C

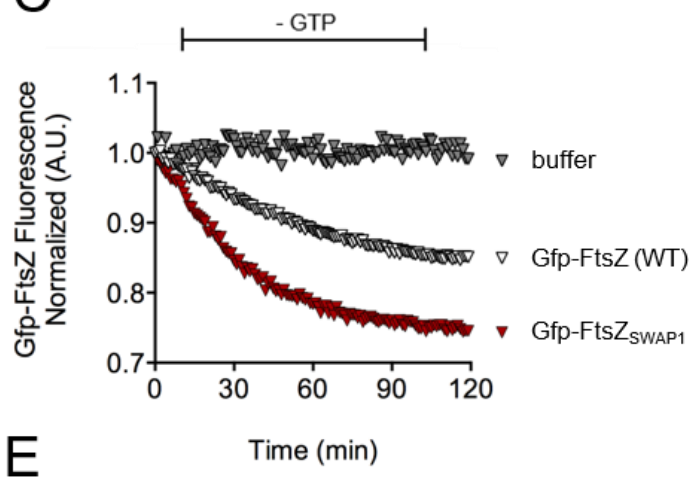

E

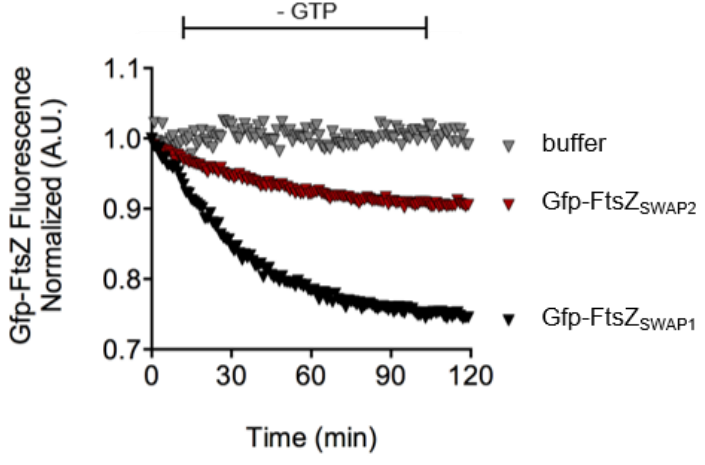


Figure 4. Multimerization is important for recruitment of the FtsZ C-terminus in vivo. (A) DIC and fluorescence microscopy images of wild type MG1655-derived cells in log phase expressing Gfp- $Z_{\mathrm{LC}}$ induced with increasing concentrations of arabinose as described. (B) DIC and fluorescence microscopy images of wild type MG1655-derived cells in log phase expressing Gfp-FtsZ induced with $0.001 \%$ arabinose as described. 
A

B

MG1655 + pGfp- $Z_{L C}$

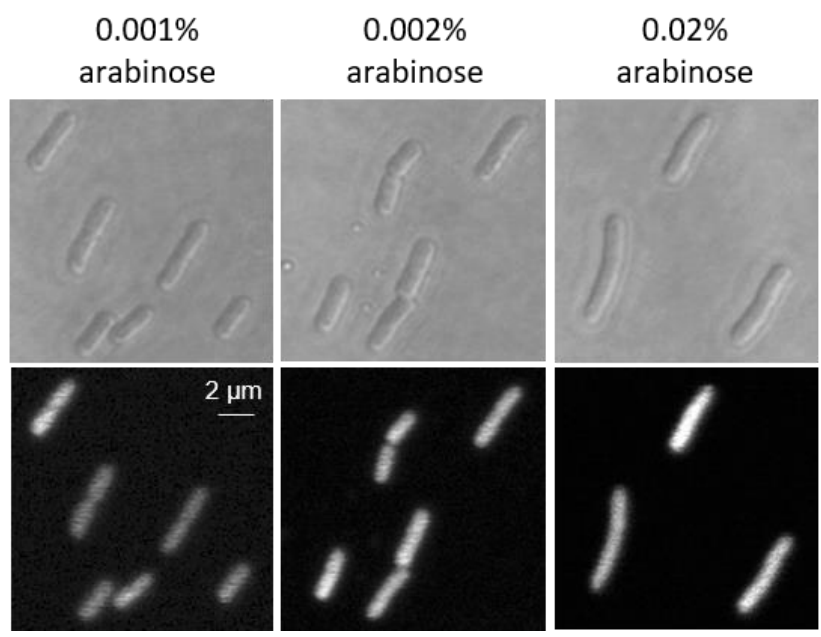

MG1655 + pGfp-FtsZ

$0.001 \%$

arabinose

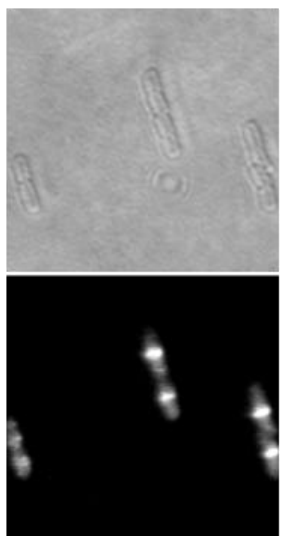


Figure 5. Model for recognition of FtsZ population by ClpXP. For all panels, a crosssection of the ClpX hexamer is shown in blue, docked to a cross-section of the ClpP tetradecamer in green. (A) The Gfp- $Z_{\mathrm{LC}}$ protein, which comprises a barrel-shaped Gfp protein (dark green) fused to the $67 \mathrm{C}$-terminal amino acids of FtsZ, docks the N-domain of ClpX (blue oval) via the C-terminal degron (yellow circle), but not the linker degron (cyan circle). (B) Unassembled FtsZ, which exists as a mixture of dimers and monomers, docks the N-domain of ClpX (blue oval) via the C-terminal degron (yellow circle), but not the linker degron (cyan circle) of FtsZ. The N-domain of FtsZ (purple box) does not bind the ClpX N-domain (blue oval). (C) ClpX recognizes the $\mathrm{C}$-terminal degron (yellow circle) via the $\mathrm{N}$-domain of $\mathrm{ClpX}$ (blue oval), but the linker degron (cyan circle) binds independently of the ClpX N-domain (blue oval) (likely directly in the ClpX axial pore). 
A

B

ClpX

$\mathrm{N}$-domain

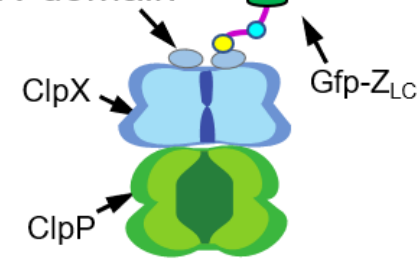

Synthetic monomer

FtsZ

FtsZ

C

FtsZ polymer

dimer

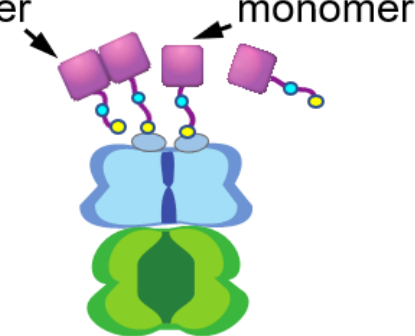

Unassembled FtsZ Polymerized FtsZ 


\section{Manuscript IV}

Publication status: published in PLoS One, 2017

Title: Proteolysis-Dependent Remodeling of the Tubulin Homolog FtsZ at the Division Septum in Escherichia coli

Authors: Marissa G. Viola, Christopher J. LaBreck, Joseph Conti, Jodi L. Camberg*

\section{Author Affiliations:}

Department of Cell and Molecular Biology, The University of Rhode Island, Kingston, Rhode Island, United States of America

*Corresponding author: Jodi L. Camberg, 120 Flagg Road, Kingston, RI, 02881; Tel: (401)874-4961; Email: cambergj@mail.uri.edu 


\begin{abstract}
During bacterial cell division a dynamic protein structure called the Z-ring assembles at the septum. The major protein in the Z-ring in Escherichia coli is FtsZ, a tubulin homolog that polymerizes with GTP. FtsZ is degraded by the two-component ATP-dependent protease ClpXP. Two regions of FtsZ, located outside of the polymerization domain in the unstructured linker and at the $\mathrm{C}$-terminus, are important for specific recognition and degradation by ClpXP. We engineered a synthetic substrate containing green fluorescent protein (Gfp) fused to an extended FtsZ C-terminal tail (residues 317-383), including the unstructured linker and the C-terminal conserved region, but not the polymerization domain, and showed that it is sufficient to target a nonnative substrate for degradation in vitro. To determine if FtsZ degradation regulates Zring assembly during division, we expressed a full length Gfp-FtsZ fusion protein in wild type and $c l p$ deficient strains and monitored fluorescent Z-rings. In cells deleted for $c l p X$ or $c l p P$, or cells expressing protease-defective mutant protein $\mathrm{ClpP}(\mathrm{S} 97 \mathrm{~A})$, Z-rings appear normal; however, after photobleaching a region of the Z-ring, fluorescence recovers $\sim 70 \%$ more slowly in cells without functional ClpXP than in wild type cells. Gfp-FtsZ(R379E), which is defective for degradation by ClpXP, also assembles into Zrings that recover fluorescence $\sim 2$-fold more slowly than Z-rings containing Gfp-FtsZ. In vitro, ClpXP cooperatively degrades and disassembles FtsZ polymers. These results demonstrate that ClpXP is a regulator of Z-ring dynamics and that the regulation is proteolysis-dependent. Our results further show that FtsZ-interacting proteins in E. coli fine-tune Z-ring dynamics.
\end{abstract}




\section{Introduction}

Cell division in bacteria is a conserved and highly coordinated dynamic process involving many cellular proteins that function together to divide a single cell into two daughter cells [1]. During cell division the Z-ring assembles at midcell, the site of septation. The Z-ring contains the essential cell division protein FtsZ and many other division proteins, which are recruited to the septum. FtsZ is a GTPase that is structurally homologous to eukaryotic tubulin and forms large, dynamic polymers [2]. Each FtsZ monomer contains a compact, globular N-terminal polymerization domain, a flexible unstructured linker region, and a conserved region near the C-terminus that is important for protein interactions [르, $\underline{3}]$. Several proteins in E. coli bind to FtsZ and have been shown to modulate the polymerization state of FtsZ in vitro, including MinC, SImA and Z-ring associated proteins (ZAPs) [4]. Many of these protein-protein interactions occur near the FtsZ C-terminus.

High-resolution microscopy of division septa in several organisms, including $E$. coli, Bacillus subtilis, Staphylococcus aureus, Caulobacter crescentus and Streptococcus pneumoniae, showed that the Z-ring contains a network of overlapping FtsZ polymers staggered around the inner face of the cytoplasmic membrane [5-10]. By fluorescence microscopy, the Z-ring is also frequently observed as a loose helical structure rather than a closed ring. The Z-ring is highly dynamic and rapidly exchanges FtsZ subunits with a

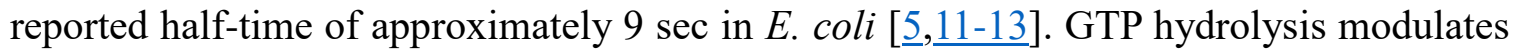
the polymerization state of FtsZ and is thought to be the major factor that promotes dynamic exchange in the Z-ring. An E. coli strain containing the substitution mutation 
G105S in FtsZ impairs GTP hydrolysis in vitro and confers a temperature-sensitive growth phenotype [14-16]. Cells containing chromosomal $f t s Z(G 105 S)$, also referred to as ftsZ84, have Z-rings that exhibit 3-fold slower dynamics, suggesting that Z-ring dynamics are coupled to GTP hydrolysis [12]. It has also been reported that cell division proteins, specifically those that interact directly with FtsZ, may modulate Z-ring dynamics in $E$. coli. FtsZ-interacting proteins ZapA and ZapB were shown to stabilize the network of overlapping FtsZ polymers in Z-rings in vivo and promote polymer bundling in vitro [1719].

Counteracting the functions of proteins that stabilize or bundle FtsZ polymers, several proteins antagonize FtsZ polymers and promote their disassembly. In E. coli, MinC, SlmA, ClpXP and most recently ZapE have been reported to destabilize FtsZ polymers in vitro by promoting disassembly, preventing reassembly or by shifting the equilibrium of FtsZ polymers towards disassembly $[4, \underline{20-26]}$. Deletion of the $\min C D E$ operon from E. coli leads to the assembly of Z-rings with $\sim 2$-fold slower dynamics [12]. The Min system in E. coli, which includes MinC, MinD and MinE, inhibits Z-ring assembly at non-septal locations by establishing an oscillating polar gradient of MinC, an FtsZ polymerization inhibitor [27]. The contributions of SlmA, which prevents polymerization of FtsZ over the nucleoid, and ZapE, which may destabilize FtsZ polymers during late constriction, to Z-ring dynamics are unknown.

Proteolysis is an important regulatory mechanism for the cell division pathway in bacteria. ClpXP is an ATP-dependent protease that contains the ATP-dependent chaperone unfoldase, $\mathrm{ClpX}$, bound to a compartmentalized protease, ClpP [28]. In $C$. crescentus, ClpXP controls initiation of DNA replication through degradation of CtrA and 
is regulated through phosphoregulated adaptor complexes [29]. In addition, ClpXP degrades FtsZ in non-replicative swarmer cells [30].

In E. coli $\mathrm{ClpXP}$ is known to degrade many diverse substrates $[\underline{31}, \underline{32}]$. ClpXP specifically recognizes, unfolds, and degrades FtsZ in vivo and in vitro, and overexpression of ClpXP leads to cell filamentation and increased degradation of FtsZ [24]. ClpXP degrades approximately $15 \%$ of total FtsZ per cell cycle in E. coli [24]. Furthermore, the ClpX chaperones from E. coli, B. subtilis, and Mycobacterium tuberculosis have also been shown to antagonize FtsZ polymerization in the absence of ClpP [33-36].

$\mathrm{ClpX}$ is a member of the AAA+ superfamily of ATPases and forms a hexameric ring with a central substrate translocation channel. Several eukaryotic AAA+ ATPases, such as spastin and katanin, have been suggested to sever microtubules and alter tubulin dynamics in vivo [37-41]. Spastin and katanin are members of a meiotic clade of AAA+ ATPases, which, along with Vps4, may disassemble polymers [40]. ClpX from E. coli promotes disassembly and degradation of FtsZ polymers along with its cognate protease ClpP [24]. Two regions of FtsZ are important for recognition and degradation by ClpXP. One region is present in the unstructured linker (residues 352-358) of FtsZ and the other is present near the C-terminus (residues 379-383) [25]. Although FtsZ monomers and polymers are degraded by ClpXP, FtsZ polymers, containing either GTP or the GTP analog GMPCPP, are degraded more efficiently $[\underline{24}, \underline{25}, \underline{42}]$.

Here, we demonstrate that the C-terminal extended tail of FtsZ, which includes the unstructured linker and conserved C-terminal region, but not the polymerization domain, is sufficient to target a non-native substrate for degradation by ClpXP in vitro. To 
uncover the functional role of FtsZ proteolysis by ClpXP during division, we expressed N-terminal Gfp-FtsZ fusion proteins in cells and monitored fluorescence recovery after bleaching Z-rings in vivo. We show that cells unable to make functional ClpXP, or cells that express an FtsZ mutant protein defective for degradation, have Z-rings that recover fluorescence more slowly than Z-rings in wild type cells. We further show that in vitro, degradation of FtsZ polymers occurs cooperatively, indicating that polymerization of FtsZ promotes recognition by ClpX. Together, these results demonstrate that the role for ClpXP during the division cycle of E. coli is to engage the FtsZ C-terminal region, degrade FtsZ and regulate FtsZ polymer dynamics.

\section{Results}

Targeting and degradation of a synthetic fluorescent substrate by addition of the FtsZ extended C-terminus

Recognition and degradation of FtsZ by ClpXP was previously shown by our group to utilize two regions of FtsZ, residues 352-358 in the unstructured linker, and Cterminal residues 379-383 [25]. To determine if an extended C-terminal tail of FtsZ, including the unstructured linker (residues 317-369), the conserved region adjacent to the FtsZ C-terminus (residues 370-379) and the C-terminal variable region (residues 380383 ) is sufficient to target a synthetic substrate for degradation, we constructed and purified a fluorescent fusion protein containing Gfp and the extended FtsZ C-terminal tail (Gfp-Z $\left.\mathrm{Z}_{\mathrm{C} 67}\right)$ (Fig 1A). This chimera does not contain the FtsZ polymerization domain. We measured degradation of Gfp- $Z_{\mathrm{C} 67}$ in reactions containing $\mathrm{ClpXP}$ and ATP by monitoring loss of fluorescence during incubation with ClpXP, indicating that ClpXP unfolds and 
degrades the Gfp moiety (Fig 1B). After 60 min, approximately $25 \%$ of the initial fluorescence was lost under the conditions tested, and then the degradation reaction plateaued (Fig 1B). Gfp without $\mathrm{Z}_{\mathrm{C} 67}$ is stable and not degraded during incubation with ClpXP since it does not contain a ClpX-recognition region (S1A Fig). We also observed that a Gfp-fusion protein containing full-length FtsZ is degraded by ClpXP (Fig 1A and 1C). The loss of fluorescence is attributable to degradation since incubation of Gfp- $Z_{\mathrm{C} 67}$ or Gfp-FtsZ with ClpX alone, without ClpP, has no effect on the fluorescence of either substrate (S1B Fig). The addition of GTP, which is known to induce FtsZ polymerization, to the degradation reaction containing Gfp-FtsZ increases the rate of degradation from 0.008 units $\mathrm{min}^{-1}$ to 0.016 units $\mathrm{min}^{-1}$ (Fig 1C). This is in agreement with previous reports showing a 2-3-fold increase in degradation efficiency for native FtsZ in the presence of GTP and suggests that Gfp-FtsZ is capable of polymerization with GTP $[\underline{24}, \underline{25}]$. To confirm this, we performed sedimentation assays using mixtures of FtsZ and Gfp-FtsZ. We observed Gfp-FtsZ sedimentation in the presence of GTP alone and when increasing amounts of FtsZ are included in the reaction, indicating that Gfp-FtsZ polymerizes with GTP (Fig 1D).

\section{Z-ring localization of Gfp-FtsZ in dividing cells and slow dynamics in $c l p$ deletion strains}

Next, we wanted to determine if Z-ring assembly and dynamics are perturbed in cells lacking ClpXP. To localize fluorescent Z-rings in live, dividing cells, we expressed Gfp-FtsZ from a plasmid in a wild type E. coli MG1655 strain (JC0390), and in strains deleted for $\operatorname{clpX}$ (JC0394) or $\operatorname{clpP}$ (MV0210) (Table 1). Importantly, we used the Nterminal Gfp-FtsZ fusion protein, which is competent for polymerization (Fig 1D) and 
leaves the C-terminal ClpX interaction site of FtsZ accessible. All strains also constitutively express an arabinose transporter $\left(\mathrm{P}_{\mathrm{CP} 18-a r a E)}\right)$ to reduce cell-to-cell variability in the presence of inducer (arabinose) [녀․

Expression of Gfp-FtsZ in the wild type strain (JC0390) does not interfere with Zring assembly or division under the expression conditions tested and constitutes $\sim 50 \%$ of the total FtsZ in the cell (Fig 1E and 1F). As expected, fluorescent Z-rings were present at the center of the long axis of dividing cells and the rings contained approximately 20$30 \%$ of the total cellular fluorescence (Fig $1 \mathrm{G}$ and $1 \mathrm{H}$ ). To probe the dynamic exchange of fluorescent subunits in the Z-ring of individual dividing cells, we selected a small region of the fluorescent Z-ring in each cell and performed a photobleaching and recovery assay (Fig 2A). After bleaching the fluorescence from the region, recovery in the selected area was monitored at 3,6 or $8 \mathrm{sec}$ intervals over the next $72 \mathrm{sec}$, as GfpFtsZ from within the Z-ring is exchanged with subunits from the cytoplasm (Fig 2A, 2B and $2 \mathrm{C}$ ). During the recovery period, we measured the fluorescence at each interval for each region, calculated the recovery half-time, and then determined the average recovery half-time for all replicates. The fluorescence recovery half-time of Z-rings in wild type, dividing cells expressing Gfp-FtsZ is $6.2 \pm 0.5 \mathrm{sec}(\mathrm{n}=18,8 \mathrm{sec}$ interval) (Fig 2C) (S1 Table), which is faster but within error of half-times reported previously $(9.0 \pm 3$ and 8.3 $\pm 3 \mathrm{sec}$ for wild type Z-rings in E. coli and B. subtilis respectively) [12]. Recoveries measured at shorter capture intervals $(6 \mathrm{sec}$ and $3 \mathrm{sec}$ ) generated average half-time values similar to the value measured using an $8 \mathrm{sec}$ interval $(6.8 \pm 0.5 \mathrm{sec}$ and $7.5 \pm 0.5 \mathrm{sec}$, respectively) (Fig 2C).

Next, we expressed Gfp-FtsZ in cells deleted for $\operatorname{clp} X$ or $\operatorname{clp} P$, and observed 
fluorescent Z-rings at midcell, and cells appeared similar to wild type cells expressing Gfp-FtsZ (Fig 1G, 3A and 3B) (S2A Fig). We also constructed a strain in which the chromosomal $c l p P$ gene was replaced with a gene at the native locus encoding proteasedefective $\operatorname{clp} P(S 97 A)$ and visualized Z-rings in ClpP-defective cells by expression of Gfp-FtsZ (Fig 3C and 3D) (S2A Fig) [499, $\underline{50}$. We observed no apparent cell division or morphological defects in the strain expressing $\mathrm{ClpP}(\mathrm{S} 97 \mathrm{~A})$ and Z-rings appeared similar to Z-rings in wild type and $c l p P$ deletion strains (Fig 1G) (S2A Fig). Next, we selected and bleached regions of fluorescent Z-rings in clp deficient cells and monitored fluorescence recovery. Surprisingly, in all $c l p$ deficient strains, we observed an average recovery half-time significantly longer by $70 \%$ than the half-time observed in the parental strain (average recovery half-time of the Z-ring is $10.6 \pm 1.3 \mathrm{sec}$ in the $\Delta c l p X$ strain, 10.6 $\pm 0.7 \mathrm{sec}$ in the $\triangle c l p P$ strain, and $10.4 \pm 0.4 \mathrm{sec}$ in the $c l p P(S 97 A)$ strain) (Fig 3E) (S2B, S2C and S2D Figs) (S1 Table). We also observed that the distributions of values are larger in $c l p$ deficient strains, suggesting that there is more heterogeneity among the Zrings, which could indicate that there is less control over regulating Z-ring architecture in a given population of cells. Finally, we restored wild type copies of $\operatorname{clp} X$ and $\operatorname{clp} P$ to $c l p$ deletion strains at their native loci by recombination, expressed Gfp-FtsZ from a plasmid in the restored strain and performed bleaching and recovery assays of fluorescent Z-rings to determine if we could rescue the slow average recovery half-time (S2A, S2E, S2F, S2G and S2H Figs) (Table 1). We observed that strains restored with $c l p X$ (MV03722) or clpP (MV03712) at the native locus have average half-time recoveries of $8.2 \pm 1.4 \mathrm{sec}$ and $8.1 \pm 0.6 \mathrm{sec}$, respectively. Although the values are not significantly different from the value observed for the wild type parental strain (JC0390), it is interesting to note that 
the strain restored with $\operatorname{clp} X$ has a slightly lower level of ClpX expression than the wild type strain, which could explain the modest increase in recovery half-time compared to the wild type strain (S1 Table).

Deletion of $c l p X$ or $c l p P$ prevents degradation of FtsZ in vivo, leading to slower FtsZ protein turnover as measured in antibiotic chase assays [24]. To test if slow Z-ring fluorescence recovery could be attributable to higher levels of Gfp-FtsZ, we directly tested if a higher expression level of Gfp-FtsZ also leads to slower recovery, but did not observe a significant difference in recovery half-time at a higher arabinose concentration $(7.5 \pm 0.8 \mathrm{sec})($ Fig S3A and S3B). This is in agreement with a previous report showing that an increase in FtsZ expression does not alter the architecture of the Z-ring [ $\underline{5}$. These results show that Z-rings in cells lacking functional ClpXP have slow recovery after bleaching and are less dynamic in early division.

\section{A residue in the Fts $Z$ C-terminal conserved region is important for fast exchange in the Z-ring and recognition by ClpXP}

The FtsZ mutant protein FtsZ(R379E) has a substitution at the end of the Cterminal conserved region and is defective for degradation by ClpXP in vitro [25]. To determine if Arg 379 is critical for targeting ClpX, we introduced the R379E substitution into Gfp- $Z_{\mathrm{C} 67}$, and measured the degradation of purified $\mathrm{Gfp}-Z_{\mathrm{C} 67}(\mathrm{R} 379 \mathrm{E})$ by $\mathrm{ClpXP}$ in vitro by monitoring the loss of fluorescence with time. We observed that Gfp$\mathrm{Z}_{\mathrm{C} 67}(\mathrm{R} 379 \mathrm{E})$ is degraded $\sim 50 \%$ more slowly than $\mathrm{Gfp}-\mathrm{Z}_{\mathrm{C} 67}$ indicating that $\mathrm{Arg} 379$ is important for recognition and degradation by ClpXP (Fig 4A).

To investigate if FtsZ(R379E) is capable of assembly into functional Z-rings, we expressed Gfp-FtsZ(R379E) in the wild type parental strain (JC0390). Expression of Gfp- 
FtsZ(R379E) causes cells to be 2.7-fold longer than cells expressing Gfp-FtsZ $(9.6 \pm 0.6$ $\mu \mathrm{m}$ and $3.5 \pm 0.1 \mu \mathrm{m}$, respectively), and the cells have multiple Z-rings and additional fluorescent foci at sporadic locations (Fig 4B) (S4A Fig) (S1 Table). To investigate if the R379E substitution near the FtsZ C-terminus impairs the dynamic exchange of subunits in the Z-ring, we performed bleaching and recovery assays in wild type cells expressing Gfp-FtsZ(R379E) from a plasmid (S4B Fig). Expression of Gfp-FtsZ(R379E) in the wild type strain results in the assembly of a Z-ring that recovers fluorescence with a delayed average half-time of $12.1 \pm 1.6 \mathrm{sec}$, which is 2-fold slower than Gfp-FtsZ (Fig 4C). Moreover, the individual half-time values are broadly distributed, similar to what we observed in $c l p$ deletion strains (Fig 4C and 3E) (S1 Table). These results suggest that Arg 379 in FtsZ is important for promoting fast exchange of FtsZ subunits in the Z-ring and is consistent with the role of ClpXP. Moreover, slow fluorescence recovery is not due to defective GTP hydrolysis or polymerization, since FtsZ(R379E) hydrolyzes GTP and assembles in vitro (S4E Fig) [25]. Furthermore, the expression levels of Gfp-FtsZ and Gfp-FtsZ(R379E) in vivo were similar for both strains (S4F Fig).

Mutation of G105S in FtsZ impairs GTP hydrolysis in vitro and confers a temperature-sensitive, filamentous phenotype in vivo; however, FtsZ(G105S) is capable of polymerization in vitro $[\underline{14-16}, \underline{51-53}]$. Cells expressing fluorescent Gfp-FtsZ(G105S) have previously been shown to exhibit slow fluorescence recovery after bleaching, even when grown at the permissive temperature, suggesting that the rate of GTP hydrolysis is coupled to subunit exchange in the Z-ring $[\underline{12}, \underline{13}]$. To confirm that impaired GTP hydrolysis also slows subunit exchange in vivo with the N-terminal Gfp-FtsZ fusion protein used in this study, we expressed Gfp-FtsZ(G105S) in the parental strain 
containing wild type $f t s Z$ on the chromosome and measured fluorescence recovery after bleaching in the Z-ring (Fig 4B and 4C) (S4C Fig). We observed that Z-rings containing Gfp-FtsZ(G105S) recover fluorescence $\sim 2$-fold more slowly than cells expressing GfpFtsZ (12.6 $\pm 1.5 \mathrm{sec}$ and $6.2 \pm 0.5 \mathrm{sec}$, respectively) (Fig 4C) (S1 Table). Since mutation of either site (G105 or R379) is important, we tested if impairing both would further slow dynamic exchange in the Z-ring. We constructed Gfp-FtsZ(G105S, R379E) and visualized Z-rings in wild type cells. Gfp-FtsZ(G105S, R379E) localizes to Z-rings, but the cells are filamentous. We monitored fluorescence recovery in Z-rings but detected no further slowdown than observed for either mutation individually (Fig 4C) (S4D Fig) (S1 Table). We again observed a wide distribution of recovery times within the data set, compared to the parental strain expressing Gfp-FtsZ, and this heterogeneity may suggest a defect in regulating Z-ring assembly or maintenance. The expression levels of Gfp-FtsZ mutant proteins were similar to the level observed in cells expressing wild type Gfp-FtsZ (S4F Fig). As expected, we also observed that incorporation of the G105S mutation slows the rate of GTP hydrolysis of both FtsZ and FtsZ(R379E) using purified proteins in vitro (S4E Fig).

Next, we engineered alanine substitutions in the unstructured linker region of FtsZ spanning a second $\mathrm{ClpX}$ interaction site (residues 352-358) to construct FtsZ(3527A). We purified FtsZ(3527A) and confirmed that it hydrolyzes GTP and is partially defective for degradation by ClpXP in vitro (S4E and S5A Figs). We also constructed Gfp-FtsZ(3527A) and expressed it in dividing cells. We observed no obvious morphological defects and Zrings appeared similar to cells expressing Gfp-FtsZ (Fig 4B). In bleaching and recovery assays of Z-rings containing Gfp-FtsZ(3527A), we observed a slightly wider distribution 
of recovery values than in rings containing Gfp-FtsZ, but the difference in average recovery half-time is not significant compared to Gfp-FtsZ (Fig 4C) (S5B Fig) (S1 Table).

\section{ClpX does not affect the dynamics of the Z-ring reporter ZipA-Gfp}

To determine if the effect that ClpXP has on fluorescence recovery time in the Zring is restricted to FtsZ or is observable using proteins that localize to a Z-ring after FtsZ localizes to the ring, we used cells expressing ZipA-Gfp from the chromosome and measured Z-ring dynamics in strains with and without $\operatorname{clp} X$ (Fig 5A) (Table 1). Midcell rings containing ZipA-Gfp are a marker for Z-ring formation and have a fast average recovery half-time, similar to the rate observed for Z-rings containing Gfp-FtsZ [13]. ZipA is not a putative substrate for ClpXP degradation and overproduction of ZipA has even been shown to protect FtsZ from degradation by ClpXP [54]. Upon expression of ZipA-Gfp, we observed fluorescent rings at midcell and cells appeared normal in length with and without chromosomally-encoded $\operatorname{clp} X$ under the conditions tested (MC181 and MV0226) (Fig 5A). We performed bleaching and fluorescence recovery assays on ZipAGfp rings in dividing cells with and without $\operatorname{clp} X$ and observed nearly identical average recovery half-times for ZipA-Gfp rings in both strains, $5.8 \pm 0.6 \mathrm{sec}$ in cells containing $c l p X$ and $5.8 \pm 0.5 \mathrm{sec}$ in cells deleted for $\operatorname{clp} X$ (Fig 5B) (S1 Table). Both ZipA-Gfp average recovery half-times are within error of Gfp-FtsZ in the wild type parental strain (JC0390) and unaffected by the presence or absence of ClpX (S1 Table).

\section{Cooperative degradation and disassembly of FtsZ polymers by ClpXP}


FtsZ polymers and unassembled FtsZ, which is a mixture of monomers and dimers, are degraded by ClpXP. In vitro, degradation reduces the overall abundance of polymers, and this is thought to occur as a result of degrading both populations, shifting the dynamic equilibrium of polymerized FtsZ in the direction of disassembly [24]. To determine if ClpXP reduces the size of existing FtsZ polymers, which would favor a directed disassembly model, we incubated ClpXP with FtsZ polymers, crosslinked the reaction products, and then analyzed their relative sizes by fractionation on a sucrose gradient. Using labeled fluorescent FtsZ (FtsZ-AF488) we observed that without GTP, FtsZ exists as a mixture of monomers $(40.4 \mathrm{kDa})$ and dimers (80.8 kDa) (Fig 6A); however, when FtsZ was incubated with GTP and then crosslinked, we observed a large peak present at the high sucrose concentration $(>440 \mathrm{kDa})$, corresponding to fractions 1 through 3 , which together contained $25.1 \%$ of the total fluorescence in the initial reaction. We also detected fluorescence in the region between $158 \mathrm{kDa}$ and $440 \mathrm{kDa}$, corresponding to fractions 4 through 6 , which is consistent with short polymers and represents $14.5 \%$ of the total fluorescence (Fig. 6A). In contrast, in the absence of GTP, minimal fluorescence was detected in regions corresponding to polymers (fraction 1 through 3) and short polymer fragments (fractions 4 through 6) amounting to $5.2 \%$ and $5.3 \%$ of the total fluorescence, respectively (Fig. 6A). These results show that although FtsZ polymers formed in the presence of GTP are dynamic, incubation with dithiobis(succinimidyl propionate) (DSP) crosslinks a significant population of polymerized FtsZ, with the majority of polymers being very large. Next, we used this assay to test if incubation of FtsZ with ClpXP reduces the amount of large polymers detected. FtsZ polymers were assembled with GTP and then incubated with ClpXP prior 
to crosslinking. After incubation with ClpXP, we failed to detect a significant population of large polymers present in fractions 1 through 3 above the level of background (6.1\% of total fluorescence) and even observed that there was less fluorescence in fractions 4 through 6 , which corresponds to the region likely to contain short polymers, $10.1 \%$ of the total fluorescence with ClpXP, compared to $14.5 \%$ of the total fluorescence without ClpXP in fractions 4 through 6 (Fig 6A). Small molecular weight fluorescence was also detected in the reaction containing ClpXP in the low percent sucrose fraction due to the accumulation of fluorescent peptides, which are the products of degradation. However, the total fraction of fluorescent peptides was small, since FtsZ was in large excess over ClpXP. These results suggest that ClpXP disassembles large FtsZ polymers through degradation.

Finally, we used this assay to determine if ClpX alone, in the absence of ClpP, could also lead to fewer large FtsZ polymers, since ClpX has been reported to interact directly with FtsZ in vitro [36]. We observed a small reduction in the amount of fluorescence detected in polymer fractions 1 through 3 amounting to $15.4 \%$ of the total fluorescence with $\mathrm{ClpX}$, compared to $25.1 \%$ of the total fluorescence without ClpX (but with GTP) in fractions 1 through 3 (Fig. 6A). These results suggest that ClpX can modestly promote disassembly of large FtsZ polymers, but destabilization is more efficient in the presence of ClpP.

Interactions between FtsZ and several FtsZ-interacting proteins, including ZipA and SlmA, are mediated by multivalent interactions with FtsZ subunits within a polymer [42]. In addition, the rate of FtsZ degradation by ClpXP is enhanced when FtsZ is 
polymerized with GTP (Fig 1C) or the analog GMPCPP, as previously shown $[24,25]$. To determine if FtsZ polymers are recognized and degraded in a cooperative manner, we compared the rates of FtsZ degradation by ClpXP at concentrations above the critical concentration for FtsZ polymerization $(1 \mu \mathrm{M})$ [2]. We observed that as the FtsZ concentration increased, the rate of degradation also increased in a positively cooperative manner $\left(\mathrm{n}_{\mathrm{h}}=1.7\right)$ when GTP was present (Fig 6B). In contrast, no cooperativity was observed when GTP was omitted from the degradation reactions $\left(\mathrm{n}_{\mathrm{h}}=1.0\right)$ and the overall rate of degradation was lower than in the presence of GTP. These results suggest that degradation of FtsZ polymers by ClpXP occurs by a cooperative recognition mechanism.

\section{Disassembly of FtsZ polymers is a mechanism to modulate Z-ring dynamics}

There are relatively few reports of factors that are capable of modifying Z-ring dynamics in E. coli. One previous report showed that deletion of the minCDE operon from E. coli slows Z-ring dynamics approximately 2 -fold, similar to the effects observed here in $c l p$ deficient strains (Fig 3E) [12]. Since both MinC and ClpXP can destabilize FtsZ polymers in vitro, we investigated if destabilization of FtsZ polymers may be a mechanism to modulate Z-ring dynamics. Therefore, we expressed Gfp-FtsZ in strains deleted for $\min C$ (JC0395), $\operatorname{sim} A$ (MV0198), or zapE (MV0277), all of which are reported to promote FtsZ polymer disassembly, visualized fluorescent Z-rings and performed bleaching and recovery assays (Fig 7) (S6A and S6B Figs). In a $\min C$ deletion strain expressing Gfp-FtsZ, cells are mildly filamentous and contain multiple Z-rings (Fig 7A), which agrees with previous reports that deletion of $\min C$ leads to the formation of anucleate minicells as well as elongated cells with an increased number of Z-rings per 


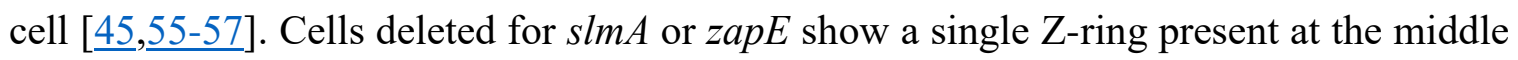
of the long-axis of the cell and are similar in length to the wild type strain (Fig 7B and 7C) (S1 Table). In all strains, approximately $20-30 \%$ of the total cellular fluorescence was present in a Z-ring (data not shown), similar to the wild type strain (Fig 1H). Next, we monitored fluorescence recovery of Z-rings in cells deleted for $\min C, \operatorname{sim} A$, or zapE. As expected, in cells deleted for $\min C$, we observed a $70 \%$ longer average fluorescence recovery half-time than in the parental strain (Fig 7D) (S1 Table). Furthermore, reinsertion of $\min C$ back into the native locus by lambda-Red recombination restored MinC expression and rescued the slow Z-ring recovery of cells expressing Gfp-FtsZ to wild type half-times (S6A, S6C and S6D Figs) (S1 Table). We also observed slow Z-ring fluorescence recovery in the strain deleted for $\operatorname{sim} A$, which showed a fluorescence recovery half-time that is on average $80 \%$ slower than in the wild type strain (Fig 7D) (S1 Table). Z-ring subunit exchange in cells deleted for zapE occurred faster than in the other deletion strains tested, but 50\% slower than the parental strain (Fig 7D) (S1 Table). Taken together, our results suggest that proteins that function to destabilize FtsZ polymers in vitro enhance dynamic exchange in the Z-ring in vivo. Moreover, removal of $\min C$ and $\operatorname{sim} A$ widens the distribution of recovery values among the cells studied, indicating that there is increased heterogeneity throughout the population.

\section{Discussion}

Using direct biochemical degradation assays, we demonstrate that an extended FtsZ C-terminal region (317-383), which includes two sites previously identified as important for ClpX recognition, is sufficient to target the non-native substrate Gfp for degradation (Fig 1B). We also show that full length FtsZ fused to Gfp at the N-terminus 
assembles into polymers with GTP and is also degraded by ClpXP in vitro (Fig 1C and 1D). As expected, degradation of Gfp-FtsZ occurs more rapidly in the presence of GTP, which promotes assembly of FtsZ into polymers. Titrating the FtsZ concentration above the critical concentration for polymer assembly shows a cooperative increase in the rate of degradation by ClpXP, which is consistent with multimerization enhancing recognition through concentration of FtsZ C-terminal binding sites (Fig 6B). In vivo Gfp-FtsZ localizes to the Z-ring, consistent with a previous report using a similarly constructed Nterminal Gfp-FtsZ fusion protein [58]. The polymerization domain is critical for FtsZ incorporation into the $\mathrm{Z}$-ring because $\mathrm{Gfp}-\mathrm{Z}_{\mathrm{C} 67}$ does not localize to a ring (data not shown), even though the protein interaction site near the C-terminus is intact.

The role of ClpXP during cell cycle progression in C. crescentus is wellcharacterized, but in E. coli, the functional role of ClpXP during division was unknown. Here we demonstrate that ClpXP is a regulator of Z-ring dynamics in vivo during division and that the regulation is proteolysis-dependent in vivo. In cells expressing GfpFtsZ, deletion of $\operatorname{clp} X$ or $c l p P$ leads to the formation of Z-rings that are on average $\sim 70 \%$ slower to recover fluorescence after bleaching than Z-rings in the wild type parental strain. We also observed a large distribution of fluorescence recovery half-times, suggesting that although the cells appear normal, there is cell-to-cell variability in the stability of the Z-ring and the cells may be less resistant than wild type cells to further perturbations at the septa. Increased sensitivity may also explain why $c l p$ deletions are synthetic filamentous with a $\min C$ deletion [45]. By replacing the $c l p P$ gene with one that encodes a ClpP proteolytic mutant, ClpP(S97A), we confirmed that ClpXP degradation, not just ATP-dependent remodeling by $\mathrm{ClpX}$, is critical for regulating division. Through 
direct degradation of FtsZ and the additional degradation of the Z-ring stabilizer ZapC, ClpXP likely has potent Z-ring destabilizing activity [두].

The dynamic nature of the Z-ring is widely reported, and other cell division proteins, including FtsA and ZipA, are known to localize to the dynamic ring $[\underline{13}, \underline{60}]$. How dynamics impact progression through the cell cycle is not well understood. Slow GTP hydrolysis by FtsZ is also linked to slow ring dynamics in vivo and was reported for Z-rings containing FtsZ(G105S) [13]. Constriction time could correlate with Z-ring dynamics. In a recent report by the Xiao group, deletion of matP was shown to shorten constriction time. Since MatP stabilizes the Z-ring through interactions with ZapA and ZapB, this suggests that FtsZ-interacting proteins modify the rate of constriction [17]. Interestingly, the dynamic ZipA-Gfp ring, often used as a marker for Z-ring assembly, is not modulated by ClpXP (Fig 5B). A recent study reported that although FtsZ and ZipA are thought to assemble early and form a proto-ring along with FtsA, FtsZ disassembles from the division septum prior to ZipA, suggesting that septal association of FtsZ and ZipA is differentially controlled [2, $\underline{61-63}]$. The important role that FtsZ assembly regulators have in promoting division was recently demonstrated by showing that intragenic suppressor mutations in $f t s Z(G 105 S)$ support division but rely on other cell division proteins, including ZapA [64].

Recognition of FtsZ by ClpX is complex and not yet fully understood. Although two sites in FtsZ are important for ClpXP degradation, the relative contributions of each site to degradation of distinct FtsZ conformations have yet to be determined. One may function as an auto-adaptor or enhancer, while the other may function as the degron, similar to the distinct sites of the ClpX substrate MuA [65]. Mutation of Arg 379 in native 
FtsZ or in Gfp- $Z_{667}$ impairs degradation by ClpXP in vitro (Fig 4A) [25]. Consistent with this, incorporation of Gfp-FtsZ(R379E) into Z-rings in vivo results in Z-rings that recover fluorescence more slowly than cells containing Gfp-FtsZ, suggesting that they are less dynamic due to defective degradation by ClpXP. The extreme C-terminus, also referred to as the $\mathrm{C}$-terminal variable region, mediates bundling in other organisms such as $B$. subtilis, but not in E. coli [66]. We also investigated the second region of FtsZ in the unstructured linker that is involved in ClpX recognition, residues 352 through 358. Although FtsZ(3527A) is degraded more slowly than wild type FtsZ in vitro (S5A Fig), expression of Gfp-FtsZ(3527A) does not significantly perturb Z-ring assembly or fluorescence recovery in the Z-ring (Fig 4B and 4C) (S1 Table). Together, this suggests that the ClpX-interaction site near the FtsZ C-terminus is critical during division. Arg 379 may also be important for recognition by FtsA, which, along with ZipA, recruits FtsZ to the membrane in vivo $[\underline{25}, \underline{58}, \underline{67-69}]$. Arg 379 is well conserved and also present in FtsZ from Thermotoga maritima [TmFtsZ(Arg344)] [70,71]. Accordingly, co-crystals of $T$. maritima FtsA and the C-terminal FtsZ peptide show that TmFtsZ(Arg344) forms a salt bridge with subdomain 2B of FtsA [70].

We also report that deletion of $\min C, \operatorname{sim} A$ and $z a p E$ leads to Z-rings with slower dynamics. In a previous report, deletion of the $\min C D E$ operon was shown to slow the dynamic exchange in the Z-ring to a similar extent observed here by deletion of $\min C$ [12]. Notably, ZapA and ZapB, which stabilize FtsZ polymers in vitro, have been reported to stabilize the Z-ring in vivo $[\underline{11}, \underline{17}]$. It is therefore likely that a global function of FtsZ assembly modulators is to regulate the flux of subunits into and out of the Z-ring. In this way, the network of regulators ensures that appropriate exchange is maintained in 
the Z-ring during division.

\section{Materials and methods}

\section{Bacterial strains, plasmids, and growth conditions}

E. coli strains and plasmids used in this study are described in Table 1. Strains were grown at $30^{\circ} \mathrm{C}$ in Lennox broth supplemented with appropriate antibiotics (kanamycin $50 \mu \mathrm{g} \mathrm{ml}^{-1}$, chloramphenicol $35 \mu \mathrm{g} \mathrm{ml}^{-1}$, and ampicillin $100 \mu \mathrm{g} \mathrm{ml}{ }^{-1}$, where indicated). All MG1655 wild type and deletion strains used in bleaching and recovery assays contain constitutive promoter $\mathrm{P}_{\mathrm{CP} 18}$ in place of the chromosomal araE promoter, introduced by $\mathrm{P} 1$ transduction, to normalize cell-to-cell variation in expression [43]. Single gene kanamycin insertion-deletions were brought into MG1655 by P1 transduction using donor strains from the Keio collection [46]. For construction of histidine tagged

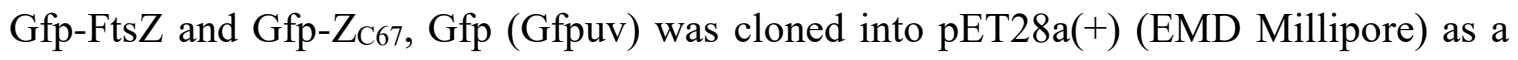
NheI/HindIII fragment and the FtsZ extended C-terminal tail was cloned at an internal SacI site at the end of Gfp. FtsZ mutant proteins were constructed by site-directed mutagenesis of plasmids using the QuikChange II XL Site-Directed Mutagenesis Kit (Agilent).

To replace chromosomal $\operatorname{clpP}$ with $\operatorname{clpP}(S 97 A)$, we first used site-directed mutagenesis of the ClpP expression plasmid (pET-ClpP) to construct pET-ClpP(S97A), and then amplified $\operatorname{clp} P(S 97 A)$ using recombination primers containing homology to 40bp regions flanking the $c l p P$ locus. In the recipient strain, the $c l p P$ gene was deleted by lambda-Red recombination and replaced with a kanamycin cassette linked to the parE gene under the control of a rhamnose promoter ([두 $]$ and J. Teramoto, K. A. Datsenko, 
and B. L. Wanner, unpublished results). The amplified $\operatorname{clp} P(S 97 A)$ containing flanking sites for recombination was reinserted at the $c l p P$ locus and selected for by growth on Lrhamnose (1\%). Recombinants were confirmed by sequencing. Similarly, clpX, clpP and $\min C$ wild type genes were restored in deletion strains at their native loci (Table 1).

\section{Expression and purification of proteins}

ClpX, ClpP, FtsZ, and FtsZ(R379E) were each expressed in E. coli BL21 ( $\lambda$ DE3) and purified as described $[\underline{24}, \underline{25}, \underline{72}, \underline{73}]$. FtsZ(G105S), FtsZ(G105S, R379E), and FtsZ(3527A) were purified as wild type FtsZ. FtsZ wild type and mutant proteins were labeled with Alexa Fluor 488 or 647, where indicated, and then fluorescent active subunits were obtained by cycles of polymerization and depolymerization $[25,74]$. GfpFtsZ and Gfp- $Z_{\mathrm{C} 67}$ were overexpressed in E. coli $\mathrm{BL} 21(\lambda \mathrm{DE} 3)$ grown in Lennox broth to an $\mathrm{OD}_{600}$ of 1.0 at $37^{\circ} \mathrm{C}$ and then induced with $1 \mathrm{mM}$ IPTG for 3 hours at $30^{\circ} \mathrm{C}$. Cells were lysed by French press, and soluble lysate was bound to TALON superflow resin (GE Healthcare). Histidine-tagged proteins were eluted with an imidazole gradient and imidazole was removed by buffer exchange. Protein concentrations are reported as FtsZ monomers, ClpX hexamers and ClpP tetradecamers.

\section{Degradation assays}

Gfp-FtsZ and Gfp- $Z_{\mathrm{C} 67}$ were degraded by ClpXP in buffer containing $20 \mathrm{mM}$ HEPES pH 7.0, $150 \mathrm{mM} \mathrm{KCl}$, and $10 \mathrm{mM} \mathrm{MgCl}_{2}$ with $5 \mathrm{mM}$ ATP, $0.005 \%$ Triton $\mathrm{X}-100$, and $2 \mathrm{mM} \mathrm{GTP}$, where indicated, with acetate kinase $\left(25 \mu \mathrm{g} \mathrm{ml}^{-1}\right)$ and acetyl phosphate (15 mM) (bifunctional ATP/GTP regenerating system). Fluorescence was monitored with 
an Agilent Eclipse Spectrophotometer (excitation $395 \mathrm{~nm}$, emission $510 \mathrm{~nm}$ ). Degradation of FtsZ wild type and mutant proteins labeled with Alexa Fluor 488 or 647, where indicated, was performed as described by quantitating fluorescent peptides [25]. Hill coefficients were calculated by fitting the data to a nonlinear regression model using GraphPad Prism (version 6.0b) $\left[\mathrm{Y}=\mathrm{Rmax} * \mathrm{X}^{\wedge} \mathrm{h} /\left(\mathrm{K}^{\wedge} \mathrm{h}+\mathrm{X}^{\wedge} \mathrm{h}\right)\right.$, where $\mathrm{Rmax}$ is the maximum response, $\mathrm{K}$ is the concentration at half-maximal response and $\mathrm{h}$ is the Hill slope].

\section{Polymerization assays and GTP hydrolysis}

Gfp-FtsZ and/or FtsZ was incubated with or without GTP (2 mM) for $3 \mathrm{~min}$. in assembly buffer (50 mM MES, pH $6.5100 \mathrm{mM} \mathrm{KCl,} 10 \mathrm{mM} \mathrm{MgCl}_{2}$ ) in the presence of a regenerating system (same one used for degradation assays) and spun for $30 \mathrm{~min}$ at $23^{\circ} \mathrm{C}$ in a Beckman TLA 120.1 rotor at 129,000xg. Supernatants and pellets were collected in equivalent volumes of $1 \mathrm{x}$ lithium dodecyl sulfate (LDS) sample buffer (Life Technologies) and analyzed by SDS-PAGE. GTP hydrolysis of FtsZ wild type and mutant proteins was assayed using the Biomol Green phosphate detection reagent (Enzo Life Sciences) as described [25].

\section{Sucrose gradient fractionation}

Fluorescence-labeled FtsZ $(24 \mu \mathrm{M})$ was incubated with and without GTP (2 mM), acetate kinase $\left(25 \mu \mathrm{g} \mathrm{ml}^{-1}\right)$ and acetyl phosphate $(15 \mathrm{mM})$ in assembly buffer $[50 \mathrm{mM}$ MES (morpholino-ethane-sulfonic acid), $\mathrm{pH}$ 6.5, $100 \mathrm{mM} \mathrm{KCl,} 10 \mathrm{mM} \mathrm{MgCl}_{2}$ ] for $5 \mathrm{~min}$. ClpXP $(1.6 \mu \mathrm{M})$ and ATP $(5 \mathrm{mM})$ were added, where indicated, and all reactions were 
incubated for 90 min. Reaction products were crosslinked with dithiobis(succinimidyl propionate) (DSP) (0.6 mM). After $30 \mathrm{~min}$, reactions were quenched with Tris- $\mathrm{HCl}$ (25 $\mathrm{mM}, \mathrm{pH}$ 8), applied to a $5-20 \%$ sucrose gradient with a $40 \%$ sucrose cushion, and centrifuged for 180 min at $4^{\circ} \mathrm{C}$ at $100,000 \mathrm{xg}$ in a Beckman TLS-55 rotor. Fractions (20 $\mu l)$ were collected and analyzed by fluorescence.

\section{Microscopy}

Overnight cultures of MG1655 wild type and deletion strains expressing Gfptagged (Gfpuv) FtsZ fusion proteins were diluted 1:50 into fresh media containing ampicillin $\left(100 \mu \mathrm{g} \mathrm{ml}^{-1}\right)$ and arabinose $(70 \mu \mathrm{M}$ or $140 \mu \mathrm{M}$, where indicated), and then grown for three hours at $30^{\circ} \mathrm{C}$. Cells were directly applied to a $4 \%$ agarose pad containing MOPS [3-( $N$-morpholino) propanesulfonic acid] minimal media with $0.5 \%$ glycerol and a coverslip was added. Images were collected with a Zeiss LSM 700 confocal fluorescence microscope and images were captured on an AxioCam digital camera with ZEN 2012 software. In fluorescence recovery assays, regions of each Z-ring were selected and bleached at full laser power for one iteration until the initial fluorescence was reduced by at least $40 \%$. Recovery images were captured at 3, 6 or $8 \mathrm{sec}$ intervals, where indicated. The fluorescence intensity of the region at each interval was quantified using NIH ImageJ. Intensity values were normalized to the recovery period maximum plateau value, which was on average $70-80 \%$ of the initial pre-bleach fluorescence. Recovery was plotted as fluorescence intensity with time and fit to a nonlinear regression model $\{\mathrm{Y}=\mathrm{Y} 0$ $+($ Plateau-Y0)*[1-exp(-K*x)], where $\mathrm{Y} 0$ is the $\mathrm{Y}$-value at time zero, Plateau is the $\mathrm{Y}$ value at infinite time and $\mathrm{K}$ is the rate constant $\}$ using GraphPad Prism (version 6.0b). 
Half-time recovery values were calculated for individual replicates and then averaged. The percentage of Z-ring fluorescence per cell was measured in ImageJ for at least 10 cells.

\section{Immunoblotting}

Cells were grown as described and total proteins were precipitated with trichloroacetic acid $(15 \% \mathrm{v} / \mathrm{v})$. Proteins were resuspended in buffer containing $2 \%$ SDS, quantified by the bicinchoninic acid assay, and analyzed by SDS-PAGE and immunoblotting using antibodies to Gfp (Thermo Scientific), FtsZ, ClpX, ClpP or MinC as described $[\underline{24}, \underline{45}]$.

\section{Acknowledgements}

We thank Shannon May, Erin Pelton and Eric DiBiasio for helpful discussions, Anthony Velasquez for technical assistance, Barry Wanner for pKD267, Piet de Boer for $\lambda \mathrm{CH} 151$, Matt Chenoweth for strain construction, and Paul Johnson and Janet Atoyan for microscopy and sequencing assistance. Sequencing and microscopy were performed at the Rhode Island Genomics and Sequencing Center, which is supported in part by the National Science Foundation under EPSCoR Grants Nos. 0554548 \& EPS-1004057. 


\section{References}

1. Egan AJ, Vollmer W (2013) The physiology of bacterial cell division. Ann N Y Acad Sci. 1277: 8-28. 10.1111/j.1749-6632.2012.06818.x

2. Erickson HP, Anderson DE, Osawa M (2010) FtsZ in bacterial cytokinesis: cytoskeleton and force generator all in one. Microbiol Mol Biol Rev. 74: 504-528. 10.1128/MMBR.00021-10

3. Oliva MA, Cordell SC, Lowe J (2004) Structural insights into FtsZ protofilament formation. Nat Struct Mol Biol. 11: 1243-1250. 10.1038/nsmb855

4. Ortiz C, Natale P, Cueto L, Vicente M (2016) The keepers of the ring: regulators of FtsZ assembly. FEMS Microbiol Rev. 40: 57-67. 10.1093/femsre/fuv040

5. Fu G, Huang T, Buss J, Coltharp C, Hensel Z, et al. (2010) In vivo structure of the $E$. coli FtsZ-ring revealed by photoactivated localization microscopy (PALM). PloS One. 5: e12682. 10.1371/journal.pone.0012680

6. Szwedziak P, Wang Q, Bharat TA, Tsim M, Lowe J (2014) Architecture of the ring formed by the tubulin homologue FtsZ in bacterial cell division. eLife. 3: e04601. 10.7554/eLife.04601

7. Strauss MP, Liew AT, Turnbull L, Whitchurch CB, Monahan LG, Harry EJ (2012) 3DSIM super resolution microscopy reveals a bead-like arrangement for FtsZ and the division machinery: implications for triggering cytokinesis. PLoS Biol. 10: e1001389. 10.1371/journal.pbio.1001389

8. Li Z, Trimble MJ, Brun YV, Jensen GJ (2007) The structure of FtsZ filaments in vivo 
suggests a force-generating role in cell division. EMBO J. 26: 4694-4708.

9. Holden SJ, Pengo T, Meibom KL, Fernandez Fernandez C, Collier J, Manley S (2014) High throughput 3D super-resolution microscopy reveals Caulobacter crescentus in vivo Z-ring organization. Proc Natl Acad Sci U S A. 111: 4566-4571. $10.1073 /$ pnas. 1313368111

10. Jacq M, Adam V, Bourgeois D, Moriscot C, Di Guilmi AM, Vernet T, et al. (2015) Remodeling of the Z-Ring Nanostructure during the Streptococcus pneumoniae Cell Cycle Revealed by Photoactivated Localization Microscopy. mBio. 6. 10.1128/mBio.01108-15

11. Buss J, Coltharp C, Huang T, Pohlmeyer C, Wang SC, Hatem C, et al. (2013) In vivo organization of the FtsZ-ring by ZapA and ZapB revealed by quantitative superresolution microscopy. Mol Microbiol. 89: 1099-1120. 10.1111/mmi.12331

12. Anderson DE, Gueiros-Filho FJ, Erickson HP (2004) Assembly dynamics of FtsZ rings in Bacillus subtilis and Escherichia coli and effects of FtsZ-regulating proteins. J Bacteriol. 186: 5775-5781.

13. Stricker J, Maddox P, Salmon ED, Erickson HP (2002) Rapid assembly dynamics of the Escherichia coli FtsZ-ring demonstrated by fluorescence recovery after photobleaching. Proc Natl Acad Sci U S A. 99: 3171-3175.

14. RayChaudhuri D, Park JT (1992) Escherichia coli cell-division gene ftsZ encodes a novel GTP-binding protein. Nature. 359: 251-254. 10.1038/359251a0

15. Dai K, Xu Y, Lutkenhaus J (1993) Cloning and characterization of $f t s N$, an essential cell division gene in Escherichia coli isolated as a multicopy suppressor of 
ftsA12(Ts). J Bacteriol. 175: 3790-3797.

16. de Boer P, Crossley R, Rothfield L (1992) The essential bacterial cell-division protein FtsZ is a GTPase. Nature. 359: 254-256. 10.1038/359254a0

17. Buss J, Coltharp C, Shtengel G, Yang X, Hess H, Xiao J (2015) A multi-layered protein network stabilizes the Escherichia coli FtsZ-ring and modulates constriction dynamics. PLoS Genetics. 11: e1005128. 10.1371/journal.pgen.1005128

18. Galli E, Gerdes K (2012) FtsZ-ZapA-ZapB interactome of Escherichia coli. J Bacteriol. 194: 292-302. 10.1128/JB.05821-11

19. Small E, Marrington R, Rodger A, Scott DJ, Sloan K, Roper D, et al. (2007) FtsZ polymer-bundling by the Escherichia coli ZapA orthologue, YgfE, involves a conformational change in bound GTP. J Mol Biol. 369: 210-221. 10.1016/j.jmb.2007.03.025

20. Shen B, Lutkenhaus J (2009) The conserved C-terminal tail of FtsZ is required for the septal localization and division inhibitory activity of $\operatorname{MinC}(\mathrm{C}) / \mathrm{MinD}$. Mol Microbiol. 72: 410-424.

21. Shen B, Lutkenhaus J (2010) Examination of the interaction between FtsZ and MinCN in E. coli suggests how MinC disrupts Z rings. Mol Microbiol. 75: 12851298.

22. Cho H, McManus HR, Dove SL, Bernhardt TG (2011) Nucleoid occlusion factor SlmA is a DNA-activated FtsZ polymerization antagonist. Proc Natl Acad Sci U S A. 108: $3773-3778$. 
23. Tonthat NK, Arold ST, Pickering BF, Van Dyke MW, Liang S, Lu Y, et al. (2011) Molecular mechanism by which the nucleoid occlusion factor, SlmA, keeps cytokinesis in check. EMBO J. 30: 154-164.

24. Camberg JL, Hoskins JR, Wickner S (2009) ClpXP protease degrades the cytoskeletal protein, FtsZ, and modulates FtsZ polymer dynamics. Proc Natl Acad Sci U S A. 106: 10614-10619.

25. Camberg JL, Viola MG, Rea L, Hoskins JR, Wickner S (2014) Location of dual sites in E. coli FtsZ important for degradation by ClpXP; one at the C-terminus and one in the disordered linker. PloS One. 9: e94964. 10.1371/journal.pone.0094964

26. Marteyn BS, Karimova G, Fenton AK, Gazi AD, West N, Touqui L, et al. (2014) ZapE is a novel cell division protein interacting with FtsZ and modulating the Zring dynamics. mBio. 5: e00022-00014. 10.1128/mBio.00022-14

27. Shih YL, Zheng M (2013) Spatial control of the cell division site by the Min system in Escherichia coli. Environ Microbiol. 15: 3229-3239. 10.1111/1462-2920.12119

28. Baker TA, Sauer RT (2012) ClpXP, an ATP-powered unfolding and proteindegradation machine. Biochim Biophys Acta. 1823: 15-28. 10.1016/j.bbamcr.2011.06.007

29. Smith SC, Joshi KK, Zik JJ, Trinh K, Kamajaya A, Chien P, et al. (2014) Cell cycledependent adaptor complex for ClpXP-mediated proteolysis directly integrates phosphorylation and second messenger signals. Proc Natl Acad Sci U S A. 111: 14229-14234. 10.1073/pnas.1407862111

30. Williams B, Bhat N, Chien P, Shapiro L (2014) ClpXP and ClpAP proteolytic activity 
on divisome substrates is differentially regulated following the Caulobacter asymmetric cell division. Mol Microbiol. 93: 853-866. 10.1111/mmi.12698

31. Neher SB, Villen J, Oakes EC, Bakalarski CE, Sauer RT, Gygi SP, et al. (2006) Proteomic profiling of ClpXP substrates after DNA damage reveals extensive instability within SOS regulon. Mol Cell. 22: 193-204.

32. Flynn JM, Neher SB, Kim YI, Sauer RT, Baker TA (2003) Proteomic discovery of cellular substrates of the ClpXP protease reveals five classes of ClpX-recognition signals. Mol Cell. 11: 671-683.

33. Haeusser DP, Lee AH, Weart RB, Levin PA (2009) ClpX inhibits FtsZ assembly in a manner that does not require its ATP hydrolysis-dependent chaperone activity. $\mathrm{J}$ Bacteriol. 191: 1986-1991.

34. Weart RB, Nakano S, Lane BE, Zuber P, Levin PA (2005) The ClpX chaperone modulates assembly of the tubulin-like protein FtsZ. Mol Microbiol. 57: 238-249.

35. Dziedzic R, Kiran M, Plocinski P, Ziolkiewicz M, Brzostek A, Moomey M, et al. (2010) Mycobacterium tuberculosis ClpX Interacts with FtsZ and Interferes with FtsZ Assembly. PloS One. 5: e11058.

36. Sugimoto S, Yamanaka K, Nishikori S, Miyagi A, Ando T, Ogura T (2010) AAA+ chaperone $\mathrm{ClpX}$ regulates dynamics of prokaryotic cytoskeletal protein FtsZ. The J Biol Chem. 285: 6648-6657.

37. Roll-Mecak A, Vale RD (2008) Structural basis of microtubule severing by the hereditary spastic paraplegia protein spastin. Nature. 451: 363-367.

38. Roll-Mecak A, Vale RD (2006) Making more microtubules by severing: a common 
theme of noncentrosomal microtubule arrays? J Cell Biol. 175: 849-851.

39. Eckert T, Le DT, Link S, Friedmann L, Woehlke G (2012) Spastin's microtubulebinding properties and comparison to katanin. PloS One 7: e50161. 10.1371/journal.pone.0050161

40. Monroe N, Hill CP (2016) Meiotic Clade AAA ATPases: Protein Polymer Disassembly Machines. J Mol Biol. 428: 1897-1911. 10.1016/j.jmb.2015.11.004

41. Sharma N, Bryant J, Wloga D, Donaldson R, Davis RC, Jerka-Dziadosz M, et al. (2007) Katanin regulates dynamics of microtubules and biogenesis of motile cilia. J Cell Biol. 178: 1065-1079. 10.1083/jcb.200704021

42. Du S, Park KT, Lutkenhaus J (2015) Oligomerization of FtsZ converts the FtsZ tail motif (conserved carboxy-terminal peptide) into a multivalent ligand with high avidity for partners ZipA and SlmA. Mol Microbiol. 95: 173-188. $10.1111 / \mathrm{mmi} .12854$

43. Khlebnikov A, Datsenko KA, Skaug T, Wanner BL, Keasling JD (2001) Homogeneous expression of the $\mathrm{P}(\mathrm{BAD})$ promoter in Escherichia coli by constitutive expression of the low-affinity high-capacity AraE transporter. Microbiology. 147: 3241-3247.

44. Blattner FR, Plunkett G, 3rd, Bloch CA, Perna NT, Burland V, Riley M, et al. (1997) The complete genome sequence of Escherichia coli K-12. Science. 277: 14531462.

45. Camberg JL, Hoskins JR, Wickner S (2011) The interplay of ClpXP with the cell division machinery in Escherichia coli. J Bacteriol. 193: 1911-1918. 
46. Baba T, Ara T, Hasegawa M, Takai Y, Okumura Y, Baba M, et al. (2006) Construction of Escherichia coli K-12 in-frame, single-gene knockout mutants: the Keio collection. Mol Sys Biol. 2: 20060008.

47. Bernhardt TG, de Boer PA (2004) Screening for synthetic lethal mutants in Escherichia coli and identification of EnvC (YibP) as a periplasmic septal ring factor with murein hydrolase activity. Mol Microbiol. 52: 1255-1269.

48. Cherepanov PP, Wackernagel W (1995) Gene disruption in Escherichia coli: TcR and KmR cassettes with the option of Flp-catalyzed excision of the antibioticresistance determinant. Gene. 158: 9-14.

49. Maurizi MR, Clark WP, Kim SH, Gottesman S (1990) ClpP represents a unique family of serine proteases. J Biol Chem. 265: 12546-12552.

50. Datsenko KA, Wanner BL (2000) One-step inactivation of chromosomal genes in Escherichia coli K-12 using PCR products. Proc Natl Acad Sci U S A. 97: 66406645. 10.1073/pnas. 120163297

51. Bramhill D, Thompson CM (1994) GTP-dependent polymerization of Escherichia coli FtsZ protein to form tubules. Proc Natl Acad Sci U S A. 91: 5813-5817.

52. Lu C, Stricker J, Erickson HP (2001) Site-specific mutations of FtsZ--effects on GTPase and in vitro assembly. BMC Microbiol. 1: 7.

53. Redick SD, Stricker J, Briscoe G, Erickson HP (2005) Mutants of FtsZ targeting the protofilament interface: effects on cell division and GTPase activity. J Bacteriol. 187: 2727-2736. 10.1128/JB.187.8.2727-2736.2005

54. Pazos M, Natale P, Vicente M (2012) A specific role for the ZipA protein in cell 
division: stabilization of the FtsZ protein. J Biol Chem. 288: 3219-3226. 10.1074/jbc.M112.434944

55. Bernhardt TG, de Boer PA (2005) SlmA, a nucleoid-associated, FtsZ binding protein required for blocking septal ring assembly over chromosomes in E. coli. Molecular cell 18: 555-564.

56. Yu XC, Margolin W (1999) FtsZ ring clusters in min and partition mutants: role of both the Min system and the nucleoid in regulating FtsZ ring localization. Mol Microbiol. 32: 315-326.

57. Teather RM, Collins JF, Donachie WD (1974) Quantal behavior of a diffusible factor which initiates septum formation at potential division sites in Escherichia coli. J Bacteriol. 118: 407-413.

58. Hale CA, de Boer PA (1999) Recruitment of ZipA to the septal ring of Escherichia coli is dependent on FtsZ and independent of FtsA. J Bacteriol. 181: 167-176.

59. Buczek MS, Cardenas Arevalo AL, Janakiraman A (2016) ClpXP and ClpAP control the Escherichia coli division protein ZapC by proteolysis. Microbiology. 162: 909-920. 10.1099/mic.0.000278

60. Geissler B, Shiomi D, Margolin W (2007) The ftsA* gain-of-function allele of Escherichia coli and its effects on the stability and dynamics of the $Z$ ring. Microbiology. 153: 814-825. 10.1099/mic.0.2006/001834-0

61. Soderstrom B, Mirzadeh K, Toddo S, von Heijne G, Skoglund U, Daley DO (2016) Coordinated disassembly of the divisome complex in Escherichia coli. Mol Microbiol. 10.1111/mmi.13400 
62. Soderstrom B, Skoog K, Blom H, Weiss DS, von Heijne G, Daley DO (2014) Disassembly of the divisome in Escherichia coli: evidence that FtsZ dissociates before compartmentalization. Mol Microbiol. 92: 1-9. 10.1111/mmi.12534

63. Adams DW, Errington J (2009) Bacterial cell division: assembly, maintenance and disassembly of the $\mathrm{Z}$ ring. Nat Rev Microbiol. 7: 642-653.

64. Arjes HA, Lai B, Emelue E, Steinbach A, Levin PA (2015) Mutations in the bacterial cell division protein FtsZ highlight the role of GTP binding and longitudinal subunit interactions in assembly and function. BMC Microbiol. 15: 209. $10.1186 / \mathrm{s} 12866-015-0544-\mathrm{Z}$

65. Ling L, Montano SP, Sauer RT, Rice PA, Baker TA (2015) Deciphering the Roles of Multicomponent Recognition Signals by the AAA+ Unfoldase ClpX. J Mol Biol. 427: 2966-2982. 10.1016/j.jmb.2015.03.008

66. Buske PJ, Levin PA (2012) Extreme C terminus of bacterial cytoskeletal protein FtsZ plays fundamental role in assembly independent of modulatory proteins. J Biol Chem. 287: 10945-10957. 10.1074/jbc.M111.330324

67. Hale CA, de Boer PA (1997) Direct binding of FtsZ to ZipA, an essential component of the septal ring structure that mediates cell division in E. coli. Cell 88: 175-185.

68. Pichoff S, Lutkenhaus J (2002) Unique and overlapping roles for ZipA and FtsA in septal ring assembly in Escherichia coli. EMBO J. 21: 685-693.

69. Liu Z, Mukherjee A, Lutkenhaus J (1999) Recruitment of ZipA to the division site by interaction with FtsZ. Mol Microbiol. 31: 1853-1861.

70. Szwedziak P, Wang Q, Freund SM, Lowe J (2012) FtsA forms actin-like 
protofilaments. EMBO J. 31: 2249-2260. 10.1038/emboj.2012.76

71. Ma X, Margolin W (1999) Genetic and functional analyses of the conserved Cterminal core domain of Escherichia coli FtsZ. J Bacteriol. 181: 7531-7544.

72. Grimaud R, Kessel M, Beuron F, Steven AC, Maurizi MR (1998) Enzymatic and structural similarities between the Escherichia coli ATP- dependent proteases, ClpXP and ClpAP. J Biol Chem. 273: 12476-12481.

73. Maurizi MR, Thompson MW, Singh SK, Kim SH (1994) Endopeptidase Clp: ATPdependent Clp protease from Escherichia coli. Methods Enzymol. 244: 314-331.

74. Gonzalez JM, Jimenez M, Velez M, Mingorance J, Andreu JM, Vicente M, et al. (2003) Essential cell division protein FtsZ assembles into one monomer-thick ribbons under conditions resembling the crowded intracellular environment. J Biol Chem. 278: 37664-37671. 
TABLE 1. E. coli strains and plasmids.

\begin{tabular}{|c|c|c|}
\hline $\begin{array}{l}\text { Strain } \\
\text { Plasmid }\end{array}$ & Genotype & $\begin{array}{l}\text { Source, } \\
\text { reference or } \\
\text { Construction }{ }^{a, b} \\
, c\end{array}$ \\
\hline \multicolumn{3}{|l|}{ Strains } \\
\hline MG1655 & LAM-rph-1 & [44] \\
\hline BW27750 & $\begin{array}{l}\text { lac/9rrnB3 } \Delta / a c Z 4787 \quad h s d R 514 \\
\Delta(\text { araBAD) } 567 \quad \Delta(\text { rhaBAD)568 } \\
\Delta\left(\text { araFGH) } \phi\left(\Delta a r a E p:: k a n \mathrm{P}_{\mathrm{CP} 18-a r a E)}\right.\right.\end{array}$ & [43] \\
\hline BW27784 & 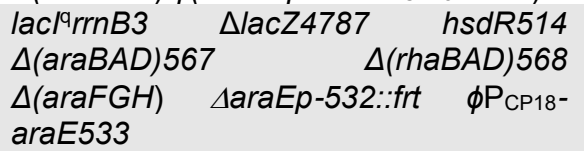 & [43] \\
\hline JC0390 & MG1655 (4araEp::kan P $\mathrm{CP}_{18-a r a E)}$ & $\begin{array}{l}\text { P1(BW27750) } \\
\text { MG1655 }\end{array}$ \\
\hline JC0291 & MG1655 $\Delta c / p X:: f r t$ & pCP20 \\
\hline JC0394 & $\begin{array}{l}\text { MG1655 } \Delta c l p X:: f r t, \text { araEp::kan PCP18- } \\
\text { araE }\end{array}$ & $\begin{array}{l}\text { P1(BW27750) } \\
\text { JC0291 }\end{array}$ \\
\hline MV03720 & MG1655 $\Delta c / p X:: k a n-p a r E$ & pKD267 $7^{c} ; \lambda \operatorname{Red}$ \\
\hline MV03721 & MG1655 clpX-restored & MV03720; $\lambda$ Red \\
\hline MV03722 & $\begin{array}{l}\text { MG1655 clpX-restored, } \triangle a r a E p:: k a n \\
\text { P }_{\mathrm{CP} 18-a r a E}\end{array}$ & MV03721 \\
\hline MV0050 & MG1655 $\Delta c / p P:: f r t$ & pCP20 \\
\hline MV0210 & $\begin{array}{l}\text { MG1655 } \Delta c / p P:: \text { frt, } \Delta a r a E p:: k a n P_{\mathrm{CP} 18^{-}} \\
\operatorname{araE}\end{array}$ & $\begin{array}{l}\text { P1(BW27750) } \\
\text { MV0050 }\end{array}$ \\
\hline MV0242 & MG1655 $\Delta c / p P:: k a n-p a r E$ & \\
\hline MV0251 & MG1655 clpP(S97A) & MV0242; $\lambda$ Red \\
\hline MV0256 & $\begin{array}{l}\text { MG1655 clpP(S97A), daraE::kan } \mathrm{P}_{\mathrm{CP} 18^{-}} \\
\text {araE }\end{array}$ & $\begin{array}{l}\text { P1(BW27750) } \\
\text { MV0251 }\end{array}$ \\
\hline MV03711 & MG1655 clpP-restored & MV0242; $\lambda$ Red \\
\hline MV03712 & $\begin{array}{l}\text { MG1655 clpP-restored, } \Delta a r a E p:: k a n \\
\text { P }_{\mathrm{CP} 18-a r a E}\end{array}$ & MV03711 \\
\hline JC0395 & $\begin{array}{l}\text { MG1655 } \Delta \text { minC::frt, } \triangle a r a E p:: k a n P_{C P 18-} \\
\text { araE }\end{array}$ & $\begin{array}{l}\text { P1(BW27750) } \quad x \\
\text { JC0232 [45] }\end{array}$ \\
\hline MV0196 & MG1655 $\Delta s / m A:: f r t$ & $\begin{array}{l}\text { JC0232 [45] } \\
\text { P1(JW5641) [46] x } \\
\text { MG1655; pCP20 }\end{array}$ \\
\hline MV03730 & MG1655 $\Delta$ minC::kan-parE & pKD267c; $\lambda$ Red \\
\hline MV03731 & MG1655 minC-restored & MV03730; $\lambda$ Red \\
\hline MV03732 & $\begin{array}{l}\text { MG1655 minC-restored, } \Delta a r a E p:: k a n \\
\text { P }_{\text {CP18-araE }}\end{array}$ & MV03731 \\
\hline MV0198 & $\begin{array}{l}\text { MG1655 } \Delta s / m A:: f r t, \text { aaraEp::kan } \mathrm{P}_{\mathrm{CP} 18-} \\
\operatorname{araE}\end{array}$ & MV0196 \\
\hline MV0340 & MG1655 $\Delta z a p E:: f r t$ & $\begin{array}{l}\text { P1(JW3201) [46] x } \\
\text { MG1655; pCP20 }\end{array}$ \\
\hline MV0277 & $\begin{array}{l}\text { MG1655 } \Delta \text { zapE::frt, } \Delta a r a E p:: k a n \mathrm{P}_{\mathrm{CP} 18^{-}} \\
\text {araE }\end{array}$ & $\begin{array}{l}\text { P1(BW27750) } \\
\text { MV0340 }\end{array}$ \\
\hline MC181 & BW27784 $\lambda \mathrm{CH} 151$ [Plac::zipA-gfp] & $\lambda \mathrm{CH} 151[47]$ \\
\hline MV0226 & $\begin{array}{l}\text { BW27784 } \\
\lambda \mathrm{CH} 151\left[\mathrm{P}_{\text {lac::ZipA-gfp] }}\right.\end{array}$ & $\begin{array}{l}\text { P1(JC0259) [45] X } \\
\text { MC181 }\end{array}$ \\
\hline \multicolumn{3}{|l|}{ Plasmids } \\
\hline pET-H6-Gfp-FtsZ & kan & This study \\
\hline pET-H6-Gfp-Z 667 & kan & This study \\
\hline pET-H6-Gfp- & kan & This study \\
\hline
\end{tabular}




\begin{tabular}{|c|c|c|}
\hline$Z_{\mathrm{C} 67}(\mathrm{R} 379 \mathrm{E})$ & & \\
\hline pCP20 & amp flp recombinase & [48] \\
\hline pKD267 & kan Prham-parE & B. Wanner ${ }^{c}$ \\
\hline pET-ClpP & kan & [24] \\
\hline pET-ClpP(S97A) & kan & This study \\
\hline pET-FtsZ & kan & [24] \\
\hline pET-FtsZ(G105S) & kan & This study \\
\hline pET-FtsZ(R379E) & kan & [25] \\
\hline $\begin{array}{l}\text { pET- } \\
\text { FtsZ(G105S,R379 } \\
\text { E) }\end{array}$ & kan & This study \\
\hline pET-FtsZ(3527A) & kan & This study \\
\hline pET-ClpX & kan & [24] \\
\hline pGfp-FtsZ & amp Para::gfp-ftsZ & [45] \\
\hline pGfp-FtsZ(G105S) & amp Para:::gfp-ftsZ(G105S) & This study \\
\hline pGfp-FtsZ(R379E) & amp Para::gfp-ftsZ(R379E) & [25] \\
\hline $\begin{array}{l}\text { pGfp-FtsZ(G105S, } \\
\text { R379E) }\end{array}$ & amp Para::gfp-ftsZ(G105S, R379E) & This study \\
\hline pGfp-FtsZ(3527A) & amp Para:: :gfp-ftsZ(3527A) & This study \\
\hline
\end{tabular}


S1 TABLE. Cell lengths and fluorescence recovery times.

\begin{tabular}{|c|c|c|c|c|}
\hline Strain or plasmid & $\begin{array}{l}\text { Relevant } \\
\text { Genotype }\end{array}$ & $\begin{array}{l}\text { Average cell } \\
\text { length }^{\mathrm{a}}(\mu \mathrm{m})\end{array}$ & 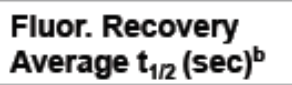 & $p$ value ${ }^{c}$ \\
\hline \multicolumn{5}{|l|}{ Host Strain + pGfp-FtsZ } \\
\hline JC0390 & wild type & $3.5 \pm 0.1$ & $6.2 \pm 0.5 \quad(n=18)$ & - \\
\hline JC0394 & $\Delta c / p X$ & $3.1 \pm 0.1$ & $10.6 \pm 1.3 \quad(n=38)$ & 0.0037 \\
\hline MV0210 & $\Delta c / p P$ & $3.0 \pm 0.1$ & $10.6 \pm 0.7 \quad(n=27)$ & $<0.0001$ \\
\hline MV0256 & $\operatorname{clpP}(S 97 A)$ & $2.7 \pm 0.04$ & $10.4 \pm 1.4 \quad(n=30)$ & 0.0072 \\
\hline JC0395 & $\Delta \min C$ & $6.1 \pm 0.2$ & $10.3 \pm 1.2 \quad(n=20)$ & 0.0049 \\
\hline MV0198 & $\Delta s / m A$ & $4.4 \pm 0.2$ & $11.2 \pm 1.7 \quad(n=22)$ & 0.0091 \\
\hline MV0277 & $\Delta \mathrm{zapE}$ & $4.3 \pm 0.1$ & $9.2 \pm 0.8 \quad(n=18)$ & 0.0048 \\
\hline MV03722 & clpX-restored & n.d. & $8.2 \pm 1.4 \quad(n=8)$ & n.s. \\
\hline MV03712 & clpP-restored & n.d. & $8.1 \pm 0.6 \quad(n=11)$ & n.s. \\
\hline MV03732 & minc-restored & n.d. & $6.7 \pm 1.1 \quad(n=9)$ & n.s. \\
\hline \multicolumn{5}{|l|}{ MG1655 (JC0390) + plasmid } \\
\hline pGfp-FtsZ(G105S) & & $4.0 \pm 0.1$ & $12.6 \pm 1.5 \quad(n=25)$ & 0.0003 \\
\hline pGfp-FtsZ(R379E) & & $9.6 \pm 0.6$ & $12.1 \pm 1.6 \quad(n=26)$ & 0.0013 \\
\hline pGfp-FtsZ $\left(352_{7 A}\right)$ & & n.d. & $8.2 \pm 1.1 \quad(n=18)$ & n.s. \\
\hline pGfp-FtsZ(G105S, R379E) & & $>10 \mu \mathrm{m}$ & $13.8 \pm 2.3 \quad(n=18)$ & 0.0041 \\
\hline \multicolumn{5}{|l|}{ BW27784 zipA-gfp } \\
\hline MC181 & & $5.4 \pm 0.1$ & $5.8 \pm 0.6 \quad(n=21)$ & \\
\hline MV0226 & $\Delta c / p X$ & $4.3 \pm 0.1$ & $5.8 \pm 0.5 \quad(n=23)$ & \\
\hline
\end{tabular}

${ }^{a}$ Average cell length was calculated by measuring a minimum of 200 cells; 'n.d.' is not determined.

${ }^{b}$ Error values are reported as SEM.

${ }^{c} p$ values for each data set compared to wild type strain JC0390 expressing Gfp-FtsZ were calculated by Welch's t test . A $p$ value $>0.01$ was not significant (n.s.). 


\section{Fig 1. Degradation and localization of Gfp-tagged FtsZ chimeras.}

(A) Schematic of native FtsZ, Gfp-FtsZ, and Gfp- $Z_{\text {C67 }}$ showing position of Gfp and FtsZ polymerization domain (1-316), unstructured linker (317-369) and C-terminal (370-383) regions. Sites important for ClpXP degradation are shown (degradation site-1, 379-383; degradation site-2, 352-358). (B) Gfp-Z $Z_{\mathrm{C} 67}(3 \mu \mathrm{M})$ degradation was measured by monitoring loss of fluorescence with time in the absence (white circles) and presence (black circles) of ClpXP $(1 \mu \mathrm{M})$, ATP $(5 \mathrm{mM})$ and an ATP regenerating system. The curves shown are representative of at least three replicates. (C) Gfp-FtsZ (5 $\mu \mathrm{M})$ degradation was measured by monitoring loss of fluorescence with time in the presence of ClpXP $(1 \mu \mathrm{M})$, ATP $(5 \mathrm{mM})$ and a regenerating system in the presence (black triangles) or absence (white triangles) of GTP $(2 \mathrm{mM})$, where indicated. Gfp-FtsZ $(5 \mu \mathrm{M})$ fluorescence was also measured in the absence of ClpXP (white circles). The curves shown are representative of at least three replicates. (D) Sedimentation of FtsZ $(10 \mu \mathrm{M})$ and Gfp-FtsZ $(10 \mu \mathrm{M})$ polymers with GTP, or using different ratios of Gfp-FtsZ to FtsZ (total of $10 \mu \mathrm{M}$ per reaction), collected by ultracentrifugation. Pellet fractions containing FtsZ polymers and soluble fractions containing non-polymerized FtsZ are shown. (E) Fluorescence microscopy and DIC images of wild type MG1655-derived cells (JC0390) in $\log$ phase expressing Gfp-FtsZ induced with $70 \mu \mathrm{M}$ arabinose. (F) Expression of plasmid encoded Gfp-FtsZ and chromosome encoded FtsZ from cell extracts $(1 \mu \mathrm{g}$ total protein) described in E using antibodies to FtsZ and Gfp. (G) Fluorescence intensity across the long axis of the cell was measured and plotted to determine the relative position of the Z-ring. Inset shows the fluorescence image used for quantitation. 
Individual cells were chosen as representative of the population. $(\mathrm{H})$ Box and whiskers plot showing total fluorescence at the Z-ring and total cell fluorescence for wild type cells (JC0390) expressing Gfp-FtsZ ( $\mathrm{n}=11)$. The extent of the box encompasses the interquartile range of the fluorescence intensity, whiskers extend to the maximum and minimum fluorescence intensities, and the line within each box represents the median. 
A

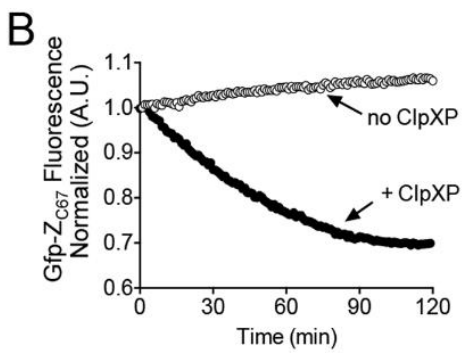

C
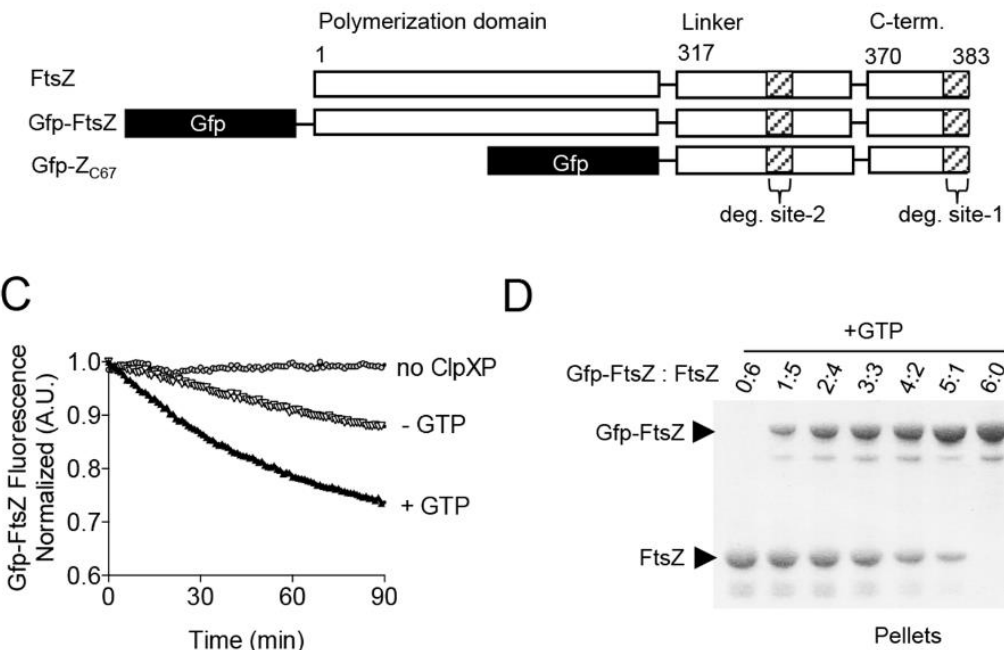

D

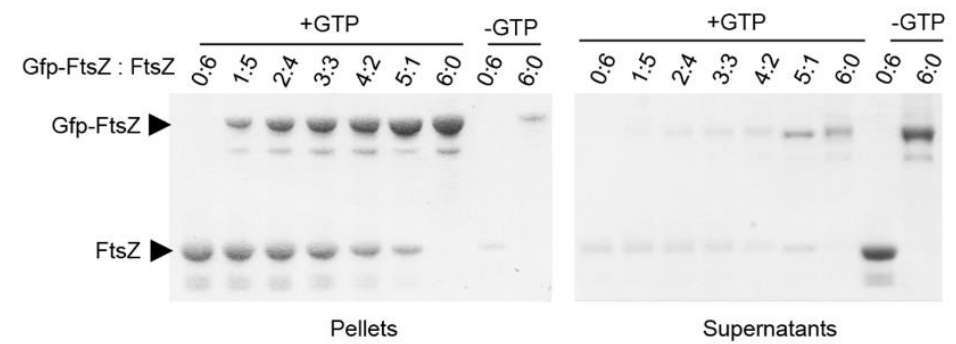

E

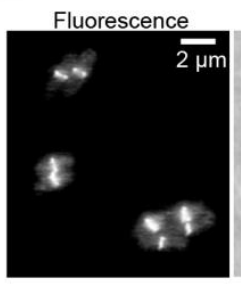

DIC

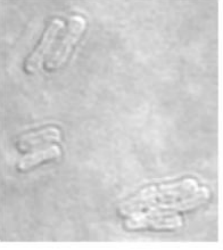

F

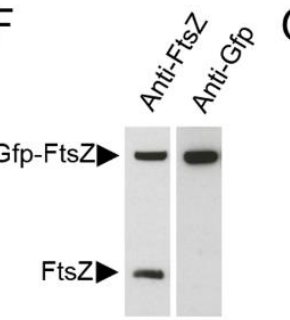

$\mathrm{H}$

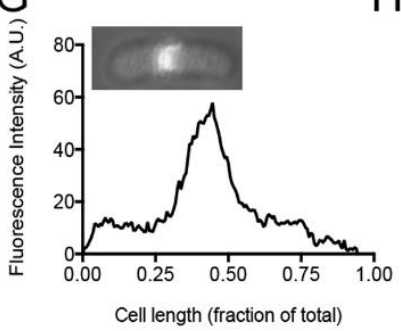

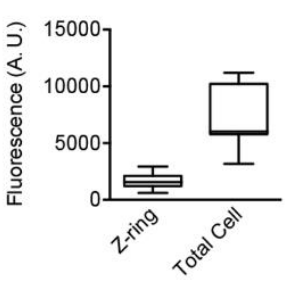


Fig 2. Photobleaching and recovery of the Z-ring in wild type strain.

(A) A region of the Z-ring was selected and bleached from wild type cells (JC0390) expressing Gfp-FtsZ and grown in LB containing arabinose $(70 \mu \mathrm{M})$ as described. Fluorescence recovery in the selected region was monitored every $8 \mathrm{sec}$ for $72 \mathrm{sec}$ (B) and plotted with time. (C) Box and whiskers plot of recovery half-times for Z-rings in wild type cells with various recovery intervals $(8 \mathrm{sec}, 3 \mathrm{sec}$, and $6 \mathrm{sec})(\mathrm{n} \geq 16)$. The extent of the box encompasses the interquartile range of the fluorescence recovery halftimes, and whiskers extend to the maximum and minimum values. The line within each box represents the median, with the mean value indicated by ' + '. 

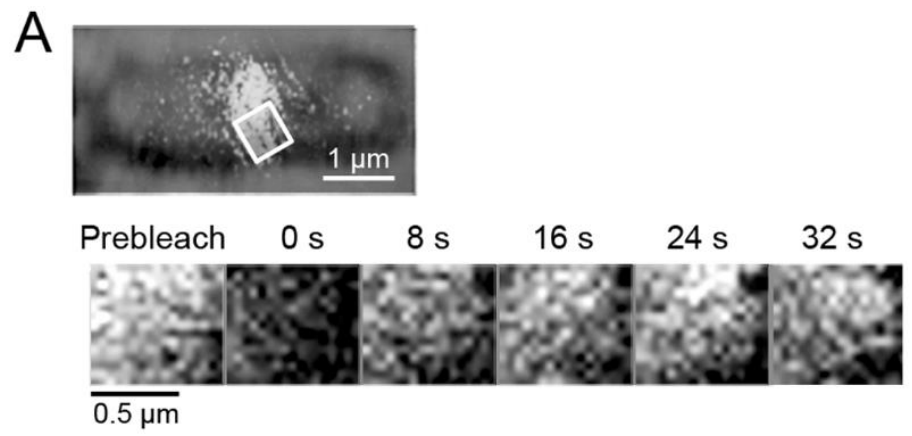

B

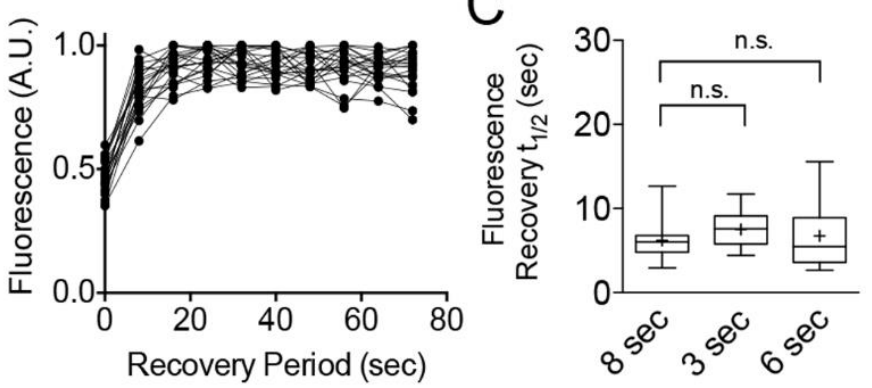


Fig 3. Z-ring assembly and dynamics in clp deficient strains. Fluorescence intensity across the long axis of the cell was measured and plotted for cells deleted for $\operatorname{clp} X$ (JC0394) (A), clpP (MV0210) (B), and cells with chromosomal clpP(S97A) (MV0256) (C). Insets show the fluorescence image used for quantitation. Individual cells were chosen as representative of the population. (D) Expression of $\mathrm{ClpP}$ and $\mathrm{ClpP}(\mathrm{S} 97 \mathrm{~A})$ from wild type (JC0390), $\Delta c l p P$ (MV0210), and $\operatorname{clpP}(S 97 A)$ (MV0256) cell extracts (40 $\mu \mathrm{g}$ total protein) using antibodies to ClpP. (E) Box and whiskers plot of recovery half-times for Z-rings in wild type cells (JC0390) and cells deleted for clpX (JC0394), clpP (MV0210), and containing chromosomal clpP(S97A) (MV0256). The extent of the box encompasses the interquartile range of the fluorescence recovery half-times, and whiskers extend to the maximum and minimum values. The line within each box represents the median, with the mean value indicated by ' + '. Where indicated, $p$ values are specified as ‘**' $(p<0.01)$ or '***' $(p<0.001)$ as compared to wild type. 

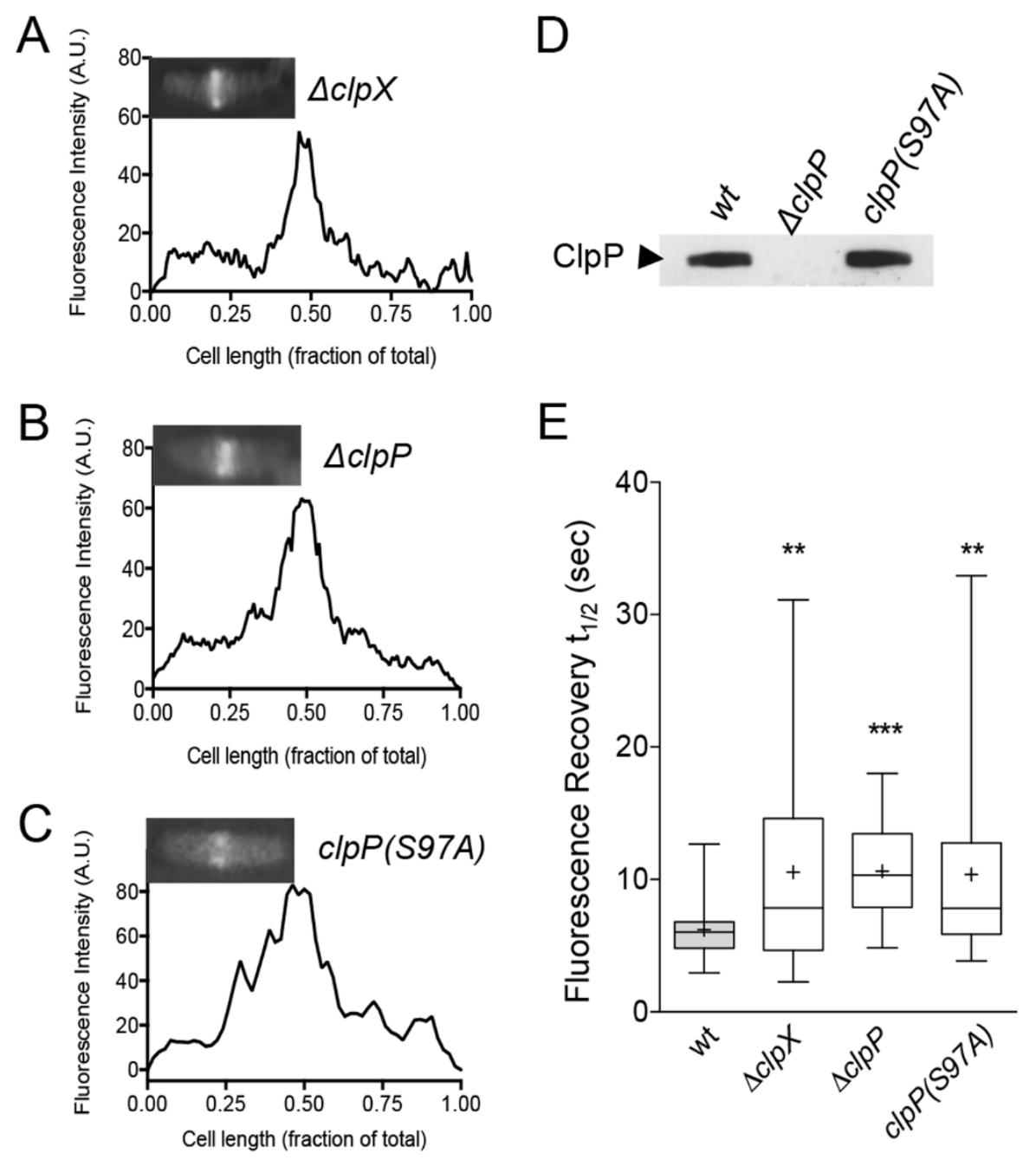
Fig 4. Mutation of ClpX interaction site impairs substrate degradation in vitro and dynamic exchange in vivo. (A) Degradation of $\mathrm{Gfp}_{-} \mathrm{Z}_{\mathrm{C} 67}(3 \mu \mathrm{M})$ and mutant Gfp$\mathrm{Z}_{\mathrm{C} 67}(\mathrm{R} 379 \mathrm{E})(3 \mu \mathrm{M})$ was measured by monitoring loss of fluorescence with time in the presence (black and white circles, respectively) or absence (black and grey lines, respectively) of ClpXP $(1 \mu \mathrm{M})$ where indicated, ATP $(5 \mathrm{mM})$, and a regenerating system. The curves shown are representative of at least three replicates. (B) Fluorescence microscopy of wild type cells (JC0390) expressing Gfp-FtsZ, Gfp-FtsZ(R379E), GfpFtsZ(G105S), Gfp-FtsZ(3527A), or Gfp-FtsZ(G105S, R379E) induced with $140 \mu \mathrm{M}$ arabinose under growth conditions described in Materials and methods. (C) Box and whiskers plot of average recovery half-times of Z-rings in wild type cells (JC0390) expressing Gfp-FtsZ, Gfp-FtsZ(R379E), Gfp-FtsZ(G105S), Gfp-FtsZ(3527A), or GfpFtsZ(G105S, R379E) induced with $140 \mu \mathrm{M}$ arabinose under growth conditions described in Materials and methods. The extent of the box encompasses the interquartile range of the fluorescence recovery half-times, and whiskers extend to the maximum and minimum values. The line within each box represents the median, with the mean value indicated by '+'. Where indicated, $p$ values are specified as '**' $(p<0.01)$, '***' $(p<0.001)$ or 'n.s.' (not significant) as compared to wild type. 
A

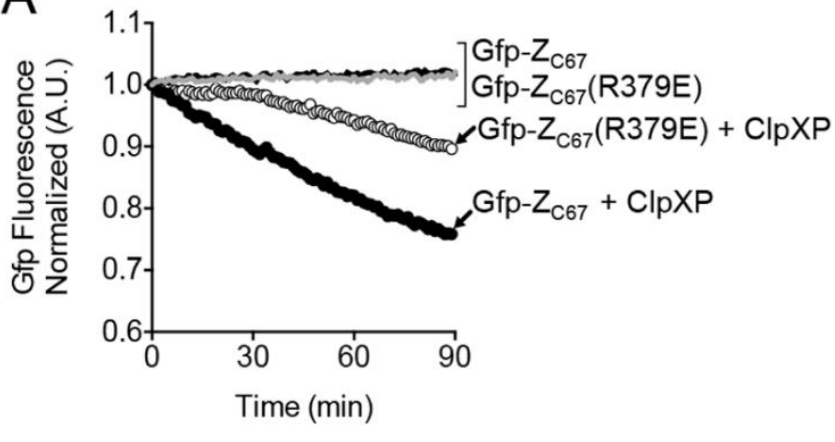

C

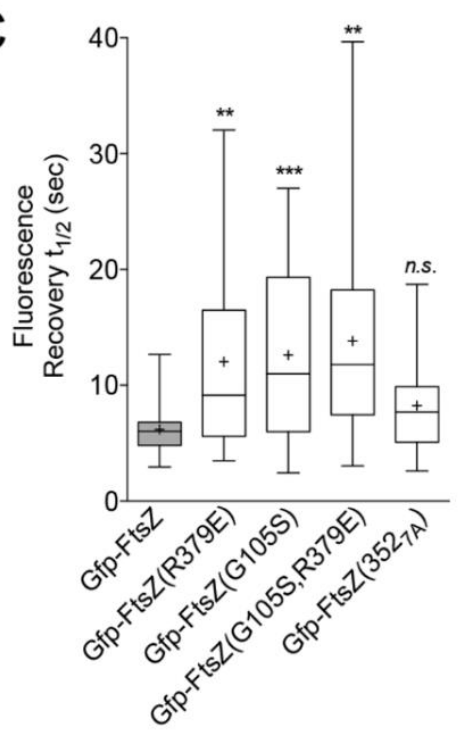

B
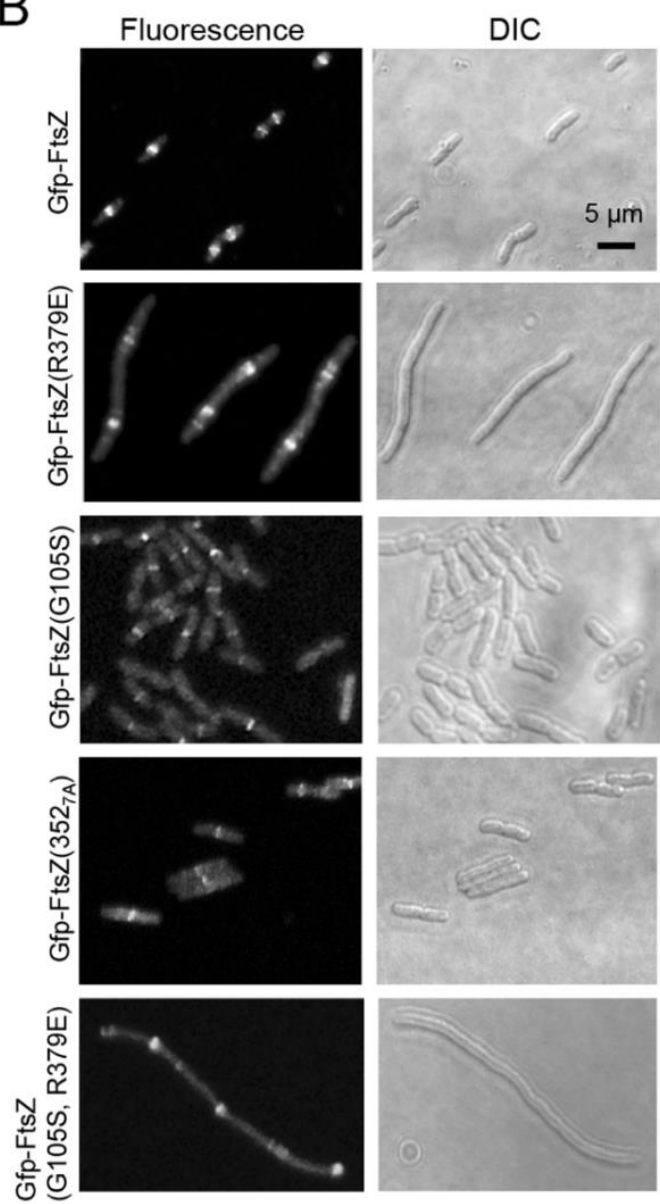
Fig 5. ZipA-Gfp ring assembly and dynamics are unaffected by deletion of $c l p X$. (A) Fluorescence microscopy imaging of ZipA-Gfp rings in cells expressing ZipA-Gfp from the chromosome induced with $10 \mu \mathrm{M}$ IPTG in strains with (MC181) and without $c l p X$ (MV0226) under growth conditions described in Materials and methods. Size bar is 2 $\mu \mathrm{m}$. (B) Box and whiskers plot of average recovery half-times of ZipA-Gfp rings with (MC181) and without $\operatorname{clpX}$ (MV0226). The extent of the box encompasses the interquartile range of the fluorescence recovery half-times, and whiskers extend to the maximum and minimum values. The line within each box represents the median. Where indicated, $p$ values are specified as 'n.s.' (not significant) as compared to wild type. 
A

Fluorescence
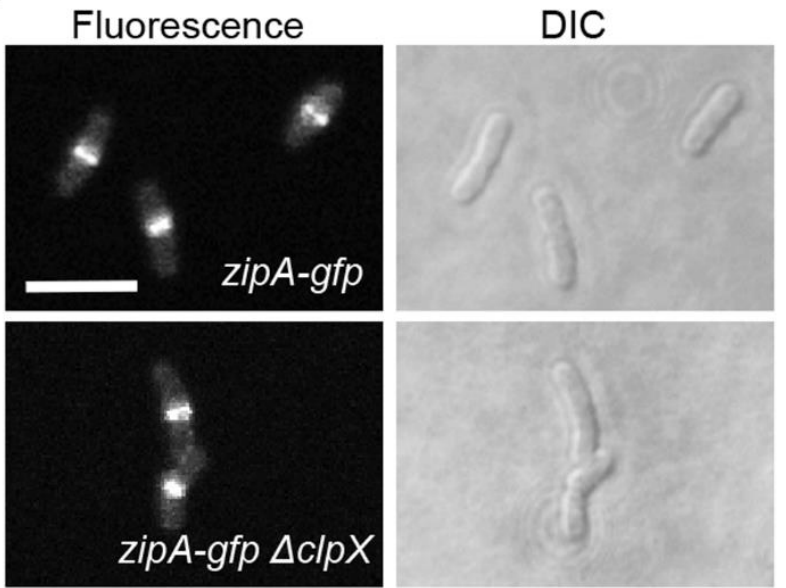

B

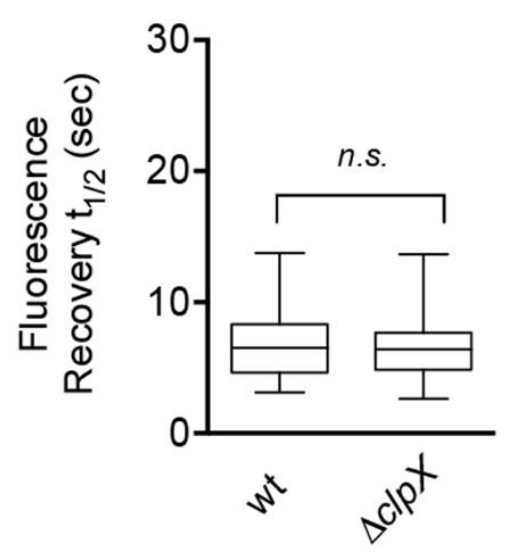


Fig 6. ClpXP degradation of FtsZ polymers. (A) Alexa Fluor labeled FtsZ (24 $\mu \mathrm{M}$ total) was polymerized with GTP $(2 \mathrm{mM})$, where indicated, in the presence of a regenerating system, and then incubated alone, with $\mathrm{ClpXP}(1.6 \mu \mathrm{M})$ and ATP $(5 \mathrm{mM})$ or with ClpX $(1.6 \mu \mathrm{M})$ and ATP $(5 \mathrm{mM})$. Reaction products were crosslinked with DSP, fractionated on a sucrose gradient and quantified by fluorescence. (B) Plot of the rate of degradation with increasing substrate concentration by ClpXP $(1 \mu \mathrm{M})$ and Alexa Fluor labeled FtsZ (0 to $6 \mu \mathrm{M}$ ) with ATP $(5 \mathrm{mM})$, a regenerating system and, where indicated GTP $(2 \mathrm{mM})$. Hill coefficients $\left(\mathrm{n}_{\mathrm{h}}\right)$ were calculated by fitting the data to a nonlinear regression model as described in Materials and methods. 


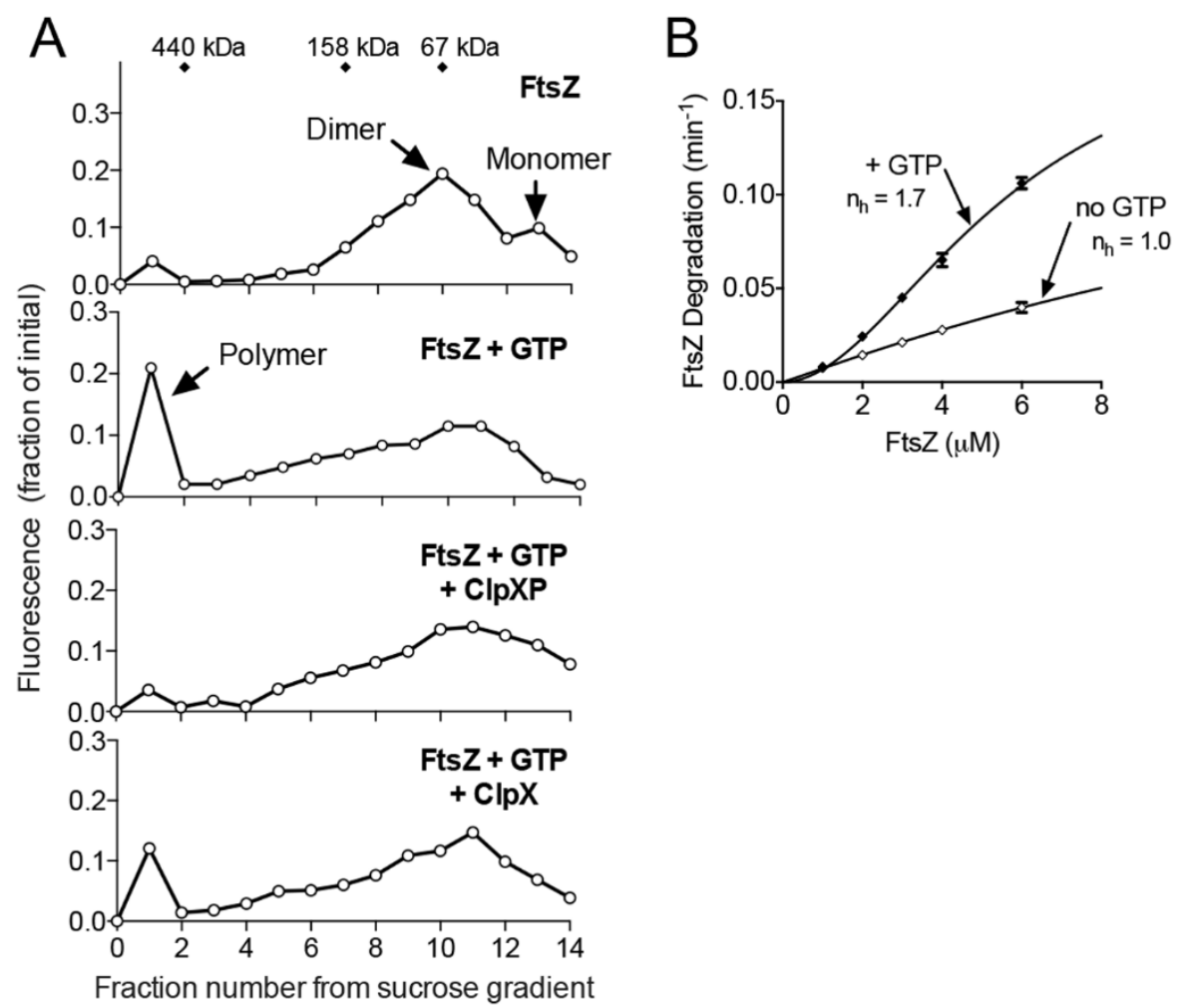


Fig 7. Slow Z-ring fluorescence recovery half-times in strains deleted for other cell division regulators. Fluorescence intensity across the long axis of the cell was measured and plotted for cells deleted for $\min C(\mathrm{JC0395)}(\mathrm{A}), \operatorname{sim} A(\mathrm{MV0198)}(\mathrm{B})$, and zapE (MV0277) (C) as described in Materials and methods. Inset shows the fluorescence image used for quantitation. Individual cells were chosen as representative of the population. (D) Plot for average fluorescence recovery half-times of bleached Z-rings in cells deleted for $\operatorname{minC}$ (JC0395), $\operatorname{sim} A$ (MV0198), and zapE (MV0277). The extent of the box encompasses the interquartile range of the fluorescence recovery half-times, and whiskers extend to the maximum and minimum values. The line within each box represents the median, with the mean value indicated by ' + '. Where indicated, $p$ values are specified as '**' $(p<0.01)$ as compared to wild type. 
A

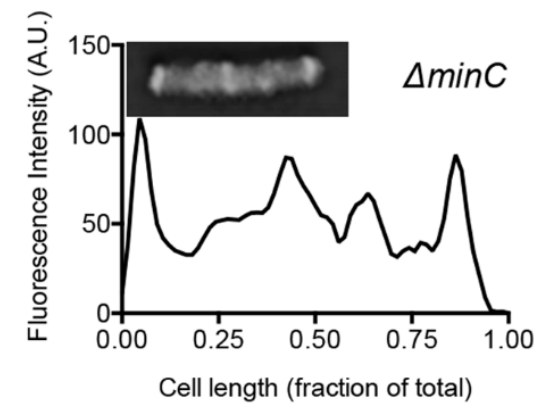

C

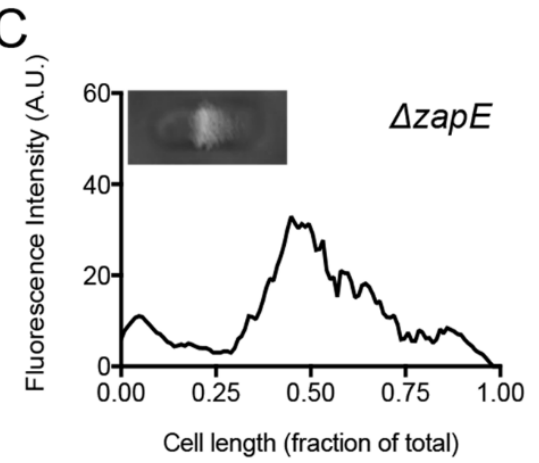

B

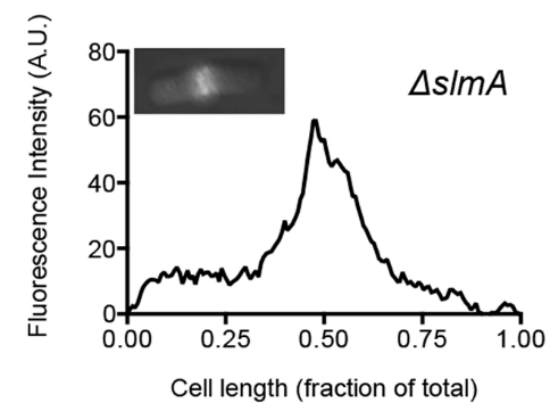

D

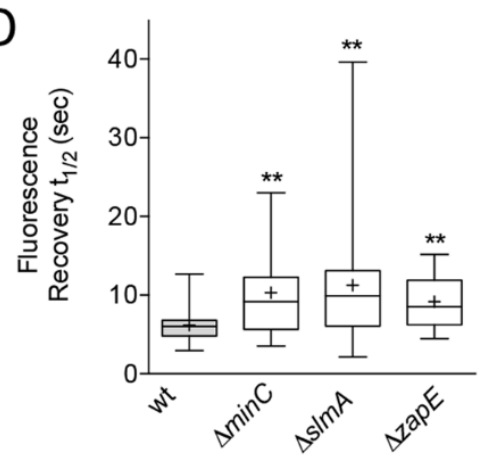




\section{S1 Fig.}

ClpXP degradation of Gfp and ClpX unfolding of FtsZ chimeras in vitro. (A) Degradation of Gfp (3 $\mu \mathrm{M})$ in the presence (black circles) and absence (white circles) of ClpXP $(1 \mu \mathrm{M})$, ATP $(5 \mathrm{mM})$ and a regenerating system was measured by monitoring loss of fluorescence with time. (B) Unfolding of Gfp-Z $Z_{\mathrm{C} 67}(3 \mu \mathrm{M})$ in the presence of ClpX (1 $\mu \mathrm{M})$, ATP $(5 \mathrm{mM})$ and a regenerating system was measured by monitoring loss of fluorescence with time (grey circles). Unfolding of Gfp-FtsZ $(5 \mu \mathrm{M})$ in the presence (green circles) and absence (yellow circles) of GTP, ClpX (1 $\mu \mathrm{M})$, ATP $(5 \mathrm{mM})$ and a regenerating system was measured by monitoring loss of fluorescence with time. For the unfolding of Gfp-FtsZ monomers in the absence of GTP, a regenerating system was used only for ATP containing creatine kinase $(60 \mu \mathrm{g} / \mathrm{ml})$ and phosphocreatine $(5 \mathrm{mg} / \mathrm{ml})$. 


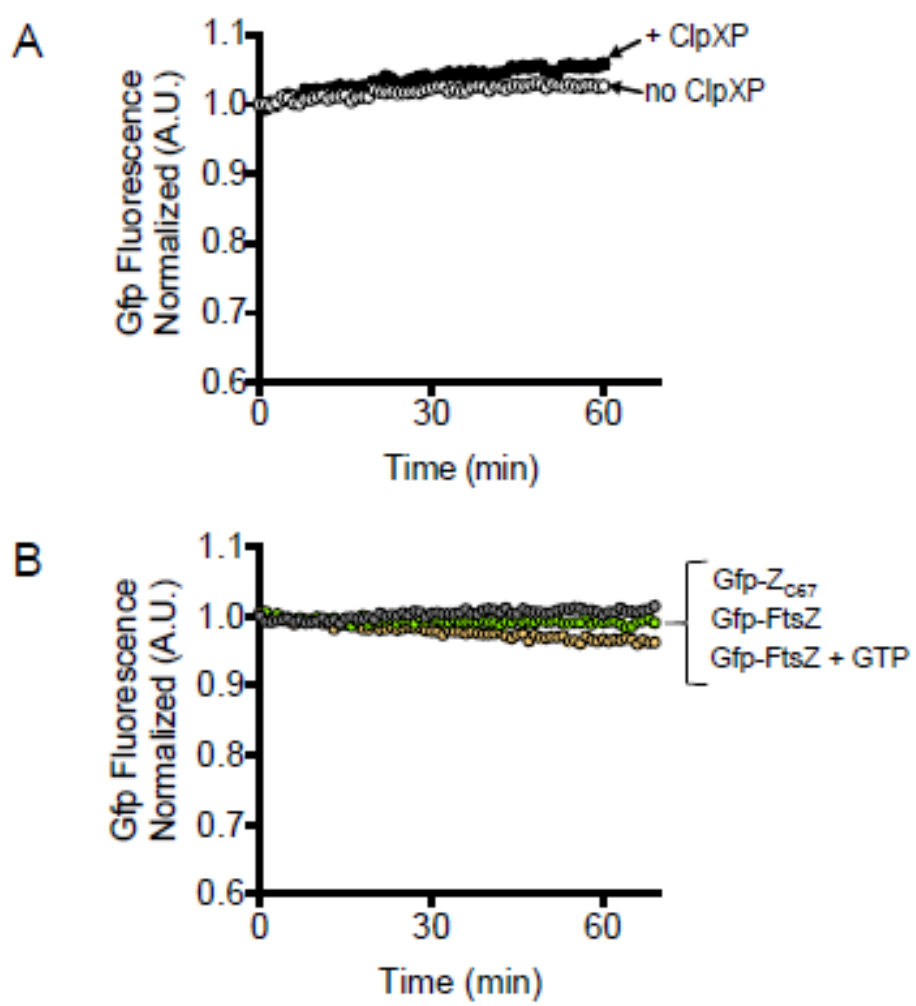




\section{S2 Fig.}

\section{Fluorescence microscopy of Z-rings and replicate recovery curves in clp deficient}

strains. (A) Fluorescence microscopy of wild type cells (JC0390) expressing Gfp-FtsZ induced with $70 \mu \mathrm{M}$ arabinose under growth conditions described in Materials and methods in cells deleted for $\operatorname{clp} X$ (JC0394), clpP (MV0210), with chromosomal clpP(S97A) (MV0256) in place of $c l p P$, cells containing clpP-restored (MV03712) or clpX-restored (MV03722). Size bar is $2 \mu \mathrm{m}$. Replicate recovery curves for Z-rings containing Gfp-FtsZ in cells deleted for $\operatorname{clpX}$ (JC0394) (B), clpP (MV0210) (C), cells expressing chromosomal $\operatorname{clpP}(S 97 A)$ (MV0256) (D) in place of $c l p P$, cells containing clpP-restored (MV03712) (E) or clpX-restored (MV03722) (F). Fluorescence recovery of each replicate was normalized to the maximal fluorescence observed during the recovery period and plotted with time. Immunoblot showing expression of $\mathrm{ClpP}(\mathrm{G})$ or $\mathrm{ClpX}(\mathrm{H})$ is restored in each deletion strain after replacement of the parE-kan cassette by lambda-Red recombination with $c l p P$ or $c l p X$ genes, where indicated. 
A

Fuorescence
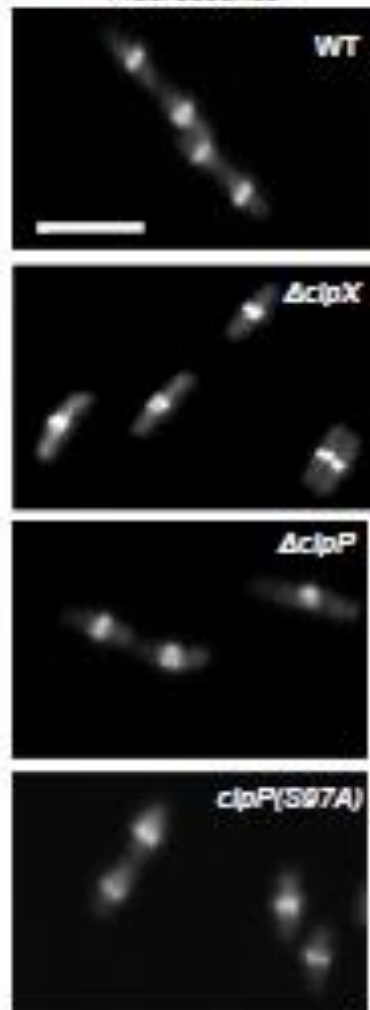

clpp-restored
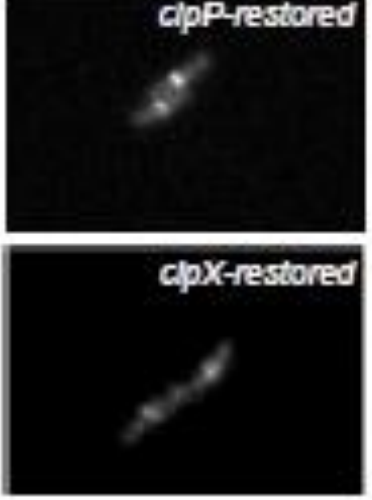

G

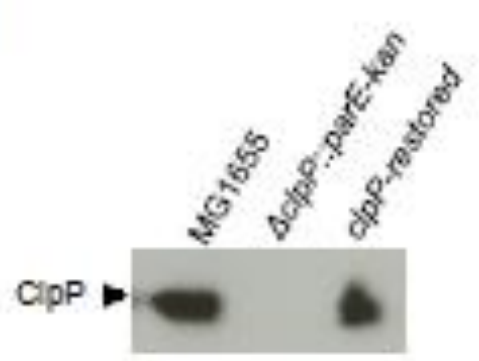

DIC
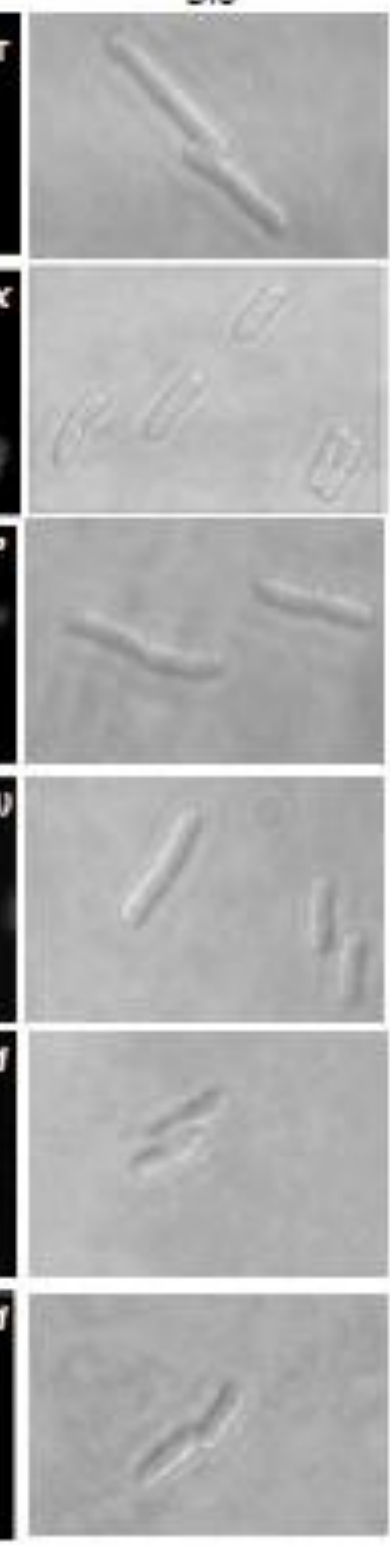

$\mathrm{H}$

D
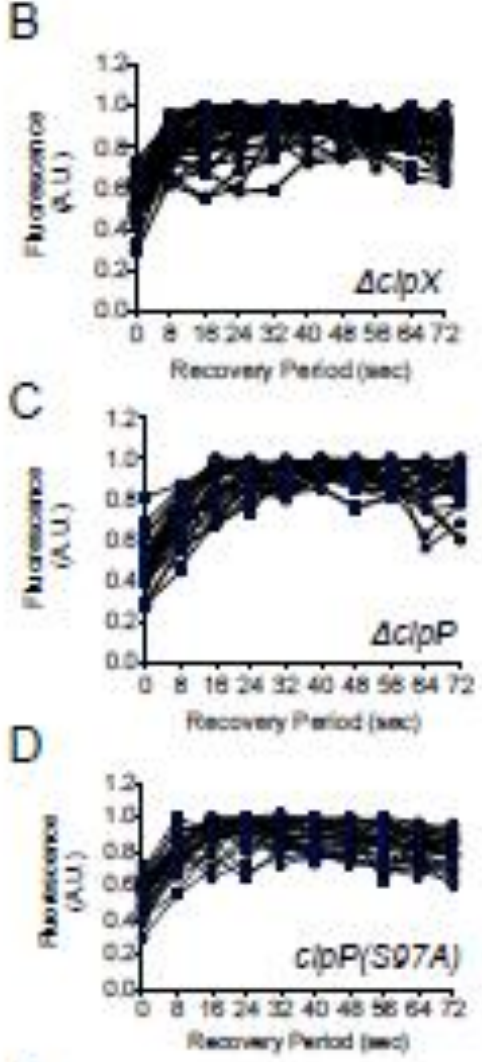

E
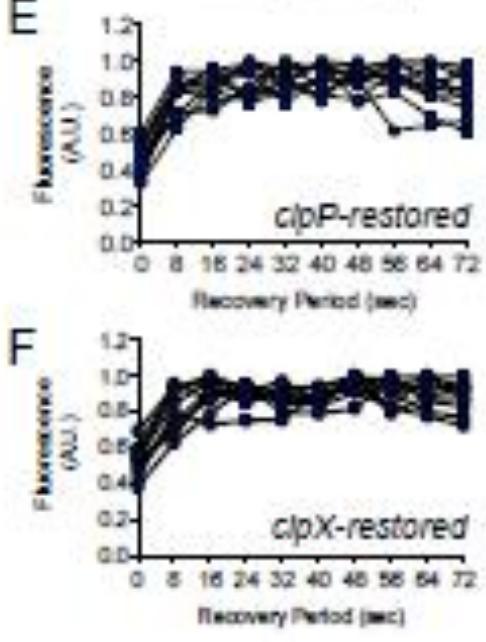


\section{S3 Fig.}

Expression of Gfp-FtsZ at various arabinose concentrations and impact on Z-ring dynamics. (A) Immunoblot for Gfp-FtsZ in wild type cell (JC0390) extracts (1 $\mu \mathrm{g}$ of protein) expressing Gfp-FtsZ induced with 0 , 70, or $140 \mu \mathrm{M}$ arabinose under growth conditions for photobleaching experiments as described in Materials and methods. (B) Plot for average recovery half-times of Z-rings in wild type cells (JC0390) expressing Gfp-FtsZ induced with 70 or $140 \mu \mathrm{M}$ arabinose under growth conditions for photobleaching experiments as described in Materials and methods. 
A

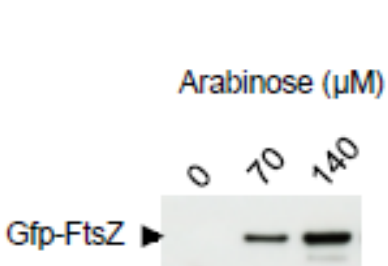

B

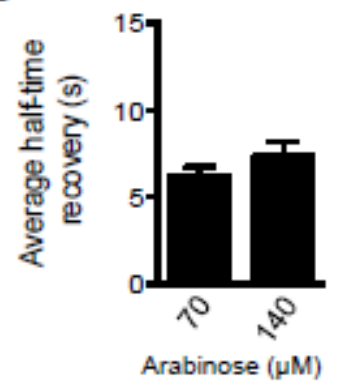




\section{S4 Fig.}

\section{Z-ring localization and fluorescence recovery in cells expressing Gfp-FtsZ(R379E)}

or Gfp-FtsZ(G105S). (A) Fluorescence intensity across the long axis of the cell was measured and plotted for a wild type cell (JC0390) expressing Gfp-FtsZ(R379E). The cell and plot shown are representative of the phenotype caused by expression of GfpFtsZ(R379E). Replicate half-time recovery curves for wild type cells (JC0390) expressing Gfp-FtsZ(R379E) (B), Gfp-FtsZ(G105S) (C), and Gfp-FtsZ(G105S, R379E) (D) induced with $140 \mu \mathrm{M}$ arabinose under growth conditions for photobleaching experiments as described in Materials and methods. Fluorescence recovery of each replicate was normalized to the maximal fluorescence observed during the recovery period and plotted with time. (E) Rates of GTP hydrolysis for wild type FtsZ, FtsZ(R379E), FtsZ(G105S), FtsZ(G105S, R379E), and FtsZ(3527A). (F) Immunoblot for Gfp-FtsZ in wild type (JC0390) cells expressing pBad (empty vector), Gfp-FtsZ, GfpFtsZ(G105S), Gfp-FtsZ(R379E), Gfp-FtsZ(G105S, R379E), or Gfp-FtsZ(3527A) induced with $140 \mu \mathrm{M}$ arabinose under growth conditions described in Materials and methods using antibodies to detect Gfp ( $1 \mu \mathrm{g}$ of protein assayed). 

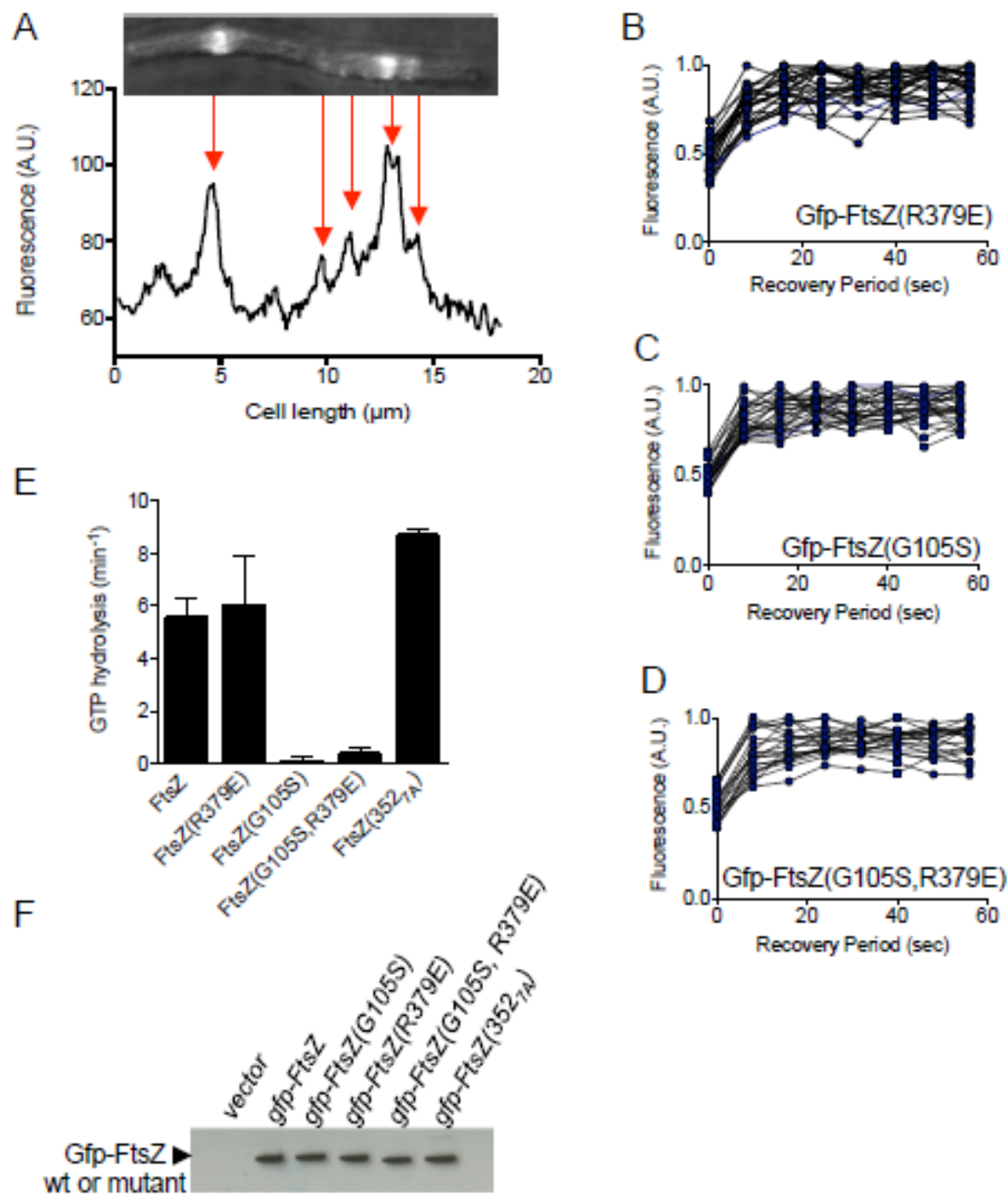


\section{S5 Fig.}

Degradation of FtsZ(3527A) by ClpXP in vitro and fluorescence recovery of GfpFtsZ(352 7 A) in vivo. (A) Degradation reactions containing Alexa Fluor 647 labeled FtsZ $\left(352_{7 \mathrm{~A}}\right)(5 \mu \mathrm{M}$ total $)$ in the presence of $\operatorname{ClpXP}(0.75 \mu \mathrm{M})$, ATP $(5 \mathrm{mM})$, a regenerating system and GTP $(2 \mathrm{mM})$, where indicated, were incubated for 30 minutes and then fluorescent degradation products were collected and quantified. (B) Replicate half-time recovery curves for wild type cells (JC0390) expressing Gfp-FtsZ(3527A) induced with $140 \mu \mathrm{M}$ arabinose under growth conditions for photobleaching experiments as described in Materials and methods. 


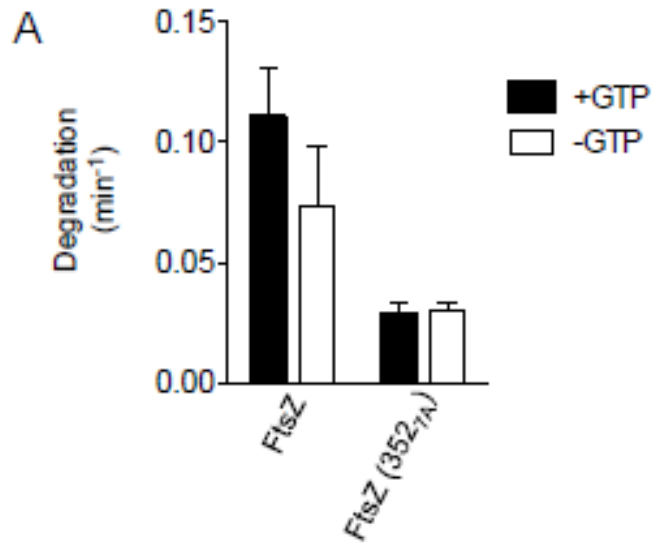

B

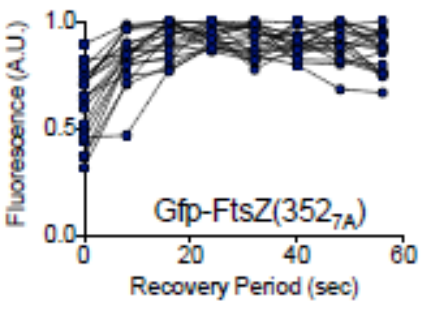




\section{S6 Fig.}

Fluorescence microscopy of Z-rings in cells deleted for other cell division proteins.

(A) Fluorescence microscopy of Z-rings containing Gfp-FtsZ in wild type cells and cells deleted for $\min C$ (JC0395), $\operatorname{sim} A$ (MV0198), zapE (MV0277), and minC-restored (MV03732) under growth conditions for photobleaching experiments as described in Materials and methods. (B) Expression of Gfp-FtsZ in cell lysates (1 $\mu \mathrm{g}$ of protein) induced with $70 \mu \mathrm{M}$ arabinose under growth conditions for photobleaching experiments described in Materials and methods for cells deleted for minC (JC0395), clpX (JC0394), clpP (MV0210), slmA (MV0198), and zapE (MV0277) using antibodies to detect Gfp (C) Replicate fluorescence recovery curves for Z-rings containing Gfp-FtsZ in minC-restored cells (MV03732). (D) Expression of MinC in minC-restored cells by immunoblot using antibodies to MinC. 
A

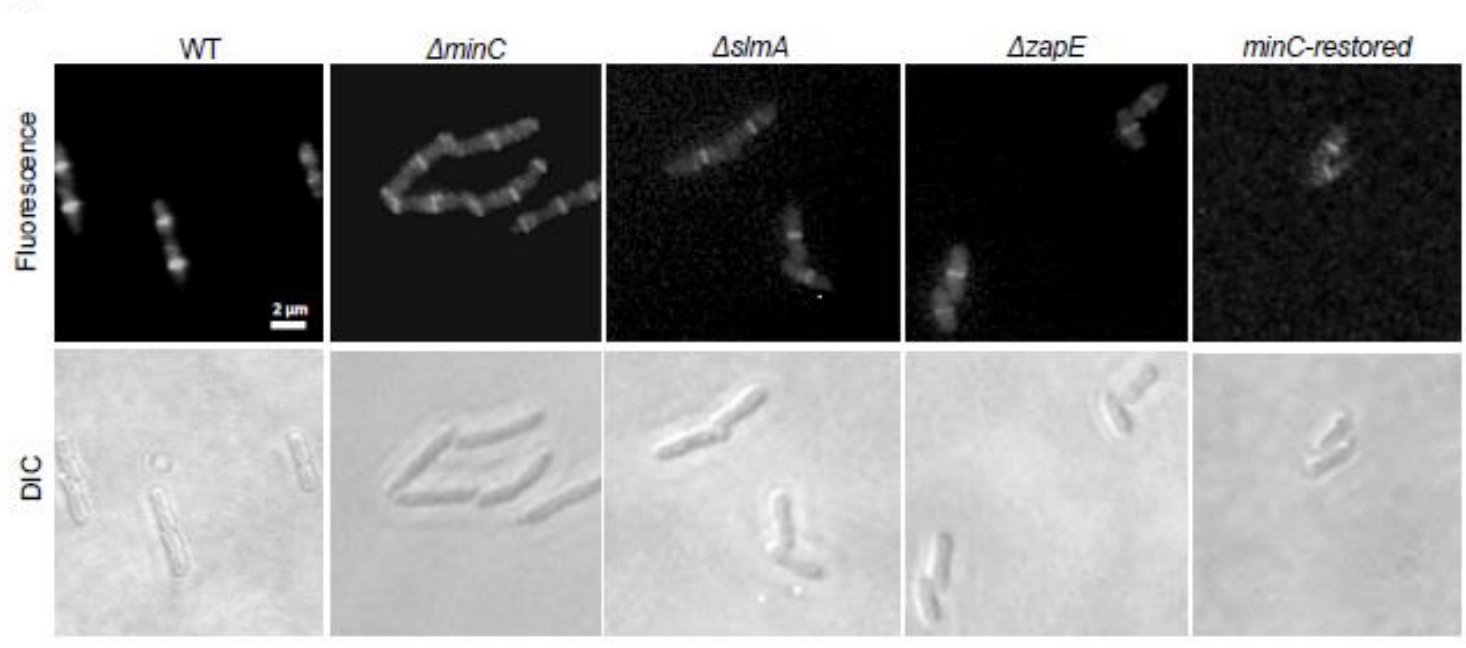

B

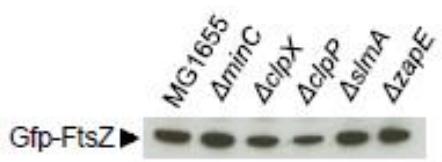

C

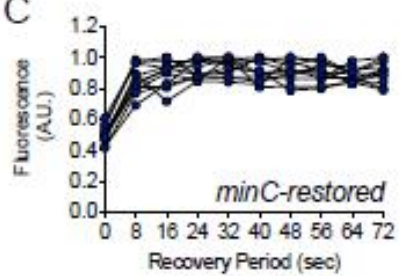

D

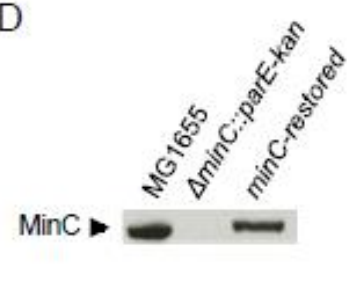




\section{Manuscript V}

Publication status: published in Frontiers in Molecular Biosciences, 2017

Title: The protein chaperone ClpX targets native and non-native aggregated substrates for remodeling, disassembly and degradation with ClpP

Authors: Marissa G. Viola*, Christopher J. LaBreck*, Shannon May*, Joseph Conti, Jodi L. Camberg ${ }^{\#}$

\section{Author Affiliations:}

Department of Cell and Molecular Biology, The University of Rhode Island, Kingston, Rhode Island, United States of America

*These authors contributed equally.

\#Corresponding author: Jodi L. Camberg, 120 Flagg Road, Kingston, RI, 02881; Tel: (401)874-4961; Email: cambergj@uri.edu 


\begin{abstract}
ClpX is a member of the Clp/Hsp100 family of ATP-dependent chaperones and partners with $\mathrm{ClpP}$, a compartmentalized protease, to degrade protein substrates bearing specific recognition signals. ClpX targets specific proteins for degradation directly or with substrate-specific adaptor proteins. Native substrates of ClpXP include proteins that form large oligomeric assemblies, such as MuA, FtsZ and Dps in Escherichia coli. To remodel large oligomeric substrates, ClpX utilizes multivalent targeting strategies and discriminates between assembled and unassembled substrate conformations. Although $\mathrm{ClpX}$ and $\mathrm{ClpP}$ are known to associate with protein aggregates in E. coli, a potential role for ClpXP in disaggregation remains poorly characterized. Here, we discuss strategies utilized by $\mathrm{ClpX}$ to recognize native and non-native protein aggregates and the mechanisms by which $\mathrm{ClpX}$ alone, and with $\mathrm{ClpP}$, remodels the conformations of various aggregates. We show that ClpX promotes the disassembly and reactivation of aggregated Gfp-ssrA through specific substrate remodeling. In the presence of $\mathrm{ClpP}, \mathrm{ClpX}$ promotes disassembly and degradation of aggregated substrates bearing specific ClpX recognition signals, including heat-aggregated Gfp-ssrA, as well as polymeric and heat-aggregated FtsZ, which is a native ClpXP substrate in E. coli. Finally, we show that ClpX is present in insoluble aggregates and prevents the accumulation of thermal FtsZ aggregates in vivo, suggesting that ClpXP participates in the management of aggregates bearing ClpX recognition signals.
\end{abstract}




\section{Introduction}

Maintaining cellular proteostasis relies on chaperone pathways that promote native protein folding. Typical strategies include targeting misfolded, unfolded, and aggregated polypeptides for reactivation or degradation (Bukau and Horwich, 1998;Wickner et al., 1999;Stoecklin and Bukau, 2013). Misfolded proteins are generated during polypeptide elongation and as a complication of environmental stress (Powers and Balch, 2013). The challenges imposed on chaperone systems by proteotoxic stress are especially relevant in pathogenic organisms like E. coli, which experience extreme fluctuations in environmental conditions leading to accumulation of protein aggregates and subsequent proteotoxicity (Mogk et al., 2011). Protein quality control systems reactivate, degrade and remove damaged and aggregated proteins. Under thermal stress in E. coli, the heat shock response provides a cellular defense mechanism and upregulates heat shock protein and chaperone levels to restore proteostasis (Mogk et al., 2011).

In addition to preventing protein aggregation, chaperone proteins mediate aggregate clearance through proteolysis of non-native proteins and aggregation reversal (Hartl et al., 2011;Mogk et al., 2011). Clearance of misfolded proteins in E. coli is carried out by AAA+ (ATPases Associated with diverse cellular Activities) proteins, which initiate substrate recognition, unfolding, and translocation into a proteolytic chamber (ClpP, HslV) (Snider and Houry, 2008;Sauer and Baker, 2011). Several AAA+ proteins, 
such as Lon and FtsH, contain both AAA+ chaperone and proteolytic domains within a single protomer (Sauer and Baker, 2011). The chaperone-protease Lon recognizes exposed aromatic and hydrophobic residues, which may contribute to less stringent substrate selectivity and favor degradation of unfolded or misfolded proteins (Gur and Sauer, 2008).

The Clp ATPases of the AAA+ superfamily can be separated into two functional categories: degradation or disaggregation machines. Degradation machines, including ClpX, ClpA, and HslU form complexes with peptidases ClpP or HslV to remove misfolded proteins or specific substrates (Zolkiewski, 2006). Disaggregation machines, including Hsp104 and its bacterial homolog ClpB, disaggregate and reactivate aggregated proteins by an ATP-dependent mechanism and can function in cooperation with the Hsp70/DnaK system independent of protein degradation (Zolkiewski, 1999;Dougan et al., 2002;Doyle et al., 2007;Sweeny and Shorter, 2016). Through a collaborative mechanism, Hsp70, with Hsp40, binds first to a polypeptide segment of an aggregated protein and then the substrate is remodeled by Hsp104/ClpB (Zietkiewicz et al., 2004;Zietkiewicz et al., 2006;Acebron et al., 2009).

E. coli substrates that are degraded by ClpXP include a variety of cellular proteins, metabolic enzymes and several proteins capable of forming large conformational assemblies, including FtsZ, Dps and MinD (Flynn et al., 2003;Stephani et 
al., 2003;Neher et al., 2006;Camberg et al., 2009;Camberg et al., 2014;Conti et al., 2015). ClpXP can associate with cellular aggregates in E. coli and can promote removal of cellular inclusions, but direct protein disaggregation in vitro is not well characterized for ClpX (Vera et al., 2005;Winkler et al., 2010). An early study suggested that ClpX, in the absence of $\mathrm{ClpP}$, could protect the lambda $\mathrm{O}$ phage protein from aggregation and resolubilize lambda O aggregates (Wawrzynow et al., 1995). In Bacillus subtilis, ClpX also localizes to protein aggregates, suggesting that it may be involved in protein disaggregation (Kruger et al., 2000;Kain et al., 2008;Kirstein et al., 2008)Simmons et al., 2008). ClpX and ClpX substrates are present in polar protein aggregates in E. coli under stress in vivo, suggesting that $\mathrm{ClpX}$ associates with aggregated proteins and participates in their removal (Kain et al., 2008;Maisonneuve et al., 2008;Simmons et al., 2008).

ClpXP comprises an asymmetric, hexameric ring of ClpX docked to two stacked heptameric rings of the ClpP serine protease (Wang et al., 1997;Glynn et al., 2009). Although ClpX has been shown to independently remodel substrates, such as MuA, in the presence of ClpP, hydrophobic 'IGF' loops on the bottom surface of the ClpX hexamer contact hydrophobic pockets on the ClpP tetradecamer, allowing unfolded substrates to access the ClpP proteolytic chamber (Kim et al., 2001;Abdelhakim et al., 2010;Baker and Sauer, 2012). Nucleotide binding by ClpX protomers, in the cleft between the large and small AAA+ subdomains, regulate the position of the subdomains relative to each other; these conformational changes enable $\mathrm{ClpX}$ to couple substrate translocation to ATP 
hydrolysis (Glynn et al., 2009;Baker and Sauer, 2012). Substrates are then translocated into the ClpP chamber for degradation (Baker and Sauer, 2012).

Substrates bind to the ClpX N-domain and to residues in the ClpX central channel (pore-loops) (Bolon et al., 2004;Park et al., 2007;Martin et al., 2008;Baker and Sauer, 2012). The N-domain of $\mathrm{ClpX}$ is separated from the AAA+ domain by a flexible linker and can dimerize independently. The $\mathrm{N}$-domain is important for direct recognition of some substrates, including FtsZ and $\mathrm{MuA}$, as well as adaptor proteins, but is not required for direct recognition of the ssrA-tag (Abdelhakim et al., 2008;Martin et al., 2008; Camberg et al., 2009;Baker and Sauer, 2012). Adaptor proteins, such as RssB or $\mathrm{SspB}$, promote the interaction and engagement of specific substrates, such as RpoS or ssrA-tagged substrates, respectively (Sauer and Baker, 2011). The ssrA tag is an 11residue degron appended to a nascent polypeptide when the ribosome stalls during protein synthesis, targeting the misfolded protein for subsequent degradation (Gottesman et al., 1998; Levchenko et al., 2000).

ClpXP is implicated in the degradation of diverse cellular substrates and more than 100 substrates have been reported (Flynn et al., 2003;Neher et al., 2006). Native substrates of ClpX contain recognition motifs at the N- or C-termini (Flynn et al., 2003). Notably, the essential cell division protein FtsZ in E. coli has two distinct ClpX motifs: one in the flexible linker region and one near the C-terminus (Camberg et al., 2014). FtsZ 
is a tubulin homolog that assembles into linear polymers in vitro and forms the septal ring critical for division in vivo, called the Z-ring (Erickson et al., 2010). ClpXP degrades approximately $15 \%$ of FtsZ proteins during the cell cycle in E. coli and is capable of degrading both monomers and polymers in vitro (Camberg et al., 2009). ClpXP degrades polymers more efficiently, which is consistent with a common strategy of multivalent recognition of substrates by AAA+ ATPases (Davis et al., 2009;Camberg et al., 2014;Ling et al., 2015). In addition to FtsZ, several other ClpXP substrates form large oligomeric structures, including the tetrameric phage protein $\mathrm{MuA}$, the dodecameric bacterial protein Dps, and the bacterial cell division ATPase MinD (Stephani et al., 2003; Neher et al., 2006;Abdelhakim et al., 2010;Conti et al., 2015). Like FtsZ, alternate monomeric and oligomeric conformations of $\mathrm{MuA}$ are also differentially recognized by ClpX (Abdelhakim et al., 2008;Abdelhakim et al., 2010;Ling et al., 2015).

In this study, we use engineered and native substrates to investigate the role of $\mathrm{ClpX}$ and ClpXP in the disassembly and degradation of protein aggregates that bear specific ClpX recognition signals. We observed that ClpX, with and without ClpP, destabilizes Gfp-ssrA aggregates in vitro. The native ClpXP substrate FtsZ forms several discrete conformations, including linear ordered polymers and also heat-induced aggregates. Our results show that ClpXP disassembles both heat-induced and linear polymers containing FtsZ. Finally, we also demonstrate that thermal stress promotes aggregation of FtsZ, which is exacerbated in cells deleted for $\operatorname{clp} X$ or $\operatorname{clp} P$. Together, these results show bona fide chaperone activity for $\mathrm{ClpX}$ in vitro and suggest that $\mathrm{ClpX}$, 
with or without ClpP, may play a broader role in rescue and disassembly of protein aggregates.

\section{Materials and Methods}

\section{Bacterial strains and plasmids}

E. coli strains and plasmids used in this study are described in Table 1. An expression plasmid encoding $\operatorname{FtsZ}(\Delta C 67)$ was constructed by introducing a TAA stop codon (at residue 317 of FtsZ) into pET-FtsZ by site-directed mutagenesis (Camberg et al., 2009).

\section{Expression and purification of proteins}

Gfp-ssrA was purified as previously described (Yakhnin et al., 1998). ClpX, ClpP, FtsZ, and FtsZ( $\Delta$ C67) were each overexpressed in E. coli BL21 ( $\lambda$ DE3) and purified as described (Maurizi et al., 1994;Grimaud et al., 1998;Camberg et al., 2009; Camberg et al., 2014). ClpX(E185Q) was purified as described for wild type ClpX, except the expression strain, E. coli MG1655 $\Delta c l p X$ carrying plasmid pClpX(E185Q), was induced with $1 \%$ arabinose (Table 1) (Camberg et al., 2011). Gfp(uv) containing an $\mathrm{N}$-terminal histidine tag was overexpressed in E. coli BL21 ( $\lambda \mathrm{DE} 3)$ and grown to an $\mathrm{OD}_{600}$ of 1.0 and induced for 3 hours at $30^{\circ} \mathrm{C}$. Cells were lysed by French press in purification lysis buffer (20 mM HEPES, pH 7.5, $5 \mathrm{mM} \mathrm{MgCl}_{2}, 50 \mathrm{mM} \mathrm{KCl}$, and $10 \%$ 
glycerol). Soluble extracts were bound to TALON metal affinity resin (GE Healthcare), eluted with an imidazole gradient, and imidazole was removed by buffer exchange. Protein concentrations are reported as FtsZ monomers, ClpX hexamers, ClpP tetradecamers, and Gfp or Gfp-tagged monomers. For polymerization assays, FtsZ was labeled with Alexa Fluor 488 and active protein (FL-FtsZ) was collected after cycles of polymerization and depolymerization as described (Gonzalez et al., 2003;Camberg et al., 2014).

\section{Dynamic light scattering}

Dynamic light scattering (DLS) measurements were made using a Zetasizer Nano ZS (Malvern Instruments). To determine size distribution, FtsZ ( $5 \mu \mathrm{M})$, aggFtsZ $(5 \mu \mathrm{M})$, Gfp-ssrA $(1.5 \mu \mathrm{M})$ and aggGfp-ssrA $(1.5 \mu \mathrm{M})$ in reaction buffer (50 mM HEPES, $\mathrm{pH}$ 7.5, $100 \mathrm{mM} \mathrm{KCl}$ and $10 \mathrm{mM} \mathrm{MgCl} 2$ ) were added to polystyrene cuvettes and scanned at $23{ }^{\circ} \mathrm{C}$ with a detector angle of $173^{\circ}$ and a $4 \mathrm{~mW}, 633 \mathrm{~nm} \mathrm{He}-\mathrm{Ne}$ laser. The reported intensity-weighted hydrodynamic diameters are based on 15 scans.

\section{Heat denaturation, aggregation, disassembly and reactivation of aggregated substrates}

To heat-inactivate Gfp substrates, Gfp-ssrA $(1.5 \mu \mathrm{M})$ or Gfp(uv) $(1.5 \mu \mathrm{M})$ was added, where indicated, to buffer containing HEPES (50 mM, pH 7.5), $\mathrm{KCl}(100 \mathrm{mM})$, $\mathrm{MgCl}_{2}(10 \mathrm{mM})$, glycerol (10\%) and dithiothreitol (DTT) (2 mM) in a volume of $800 \mu \mathrm{l}$ 
and incubated at $85{ }^{\circ} \mathrm{C}$ for 15 minutes. Immediately following heat-treatment, the denatured substrate was placed on ice for 2 minutes and added to a reaction $(50 \mu \mathrm{l})$

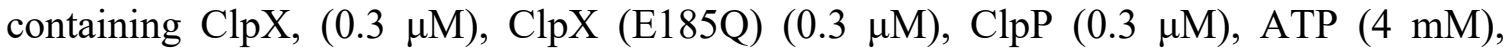
ATP $\gamma \mathrm{S}(1 \mathrm{mM})$, or ADP $(2 \mathrm{mM})$, where indicated. Samples containing ATP were supplemented with an ATP-regenerating system containing phosphocreatine $\left(5 \mathrm{mg} \mathrm{ml}^{-1}\right)$ and creatine kinase $(\mathrm{CK})\left(60 \mu \mathrm{g} \mathrm{ml}^{-1}\right)$. Fluorescence recovery was monitored by measuring fluorescence in a Cary Eclipse fluorometer with excitation and emission wavelengths set at $395 \mathrm{~nm}$ and $510 \mathrm{~nm}$, respectively. Readings were corrected for background signal by subtracting the fluorescence of buffer. Rates were calculated by fitting to a one-phase association model in GraphPad Prism (version 6.0b). Disaggregation was monitored by $90^{\circ}$-angle light scatter with excitation and emission wavelengths set to $550 \mathrm{~nm}$. Readings were corrected for background signal by subtracting the scatter of the buffer and then plotted as percent of the initial turbidity. Heat-induced aggregation of Gfp-ssrA with time was monitored by $90^{\circ}$-angle light scatter with the temperature of the cuvette holder set to $80{ }^{\circ} \mathrm{C}$ using a circulating water bath.

To inactivate native FtsZ substrates, FtsZ and FtsZ( $\Delta \mathrm{C} 67)(5 \mu \mathrm{M})$ were heated for 15 minutes in reaction buffer (20 mM HEPES, pH 7.5, $100 \mathrm{mM} \mathrm{KCl,} 10 \mathrm{mM} \mathrm{MgCl}$ ) in a volume of $120 \mu \mathrm{l}$ at $65^{\circ} \mathrm{C}$, then cooled on ice for 40 seconds, and held at $23{ }^{\circ} \mathrm{C}$ until addition to reactions (60 $\mu \mathrm{l}$ volume) containing $\mathrm{ClpX}(0.5 \mu \mathrm{M}$ or $1 \mu \mathrm{M}), \mathrm{ClpX}(\mathrm{E} 185 \mathrm{Q})$ $(0.5 \mu \mathrm{M}), \mathrm{ClpP}(1 \mu \mathrm{M})$, ATP $(4 \mathrm{mM})$ and an ATP-regenerating system (phosphocreatine at $5 \mathrm{mg} \mathrm{ml}^{-1}$ and creatine kinase at $60 \mu \mathrm{g} \mathrm{ml}^{-1}$ ), where indicated. Disaggregation was monitored by $90^{\circ}$-angle light scatter with excitation and emission wavelengths set to 450 
nm. Readings were corrected for background signal by subtracting the scatter of the buffer and then plotted as percent of the initial turbidity. Heat-induced aggregation of FtsZ with time was monitored by $90^{\circ}$-angle light scatter with the temperature of the cuvette holder set to $65^{\circ} \mathrm{C}$ using a circulating water bath.

\section{Polymerization and GTP hydrolysis assays}

FL-FtsZ was incubated with the GTP analog GMPCPP $(0.5 \mathrm{mM})$ in the presence of increasing concentrations of $\mathrm{ClpX}$ and $\mathrm{ClpP}(0,0.25,0.5$ or $1 \mu \mathrm{M})$ as indicated and in the presence of phosphocreatine at $5 \mathrm{mg} \mathrm{ml}^{-1}$ and creatine kinase at $60 \mu \mathrm{g} \mathrm{ml}^{-1}$. Samples were incubated for 3 minutes in buffer containing MES (50 mM, pH 6.5), $\mathrm{KCl}(100 \mathrm{mM})$ and $\mathrm{MgCl}_{2}(10 \mathrm{mM})$ at $23{ }^{\circ} \mathrm{C}$, then centrifuged at $129,000 \mathrm{x} g$ in a Beckman TLA 120.1 rotor for 30 minutes. Pellets were resuspended in $0.2 \mathrm{M} \mathrm{NaCl}$ with $0.01 \%$ Triton $\mathrm{X}-100$ $(100 \mu \mathrm{l})$ and the fluorescence associated with FL-FtsZ for supernatants and pellets was measured using a Cary Eclipse spectrophotometer. GTP hydrolysis rates for FtsZ and $\operatorname{FtsZ}(\Delta C 67)$ were measured before and after aggregation using the Biomol Green (Enzo Life Sciences) detection reagent as described (Camberg et al., 2014).

\section{Heat shock of wild type and deletion strains}

E. coli wild type and deletion strains were grown overnight, diluted 1:100 in fresh Lennox broth the next day and grown at $30{ }^{\circ} \mathrm{C}$ to an $\mathrm{OD}$ of 0.4 . All strains were incubated in a water bath at $50{ }^{\circ} \mathrm{C}$ for 1 hour, followed by recovery at $30{ }^{\circ} \mathrm{C}$ for 35 
minutes. Cells were harvested by centrifugation and lysed with Bacterial Protein Extraction Reagent (B-PER) (ThermoFisher Scientific) (2 ml) and lysozyme (25 $\left.\mu \mathrm{g} \mathrm{ml}^{-1}\right)$. Insoluble fractions were collected by centrifugation at $15,000 \mathrm{x} g$ for 5 minutes at $4{ }^{\circ} \mathrm{C}$, resuspended in lithium dodecyl sulfate sample buffer and analyzed by reducing SDSPAGE. Total proteins were transferred to a nitrocellulose membrane and visualized by Ponceau (Fisher Scientific) staining and membranes were immunoblotted using antibodies to ClpX and FtsZ (Camberg et al., 2009;2011). Band intensities were analyzed by densitometry (NIH ImageJ), normalized to the intensity of the average of the 'no heat' sample, and evaluated for significance by the Mann-Whitney test. Where indicated, to test a mild heat shock condition, cells were incubated in a water bath at $42{ }^{\circ} \mathrm{C}$ for 30 minutes, followed by recovery at $30{ }^{\circ} \mathrm{C}$ for 35 minutes, and analyzed as described. 


\section{Results}

\section{ClpXP degrades aggregates in vitro}

To determine if ClpX can remodel protein substrates from the aggregated state, we used the fusion protein, Gfp-ssrA, which forms aggregates upon heat treatment (Zietkiewicz et al., 2004;Zietkiewicz et al., 2006). Gfp-ssrA is rapidly degraded by ClpXP and has been extensively studied to understand substrate targeting by ClpXP. The Gfp moiety is widely used in protein disaggregation assays because it forms nonfluorescent aggregates when heated, but is disaggregated and reactivated by several chaperone systems (Zietkiewicz et al., 2004;Zietkiewicz et al., 2006). Therefore, we heated Gfp-ssrA at $85{ }^{\circ} \mathrm{C}$ for 15 minutes to induce aggregation (aggGfp-ssrA), resulting in an $86 \%$ loss of fluorescence emitted (Figure 1A). Next, to measure the distribution of aggregates by size after heating, we performed dynamic light scattering (DLS) of untreated and heat-denatured Gfp-ssrA. We observed that without heating, the particle sizes are uniform with an average hydrodynamic diameter of 8-10 nm (Figure 1B). After heating, aggregates are approximately $500-600 \mathrm{~nm}$, and there is a narrow distribution of particle sizes and no small particles (i.e., less than $100 \mathrm{~nm}$ ) (Figure 1C). Upon heat-treatment, aggregation of Gfp-ssrA $(1.5 \mu \mathrm{M})$ occurs rapidly and plateaus by 10 minutes by $90^{\circ}$-angle light scattering (Figure 1D). The heat inactivation is irreversible since incubation of aggregated Gfp-ssrA (aggGfp-ssrA) alone does not lead to appreciable fluorescence reactivation, which is consistent with

previous reports using Gfp (Figure S1) (Zietkiewicz et al., 2004). To determine if 200 
ClpXP can bind to aggregates and degrade them, we incubated aggGfp-ssrA with ClpXP and monitored turbidity by $90^{\circ}$-angle light scattering. Incubation of aggGfpssrA with ClpXP led to a 35\% loss of turbidity in 2 hours (Figure 1E). However, when ClpXP was omitted from the reaction, there was very little change in turbidity over time (5\% loss in 2 hours) (Figure 1E). This suggests that ClpXP targets aggregated substrates for degradation. To determine if degradation is required to reduce turbidity, we omitted $\mathrm{ClpP}$ and observed that $\mathrm{ClpX}$ is capable of reducing sample turbidity by $15 \%$ in 2 hours (Figure 1E). Finally, when ATP was omitted from the reaction containing ClpXP, we observed a less than $10 \%$ reduction in the turbidity of the reaction (Figure 1E). To confirm that ClpXP degrades aggGfp-ssrA, we incubated aggGfp-ssrA with combinations of $\mathrm{ClpX}, \mathrm{ClpP}$ and $\mathrm{ATP}$, and sampled degradation reactions after 2 hours. We observed that in the presence of ClpXP, aggGfp-ssrA is degraded, but not when ClpP or ATP was omitted (Figure 1F). Together, these results demonstrate that ClpXP targets aggregates for ATP-dependent degradation and that ClpX is also capable of promoting disassembly in the absence of ClpP.

FtsZ is a well-characterized ClpXP substrate that is essential for cell division and forms linear polymers in vitro in the presence of GTP (Erickson et al., 2010). We previously showed that ClpXP binds to GTP-stimulated FtsZ polymers and promotes FtsZ degradation (Camberg et al., 2009). ClpXP also recognizes and degrades nonpolymerized FtsZ, but less efficiently than polymerized FtsZ (Camberg et al., 2009). In vitro, FtsZ rapidly aggregates when heated at $65{ }^{\circ} \mathrm{C}$ and this aggregation is 
associated with an increase in overall light scatter and a 97\% loss of GTPase activity (Figure 2A and 2B). FtsZ, which purifies as a mixture of monomers (40.4 kDa) and dimers $(80.8 \mathrm{kDa})$, has an average hydrodynamic diameter of $10-15 \mathrm{~nm}$ by DLS (Figure 2C). Heat treatment of FtsZ $(5 \mu \mathrm{M})$ at $65^{\circ} \mathrm{C}$ produces several particle sizes, including small $(30-40 \mathrm{~nm})$ and large aggregates (>300 nm) (Figure 2D). To determine if ClpXP reduces the turbidity associated with aggregated FtsZ (aggFtsZ), we incubated aggFtsZ with ClpXP and ATP and observed a 40\% loss of turbidity after incubation with ClpXP for 2 hours (Figure 2E). However, in the absence of ClpXP, the light scatter signal remained stable for aggFtsZ (Figure 2E). Incubation of ClpX with aggFtsZ also resulted in a $25 \%$ loss in light scatter, suggesting that ClpX also promotes disassembly of aggregates similar to what we observed for aggGfp-ssrA (Figure 2E and 1E).

Next, to confirm that aggFtsZ is degraded by ClpXP, we assembled reactions containing combinations of aggFtsZ, ClpX, ClpP and ATP and sampled these reactions at 0 and 120 minutes for analysis by SDS-PAGE. We observed that in the presence of ClpXP and ATP, 50\% of the total aggFtsZ in the reaction is lost to degradation after 120 minutes (Figure 2F). Omission of either ClpP or ATP from the reaction prevents loss of aggFtsZ (Figure $2 \mathrm{~F}$ ). These results indicate that ClpXP degrades aggFtsZ. Furthermore, the amount of aggFtsZ after incubation with ClpX is unchanged despite the decrease in light scatter detected, suggesting that ClpX can disaggregate aggFtsZ (Figure 2E and 2F). 
In addition to forming aggregates upon heating, FtsZ also assembles into a linear head-to-tail polymer, which is a native, ordered aggregate, and distinct from the disordered aggregates which are induced by heating (aggFtsZ). We compared the loss of aggFtsZ by ClpXP to a similar reaction monitoring loss of native polymerized FtsZ, which is a known substrate of ClpXP. Like aggFtsZ, we also observed a 50\% loss of polymeric FtsZ, stabilized by the GTP analog GMPCPP, after 120 minutes in reactions containing ClpXP and ATP (Figure 2F). GMPCPP promotes the assembly of stable polymers that are far less dynamic than polymers assembled with GTP (Lu et al., 2000). To test if ClpXP disassembles GMPCPP-stabilized FtsZ polymers, we incubated pre-assembled polymers with ClpXP and ATP. Then, we collected polymers by high-speed centrifugation. In these assays, we used active fluorescent FtsZ, labeled with Alexa fluor 488 (FL-FtsZ), to quantify the amount of polymerized FtsZ in the pellet fraction and soluble FtsZ in the supernatant. We observed that after incubation of GMPCPP-stabilized FtsZ polymers with increasing concentrations of ClpXP ( $0 \mu \mathrm{M}$ to $1 \mu \mathrm{M}$ ), few FtsZ polymers were recovered in the pellet fractions containing ClpXP (26\% of the total FtsZ was recovered in the reaction containing $1 \mu \mathrm{M} \mathrm{ClpXP}$ ), indicating that ClpXP is highly effective at promoting the disassembly of GMPCPPstabilized FtsZ polymers (Figure 2G).

\section{ClpX reactivates heat-aggregated Gfp-ssrA}


Incubation of ClpX with aggGfp-ssrA resulted in loss of turbidity, suggesting that $\mathrm{ClpX}$ may function independently of $\mathrm{ClpP}$ to reactivate substrates (Figure 1E). Reactivation of misfolded proteins may occur through binding and stabilization of intermediates enabling proteins to adopt the native folded conformation, or through ATP-dependent chaperone-assisted unfolding. To determine if ClpX, which recognizes the ssrA amino acid sequence, is able to reactivate aggGfp-ssrA, we monitored fluorescence of aggGfp-ssrA in the presence and absence of ClpX and ATP. AggGfp-ssrA regains very little fluorescence alone, approximately 20 units, which is $8 \%$ of the initial fluorescence lost upon heating; however, in the presence of ClpX, fluorescence recovers rapidly in the first 10 minutes of the reaction and then plateaus, regaining approximately 85 units, which is $27 \%$ of the initial fluorescence lost upon heating (Figure 3A).

ClpX catalyzes ATP-dependent unfolding of substrates (Kim et al., 2000;Singh et al., 2000). To determine if ATP is essential for reactivation, we incubated aggGfpssrA with ClpX under various nucleotide conditions including with ATP, the ATP analog ATPyS, ADP and omission of nucleotide. We observed an $82 \%$ slower rate of fluorescence reactivation when $\mathrm{ClpX}$ and aggGfp-ssrA were incubated with ATPyS than with ATP $\left(0.02 \mathrm{AU} \mathrm{min}{ }^{-1}\right.$ and $0.11 \mathrm{AU} \mathrm{min}^{-1}$, respectively), and no recovery over background with ADP or without nucleotide (Figure 3B). Reactivation by ClpX and ATP is prevented in the presence of ClpP, and the residual fluorescence after heat treatment is lost upon degradation (Figure S2). Together, these results indicate that 
ClpX requires ATP to reactivate Gfp-ssrA and, surprisingly, that ATPyS is also capable of promoting reactivation, although at a much slower rate than ATP (Figure 3B).

\section{Reactivation and disaggregation by $\mathrm{ClpX}$ requires a specific recognition sequence}

Next, we determined if a ClpX recognition motif is important for efficient recognition of aggregated substrates by $\mathrm{ClpX}$. We compared reactivation of aggGfpssrA with heat-aggregated Gfp (aggGfp) without an ssrA tag. We observed that after incubation with $\mathrm{ClpX}$ and ATP for 60 minutes, approximately 30 units of fluorescence were recovered, which is $8 \%$ of the initial pre-heat fluorescence, indicating that aggGfp is a poor substrate for reactivation by ClpX (Figure 4A). In contrast, aggGfpssrA recovered 33\% (>100 units) of the initial pre-heat fluorescence after incubation with ClpX (Figure 4A).

Two regions of FtsZ are important for promoting degradation of E. coli FtsZ by ClpXP, one in the unstructured linker region (amino acids 352-358) and one near the C-terminus (residues 379 through 383) (Camberg et al., 2014). Using a truncated FtsZ mutant protein, FtsZ( $\Delta$ C67), which is deleted for 67 C-terminal amino acid residues, including both regions involved in ClpX recognition, we tested if $\mathrm{ClpXP}$ reduces the light scatter in reactions containing heat-aggregated $\operatorname{FtsZ}(\Delta C 67)$ 
[aggFtsZ( $\Delta \mathrm{C} 67)]$. We heated $\mathrm{FtsZ}(\Delta \mathrm{C} 67)$ at $65^{\circ} \mathrm{C}$ for 15 minutes, the condition that promotes aggregation of full length FtsZ, and confirmed that heat treatment resulted in an $84 \%$ loss of GTP hydrolysis activity and an increase in light scatter, which is stable over time (Figure 4B and 4C). In the presence of ClpXP, we observed no decrease in light scatter for aggFtsZ( $\triangle \mathrm{C} 67)$ after incubation for 120 minutes (Figure 4C), which is expected since $\mathrm{FtsZ}(\Delta \mathrm{C} 67)$ is a poor substrate for ClpXP degradation (Figure S3). Together, these results demonstrate that for $\mathrm{ClpX}$ to recognize aggregates and promote disaggregation, disassembly and/or reactivation, a ClpX recognition motif is required.

\section{Impaired reactivation by $\mathrm{ClpX}(\mathrm{E} 185 \mathrm{Q})$}

ATP is required for reactivation of aggGfp-ssrA, however, it is unknown if this event requires ATP-hydrolysis and substrate unfolding. Therefore, we used the ClpX mutant protein $\mathrm{ClpX}(\mathrm{E} 185 \mathrm{Q})$, which has a mutation in the Walker B motif and is defective for ATP-hydrolysis, but interacts with substrates (Hersch et al., 2005;Camberg et al., 2014). We observed that ClpX(E185Q) is defective for disaggregation of aggGfp-ssrA by monitoring turbidity by $90^{\circ}$-angle light scatter of reactions containing aggGfp-ssrA, ClpX(E185Q) and ATP (Figure 5A). We also tested if aggFtsZ is disassembled by $\mathrm{ClpX}(\mathrm{E} 185 \mathrm{Q})$, and observed no reduction in light scatter in reactions containing aggFtsZ, $\mathrm{ClpX}(\mathrm{E} 185 \mathrm{Q})$ and $\mathrm{ATP}$ after 120 minutes compared to ClpX (Figure 5B). Finally, we tested if reactivation of aggGfp-ssrA requires ATP hydrolysis using $\mathrm{ClpX}(\mathrm{E} 185 \mathrm{Q})$ instead of $\mathrm{ClpX}$. We observed that $\mathrm{ClpX}(\mathrm{E} 185 \mathrm{Q})$ promotes a small amount of reactivation of aggGfp-ssrA and restores 
fluorescence, but to a much lesser extent than the level observed for wild type ClpX (Figure 5C). These results suggest that ATP hydrolysis by $\mathrm{ClpX}$ is required to promote efficient reactivation of aggGfp-ssrA and disassembly of large complexes containing aggFtsZ or aggGfp-ssrA (Figure 5A, 5B and 5C).

\section{ClpXP prevents accumulation of FtsZ aggregates in vivo under extreme thermal} stress

$\mathrm{ClpX}$ and $\mathrm{ClpP}$ were previously reported to localize to protein aggregates in $E$. coli, suggesting that ClpXP may target aggregates in vivo for direct degradation (Winkler et al., 2010). We used the native ClpXP substrate FtsZ, which aggregates upon heat treatment, to determine if $\mathrm{ClpX}$ and/or ClpXP modulates FtsZ aggregate accumulation after thermal stress by comparing the levels of FtsZ present in insoluble cell fractions (Figure 2A and 6A). Wild type cells and cells deleted for $c l p X, c l p P$, $c l p B, c l p A, d n a K, l o n, h s l U$ and $h s l V$ were exposed to heat shock and insoluble protein fractions were collected and analyzed by immunoblot. We observed that FtsZ was present in the insoluble fraction of wild type cells (BW25113), and this amount was $42 \%$ higher in cells exposed to heat shock at $50{ }^{\circ} \mathrm{C}$ (Figure 6A and S4A). However, FtsZ levels were even higher in the insoluble fractions of $\triangle c l p X$ and $\triangle c l p P$ strains compared to the parental strain (2.4-fold and 2.3-fold, respectively), although the amount of total protein was similar to the wild type strain exposed to heat shock (Figure S4B). We detected less protein overall in the $\Delta d n a K$ strain after recovery, but this strain also had poor viability after heat shock and recovery (Figure S4C). In 
addition, we also detected $\mathrm{ClpX}$ in the insoluble fraction in all strains except the $\operatorname{clpX}$ deletion strain (Figure S4A). Next, we conducted a mild heat shock, $42{ }^{\circ} \mathrm{C}$ for 30 minutes, followed by recovery, and observed that deletion of $c l p B$ had a larger effect on the accumulation of insoluble FtsZ than deletion of $\operatorname{clpX}$ (Figure S4D). To determine the relative contributions of either $\operatorname{clp} B$ or $\operatorname{clp} X$ during a 40 minute recovery period after incubation at $50{ }^{\circ} \mathrm{C}$, we analyzed insoluble FtsZ levels at 20 minute time intervals during recovery (Fig. 6B). Notably, we observed that in cells deleted for clpX, insoluble FtsZ was present immediately after heat treatment and continued to accumulate throughout the recovery period to a greater extent than in wild type or $c l p B$ deletion cells. These results suggest that ClpXP prevents accumulation of FtsZ aggregates in cells exposed to extreme thermal stress. Since we observed that insoluble FtsZ levels were elevated in $\Delta c l p B$ strains exposed to mild heat shock (Figure S4D), we repeated the recovery time course in $\operatorname{clp} X$ and $\operatorname{clpB}$ deletion strains after mild heat shock, $42{ }^{\circ} \mathrm{C}$ for 30 minutes, to monitor insoluble FtsZ levels (Figure S4E). We observed that insoluble FtsZ accumulates during the recovery period in $c l p B$ deletion strains after mild heat shock (Figure S4E).

Finally, if ClpXP is active in cells after severe heat shock, then it should not be a thermolabile protein. To determine if $\mathrm{ClpXP}$ remains active after exposure to $50{ }^{\circ} \mathrm{C}$ in vitro, we incubated $\mathrm{ClpXP}$ in buffer at $50{ }^{\circ} \mathrm{C}$ for one hour, and then measured activity after addition of Gfp-ssrA by monitoring the loss of Gfp-ssrA fluorescence. We observed that ClpXP remained active for unfolding and degradation of Gfp-ssrA after incubation at $50{ }^{\circ} \mathrm{C}$ for one hour (Figure S4F). As a control, ClpXP was also 
incubated in buffer at $30^{\circ} \mathrm{C}$ for one hour and then assayed for activity. We observed that ClpXP incubated at $30{ }^{\circ} \mathrm{C}$ was more active than $\mathrm{ClpXP}$ incubated at $50{ }^{\circ} \mathrm{C}$, suggesting that a partial loss of activity had occurred at high temperature (Figure S4F). However, this assay was performed in the complete absence of other cellular chaperones or substrates and suggests that some ClpXP likely continues to retain activity after exposure to heat stress, while some may become inactivated.

\section{Discussion}

Here, using both a native and an engineered aggregated substrate, we demonstrate that ClpXP has the operational capacity to disassemble and degrade large aggregates that have ClpX degrons. In this study, FtsZ, a native substrate of ClpXP in E. coli, was aggregated in vitro by thermal stress, and we further show that FtsZ also aggregates in vivo when cells are exposed to high temperature (Figure $2 \mathrm{~A}$ and 6A). The observation that FtsZ is aggregation prone is in agreement with a prior study reporting the presence of FtsZ in intracellular aggregates of $\Delta r p o H$ cells incubated at $42{ }^{\circ} \mathrm{C}$ by mass spectrometry (Tomoyasu et al., 2001). FtsZ aggregates are cleared in vitro and in vivo by $\mathrm{ClpXP}$, and $\mathrm{ClpXP}$ does not require the assistance of additional chaperones (Figure 2E, 2F and 6A). Moreover, in the absence of ClpP, ClpX also promotes disassembly of FtsZ and Gfp-ssrA aggregates indicating that disassembly can also occur by a proteolysis-independent mechanism, although disaggregation is 
more efficient in the presence of ClpP. ClpXP-mediated disassembly of Gfp-ssrA aggregates requires ATP in experiments monitoring turbidity (Figure 1E). In addition, the Walker B mutation in ClpX, E185Q, which impairs ATP hydrolysis, also impairs disaggregation of aggGfp-ssrA and, to a lesser extent, aggFtsZ. Aggregate disassembly and resolubilization by $\mathrm{ClpX}$ was previously described using the substrate lambda O protein, and here we show disassembly of aggregates and kinetic monitoring using two additional substrates, as well as reactivation of Gfp-ssrA fluorescence (Wawrzynow et al., 1995). Reactivation of Gfp-ssrA is largely dependent on ATP hydrolysis (Figure 3B), since $\mathrm{ClpX}(\mathrm{E} 185 \mathrm{Q})$ only weakly promotes reactivation of aggregated Gfp-ssrA (Figure 5C), yet ClpX(E185Q) is capable of stable interactions with substrates in the presence of ATP, although they are not unfolded (Hersch et al., 2005;Camberg et al., 2014). It is unlikely that there are soluble, unfolded Gfp-ssrA monomers in solution after heating, since we did not detect them by DLS and it has been demonstrated that soluble, unfolded Gfp rapidly refolds, in 20 to 30 seconds, by a spontaneous reaction that does not require chaperones (Figure 1C) (Makino et al., 1997; Tsien, 1998;Zietkiewicz et al., 2004). Therefore, it is likely that large aggregates contain loosely associated unfolded proteins, which can be removed and reactivated by ClpX and, in the case of Gfp-ssrA, allowed to spontaneously refold. As expected, recognition by $\mathrm{ClpX}$ is highly specific, as Gfp without an ssrA-tag is not reactivated (Figure 4A). 
We also detected partial disaggregation of aggFtsZ by ClpX, but not by $\mathrm{ClpX}(\mathrm{E} 185 \mathrm{Q})$ (Figure 5B). Aggregation of $\mathrm{FtsZ}$ is induced at $65{ }^{\circ} \mathrm{C}$, but the aggregates formed by FtsZ are smaller than those formed by Gfp-ssrA (30 nm and 600 $\mathrm{nm}$, respectively) (Figure 1C and 2D). FtsZ aggregates likely contain 8-10 monomers, based on the average size of a folded FtsZ monomer, which is approximately $40 \AA$ in diameter (Figure 2D) (Oliva et al., 2004). In contrast, Gfp aggregates in this study likely contain more than 120 subunits, based on an average size of a folded Gfp monomer, which is approximately $50 \AA$ across the long axis (van Thor et al., 2005). The small size of the FtsZ aggregate may allow it to be more susceptible to disassembly by ClpX than a larger aggregate.

In the model for disassembly of aggregates by $\mathrm{ClpXP}, \mathrm{ClpX}$ binds to exposed recognition tags on the surface of the aggregate and promotes removal, unfolding and degradation of protomers from within the aggregate (Figure 7A). Removal of protomers eventually leads to destabilization and fragmentation of the aggregate as well as degradation (Figure $1 \mathrm{~F}$ and $2 \mathrm{~F}$ ). Although this process does not require ClpP, it occurs more robustly when ClpP is present than when ClpP is omitted (Figure 1E and 2E). For aggregated substrate reactivation, ClpX likely engages unfolded protomers from the aggregate, which may be internal or loosely bound to the exterior of the aggregate, unfolds and release them. For small aggregates, this activity may be sufficient to lead to fragmentation and capable of promoting reactivation of substrates such as Gfp-ssrA (Figure 7B). 
Finally, we observed large increases in insoluble FtsZ when cells were exposed to two different temperatures, $50{ }^{\circ} \mathrm{C}$, which represents extreme heat shock, and $42{ }^{\circ} \mathrm{C}$, which represents a mild heat shock (Figure 6A, 6B and S4D). At $42{ }^{\circ} \mathrm{C}$, deletion of $c l p B$ was associated with a large accumulation of insoluble FtsZ, suggesting that under mild heat stress, ClpB is the major factor that ensures FtsZ solubility (Figure S4D and S4E). However, we observed a remarkably different result after heat shock at $50{ }^{\circ} \mathrm{C}$ and throughout the recovery period. Specifically, in a $\operatorname{clp} X$ deletion strain, large amounts of insoluble FtsZ accumulate during the recovery period to a greater extent than in a $\operatorname{clpB}$ deletion strain (Figure 6A and 6B). It is unknown if $\mathrm{ClpXP}$ and $\mathrm{ClpB}$ are processing FtsZ aggregates directly in vivo, because we did not observe a reduction of aggregated FtsZ during the recovery period for any strain. FtsZ is typically present at very high levels (5,000 to 20,000 copies per cell) and is essential for cell division in E. coli (Bramhill, 1997). Interestingly, FtsZ also forms linear polymers as part of its normal biological function to promote cell division, and polymers are efficiently recognized, disassembled and degraded by ClpXP (Figure 2F and 2G) (Camberg et al., 2009;Camberg et al., 2014; Viola et al., 2017). Given the diverse conformational plasticity of FtsZ, its use as a model disaggregation and remodeling substrate will be informative for studies of targeting and processing of multisubunit substrates by AAA+ proteins. As with FtsZ, many other ClpXP substrates are detectable in protein aggregates in cells (Flynn et al., 2003;Maisonneuve et al., 2008). Moreover, a previous study showed that ClpXP is important for cell 
viability under thermal stress conditions in cells depleted of DnaK (Tomoyasu et al., 2001). Given that it is estimated that 2-3\% of E. coli proteins are ClpXP substrates, ClpXP likely serves as an additional mechanism to manage accumulation of aggregation-prone proteins in vivo, particularly under extreme stress conditions (Flynn et al., 2003;Maisonneuve et al., 2008).

\section{Funding}

This work was funded by an Institutional Development Award (IDeA) from the National Institute of General Medical Sciences of the National Institutes of Health (\#P20GM103430 to J. Camberg). The funders had no role in study design, data collection and interpretation, or the decision to submit the work for publication.

\section{Acknowledgements}

We thank Sue Wickner, Joel Hoskins, Shannon Doyle, Eric DiBiasio, David Vierra and Katherine Kellenberger for helpful discussions, Paul Johnson and Janet Atoyan for sequencing assistance. Sequencing was performed at the Rhode Island Genomics and Sequencing Center, which is supported in part by the National Science Foundation under EPSCoR Grants Nos. 0554548 \& EPS-1004057.

\section{References}


Abdelhakim, A.H., Oakes, E.C., Sauer, R.T., and Baker, T.A. (2008). Unique contacts direct high-priority recognition of the tetrameric $\mathrm{Mu}$ transposase-DNA complex by the AAA+ unfoldase ClpX. Mol. Cell. 30, 39-50.

Abdelhakim, A.H., Sauer, R.T., and Baker, T.A. (2010). The AAA+ ClpX machine unfolds a keystone subunit to remodel the $\mathrm{Mu}$ transpososome. Proc. Natl. Acad. Sci. USA 107, 2437-2442.

Acebron, S.P., Martin, I., Del Castillo, U., Moro, F., and Muga, A. (2009). DnaKmediated association of $\mathrm{ClpB}$ to protein aggregates. A bichaperone network at the aggregate surface. FEBS Lett. 583, 2991-2996.

Baba, T., Ara, T., Hasegawa, M., Takai, Y., Okumura, Y., Baba, M., Datsenko, K.A., Tomita, M., Wanner, B.L., and Mori, H. (2006). Construction of Escherichia coli K-12 in-frame, single-gene knockout mutants: the Keio collection. Mol. Syst. Biol. 2, 20060008.

Baker, T.A., and Sauer, R.T. (2012). ClpXP, an ATP-powered unfolding and proteindegradation machine. Biochim. Biophys. Acta. 1823, 15-28.

Bolon, D.N., Wah, D.A., Hersch, G.L., Baker, T.A., and Sauer, R.T. (2004). Bivalent tethering of $\mathrm{SspB}$ to $\mathrm{ClpXP}$ is required for efficient substrate delivery: a protein-design study. Mol. Cell. 13, 443-449.

Bramhill, D. (1997). Bacterial cell division. Annu. Rev. Cell Dev. Biol. 13, 395-424.

Bukau, B., and Horwich, A.L. (1998). The Hsp70 and Hsp60 chaperone machines. Cell. 92, 351-366. 
Camberg, J.L., Hoskins, J.R., and Wickner, S. (2009). ClpXP protease degrades the cytoskeletal protein, FtsZ, and modulates FtsZ polymer dynamics. Proc. Natl. Acad. Sci. USA. 106, 10614-10619.

Camberg, J.L., Hoskins, J.R., and Wickner, S. (2011). The interplay of ClpXP with the cell division machinery in Escherichia coli. J. Bacteriol. 193, 1911-1918.

Camberg, J.L., Viola, M.G., Rea, L., Hoskins, J.R., and Wickner, S. (2014). Location of dual sites in E. coli FtsZ important for degradation by ClpXP; one at the Cterminus and one in the disordered linker. PLoS One. 9, e94964.

Conti, J., Viola, M.G., and Camberg, J.L. (2015). The bacterial cell division regulators MinD and MinC form polymers in the presence of nucleotide. FEBS Lett. 589, 201-206.

Datsenko, K.A., and Wanner, B.L. (2000). One-step inactivation of chromosomal genes in Escherichia coli K-12 using PCR products. Proc. Natl. Acad. Sci. USA. 97, 6640-6645.

Davis, J.H., Baker, T.A., and Sauer, R.T. (2009). Engineering synthetic adaptors and substrates for controlled ClpXP degradation. J. Biol. Chem. 284, 21848-21855.

Dougan, D.A., Mogk, A., and Bukau, B. (2002). Protein folding and degradation in bacteria: to degrade or not to degrade? That is the question. Cell. Mol. Life Sci. $59,1607-1616$.

Doyle, S.M., Hoskins, J.R., and Wickner, S. (2007). Inaugural Article: Collaboration between the ClpB AAA+ remodeling protein and the DnaK chaperone system. Proc. Natl. Acad. Sci. USA. 104, 11138-11144. 
Erickson, H.P., Anderson, D.E., and Osawa, M. (2010). FtsZ in bacterial cytokinesis: cytoskeleton and force generator all in one. Microbiol. Mol. Biol. Rev. 74, 504528.

Flynn, J.M., Neher, S.B., Kim, Y.I., Sauer, R.T., and Baker, T.A. (2003). Proteomic discovery of cellular substrates of the ClpXP protease reveals five classes of ClpX-recognition signals. Mol. Cell. 11, 671-683.

Glynn, S.E., Martin, A., Nager, A.R., Baker, T.A., and Sauer, R.T. (2009). Structures of asymmetric $\mathrm{ClpX}$ hexamers reveal nucleotide-dependent motions in a AAA+ protein-unfolding machine. Cell. 139, 744-756.

Gonzalez, J.M., Jimenez, M., Velez, M., Mingorance, J., Andreu, J.M., Vicente, M., and Rivas, G. (2003). Essential cell division protein FtsZ assembles into one monomer-thick ribbons under conditions resembling the crowded intracellular environment. J. Biol. Chem. 278, 37664-37671.

Gottesman, S., Roche, E., Zhou, Y., and Sauer, R.T. (1998). The ClpXP and ClpAP proteases degrade proteins with carboxy-terminal peptide tails added by the SsrA-tagging system. Genes Dev. 12, 1338-1347.

Grimaud, R., Kessel, M., Beuron, F., Steven, A.C., and Maurizi, M.R. (1998). Enzymatic and structural similarities between the Escherichia coli ATPdependent proteases, ClpXP and ClpAP. J. Biol. Chem. 273, 12476-12481.

Gur, E., and Sauer, R.T. (2008). Recognition of misfolded proteins by Lon, a AAA(+) protease. Genes Dev. 22, 2267-2277. 
Hartl, F.U., Bracher, A., and Hayer-Hartl, M. (2011). Molecular chaperones in protein folding and proteostasis. Nature. 475, 324-332.

Hersch, G.L., Burton, R.E., Bolon, D.N., Baker, T.A., and Sauer, R.T. (2005). Asymmetric interactions of ATP with the AAA+ ClpX6 unfoldase: allosteric control of a protein machine. Cell. 121, 1017-1027.

Kain, J., He, G.G., and Losick, R. (2008). Polar localization and compartmentalization of ClpP proteases during growth and sporulation in Bacillus subtilis. J. Bacteriol. 190, 6749-6757.

Kim, Y.I., Burton, R.E., Burton, B.M., Sauer, R.T., and Baker, T.A. (2000). Dynamics of substrate denaturation and translocation by the ClpXP degradation machine. Mol. Cell. 5, 639-648.

Kim, Y.I., Levchenko, I., Fraczkowska, K., Woodruff, R.V., Sauer, R.T., and Baker, T.A. (2001). Molecular determinants of complex formation between Clp/Hsp100 ATPases and the ClpP peptidase. Nat. Struct. Biol. 8, 230-233.

Kirstein, J., Strahl, H., Moliere, N., Hamoen, L.W., and Turgay, K. (2008). Localization of general and regulatory proteolysis in Bacillus subtilis cells. Mol. Microbiol. 70, 682-694.

Kruger, E., Witt, E., Ohlmeier, S., Hanschke, R., and Hecker, M. (2000). The clp proteases of Bacillus subtilis are directly involved in degradation of misfolded proteins. J. Bacteriol. 182, 3259-3265.

Levchenko, I., Seidel, M., Sauer, R.T., and Baker, T.A. (2000). A specificityenhancing factor for the ClpXP degradation machine. Science. 289, 2354-2356. 
Ling, L., Montano, S.P., Sauer, R.T., Rice, P.A., and Baker, T.A. (2015). Deciphering the Roles of Multicomponent Recognition Signals by the AAA+ Unfoldase ClpX. J. Mol. Biol. 427, 2966-2982.

Lu, C., Reedy, M., and Erickson, H.P. (2000). Straight and curved conformations of FtsZ are regulated by GTP hydrolysis. J. Bacteriol. 182, 164-170.

Maisonneuve, E., Fraysse, L., Moinier, D., and Dukan, S. (2008). Existence of abnormal protein aggregates in healthy Escherichia coli cells. J. Bacteriol. 190, 887-893.

Makino, Y., Amada, K., Taguchi, H., and Yoshida, M. (1997). Chaperonin-mediated folding of green fluorescent protein. J. Biol. Chem. 272, 12468-12474.

Martin, A., Baker, T.A., and Sauer, R.T. (2008). Diverse pore loops of the AAA+ ClpX machine mediate unassisted and adaptor-dependent recognition of ssrAtagged substrates. Mol. Cell. 29, 441-450.

Maurizi, M.R., Thompson, M.W., Singh, S.K., and Kim, S.H. (1994). Endopeptidase Clp: ATP-dependent Clp protease from Escherichia coli. Methods Enzymol. 244, 314-331.

Mogk, A., Huber, D., and Bukau, B. (2011). Integrating protein homeostasis strategies in prokaryotes. Cold Spring Harb. Perspect. Biol. 3.

Neher, S.B., Villen, J., Oakes, E.C., Bakalarski, C.E., Sauer, R.T., Gygi, S.P., and Baker, T.A. (2006). Proteomic profiling of ClpXP substrates after DNA damage reveals extensive instability within SOS regulon. Mol. Cell. 22, 193204. 
Oliva, M.A., Cordell, S.C., and Lowe, J. (2004). Structural insights into FtsZ protofilament formation. Nat. Struct. Mol. Biol. 11, 1243-1250.

Park, E.Y., Lee, B.G., Hong, S.B., Kim, H.W., Jeon, H., and Song, H.K. (2007). Structural basis of SspB-tail recognition by the zinc binding domain of ClpX. J. Mol. Biol. 367, 514-526.

Powers, E.T., and Balch, W.E. (2013). Diversity in the origins of proteostasis networks--a driver for protein function in evolution. Nat. Rev. Mol. Cell Biol. $14,237-248$.

Sauer, R.T., and Baker, T.A. (2011). AAA+ proteases: ATP-fueled machines of protein destruction. Annu. Rev. Biochem. 80, 587-612.

Simmons, L.A., Grossman, A.D., and Walker, G.C. (2008). Clp and Lon proteases occupy distinct subcellular positions in Bacillus subtilis. J. Bacteriol. 190, 6758-6768.

Singh, S.K., Grimaud, R., Hoskins, J.R., Wickner, S., and Maurizi, M.R. (2000). Unfolding and internalization of proteins by the ATP-dependent proteases ClpXP and ClpAP. Proc. Natl. Acad. Sci. USA. 97, 8898-8903.

Snider, J., and Houry, W.A. (2008). AAA+ proteins: diversity in function, similarity in structure. Biochem. Soc. Trans. 36, 72-77.

Stephani, K., Weichart, D., and Hengge, R. (2003). Dynamic control of Dps protein levels by ClpXP and ClpAP proteases in Escherichia coli. Mol. Microbiol. 49, 1605-1614. 
Stoecklin, G., and Bukau, B. (2013). Telling right from wrong in life - cellular quality control. Nat. Rev. Mol. Cell Biol. 14, 613-615.

Sweeny, E.A., and Shorter, J. (2016). Mechanistic and Structural Insights into the Prion-Disaggregase Activity of Hsp104. J. Mol. Biol. 428, 1870-1885.

Tomoyasu, T., Mogk, A., Langen, H., Goloubinoff, P., and Bukau, B. (2001). Genetic dissection of the roles of chaperones and proteases in protein folding and degradation in the Escherichia coli cytosol. Mol. Microbiol. 40, 397-413.

Tsien, R.Y. (1998). The green fluorescent protein. Annu. Rev. Biochem. 67, 509-544.

Van Thor, J.J., Georgiev, G.Y., Towrie, M., and Sage, J.T. (2005). Ultrafast and low barrier motions in the photoreactions of the green fluorescent protein. J. Biol. Chem. 280, 33652-33659.

Vera, A., Aris, A., Carrio, M., Gonzalez-Montalban, N., and Villaverde, A. (2005). Lon and ClpP proteases participate in the physiological disintegration of bacterial inclusion bodies. J. Biotechnol. 119, 163-171.

Viola, M.G., Labreck, C.J., Conti, J., and Camberg, J.L. (2017). ProteolysisDependent Remodeling of the Tubulin Homolog FtsZ at the Division Septum in Escherichia coli. PLoS One. 12, e0170505.

Wang, J., Hartling, J.A., and Flanagan, J.M. (1997). The structure of ClpP at 2.3 A resolution suggests a model for ATP- dependent proteolysis. Cell. 91, 447-456.

Wawrzynow, A., Wojtkowiak, D., Marszalek, J., Banecki, B., Jonsen, M., Graves, B., Georgopoulos, C., and Zylicz, M. (1995). The ClpX heat-shock protein of Escherichia coli, the ATP-dependent substrate specificity component of the 
ClpP-ClpX protease, is a novel molecular chaperone. EMBO J.. 14, 18671877.

Wickner, S., Maurizi, M.R., and Gottesman, S. (1999). Posttranslational quality control: folding, refolding, and degrading proteins. Science. 286, 1888-1893.

Winkler, J., Seybert, A., Konig, L., Pruggnaller, S., Haselmann, U., Sourjik, V., Weiss, M., Frangakis, A.S., Mogk, A., and Bukau, B. (2010). Quantitative and spatio-temporal features of protein aggregation in Escherichia coli and consequences on protein quality control and cellular ageing. EMBO J 29, 910923.

Yakhnin, A.V., Vinokurov, L.M., Surin, A.K., and Alakhov, Y.B. (1998). Green fluorescent protein purification by organic extraction. Protein Expr. Purif. 14, $382-386$.

Zietkiewicz, S., Krzewska, J., and Liberek, K. (2004). Successive and synergistic action of the Hsp70 and Hsp100 chaperones in protein disaggregation. J. Biol. Chem. 279, 44376-44383.

Zietkiewicz, S., Lewandowska, A., Stocki, P., and Liberek, K. (2006). Hsp70 chaperone machine remodels protein aggregates at the initial step of Hsp70Hsp100-dependent disaggregation. J. Biol. Chem. 281, 7022-7029.

Zolkiewski, M. (1999). ClpB cooperates with DnaK, DnaJ, and GrpE in suppressing protein aggregation. A novel multi-chaperone system from Escherichia coli. J. Biol. Chem. 274, 28083-28086. 
Zolkiewski, M. (2006). A camel passes through the eye of a needle: protein unfolding activity of Clp ATPases. Mol. Microbiol. 61, 1094-1100. 
TABLE 1. E. coli strains and plasmids used in this study

\begin{tabular}{|c|c|c|}
\hline $\begin{array}{l}\text { Strain or } \\
\text { Plasmid }\end{array}$ & Genotype & $\begin{array}{l}\text { Source, reference or } \\
\text { Construction }\end{array}$ \\
\hline \multicolumn{3}{|l|}{ Strains } \\
\hline BW25113 & $\begin{array}{l}\mathrm{F}, \mathrm{DE}(\operatorname{araD}-a r a B) 567, \text { lacZ4787(del)(::rrnB-3), } \\
L A M-, r p h-1, \mathrm{DE}(r h a D-r h a B) 568, h s d R 514\end{array}$ & Datsenko and Wanner, 2000 \\
\hline JW0429 & 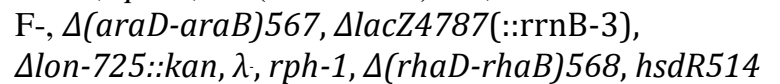 & Baba et al., 2006 \\
\hline JW0428 & $\begin{array}{l}\mathrm{F}-, \Delta(\operatorname{araD}-a r a B) 567, \Delta l a c Z 4787(:: \mathrm{rrnB}-3) \\
\Delta c l p X 724:: k a n, \lambda, \text { rph-1, } \Delta(\text { rhaD-rhaB }) 568, \text { hsdR514 }\end{array}$ & Baba et al., 2006 \\
\hline JW0427 & $\begin{array}{l}\text { F-, } \Delta(\text { araD-araB }) 567, \Delta l a c Z 4787(: \because \text { rrnB-3), } \\
\Delta c l p P 723:: k a n, \lambda, \text { rph-1, } \Delta(\text { rhaD-rhaB }) 568, \text { hsdR514 }\end{array}$ & Baba et al., 2006 \\
\hline JW2573 & $\begin{array}{l}\mathrm{F}-, \Delta(\text { araD-araB }) 567, \Delta l a c Z 4787(:: \mathrm{rrnB}-3) \\
\Delta \operatorname{clpB} 757:: k a n, \lambda, \text { rph-1, } \Delta(\text { rhaD-rhaB }) 568, \text { hsdR514 }\end{array}$ & Baba et al., 2006 \\
\hline JW0866 & $\begin{array}{l}\mathrm{F}-, \Delta(\operatorname{araD}-a r a B) 567, \Delta l a c Z 4787(:: \mathrm{rrnB}-3) \\
\text { AclpA783::kan, } \lambda, \text { rph-1, } \Delta(\text { rhaD-rhaB }) 568, \text { hsdR514 }\end{array}$ & Baba et al., 2006 \\
\hline JW3902 & $\begin{array}{l}\mathrm{F}-, \Delta(\operatorname{araD}-a r a B) 567, \text { LlacZ4787(::rrnB-3), } \\
\text { AhslU790::kan, } \lambda, \text { rph-1, } \Delta(\text { rhaD-rhaB }) 568, \text { hsdR514 }\end{array}$ & Baba et al., 2006 \\
\hline JW3903 & $\begin{array}{l}\mathrm{F}-, \Delta(\operatorname{araD}-a r a B) 567, \Delta l a c Z 4787(:: \mathrm{rrnB}-3) \\
\Delta h s l V 720: \because k a n, \lambda, r p h-1, \Delta(\text { rhaD-rhaB }) 568, \text { hsdR514 }\end{array}$ & Baba et al., 2006 \\
\hline JW0013 & $\begin{array}{l}\mathrm{F}-, \Delta(\operatorname{araD}-a r a B) 567, \Delta l a c Z 4787(:: \mathrm{rrnB}-3) \\
\Delta d n a K 734:: k a n, \lambda, \text { rph-1, } \Delta(\text { rhaD-rhaB }) 568, \text { hsdR514 }\end{array}$ & Baba et al., 2006 \\
\hline JW0462 & $\begin{array}{l}\mathrm{F}-, \Delta(\operatorname{araD}-a r a B) 567, \Delta l a c Z 4787(:: \mathrm{rrnB}-3) \\
\Delta h t p G 757:: k a n, \lambda, \text { rph-1, } \Delta(\text { rhaD-rhaB }) 568, h s d R 514\end{array}$ & Baba et al., 2006 \\
\hline JC0259 & MG1655 $4 c l p X:: k a n$ & Camberg et al., 2011 \\
\hline \multicolumn{3}{|l|}{ Plasmids } \\
\hline pET-ClpX & kan & Camberg et al. 2009 \\
\hline pET-ClpP & kan & Maurizi et al. 1994 \\
\hline pET-FtsZ & kan & Camberg et al. 2009 \\
\hline pET-FtsZ $(\Delta \mathrm{C} 67)$ & kan & This study \\
\hline pET-H - $_{6}$ Gfp(uv) & kan & This study \\
\hline pBad-Gfp-ssrA & amp & Singh et al., 2000 \\
\hline pClpX(E185Q) & amp & Camberg et al., 2011 \\
\hline
\end{tabular}




\section{Figure 1 - Disaggregation and degradation of aggregated Gfp-ssrA by ClpXP.}

(A) The fluorescence emission spectra $(450-600 \mathrm{~nm})$ of Gfp-ssrA $(1.0 \mu \mathrm{M})$ (green) and heat-treated Gfp-ssrA $(1.0 \mu \mathrm{M})$ (black) $\left(85^{\circ} \mathrm{C}\right.$ for 15 minutes) were measured using an excitation wavelength of $395 \mathrm{~nm}$. Plotted curves are representative of three traces.

(B) DLS was performed for Gfp-ssrA $(1.0 \mu \mathrm{M})$ (green) as described to determine particle size (nm) distribution.

(C) DLS was performed for heat-treated Gfp-ssrA (aggGfp-ssrA) $(1.0 \mu \mathrm{M})$ (black) as described to determine particle size (nm) distribution.

(D) Aggregation by $90^{\circ}$-angle light scatter was measured for Gfp-ssrA (1.5 $\left.\mu \mathrm{M}\right)$ (open circles) in a cuvette attached to a circulating water bath held at $80^{\circ} \mathrm{C}$. Light scattering was monitored for 15 minutes.

(E) Disaggregation of aggGfp-ssrA $(1 \mu \mathrm{M})$ was monitored by $90^{\circ}$-angle light scatter as described in Materials and Methods. Disaggregation reactions contained aggGfpssrA $(1 \mu \mathrm{M})$ (black circles), ClpX $(0.5 \mu \mathrm{M})$ and ATP (blue circles), ClpX $(0.5 \mu \mathrm{M})$ and ClpP $(0.6 \mu \mathrm{M})$ (gold circles), ClpX $(0.5 \mu \mathrm{M}), \mathrm{ClpP}(0.6 \mu \mathrm{M})$, and ATP $(4 \mathrm{mM})$ (red circles), and a regenerating system, where indicated. Light scattering was monitored for 120 minutes. Curves shown are representative of at least three replicates.

(F) Degradation of Gfp-ssrA and aggGfp-ssrA was monitored as described in Materials and Methods in reactions containing Gfp-ssrA $(1 \mu \mathrm{M})$ or aggGfp-ssrA (1 
$\mu \mathrm{M})$, where indicated, and $\operatorname{ClpX}(0.5 \mu \mathrm{M}), \mathrm{ClpP}(0.6 \mu \mathrm{M}), \mathrm{ATP}(4 \mathrm{mM})$, and a regenerating system, where indicated. Reactions were incubated at $23{ }^{\circ} \mathrm{C}$ for 120 minutes and samples were analyzed by SDS-PAGE and Coomassie stain. 

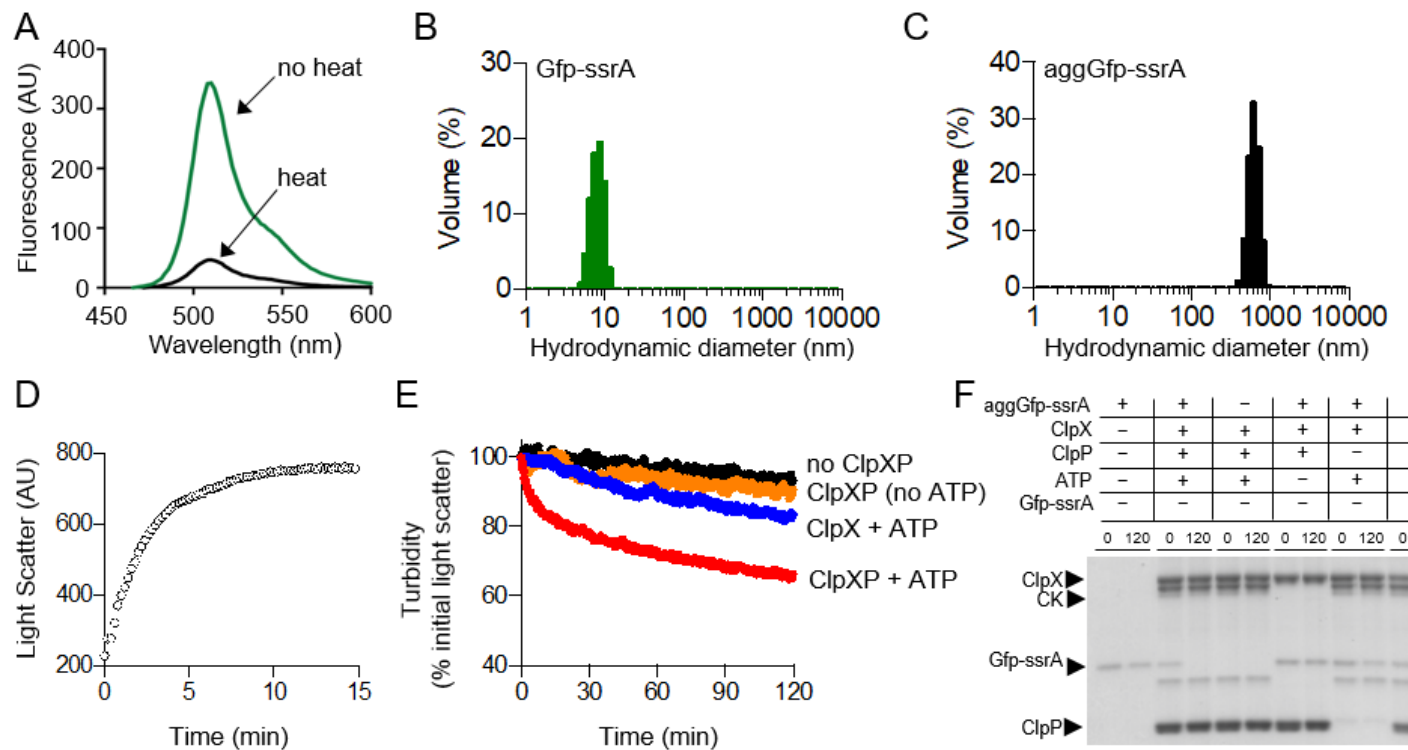

E
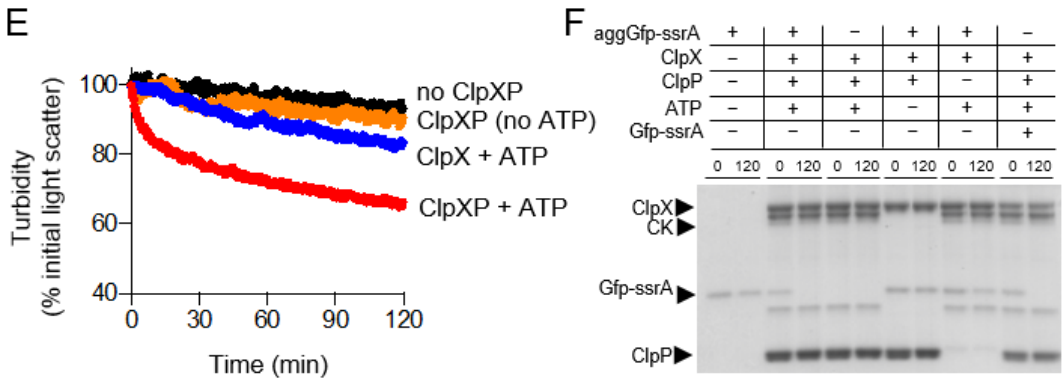
Figure 2 - Aggregation and disaggregation of native ClpXP substrate FtsZ.

(A) Aggregation by $90^{\circ}$-angle light scatter was measured for FtsZ ( $\left.5 \mu \mathrm{M}\right)$ (black circles) in a cuvette attached to a circulating water bath at $65^{\circ} \mathrm{C}$ for 30 minutes as described in Materials and Methods. The curve shown is representative of at least three replicates.

(B) Rates of GTP hydrolysis were measured for FtsZ (5 $\mu \mathrm{M})$ (gray) and aggFtsZ (5 $\mu \mathrm{M})$ (black) with GTP $(1 \mathrm{mM})$ for 15 minutes at $30{ }^{\circ} \mathrm{C}$, as described in Materials and Methods. The average rate was determined from at least four replicates.

(C) DLS was performed for FtsZ $(5 \mu \mathrm{M})$ (gray) as described to determine particle size (nm) distribution.

(D) DLS was performed for aggFtsZ $(5 \mu \mathrm{M})$ (black) as described to determine particle size $(\mathrm{nm})$ distribution.

(E) Disaggregation of aggFtsZ $(5 \mu \mathrm{M})$ was monitored by $90^{\circ}$-angle light scatter as described in Materials and Methods. Disaggregation reactions contained aggFtsZ (5 $\mu \mathrm{M})$ (black circles) or aggFtsZ $(5 \mu \mathrm{M})$ and ClpX $(1 \mu \mathrm{M})$ (blue circles), or aggFtsZ (5 $\mu \mathrm{M}), \operatorname{ClpX}(1 \mu \mathrm{M})$, and $\operatorname{ClpP}(1 \mu \mathrm{M})$ (red circles), with ATP $(4 \mathrm{mM})$ and a regenerating system. Light scattering was monitored for 120 minutes. The curves shown are representative of at least three replicates.

(F) Degradation was monitored for FtsZ and aggFtsZ as described in Materials and Methods in reactions containing FtsZ $(6 \mu \mathrm{M})$, aggFtsZ $(6 \mu \mathrm{M}), \mathrm{ClpX}(0.5 \mu \mathrm{M}), \mathrm{ClpP}$ $(0.5 \mu \mathrm{M})$, ATP $(4 \mathrm{mM})$ and a regenerating system, where indicated. For degradation 
of FtsZ, GMPCPP $(0.5 \mathrm{mM})$ was included to promote the assembly of stable polymers. Degradation reactions were incubated at $23{ }^{\circ} \mathrm{C}$ for 120 minutes. To detect protein loss due to degradation, samples from 0 and 120 minutes were analyzed by SDS-PAGE to solubilize any remaining aggregates.

(G) Degradation was monitored for FL-FtsZ (125 pmol) incubated in the presence of GMPCPP (0.5 mM) for 3 minutes, then ATP (4 mM), a regenerating system, and increasing concentrations of $\operatorname{ClpXP}(0,0.25,0.5$ and $1 \mu \mathrm{M}$ as shown $)$ were added and reactions were incubated for an additional 30 minutes at $23{ }^{\circ} \mathrm{C}$. Reactions were centrifuged at $129,000 \times \mathrm{x}$ for 30 minutes at $23{ }^{\circ} \mathrm{C}$. Pellet-associated FtsZ was quantified by fluorescence, and each data point is an average of at least three replicates. 


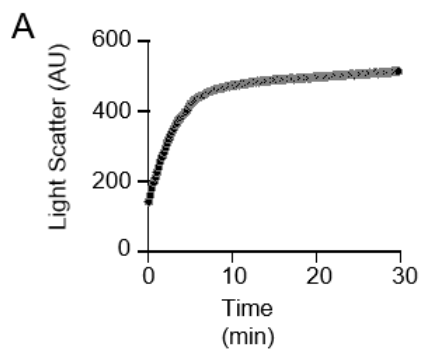

E

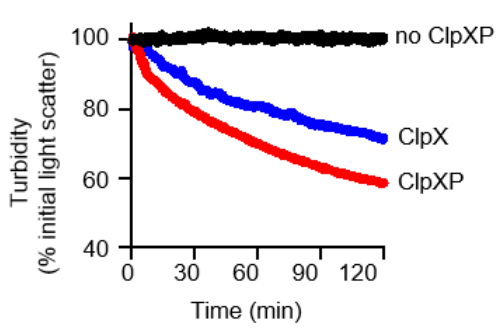

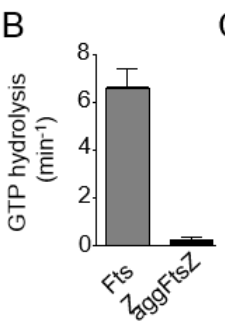
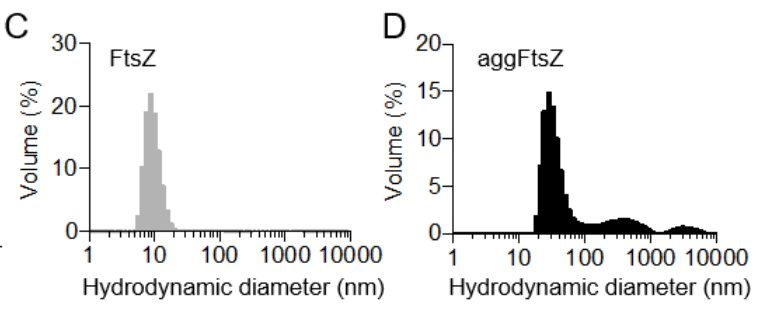

$\mathrm{F}$

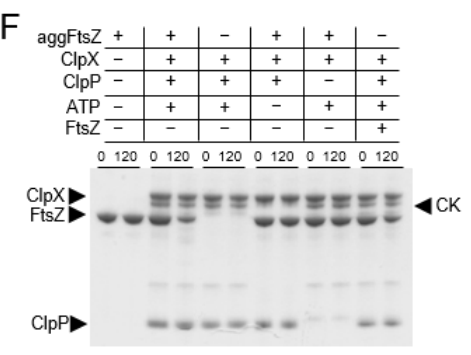

G

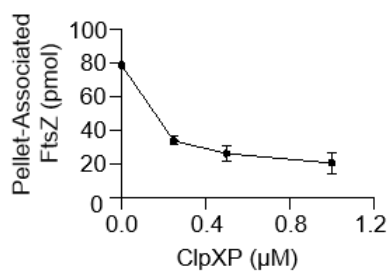




\section{Figure 3 - Reactivation of aggregated Gfp-ssrA in the presence of ClpX.}

(A) Reactivation of aggGfp-ssrA $(1.0 \mu \mathrm{M})$ was monitored as described in Materials and Methods in the absence (black circles) and presence (blue circles) of ClpX (0.3 $\mu \mathrm{M})$, ATP $(4 \mathrm{mM})$, and a regenerating system. Fluorescence emission (AU) was monitored for 30 minutes. The curves shown are representative of at least three replicates.

(B) Reactivation of aggGfp-ssrA $(1.0 \mu \mathrm{M})$ was monitored in the absence (black circles) or presence of $\mathrm{ClpX}(0.3 \mu \mathrm{M})$, ATP $(4 \mathrm{mM})$ and a regenerating system (blue circles), ATP $\gamma \mathrm{S}(2 \mathrm{mM})$ (orange circles), ADP $(2 \mathrm{mM})$ (green circles), or no nucleotide (gray circles), where indicated. Fluorescence emission (AU) was monitored for 60 minutes. The curves shown are representative of at least three replicates. 

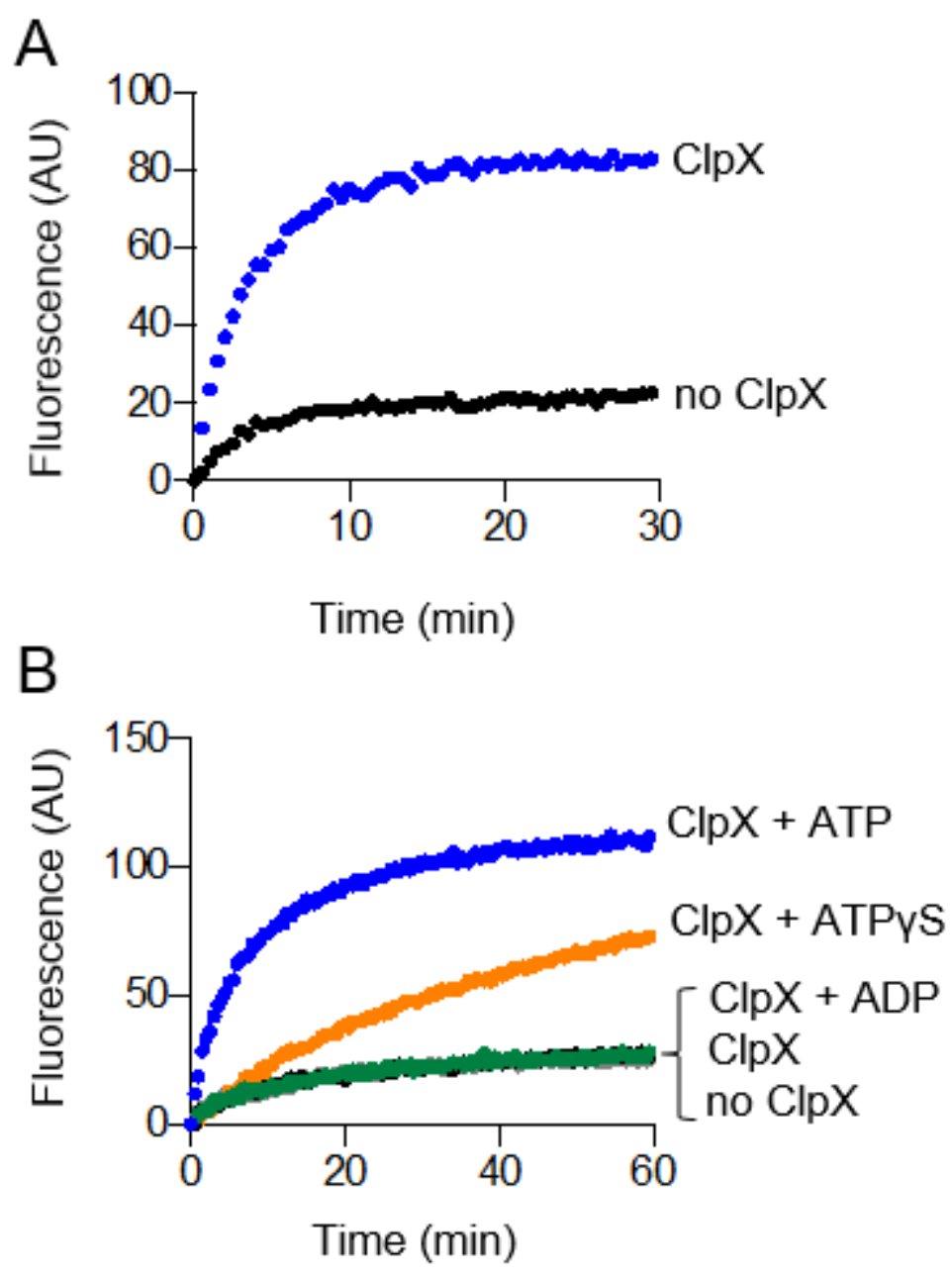
Figure 4 - Aggregation and disaggregation of ClpXP substrates with and without recognition motifs.

(A) Reactivation of aggGfp-ssrA $(1.0 \mu \mathrm{M})$ alone (dark gray circles) or in the presence of $\operatorname{ClpX}(0.3 \mu \mathrm{M})$ (blue circles), and reactivation of aggGfp(uv) alone (1.0 $\mu \mathrm{M})$ (light gray circles) or in the presence of $\operatorname{ClpX}(0.3 \mu \mathrm{M})$ (green circles), where indicated, was monitored with ATP (4 mM) and a regenerating system as described in Materials and Methods. Fluorescence emission (AU) was monitored for 60 minutes. The plotted curves are representative of at least three replicates.

(B) Rates of GTP hydrolysis were determined as described in Materials and Methods for $\operatorname{FtsZ}(\Delta \mathrm{C} 67)(5 \mu \mathrm{M})$ (gray) and $\operatorname{aggFtsZ}(\Delta \mathrm{C} 67)(5 \mu \mathrm{M})$ (black), where indicated, incubated with GTP $(1 \mathrm{mM})$ for 15 minutes at $30{ }^{\circ} \mathrm{C}$. The average rate was determined from at least four replicates.

(C) Disaggregation was monitored by $90^{\circ}$-angle light scatter for aggFtsZ( $\left.\triangle \mathrm{C} 67\right)(5$ $\mu \mathrm{M})$ alone (black), aggFtsZ( $\Delta \mathrm{C} 67)(5 \mu \mathrm{M})$ in the presence of ClpXP $(0.5 \mu \mathrm{M})$, ATP $(4 \mathrm{mM})$ and a regenerating system (red), or aggFtsZ $(5 \mu \mathrm{M})$ in the presence of ClpXP $(0.5 \mu \mathrm{M})$, ATP $(4 \mathrm{mM})$ and a regenerating system (blue) where indicated as described in Materials and Methods. Light scattering was monitored for 120 minutes. The curves shown are representative of at least three replicates. 

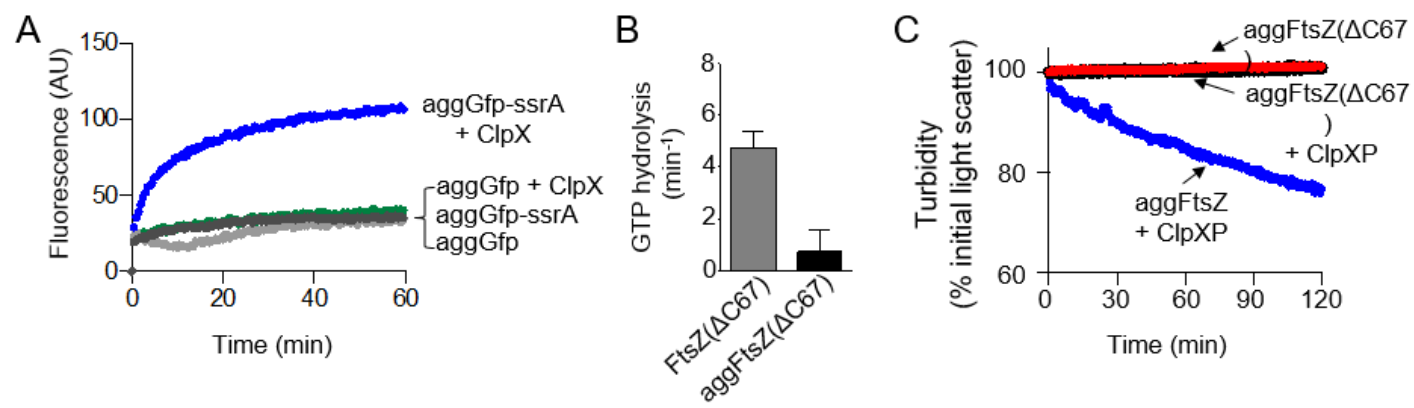
Figure 5 - Disaggregation and reactivation of ClpX substrates in the presence of $\operatorname{ClpX(E185Q).~}$

(A) Disaggregation was monitored by $90^{\circ}$-angle light scatter, as described in Materials and Methods for aggGfp-ssrA $(1.0 \mu \mathrm{M})$ alone (black circles) or in the presence of ClpX $(0.5 \mu \mathrm{M})$ (blue circles) or ClpX (E185Q) (0.5 $\mu \mathrm{M})$ (open circles), where indicated, with ATP (4 mM), and a regenerating system. Light scattering was monitored for 120 minutes. The curves shown are representative of at least three replicates.

(B) Disaggregation was monitored by $90^{\circ}$-angle light scatter for aggFtsZ $(5 \mu \mathrm{M})$, ClpX $(0.5 \mu \mathrm{M})$ or $\mathrm{ClpX}(\mathrm{E} 185 \mathrm{Q})(0.5 \mu \mathrm{M})$ where indicated, ATP $(4 \mathrm{mM})$, and a regenerating system for 120 minutes as described in Materials and Methods. The curves shown are representative of at least three replicates.

(C) Reactivation was monitored as described in Materials and Methods for aggGfpssrA $(1.0 \mu \mathrm{M})$ alone (black circles) or in the presence of $\mathrm{ClpX}(0.3 \mu \mathrm{M})$ (blue circles) or $\mathrm{ClpX}(\mathrm{E} 185 \mathrm{Q})(0.3 \mu \mathrm{M})$ (open circles), with ATP $(4 \mathrm{mM})$ and a regenerating system, where indicated. Fluorescence emission (AU) was monitored for 90 minutes. The curves shown are representative of at least three replicates. 

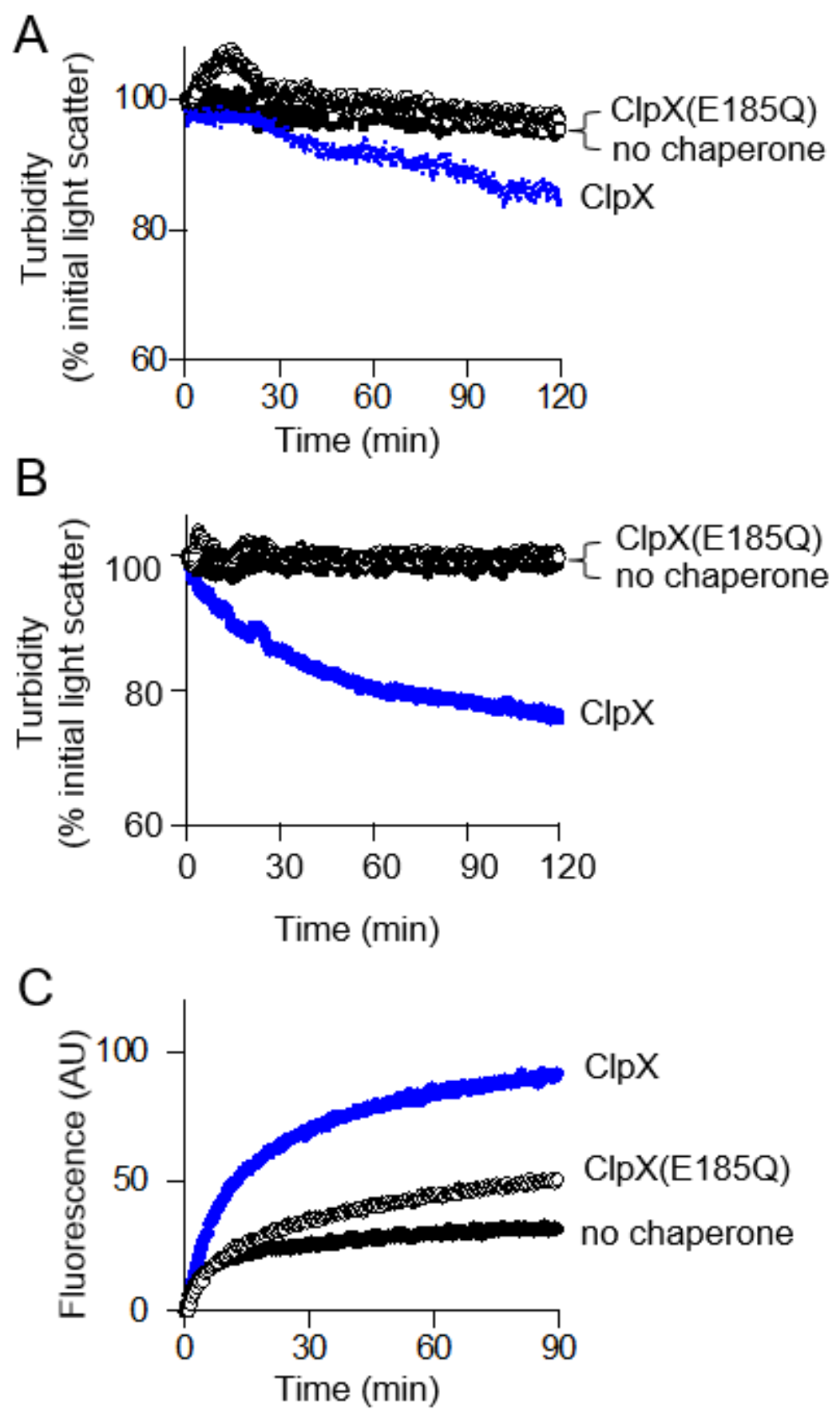
Figure 6 - FtsZ aggregation in deletion strains after heat shock.

(A) FtsZ levels were compared in insoluble cell extracts prepared from single gene deletion strains (Table 1) after heat shock at $50{ }^{\circ} \mathrm{C}$ for 1 hour and recovery $\left(30^{\circ} \mathrm{C}\right)$ as described in Materials and Methods. Cells were collected and insoluble protein extracts were analyzed by immunoblotting using anti-FtsZ antibodies. Relative FtsZ levels were quantified by densitometry from four independent experiments. Where indicated, '*' represents a $p$-value of 0.03 .

(B) Insoluble FtsZ levels were monitored during the $30{ }^{\circ} \mathrm{C}$ recovery period $(0,20$ and 40 minutes) after heat shock at $50{ }^{\circ} \mathrm{C}$ for 60 minutes in wild type, $\Delta c l p B$ and $\Delta c l p X$ deletion strains. 


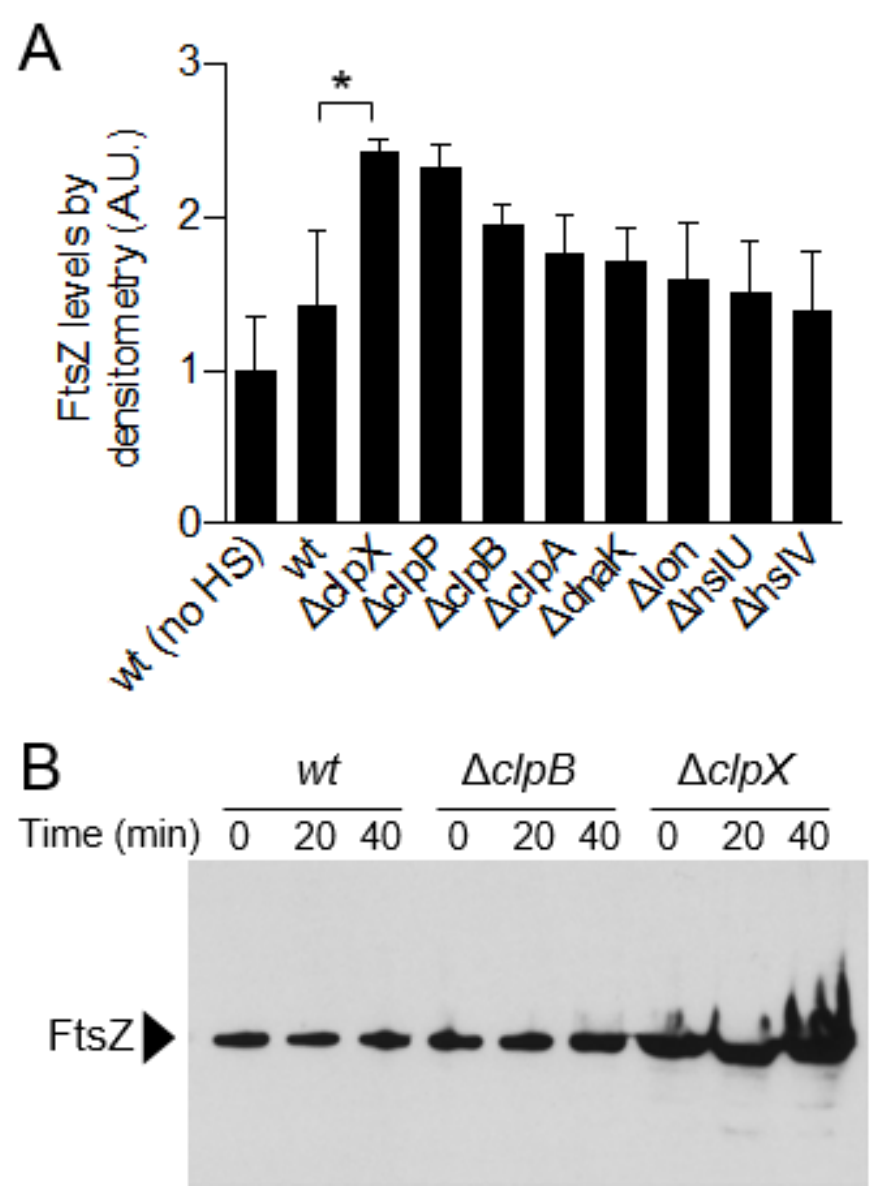




\section{Figure 7 - Model of aggregate disassembly.}

(A) ClpXP binds to aggregated substrates bearing a ClpX-recognition motif. ClpXP unfolds and degrades protomers from within the aggregate, leading to fragmentation and disassembly in an ATP-dependent manner.

(B) ClpX binds to aggregates that contain unfolded proteins bearing a ClpXrecognition motif. Unfolded proteins loosely associated with the aggregate surface are reactivated by $\mathrm{ClpX}$ through a direct protein interaction that requires $\mathrm{ATP}$-dependent unfolding. 

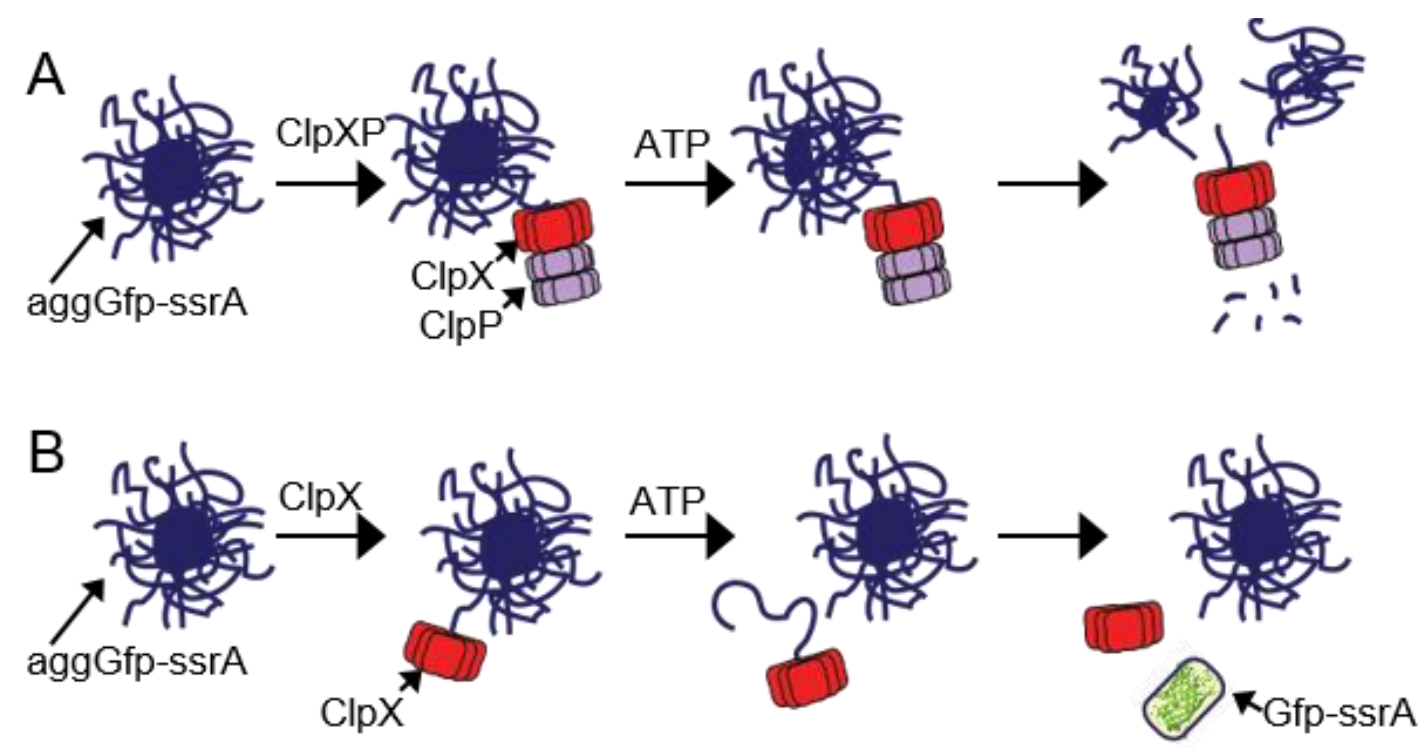


\section{Supplemental Figure S1 - Heat-aggregation of Gfp-ssrA.}

The fluorescence emission of aggGfp-ssrA $(1.0 \mu \mathrm{M})$ (black circles) was monitored as described in Materials and Methods for 90 minutes. 


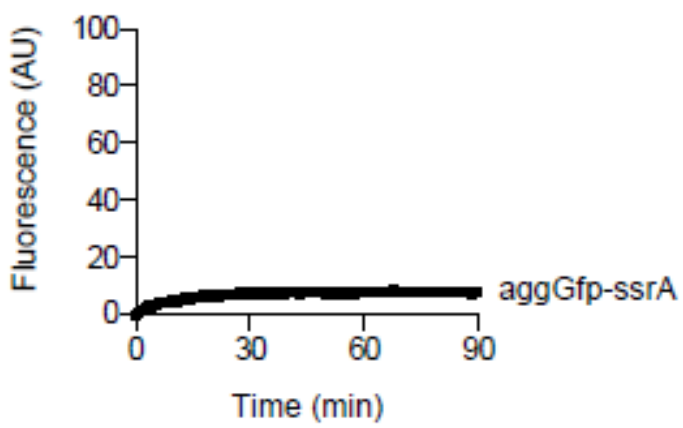


Supplemental Figure S2 - Unfolding and degradation of aggregated Gfp-ssrA by ClpXP.

Unfolding and degradation were monitored for aggGfp-ssrA $(1.0 \mu \mathrm{M})$ alone (black circles) or in the presence of ClpP $(0.3 \mu \mathrm{M})$ (gold circles), $\mathrm{ClpX}(0.3 \mu \mathrm{M})$ and $\mathrm{ClpP}(0$. $\mu \mathrm{M})$ (red circles) with ATP $(4 \mathrm{mM})$, where indicated. Fluorescence emission (AU) was monitored as described in Materials and Methods. 


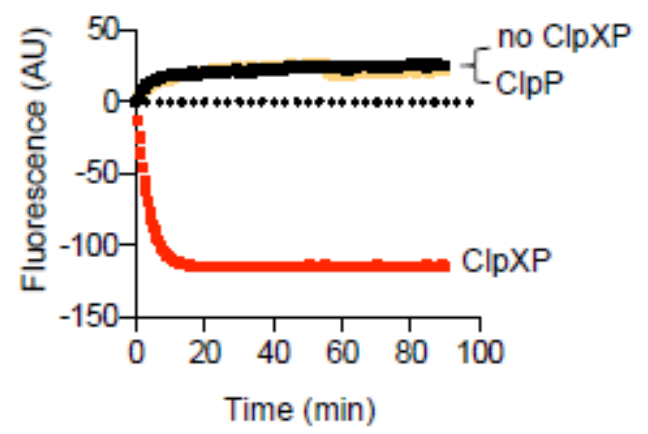




\section{Supplemental Figure S3 - Degradation of FtsZ and FtsZ( $\Delta$ C67) by ClpXP.}

Degradation was monitored for FtsZ $(6 \mu \mathrm{M})$ and FtsZ( $\Delta$ C67), ClpXP $(0.5 \mu \mathrm{M})$, ATP (4 mM), GMPCPP $(0.5 \mathrm{mM})$, and a regenerating system where indicated at $23{ }^{\circ} \mathrm{C}$ for 120 minutes as described in Materials and Methods, and samples were analyzed by SDS-PAGE and Coomassie stain. 


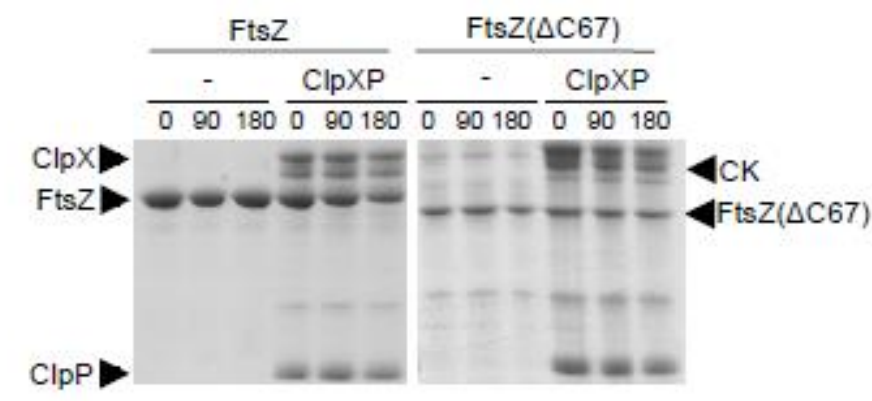




\section{Supplemental Figure S4 - Insoluble FtsZ in deletion strains after heat-treatment.}

(A) Single gene deletion strains (Table 1) were incubated at $50{ }^{\circ} \mathrm{C}$ for 1 hour and recovered as described in Materials and Methods. Cells from deletion strains were collected and insoluble protein extracts were collected as described and analyzed by reducing SDS-PAGE. Immunoblots were performed with antibodies to FtsZ or ClpX as described.

(B) Total protein present in insoluble cell extracts shown in (A) after heat shock at 50 ${ }^{\circ} \mathrm{C}$ and recovery was detected by transferring proteins to a nitrocellulose membrane and staining with Ponceau.

(C) Cell viability for all strains in (A) was determined by measuring colony forming units (CFU ml ${ }^{-1}$ ) of cultures before heating ('pre-HS'), after heat treatment at $50{ }^{\circ} \mathrm{C}$ for 1 hour ('post-HS'), and after 35 minutes of recovery at $30{ }^{\circ} \mathrm{C}$ ('post-rec').

(D) FtsZ levels were compared in single gene deletion strains after heat shock at $42{ }^{\circ} \mathrm{C}$ for 30 minutes and recovery $\left(30{ }^{\circ} \mathrm{C}\right)$ as described in Materials and Methods. Cells were collected and insoluble protein extracts were analyzed by immunoblotting with antibodies to FtsZ as described.

(E) Insoluble FtsZ levels were monitored in wild type, $\Delta c l p X$ and $\Delta c l p B$ deletion strains before heat shock $\left(50{ }^{\circ} \mathrm{C}\right.$ for one hour or $42{ }^{\circ} \mathrm{C}$ for 30 minutes, where indicated) and during the $30{ }^{\circ} \mathrm{C}$ recovery period (0,20 and 40 minutes). At the indicated times, cells were collected from cultures and insoluble protein extracts were analyzed by immunoblotting with antibodies to FtsZ as described. 
(F) Thermal stability of ClpXP was assayed by incubation of ClpX $(0.5 \mu \mathrm{M})$ and $\mathrm{ClpP}$ $(0.7 \mu \mathrm{M})$ in phosphate buffered saline supplemented with ATP $(4 \mathrm{mM}) \mathrm{MgCl}_{2}(10$ $\mathrm{mM})$, glycerol (15\%), Triton X-100 (0.005\%), and TCEP (1 mM). Reactions containing ClpXP were added to a preheated quartz cuvette attached to a circulating water bath set to $50{ }^{\circ} \mathrm{C}$ or $30{ }^{\circ} \mathrm{C}$, where indicated, and incubated for one hour. The circulating water bath was rapidly cooled to $30{ }^{\circ} \mathrm{C}$, the reactions were supplemented with ATP and regenerating system, Gfp-ssrA $(0.2 \mu \mathrm{M})$ was added, and fluorescence was monitored with time in the absence (black) or presence of ClpXP, treated at $50{ }^{\circ} \mathrm{C}$ (red) or $30^{\circ} \mathrm{C}$ (aqua). 


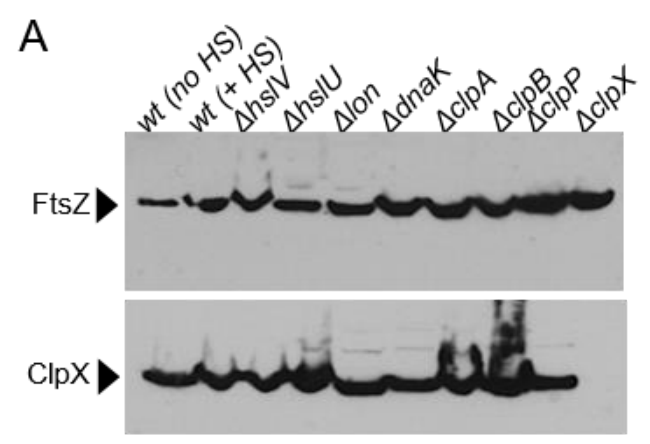

B

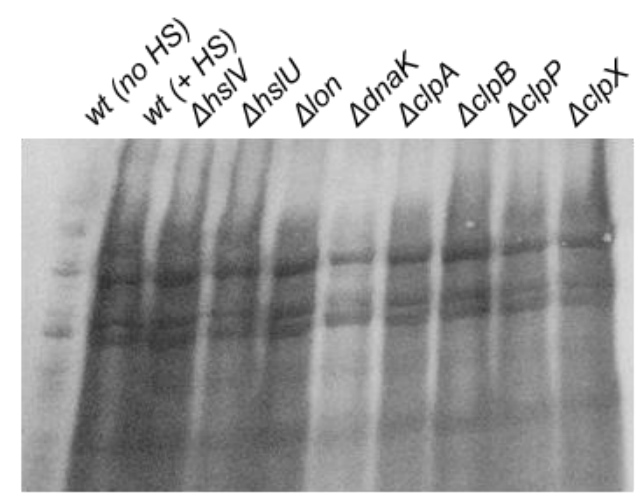

C

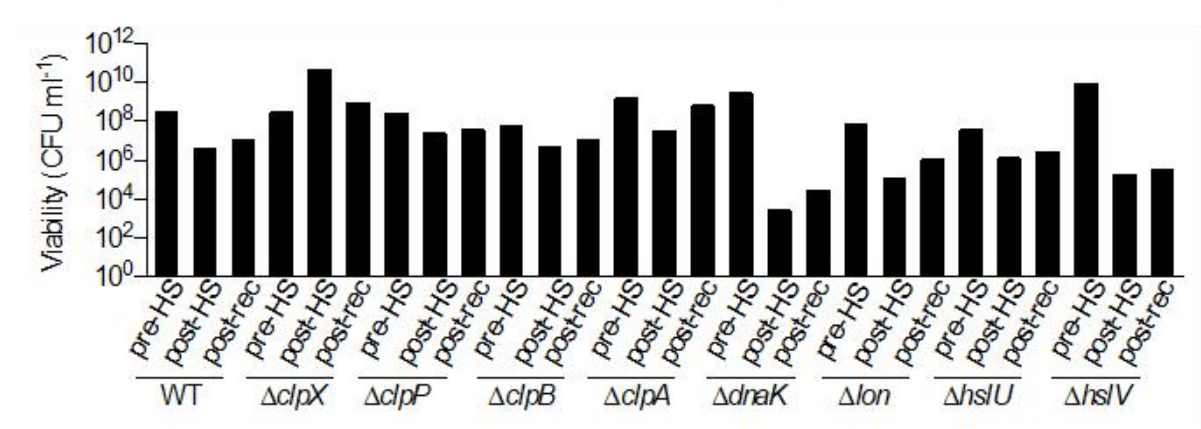

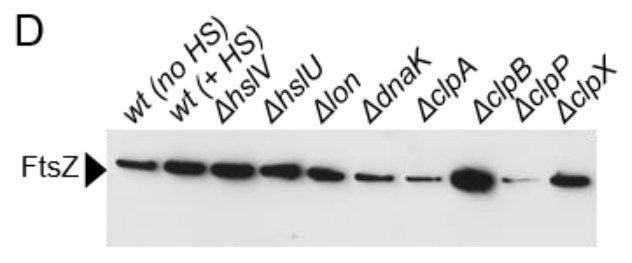
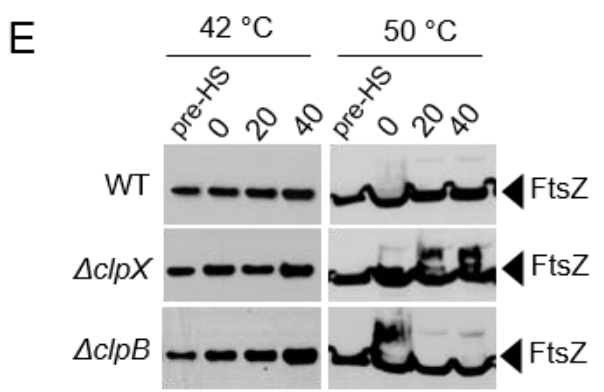

F

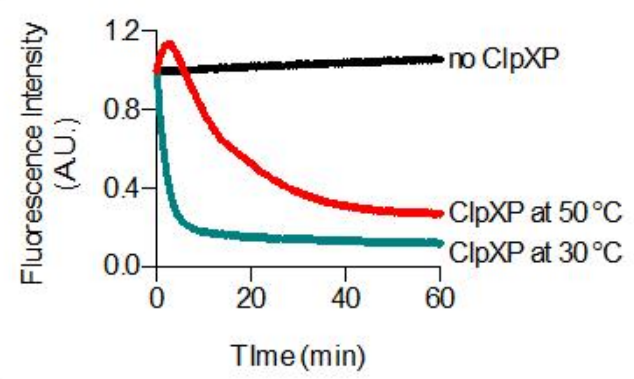

